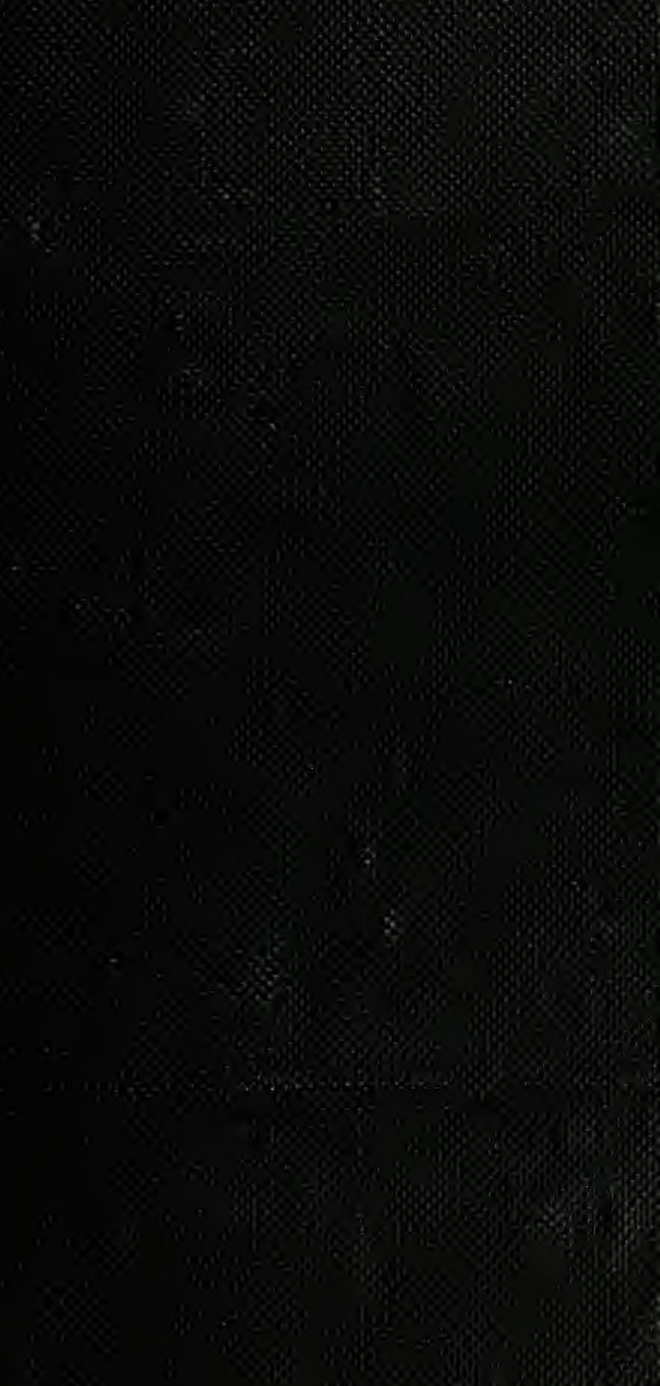




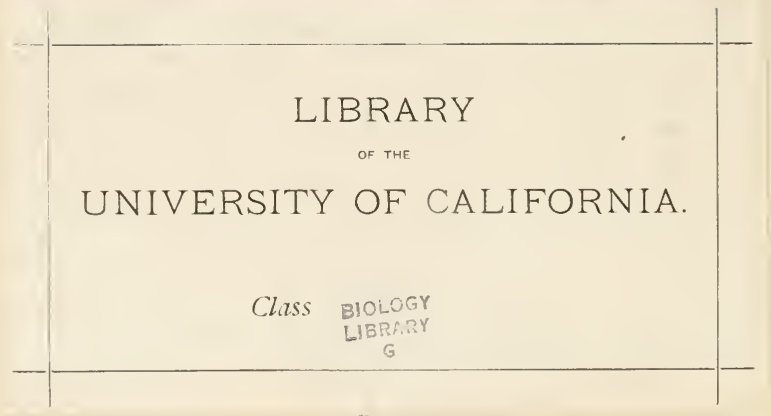

EFNERA: 
(5) 


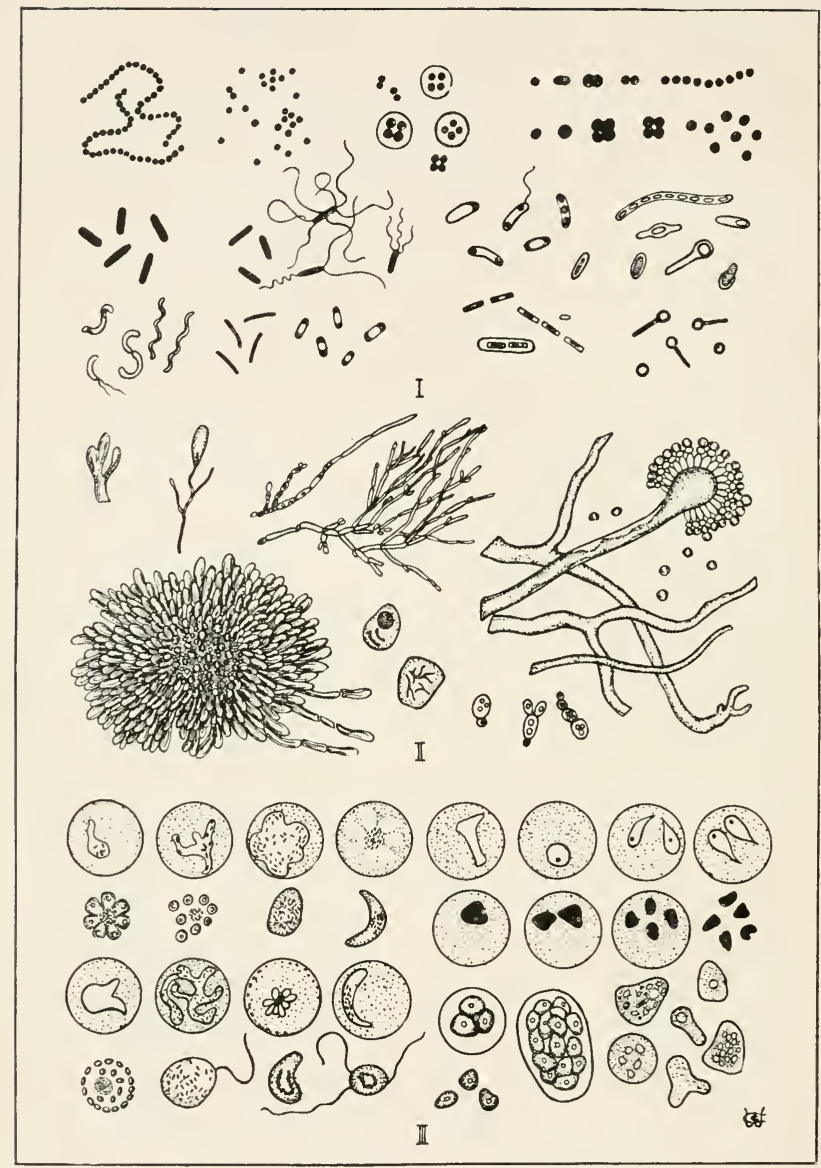

I. Bacteria.

II. Fungi.

III. Protozoa.

CAUSES OF INFECTIOUS DISEASES. 


Digitized by the Internet Archive in 2008 with funding from Microsoft Corporation 



\title{
THE PATHOLOGY
}

\author{
A ND \\ DIFFERENTIAL DIAGNOSIS \\ $\mathrm{OF}$
}

INFEC'TIOUS DISEASES OF ANIMALS

By VERANUS ALVA MOORE, B.S., M.D.

Professor of Comparative Pathology, Bacteriology and Meat Inspection, New York state Veterinary College, Cornell Lniversity, Ithaca, N. Y.

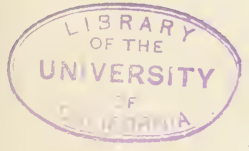

DANIEL ELMER SALMON, D.V.M.

Chief of the Bureau of Animal Industry, Lnited states Department of Agriculture.

ILLUSTRATED.

ITHACA, N. Y, :

TAYLOR \& CARPENTER.

1002. 
COPYRIGHT, I902. Bi Veranus Alva Moore:

GENERAL

PRESS OF

JOURNAL JOB DEPARTMENT

ITHACA, N. $Y$ 
TO DANIEL ELMER SALMON

LARGELY THROUCH WHOSE LABORS THERE WAS ESTABLISHED IN THE UNITED STATES DEPARTMENT OF AGRICULTURE THE BUREAU OF ANIMAL INDUSTRY WHICH HAS MADE POSSIBLE EXTENSIVE INVESTIGATIONS INTO THE NATURE OF EPIZOÖTIC DISEASES IN AMERICA AND WHO FOR EIGHTEEN YEARS HAS DIRECTED THESE INVESTIGATIONS

THIS VOLUME IS DEDICATED. 



\section{PREFACE.}

The literature on infection and the etiology and morbid anatomy of infectious diseases of animals is exceedingly rich in the results of new discoveries and important investigations. However, students just beginning this study and following a prescribed curriculum have not the time nor are they prepared to read with profit the detailed records of original research. Such publications seem to be better adapted for those doing advanced or graduate work. Furthermore, many of these publications are out of print and are only available for consultation. For these reasons it is believed that a volume containing the rudiments of the subject will be of use to the student and an aid to the teacher. It is also believed that such a work will be of assistance to practitioners.

In preparing this volume the aim has been to bring together in a concise form the fundamental facts in the pathology of the more common infectious diseases of animals, especially those existing in the United States, with which sanitarians and the practitioners of comparative medicine must contend. To this end, the current literature, the reports of the investigations made at various Institutions and Experiment Stations, as well as the standard works on comparative pathology have been freely drawn upon and to all of which full acknowledgment is hereby made.

In order to bring into consideration the clinical value of a knowledge of morbid anatomy, a few of the symptoms or antemortem manifestations have been included. It is hoped that this correlation of symptoms and lesions will stimulate a deeper 
interest in the study of comparative pathology and thus render it of more permanent and practical value for those entering into the practice of veterinary medicine.

In selecting the subject matter care has been taken to avoid, as far as possible, the introduction of results concerning which there are controversies. It has seemed best to deal with those facts about which at the present time there is little or no doubt. After the discussion of each disease a few references to the literature are appended. These are intended simply to bring the attention of the student to a few publications respecting the cause and morbid anatomy, considered in the light of modern etiology, of the disease in question and to a few articles containing the results of original research.

In order not to complicate or unnecessarily expand this text, a knowledge of general pathology and the principles of bacteriology has been taken for granted.

The difficulties involved in the preparation of such a text are both numerous and obvious. The indication of errors or omissions with any other criticisms that will tend to better the volume and increase its efficiency for the student will be thankfully received.

$$
\text { V. A. M. }
$$




\section{TABLE OF CON'TEN'TS.}

PAGE

LIST OF ILLUSTRATIONS. _._.

LIST OF REFERENCE BOOKS ...

INTRODUCTION _...

\section{CHAP'TER I.}

GENERAL CONSIDERATION CONCERNING ETIOLOGY, INFECTION AND SPECIFIC INFECTIOUS DISEASES. (Etiology, Infection, Channels of infection, Wound infection, Bacteria causing wonnd infection, Lesions presumably of wound infection origin that are recognized as distinct maladies, Botryomycosis, Onphalophlebitis, White scours, Infectious suppurative cellulitis, Fistulous withers and poll-evil, Infectious mastitis, A specific infectious disease, The differential characters of a specific infectious disease, Cause for the variation in the course of an infectious disease, Grouping of specific infectious diseases)

CHAP'TER II.

DISEASES CAUSED BY STREPTOCOCCI

General discussion of streptococci _...

Strangles _._ _._. 33

Equine contagious pleuro-pnetumonia _._. 37

Apoplectiform septicaemia in chickens _............... 44

CHAP'TER III.

DISEASES CAUSED BY BACTERIA-GENUS BACTERIUM _... . . . 47

Swine plague.

Hemorrhagica septicaemia in cattle

Fowl cholera............... 77

Anthrax _._.

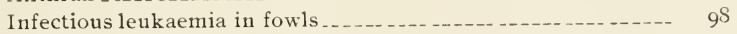

Swine erysipelas _......... IO7

Glanders .................. II

Tuberculosis _.......... I 27

Ovine caseous lymph-adenitis

Asthenia .................... I 67 
CHAPTER IV.

DISEASES CAUSED BY BACTERIA-GENUS BACILLUS _... ... ... 169

Hog cholera

Tetanus _._.

Symptomatic anthrax

CHAPTER V.

DISEASES CAUSED BY HIGHER FUNGI _...

Actinomy cosis _. . . .

Leeches _.

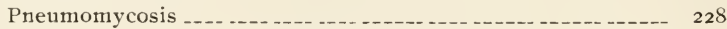

CHAPTER VI.

DISFASES CAUSED BY PROTOZOA _...

Texas fever

Infectious entero-hepatitis in turkeys _._. . 252

Surra - _. - .

Dourine _... . .

CHAPTER VII.

INFECTIOUS DISEASES FOR WHICH THE SPECIFIC CAUSE IS NOT YET DETER IINED ....

Rinderpest ...... - .

Contagious pleuro-pneumonia in cattle .

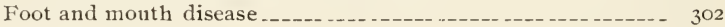

Rabies _...

Diphtheria in fowls _... . . 325

Influenza ...

Dog distemper

Infectious cerebro-spinal meningitis _._. _._. _... 347

Cornstalk disease in cattle

CHAPTER VIII.

A FEIV DISEASES CAUSED BY ANIMAL, PARASITES ...... ... . . 355

The nodular disease of the intestines of sheep and cattle_._._- 355

Nodular taeniasis in fowls ...... . 360

Trichinosis _.

APPENDIX.

DISINFECTION _. 


\section{ILLUSTRATIONS.}

\section{PLATES.}

Bacteria, fungi and protozoa _...................... FRONTISPIECE

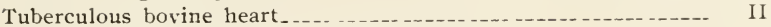

Tuberculous and healthy spleens, pig ........................... III

Tuberculosis of lung, pig ........... IV

Fungus associated with leeches _........ V

Aspergillar pneumonia

Map showing Texas fever line _......... VII

Diphtheria in pigeons ....... VIII

FIGURES IN TEXT.

PAGE

I. Streptococcus of mastitis _.... I6

2. Section of cow's udder......... 17

3. Abscess in partially immunized rabbit _......... 23

4. Morphology of streptococcus _... 28

5. Bacterium of swine plague ................................. 48

6. Lung with interlobular lymph infiltration .................. 53

7. Lung showing emphysema in interlobular spaces__._._._. 54

8. Right lung of pig showing areas affected with swine plague _-_ $\quad 55$

9. Portion of lung showing hemorrhagic interlobular infiltration $-\quad 5^{8}$

10. Anthrax bacteria from an impression preparation........... $8_{3}$

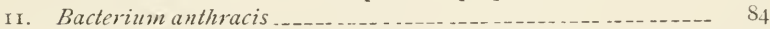

12. Cover-glass preparation from anthrax blood _................ 85

13. Bacterium sanguinarium ................................ 99

14. Bacterium sanguinarium in the liver.................... 99

15. Temperature chart of fowl affected with leukaemia .......... 100

16. Diseased blood in infectious leukaemia

17. Congested liver in infectious leukaemia _._._........... 103

18. Phagocytic action of leucocytes on the red blood corpuscles_- IO4

19. Bacterium of swine erysipelas ....................... 108

20. Bacterium mallei.................................... 115

21. Nasal septum, glanders .......................... I18

22. Farcy, cutaneous glanders

23. Bacterium tuberculosis................................ 131

24. Lymph glands on side of cow's head _.................... I37

25 Dorsal aspect of bovine lungs ........................... 138

26. Bovine tracheal and bronchial lymph glands _............. 139

27. Posterior mediastinal glands _......................... 140

28. Suppurating tubercular focus, cow's lung _.............. I4I

29. Tuberculous omentum, cow ............................. 142 
30. Small tubercular nodules, parietal pleura, cow ............. I43

31. Beginning tubercle in spleen ....................... 145

32. Section of tuberculous lung, cow . _ _..... 146

33. Tuberculous spleen, pig _._._._._._._.

34. Chart showing the effect of cold water on temperature ..._.. I57

35. Non-tubercular temperature reaction _.................. 158

36. Chart showing tuberculin reaction ..._...

37. Bacterium of Preisz.............................. 162

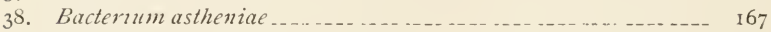

39. Bacillus of hog cholera $\ldots \ldots$

40. Ulcerated intestine in hog cholera

41. Spleen of pig, normal and with hog cholera ........... 177

42. Bacillus tetani _................ I86

43. Bacillus of symptomatic anthrax _.__ _._. 197

44. Ray fungus ...................... 206

45. Actinomycosis, head of steer

46. Section of actinomycotic jaw _....................... 211

47. Actinomycosis of upper jaw .............. 212

48. Actinomycosis in tongue

49. Leeches, lesions in lip of horse ._. . . . . . . . 222

50. Leeches, lesions in lip of horse

51. Isolated nodule from lesion _..._. . _ _ _ 223

52. Club-like ends of hyphae fungus, "leeches" .............. 224

53. Section through nodule, "leeclies"' ............ 225

54. Piroplasma bigeminum _........................... 238

55. Coccus form of Piroplasma bigeminum _._._._._. 239

56. Texas fever parasite in blood of kidney _._. 239

57. Coccus form of Texas fever parasite in kidney ........... 240

$5^{\mathrm{S}}$. Capillary of heart showing invasion of corpuscles with Piro-

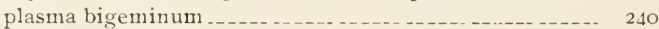

59. Adult male tick, Boöphilus bovis _...

6o. Bull suffering from Texas fever ..................... 242

6r. Adult female tick, Boöphilus bovis _... . . .

62. Eggs and young tick, Boöphilus bovis _...

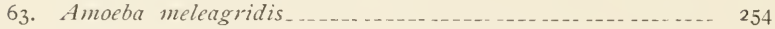

64. Caeca of turkey with lesions of entero-hepatitis _.......... $25 \mathrm{~S}$

65. Caecum showing ulcers in entero-hepatitis ............. 259

66. Liver of turkey affected with entero-hepatitis _............ 260

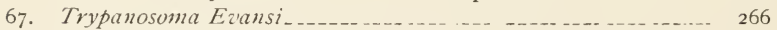

68. Plotograph of Trypanosoma in blood .................. 267

69. Nodular disease in sheep, cross section of intestine _....... 355

70. Cross section of worm nodule ......................... 358

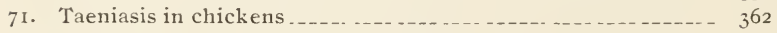

72. Sections showing nodule and worm .................... 363

73. Encapsuled Triclinella spiralis .......................... 365 


\section{A LIST OF REFERENCE BOOKS.}

BotLEy ET REYNAL. - Nouveau Dictionnaire pratique de Médecine de Chirurgie et d'Hygiène Vétérinaires.

CADÉAC.-Encyclopédie Vétérinaire.

DiEckerhofF, - Lehrbuch der Speciellen Pathologie und Therapie für Thierärzte.

ElLENBERGER, SchüTZ UND BAUM.-Jahresbericht über die Leistungen auf dem Gebiete der Veterinär-Medicin.

FI.EMING. - A manual of veterinary sanitary science and police.

Friedberger UND Fröhner.-Lehrbuch der Speciellen Pathologie u. Therapie der Hausthiere.

Galtiter. - Traité des Maladies Contagieuses et de la Police Sanitaire des Animaux domestiques.

KiTT.-Lehrbuch der Pathologischen Anatomie der Hausthiere.

LAw.-Veterinary Medicine.-(Especially Vol. IV.)

LUbarsch Und OSTERTAG. - Ergebnisse der Allgemeinen Pathologie und Pathologischen Anatomie des Menschen und der Tiere.

OSTERTAG.-Handbuclı der Fleischbeschau für Tierärzte, Ärzte und Richter.

REYNAL. - Traité de la Police Sanitaire des Aninıaux Domestiques.

SCHNEIDEMÜH L. - Lehrbuch der vergleichenden Pathologie und Therapie des Menschen und der Hausthiere.

WALLEY.-Tlie four bovine scourges.

For bibliography on all medical subjects, see Index-catalogue of the library of the Surgeon-General's office.

Annual Reports, Special Reports and Bulletins on Animal Diseases issued by the Bureau of Animal Industry, U. S. Department of Agriculture, Washington, D. C.

Proceedings of the American Veterinary Medical Asscciation.

The Bulletins on Animal Diseases issued by the various State Agricultural Experiment Stations. 


\section{INTRODUCTION.}

An elementary treatise on the pathology of the infectious diseases of animals - a treatise that states briefly, clearly and comprehensively all that is known, and excludes all that is not known-has long been needed not only by the students who are beginning this interesting subject, but by members of the veterinary profession who, as practitioners, investigators or teachers, wish to learn in the shortest time the present condition of our knowledge. A work which supplies this need will be welcomed and appreciated.

There are few subjects more important to Americans than a thorough comprehension of the infectious diseases of animals. An enormous amount of money is invested in the domesticated animals in the Inited States, and the security of this investment depends very largely upon our ability to protect these animals from infectious diseases. There are many diseases of this class which spread among animals as smallpox, bubonic plague or cholera spread among mankind; and it requires a thorough knowledge of all the characteristics of such diseases to guard against them, to recognize them when they appear or to control them.

For a period already too long, exact knowledge of these diseases has been confined to a comparatively small number of men ; but with the great property interests at stake it is extremely desirable that this information should be distributed, that not only responsible officials but every practitioner should share it. With not far from three thousand million dollars worth of farm animals in this country, and with a single disease 
that sometimes destroys a hundred million dollars worth of property in a year, it is not difficult to see the value of that precise knowledge which is required to deal promptly and efficiently with these plagues.

It is a narrow and incorrect view to hold that the farmer who owns these animals is the only one who suffers from the ravages of the diseases which destroy them. Animal products constitute a large part of the national food supply. If this food supply is diminished, made dearer and more difficult to obtain, want, misery, disease and death among mankind increase. At first the effects of a scarcity of the food supply may be almost inappreciable and felt only by the very poor; but as the conditions of famine are approached, suffering is multiplied and intensified until whole communities are prostrated or destroyed. An abundant supply of wholesome and nutritious food is, therefore, an essential condition of the welfare and prosperity of a people.

The great commercial operations of nations also depend to a great extent upon the good condition of animals. When all of the horses are disabled by an epizoötic, as they have been on rare occasions by influenza, the delivery of purchased goods has nearly ceased, the shipments of flour, iron, machinery and other products have been temporarily arrested and business has been almost at a standstill. Again, it should be remembered that we export annually from the United States forty million dollars worth of live animals, one hundred million dollars worth of meats, fifty-five million dollars worth of lard, tallow and other animal fats, and nine million dollars worth of dairy products. Let this traffic be stopped by a shortage of supplies or by prohibitive orders of other nations on account of the unrestrained prevalence of infectious diseases, and the earnings of steamships, and rail-roads, and banks, and commission houses, are at once diminished ; men employed in these 
enterprises are discharged, and in innumerable ways the welfare of people who have no part in the ownership of any of these animals or their products is affected.

Finally the most serious of all, is the case in which the animal plague, in addition to being destructive to animal life, is communicable to and fatal to man, as is true of anthrax, rabies and tuberculosis. Such diseases destroy property, disturb business, lessen the food supply, and directly threaten human life. What more is needed to impress upon the reader the supreme importance of studying and understanding the infectious diseases of animals?

The pathology of these diseases is in itself a large subject. Investigations concerning it have extended over more than a century of time and the literature of the subject is enormous. To concentrate this knowledge, to select the truth and discard the errors, and to develop a concise and systematic treatise is a task of great magnitude and one which has required much labor and thought. The writer has not seen the manuscript, but, from his long and intimate acquaintance with the author, he feels sure that the work has been patiently, carefully, intelligently and thoroughly done, and that it will be favorably received.

D. E. SALMON. 


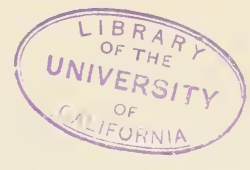

CHAP'TER I.

\section{GENERAL CONSIDERATION OF ETIOLOGY, INFECTION AND SPECIFIC INFECTIOUS DISEASES.}

s. Etiology. The development of the science of bacteriology, together witl a knowledge of the parasitic protozoa, has denonstrated that a large number of the infectious diseases are the direct result of the invasion of the animal body by certain species of microorganisws. A specific etiology which teaches that for each of the various epizoötics we have a single, definite cause has become recognized and accepted by all pathologists. Although there are a number of distinct diseases for which such a specific agent has not been found, the evidence in the very nature of the maladies, is conclusive that for each of these such an etiological factor exists.

In studying the pathology of infectious diseases the idea of a definite and adequate cause should be kept in mind. It is no longer justifiable to attribute them to an unfavorable environment, poor hygiene, or improper sanitation, conditions which may aid the specific cause but which cannot supplant it. It is often a troublesome task to differentiate between the morbid affections, often fatal in their results, brought about by improper care and food, and the maladies dependent upon a specific cause. The reason why many of the former theories accepted unsanitary conditions, certain kinds of food or other similar agencies as the etiology of distinct, infections diseases, is found in the fact that the infectious microorganisms were and still are often transmitted to the individual through such channels. It is important, therefore, that the limitations of both the exciting cause and the enviroument should be fully taken into account. Although for certain diseases such as rinderpest and rabies we do not know just what the specific 
cause is, the fact that its location in the body of the diseased animal is known, that with these morbid tissues the disease can be produced in healthy animals and that without these definite infections, no matter what the surroundings are, they cannot be made to develop, argues against extraneous conditions as exciting causes.

The mysteries which formerly surrounded the origin, the course and the disappearance of animal plagues have in a large degree been cleared away and in their place we are confronted with the problems involved in the life history and the possibilities of invading microorganisms. In fact during the last few years the biological sciences have been brought into immediate use by the pathologist. Etiology has become permanently linked to microbiology so that in seeking for the specific cause of an infectious disease we look for some species of organic life which may belong either to the animal or to the vegetable kingdom. The fact that certain animals and plants have become, if they were not in the beginning, parasitic on other larger and higher forms of life has long been recognized, but the idea came later, that the various infections giving rise to a wide series of phenomena, known as symptoms and morbid anatomy, were actually and simply the results of the invasion of the individual with living microscopic plants (bacteria) or animals (protozoa). It is likewise true that for many of the disorders consisting of changes recognized in the terms of general pathology, the cause may be found in the conditions of life under which the individual has been forced to exist. Etiology, therefore, in a broad sense, includes both the infecting or parasitic microorganisms which cause infections and produce the specific infectious diseases and poor hygienic, unsanitary conditions and physical forces which may produce non-specific morbid changes often sufficient to cause death.

2. Infection. The term infection has come to be generally understood to mean the entrance into the animal body, from without, of living microorganisms capable of multiplying within the living tissues and of producing in consequence thereof a local or a general diseased condition or the death of the individual. The invading microorganisms may belong to any 
one of the three great groups of microscopic life, namely, bacteria, higher fungi, or protozoa. It is customary and convenient, if not altogether logical, to limit the term microorganism to these forms excluding altogether the entozoa and other animal parasites most of which are not microscopic in size.

A diseased condition produced by substances not capable of reproducing themselves as, for example, organic or inorganic chemical compounds, is an intoxicative process. In an infection, the immediate cause of the symptoms and morbid changes in the tissues is an intoxication due to the action of the metabolic products (toxins) of the invading microorganisms. The theories of their mechanical interference with the normal functions of the body or that they absorb the nutriment thus depriving the tissues of necessary food wait for demonstration. The results of infection vary in their manifestations. If the invading organisms remain at the point of entrance and produce local tissue changes, the condition is spoken of as a wound infection ; if the invading bacteria become widely distributed in the circulation and tissues, the condition is known as septicaemia; if the infecting bacteria remain at the point of entrance, multiply there elaborating a toxin which is absorbed and which causes symptoms and possibly death, the condition is a toxaemia; if the invading organism happens to be that of a specific disease, such as the bacterium of anthrax, giving rise to a definite series of symptoms and lesions, the affection is designated a specific, infectious disease. Through the agency of metastasis, invading microorganisms may be carried from the point of introduction to other parts of the body where they may become localized, multiply and give rise to any one of many forms of lesions. It may happen that the point of entrance is so obscure that the resulting morbid changes are not easily traced to an external infection. There are many illustrations of this in comparative pathology such, for example, as suppurative cellulitis. For convenience in discussion, infections may be divided into the two clinical groups, namely, wound infections and specific infectious diseases although in certain instances they cannot be separated. 
In arriving at a clear understanding of the nature of infections, it is well not to be too closely circumscribed by classifications. It is better to look upon them as a series of processes going on in the animal world due to the activities of infecting or parasitic microorganisms. In other words, the lesions following an infection or a specific infectious disease are simply the results of parasitism.

In the study of the various forms of infection in the lower animals, lesions have been found to contain, apparently as their causative factors, bacteria which suggest at least that certain of the supposed saphroplyytic organisms may, under certain conditions, become parasitic and canse infections resulting in more or less local or generalized disturbance. Many lesions seem to be produced by bacteria which are harbored normally upon the skin. When these organisms are introduced by accident into the living tissues they multiply and acquire, if they did not already possess it, the power to produce tissue changes. We cannot, therefore, dismiss the subject of infection without a passing consideration of the possible etiological significance, under certain conditions, of many ordinarily considered harmless species of bacteria with which the animal body is constantly surrounded. In the search for the cause of many lesions supposed from their nature to be infectious, or in applying methods for their prevention, it is well to take into consideration all microorganisms which might possibly be the causative factors and not limit the search to the detection of the already recognized pathogenic species. Recent investigations point to the conclusion that frequently domesticated animals suffer as the result of the invasion of bacteria at present not listed among the pathogenic microorganisms, and what is true in this regard for bacteria, may be hypothetically applied to the higher fungi and to the protozoa.

$\$ 3$. Channels of Infection. There are a number of ways by which microorganisms may be introduced into the living tissues of the animal body. The more common of these are as follows, namely :

(i) Through the digestiz' tract. Bacteria gain entrance into the tissues from the digestive tract where they have been 
brought with the food or water. Hog cholera infection is a good illustration of this mode of entrance.

(2) Through the respiratory tract. Bacteria are taken into the lungs where they are brought with the inhaled atmosphere. Pulnonary tubercular affection is often brought about in this way.

(3) Through abrasions of the skin. The wide distribution of bacteria in nature renders it highly probable that in all wounds of the integument microorganisms will reach the fresh tissues. They may come from the cutting or tearing implement, the particles of dirt which may fall into or upon the cut surface, or from the ducts of the glands of the skin itself. It may happen that the fresh tissues thus exposed are infected with one, or, with several species of bacteria. It nuay be that one or 1110re of these species 111ay be destroyed by the living juices of the body or by the leucocytes, or, again it is possible that, from their saprophytic nature, they may not be able to nultiply in this new environment; in either case the infection is of no significance and clinically would not be recognized. It may happen that only one species of the infecting bacteria multiplies and produces the nuorbid changes. This would be a single infection. If, however, two or more species co-operate in the production of the lesions, it is called a mixed infection. This term is often used to designate the condition where one species may be responsible for the tissue changes although other bacteria are present but in an accidental or passive way $(\$+)$.

(4) Through the generatize organs. Infection of the reproductive organs takes place in certain instances where they are the seat of the disease. This is especially true in case of maladie du coit.

(5) Through the agency of insects. Some insects carry the virus of certain diseases from the infected and introduce it into the susceptible individuals. Thus the mosquito carries the plasmodium of human malaria, the cattle tick the piroplasma of Texas cattle fever and flies are often the introducers of pathogenic bacteria, such as those of anthrax $(\$ 76)$.

(6) Transmission of the vinus from the parent to the foetus. Occasionally the young of diseased parents are born infected 
with the disease with which one or both of its parents were suffering. In these cases the specific bacteria were transmitted either from the sire at the time of coition, or later to the foetus in the uterus from the dam. It is important not to confuse these rare cases with those in which the offspring are born uninfected but subsequently contract the disease. Many of the so-called hereditary diseases are the result of post natal infection.

$\$ 4$. Wound infection. Wound infections are the direct results of the entrance of certain microorganisms into traumatisms and operative incisions. They fall very naturally into two classes namely, (I) those producing acute or more chronic inflammatory processes usually leading to suppuration and finally healing by granulation, and (2) infections which may in the beginning appear like the first or, cause so little disturbance as to be unnoticed at the time, but sooner or later result in a local or remotely situated lesion or lesions. Frequently these are recognized as distinct diseases although in some cases, such as scirrhous cord, the origin is easily traced to an operation where infection was possible. In this second group of wound infections there are in addition to the scirrhous cord or botryomycosis, infectious cellulitis of cattle and sheep and still other disorders which may not seem to be dependent upon wound infection but which the results of recent investigations suggest as their primary cause. These affections will be treated separately in subsequent paragraphs but their relation to wound infection renders them worthy of note in this connection.

A third class should be mentioned, namely, those specific diseases such as tetanus and symptomatic anthrax where the virus is supposed always to be introduced through a wound either in the skin or mucous membranes.

$\$ 5$. Bacteria causing wound infection. A large uumber of species of bacteria and a few fungi are included among the organisms which are known to produce wound infections in animals. Usually, however, the forms encountered are micrococci, especially those belonging to the staphylococcus group, streptococci, a few bacilli, especially those belonging to 
the colon group, and a few species of the genus pseudomonas Fungi and protozoa are rarely found in wound infection lesions. Many of the specific pathogenic bacteria may be introduced through wounds ( $\leqslant 3$ ). As a rule, it seems to be true that in the domesticated animals as in man the pyogenic bacteria are the most common and important wound infecting microorganisms. In open wounds these are, of course, associated with a very large number of ordinary saprophytic bacteria. Frequently in closed lesions nonpathogenic organisms are present in addition to the seemingly causative factors. It is worthy of note, that it appears to be impossible to predict from the general character of the lesions, the kind of bacteria which are producing them except it be in case of certain specific infections like tuberculosis or actinomycosis.

\$6. Lesions presumably of wound infection origin that are recognized as distinct maladies. It has already been stated that the lesions following wound infections may be brought about by a variety of bacteria and also that certain of these diseased processes are sometimes recognized as distinct maladies. There are a number of affections which belong to this class. From some of these, such as botryomycosis, a supposedly specific organism has been isolated and described. A number of workers, however, have found that other bacteria may prodnce apparently the same morbid conditions. The nore important of the affections which seem to be directly traceable to wound infection will be briefly described in the light of recent investigations. It should be stated, however, that the amount of work that has been done on these subjects is not sufficient to render the results in any. way conclusive, but rather to suggest the lines along which valuable and conclusive findings may be expected in the future.

\$ 7. Botryomycosis. This name has been given to a variety of lesions found more commonly in the horse but occurring also in cattle, swine and other animals. The thickened spermatic cord (scirrhous cord) which sometimes follows cas tration is the most common form of this disease. Practitioners often designate as botryomycosis certain closed abscesses occurring in the subcutaneous or intermuscular tissue. Abscesses 
and nodules found in the internal organs have been included under this caption. Several investigators have isolated from these lesions a species of microorganism which appeared to stand in a causative relation to them. It was first described as Zoogloea pulmonis equi, in 1870 , by Bollinger, who found it in the nodules in the lungs of a horse. More recently he has renamed it Botryococus ascoformans. Rivolta designated it Discomyces equi. Rabe proposed the name Hicrococcus botryogenes and Johne has called it $\Pi$. ascoformans. The results of other investigators throw some doubt upon the specific nature of these lesions. Kitt, Hell, deJong, Gay and others have found in them micrococci which do not differ from $M$. pyogenes aureus. The writer has failed to find $/$. ascoformans but has isolated in its stead pyogenic micrococci and streptococci. In one very interesting case of thickened cord, the writer found masses of a fungus resembling that of actinomycosis within the pockets of spongy tissue sprinkled throughout the thickened fibrous cord. Bacteria were not found in this case. In the closed abscesses in the connective tissue pyogenic bacteria have been found, excepting in certain cases of long standing where the cultures gave negative results. Investigations which have been made into the bacterial flora of the skin of the horse show that pyogenic bacteria are frequently present in the deeper layers of the epidernis, in ducts of glands and about the hair shafts. With the possibility of infection from the integument plus all the other chances of having nembers of this and other groups of bacteria introduced into the body there seems to be abundant opportunity for infection by a variety of species. The evidence at hand points to the conclusion that botryonycosis is the result of wound infection, and that several species of microorganisms are capable of producing it.

8. Omphalophlebitis. This affection which is commonly called navel-ill, consists of suppurative lesions in young animals caused by pyogenic bacteria. In the horse they are most often localized in the joints of the limbs. In certain other species the lesions are quite as likely to be situated elsewhere in the body. In some cases the morbid changes are restricted to subcutaneous and interınuscular suppurative cellulitis. 
The infection takes place in the umbilicus. As the cord is serered in the field or stable many species of bacteria may gain access to the end of the exposed, and freshly severed cord. In the colt, a streptococcus seems to be the nost common species of bacteria capable of producing the joint abscesses. In the lanbb, a variety of the colon bacillus has been associated apparently as the etiological factor with the subcutaneons cellulitis. The lesions resulting from naval infection illustrates in a most excellent manner the extent to which certain pyogenic bacteria gaining access to the body may extend by metastasis to places remote from their entrance and produce diseased foci.

In cases of navel-ill, the umbilical vein contains a large number of bacteria. The writer has found that in colts very few if any of the bacteria (streptococci) producing the joint lesions could be found in the parenchymatous organs. Occasionally one or two of nnany tubes of media inoculated from the liver would develop into cultures of the infecting organism. In this disease, where many species of bacteria cone into competition, one is impressed with the fact that seemingly very few of the ordinary bacteria are capable of gaining an entrance into the circulation or, at least, are possessed of vital powers sufficient to resist the destructive forces of the living animal body. A brief description of a case with the bacterial findings will illustrate this point. The case referred to was from Dr. Willians' clinic.

A colt, about three weeks old. It was in good condition and seemed to be perfectly well excepting for the diseased joints. It was killed for examination. The umbilical vein, from the umbilicus to the liver, was distended with blood, pus cells and bacteria. All of the internal organs appeared to be normal. In both knee joints and in one liock joint, there was extensive suppuration. A bacteriological examination showed the umbilical vein to contain many species of bacteria, anong which nua be mentioned B. coli communis, Micrococcus pyogenes aureus and a streptococcus. One of several tubes of media inoculated from the liver developed the streptococcus, the others remained clear. All media inoculated from the heart blood, spleen, kidney and glands remained sterile. All of the media inoculated with the pus from the diseased joints gave pure cultures of the streptococcus. 
\$. White scours or diarrhoea in calves. This is a disease affecting calves from a few hours to as many days old, with a mortality ranging from 50 to 90 per cent. The investigations which have been made in this country, especially those at the New York State Veterinary College, have suggested that it is due to certain forms of the colon bacillus. In these investigations characteristic lesions were not found, the calves presenting simply a general anaemic condition.

Recently, Nocard has reported the results of his investigation of apparently a similar disease of calves in Ireland. He found that they usually die during the first week. In the more chronic cases, lung lesions were found. His inquiries tend to show that this is primarily due to a wound infection. He states in his report concerning the nature of this disease that it usually lasts from 3 to 6 days and is characterized by an intense intestinal discharge. The discharges are always of the nature of a diarrhoea, white, and frothy. The calves lose flesh rapidly, their flanks are hollow, abdomen retracted, back arched, eyes sunken, hair dull and staring; they make violent expulsive efforts, the nose is hot and dry with slight discharge of mucus, and the temperature is elevated.

In other cases, but not so commonly, the symptoms are less severe and recovery seems to take place but most of the calves die several weeks later with pulmonary lesions.

Nocard also states that it is not rare to see, in the cases, the discharges mixed with blood in various quantities. In more chronic forms it is not rare to observe acute, multiple and very painful arthritis.

The lesions found at the autopsy vary according to the conrse of the disease. Usually the umbilicus is large and the umbilical blood vessels have indurated walls, and contain blood clots which may be soft and purulent. Bloody extravasations are observed, sometimes very extensive, along the umbilical vessels and the urachus, extending sometimes to the posterior third of the bladder.

In rapidly progressing cases, lesions of true hemorrhagic septicaenia are found. All the organs are congested; their surface is covered with petechiae, ecchymoses or sub-serous 
blood infiltrations; the capillary network of the peritoneum, the omentum, the pleura and the pericardium is very much injected.

Nocard describes the lungs and articular lesions as follows:

"The lungs are rarely entirely healthy ; most often they present here and there little diffuse centers of catarrhal pneumonia, nodular broncho-pueumonia or only of atelectasis. The lesions are nuch more constant, extensive and dense when the animals have resisted longer; they represent then the transition between the simple atelectasis of the beginning and the suppurative lesion of lung disease."

"Articular lesions, when they exist, are very interesting. The periarticular tissues are infiltrated with yellowish and somewhat gelatinous liquid; the synovial serous menıbrane is covered with a rich vascular injection, which seems to extend to the borders of the cartilages of the articular surfaces; the culs-de-sac of the synovials are distended by a great quantity of thick and limpid synovia, strongly yellow or reddish in color, and in which more or less dense and abundant clots of fibrinous exudate are floating. When the lesion is older, instead of synovia, there are thick, dense, and firm fibrinous exudates, which fill the culs-de-sac of the serous membrane and are infiltrated between the articular surfaces. In these cases the lesion resembles exactly those of peripneumonic arthritis of suckling calves."

He found a microorganism (pasteurellose) in the organs and blood of the calves, with which he was able to produce the disease. After convincing himself that he had found the cause, he sought for the source of infection which he found in the umbilicus. He advances three suppositions concerning the time and mode of entrance : 1 . intra-uterine, 2. vaginal, 3. after delivery when the calf drops on the ground or floor and when the ruptured cord comes in contact with the fecal matter or dust of the stable. The latter he believes to be the correct method. His conclusion concerning infection after delivery may be summarized in the following observation which he makes:

"We have witnessed in a well kept farm a case of labor in a cow. She was in an ordinary barn. Nothing had been pre- 
pared to receive the new-born. The calf was dropped on a bedding soiled with feces; he only fell back a little, and there during 15 or 20 minutes, we watched him making his efforts to get up, falling back here and there to the right and to the left or on his belly, dragging the stump of his cord on the ground, in the urine, or even in the fecal matter. It was only after the mother had well licked her little one, well covered with salt, that the cord was ligated. I am not sure that the umbilicus was cleaned.

White scours is ordinarily the result of umbilical infection which takes place at the time of delivery, by the way of the wound made by the rupture of the cord."

He states that this trouble can be prevented if the person in charge of the animals at the time of their delivery takes certain precautions to prevent infection. *

This disease described by Nocard does not seem to differ in many respects from the diarrhoea in young calves in this

\#"1. Cows ready to calve should be provided with dry and clean bedding until after the birth of the calf.

2. As soon as labor sets in the vulva, anus and perineum should be cleaned with a tepid solution of lysol in rain water; twenty grammes of lysol for each litre of water. The vagina should also be cleaned by injecting with a large syringe a great quantity of the same tepid solution.

3. The calf should be received on a clean cloth or at least upon a thick fresh bedding not soiled by urine or feces.

4. The cord should be tied immediately after birth with a ligature previously kept in a lysol solution, and the cord amputated below the ligature.

5. The stump of the cord and the umbilicus should be washed with the following solution :

$\begin{array}{ll}\text { Rain water, } & 1 \text { litre. } \\ \text { Crystals of iodine, } & 2 \text { grammes. } \\ \text { Iodide of potassium, } & 4 \text { grammes. }\end{array}$

6. The disinfection of the umbilicus and the cord should be followed by coating the umbilicus with a solution consisting of
Methylic alcohol,
1 litre.
Crystals of iodine,
2 grammes.

7. The operation should be closed, after the alcohol has evaporated, by coating the cord and umbilicus with a thick layer of iodine collodion ( 1 percent) applied with a brush. Once the collodion is dried, the calf may be left to the care of its mother." 
country. Ward and Fisher are making careful tests of his method witl quite satisfactory results. In our cases the lung complications did not occur. The important finding of Nocard should stimulate further investigation into this most important trouble in this conntry. As the remedy which he recommends and which has given good results is simply one to prevent infection of the ruptured cord, the conclusion tends to the wound infection origin of this disease.

$\$$ Io. Infectious suppurative cellulitis. Cattle and sheep suffer from more or less extensive inflammatory conditions of the subcutaneous tissues especially of the lower extremities. Frequently the morbid process extends beneath the hoof causing it to slough or to undergo resulting disintegration changes. When this condition exists, the affection is usually called "foot rot." If the inflanmatory process attacks the skin also, the condition is often designated erysipelas. If it becomes circumscribed, resulting in a local suppuration, an abscess or an ulcer is the result. The investigations which have been made concerning the cause of these lesions point to the conclusion that they result from an infection, probably througl some slight abrasion of the skin. Thus far, the results show streptococci* to be the etiological factors in the majority of these cases. It frequently happens that a number of animals subjected to the same conditions are attacked at the same time, giving rise to a condition resembling an epizoötic. In cattle, cases studied by the writer, - the lesions were, within certain limits, uniform in all of the affected animals. Ustrally but one foot or leg was attacked, although there were numerous exceptions. The first sympton noticed was a swelling, which usually appeared in the lower part of the leg, most often in the pastern. In some animals the swelling was restricted to a small area, but often it extended up the leg to and even above the knee or the hock joint. 'There was evidence of pain. As the inflammatory process continued, the subcutaneous tissue became

* Lucet has reported the results of bacteriologic examinations of fiftytwo abscesses in cattle. From nine of these streptococci were obtained in pure culture, and in ten cases they were associated with other bacteria. - Annales de l'Institut Pasteur. I'ol. VII (IS93) p. 324. 
indurated, the skin thick and dry, and later it would crack, usually but not always, below the dew claws, and a thick creamy pus would be discharged. After discharging, the swelling subsided and the normal condition was rapidly restored. The time necessary for the suppurative process and recovery to take place varied in different animals, but as a rule from ten to fifteen days were required. The exceptions were largely in those cases where the inflammatory process extended down to the coronary cushion. In these there was more or less sloughing of the hoof. It is in these cases that the disease is most serious.

In the so-called foot-rot of sheep, we have, in the cases which have come to our notice, conditions similar to those found in the cattle which were suffering from suppurative cellulitis. It may be of interest to cite a specific case with the result of the bacteriological examinations.

May, I899, two sheep that were suffering from so-called foot-rot were brought to Dr. Law's clinic. They came from a large flock in which forty or more animals were reported to be similarly affected.

No. 1. An adult female in very poor condition. All four feet and legs were affected and the nails on one foot were quite loose. There was a purulent discharge froni openings either between the claws or in the skin just above the hoof. The microscopic examination of the pus from this opening showed a number of bacteria but streptococci were especially numerous. They were not isolated in pure culture. The left $\mathrm{knee}$ was badly swollen and from the lower side of the swelling there was a discharge of thin purulent substance which contained streptococci in large numbers. A few other bacteria, mostly micrococci, were associated with them.

No. 2. An adult female, black, emaciated, but in much better flesh than No. 1. The two fore feet and the left hind one were affected. The hind foot and the right fore one were discharging. The left fore foot was badly swollen above the hoof but the swelling did not extend high up the leg. Fluctuation was marked. The skin was shaved, washed, disinfected and the abscess opened. A thick creamy pus was expressed. From this a number of tubes of media were inoculated and in each a streptococcus developed in pure culture. The pus from the discharging feet contained a streptococcus with other bacteria. The feet were treated locally with disinfectants by Dr. Law. In recovering there was consid- 
erable thickening of the interdigital tissue. In this case the suppuration had not extended under the nails.

$\$$ II. Fistulous withers and poll-evil. Recent investigations indicate that these very common and troublesome local diseased conditions are either directly or indirectly the result of bacterial invasion. This conclusion is tentatively drawn from the fact that the bacteriological examinations made from the pus and from recent lesions in these affections invariably reveal the presence of streptococci or micrococci, or both. Gay found a streptococcus in each of seven cases of common fistulous withers and in two cases of poll-evil. It was invariably associated with a micrococcus. He found in five cases of deep seated shoulder abscesses $M$. pyogenes aureus only. It is instructive to note, that bacteria closely resembling this organism has frequently been found in the deeper layers of the skin. The mechanical injuries commonly attributed as the primary cause consist usually of little more than skin irritation from ill fitting harnesses, saddles or from blows. While these are mechanically not extensive, they are sufficient to liberate into the juices of the subjacent tissues the bacteria deeply seated in the integument. The inflammatory process leading to suppuration, the formation of fistulæ, the new formation of fibrous tissue in the affected parts, and even the bone necrosis occasionally seen are all possible and rational results of the activities of the pyogenic bacteria found in the lesions. There is nothing in their character to suggest causative agencies other than microorganisms. The changes involved in the inflammatory process, the deposition of fibrous tissue, and the abscess formation all belong more properly to general pathology and need not be discussed here. These affections are mentioned in this connection simply because the accumulating eridence tends to strengthen the working hypothesis that they are the result of bacterial invasion.

I2. Infectious Mastitis. Cattle suffer frequently from an acute inflammation of the udder as the result apparently of an invasion by a number of bacteria. The results of the investigation of this affection thus far reported suggest that the form which is transmitted from animal to animal is caused by 


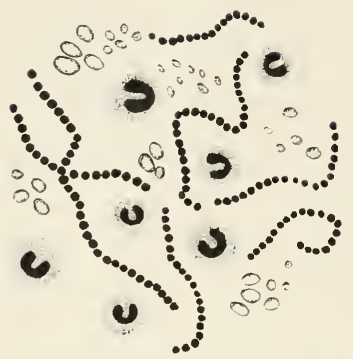

Fig. I. Streptococcus from a case of infectious mastitis. a streptococcus (Fig. I). It is, however, difficult to distinguish between this affection and those caused by certain other bacteria. It seems likely that many cases are primarily brought about by mechanical injuries which render possible the entrance into the fresh tissues of the bacteria of the skin or of the milk ducts. Other cases may be due to infection through the teat of bacteria capable of producing, by means of their metabolic products, the inflammatory condition without a distinct injury to the nucous membrane. The forner view that there was a sphincter muscle near the base of the teat which closed the duct sufficiently to prevent the entrance of bacteria to the secreting portions of the gland was not well founded upon anatomical facts (Fig. 2).

The acute and more chronic inflammatory affections of the udder fall very naturally into two groups, namely, (I) those in which the parenchyma is most affected and (2) those in which the stroma or fibrous tissue is inrolved. The form of mastitis more frequently encountered as an infectious (transmissible) disease is characterized by very marked changes in the milk accompanied by the usual symptoms of parenchymatous inflammation of the gland itself. The discharge from the udder usually contains flaky masses held in suspension in the clear or perhaps cloudy serum. The color varies, and occasionally the mass is blood-stained. The nicroscopic examination shows the presence of agglutinated fat globules, pus cells, and often red blood corpuscles.

A number of bacteria* considered of more or less etiological

*Among the bacteria which have been found in udder trouble and described as the possible or perhaps the more probable cause the following species may be mentioned:

Bacterium phlegmasice uberis, Streptococcus agalactice contagiosa, Staphylococcus mastitidis, Galactococcus versicolor, G. fulvus, G. albus. 


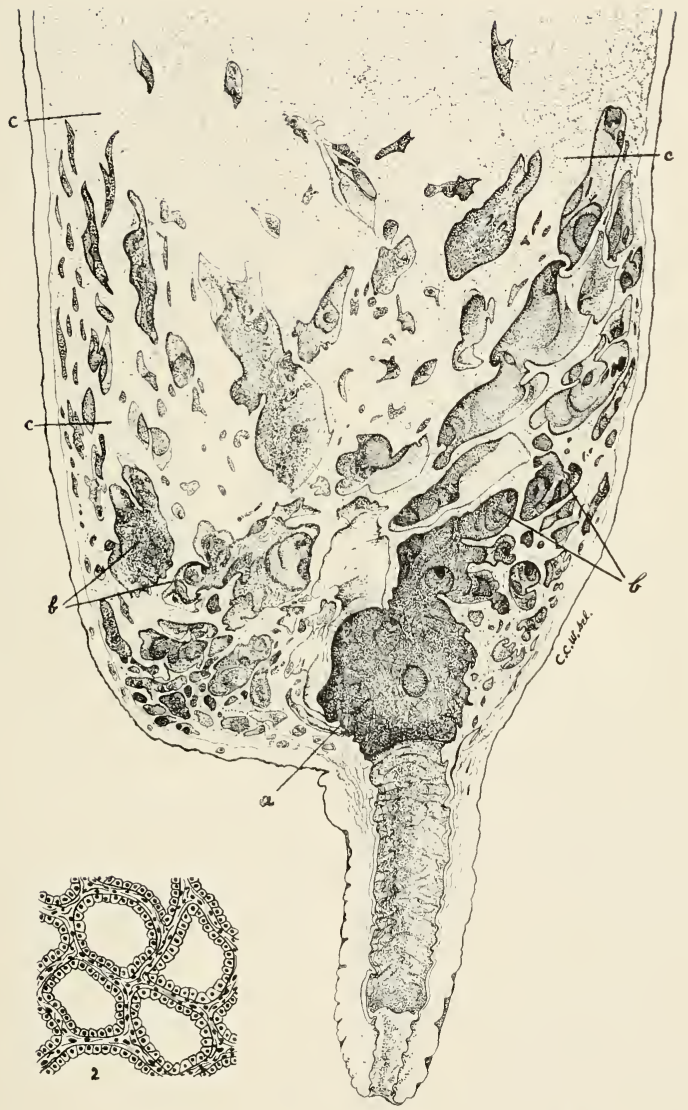

FIG. 2. Section of cow's udder through one teat $(a)^{\circ}$ cistern, $(b)$ larger milk ducts, (c) secreting portion of mammary gland. 2, drazving of secreting portion of gland entarged. 
value have been found associated with lesions of doubtful specific origin. The results of Kitt, Nocard, Mollereau, Guillebeau, Zschokke, Bang and still others in which a Bacterium, a Micrococcus, a Staphylococcus, and a Streptococcus have been found and reported as standing in a causal relation to the trouble, indicate that a variety of microorganisms are active in producing those affections which are frequently grouped without distinction as infectious mastitis. The review of much of the literature on this subject, shows that a number of cases reported as infectious were isolated or sporadic ones, $i$. e., they were in dairies where the disease did not spread to other animals. While these may be truly infectious in their nature they should be differentiated from the rapidly spreading phlegmons which are easily recognized as infectious (contagious).

If we take into account the variety of anatomical changes which have been described in the various udder affections, we can reasonably admit that different agencies may have been instrumental in their production. The various species of bacteria which have been isolated from the udder lesions may very likely have been of etiological importance in their respective cases.

Already the facts have been pointed ont, that the udder is normally more or less extensively invaded with bacteria and that certain species of bacteria seem to persist in the milk ducts of the glands when once they become lodged there. If these results apply to cows generally as rigidly as they did to those examined, an explanation for the presence of a variety of bacteria in the affected udders is not difficult to find. Whether these particular organisms, under certain conditions, would become primarily responsible for udder disease is not known. The evidence suggests that a number of the bacteria, heretofore described as the cause of mammitis, were in the affected glands by virtue of their presence in the normal udder. Concerning these points additional investigations are much needed.

The writer has examined the secretions from two quite serious outbreaks of mastitis. In the first, the milk was drawn in sterile bottles after the udders and the hands of the milker 
had been thoroughly washed in a I to Iooo solution of corrosive sublimate. In all, there were eight samples of milk taken from as many different cows. In six of the eight specimens streptococci appeared in pure culture. In the other two cases micrococci were associated with the streptococcus. In the second outbreak, the milk from four diseased udders was drawn with aseptic precautions directly into tubes containing slant agar and promptly sent to the laboratory where it was carefully examined. From two cases pure cultures of streptococci were obtained while those from the others were impure. The streptococci obtained from the twelve cases appeared to be identical and the clinical aspect of the disease in the different animals was the same.

In a dairy that was under close observation by Ward, one cow was found to be troubled in one quarter of the udder with an inflammatory process which produced thickened masses in the blood-stained milk. From this milk a streptococcus was isolated in pure culture. It could not be differentiated from the one isolated from the cows in the outbreaks mentioned. Another cow in this herd was found to have her udder permanently infected with a streptococcus. Another animal in the same dairy suffers repeatedly from actite streptococcus mastitis.

There are a large number of morbid conditions more or less frequently encountered in domesticated animals which seem to be due to infection of some kind but which are not demonstrated to be of such an origin. These will continue to be attributed by some to infection and by others to various general causes until the truth concerning their etiology is revealed.

13. A specific infectious disease. A specific infectious disease is the result of the multiplication within the animal body of a single species of microorganisms. The lesions may be localized or general but the cause producing them is always the same. Thus Bacterium anthracis will produce a disease which is called anthrax, no other cause can produce it and no matter how much the lesions may vary in different individuals if they are produced by this species of bacteria the disease is anthrax. It is clear, therefore, that there is no hard and fast line between a simple (single) wound infection and a recognized 
infectious or epizoötic disease except in the nature of the invading organism. The lesions may vary and usually they do especially in different species of animals. If a man receives accidentally a cut from a knife with which he is making a post mortem on an animal dead from anthrax, the lesion is liable to be restricted to the point of inoculation, and while it is anthrax (malignant pustule) it would very often be recognized as a simple wound infection. If this accidental inoculation should occur in a guinea pig, the disease would not be recognized as a local lesion; but the animal would most likely die of septicaemia (\$2).

As a class, the lesions known clinically as wound infections are differentiated from the specific diseases in a number of ways. The bacteria commonly found in wound infections do not produce the epizoötic diseases, although there are notable exceptions. Again, there is usually a difference in the mode of infection. The virus of the epizoötic disease is ordinarily introduced through the digestive or respiratory tract or by means of insects, while in wound infection the virus is introduced, as the term implies, through the injured integument or mucosa.

$\$$ 14. The differential characters of a specific infectious or epizoötic disease. It is very important not to mistake for an infectious disease some form of body disturbance due to a local cause or condition. Animals often suffer from improper food and the conditions of life under which they are compelled to live. It frequently happens that as all of the animals in a given herd are subjected to like conditions, a number of them, perhaps all, will manifest very similar symptoms and more or less of them die. Such an occurrence often gives rise to the supposition that the cause of death is some form of infection. Deaths from suclı causes or under such conditions, should be carefully distinguished from an epizoötic. In differentiating a non-infectious disorder from a specific disease, it is important and usually sufficient to take into account the appended characteristics of an infectious disease.

(I) An infectious disease is caused by a specific agent. This necessitates as the first requisite an exposure to and an infection with the specific organism or etiological factor. 
(2) The infection must be followed by a certain period of incubation before the development of symptoms. This is the time necessary for the invading microorganism to become established in the body and to bring about the first symptoms of the disease. The incubation period varies in different diseases and to a certain degree in the same disease according to the mode of infection and the resistance of the individual. Usually the incubation period of a given disease is practically the same for all individuals of the same species when subjected to the same mode of infection. Exceptions, however, are not rare.

(3) The course of an infectious disease is usually nearly the same in animals suffering in the same outbreak especially when they were infected at or about the same time. It is more common for only a few individuals in a herd to be infected in the beginning and from these first cases other animals contract the disease. In many epizoötics, the disease appears in an acute form in the first animals attacked while those infected later in the course of the outbreak suffer from a chronic form of the disease. In other outbreaks, the first cases are chronic in nature and the later ones acute.

(4) In animals, as in man, nost of the infectious diseases are self limiting, but, as a rule, the percentage of fatal cases is much larger among animals than in the human species. The period of convalescence is not so well marked in the lower species as in man. It frequently happens that the course of the disease is so changed that an acute case which appears to recover, or at least to pass into the stage of convalescence, becomes chronic or subchronic in nature and eventually terminates in death. The lateness in the development of the modified lesions often causes the nature of the terminal disease to be unrecognized.

(5) Finally, it is necessary in making a positive diognosis to find the specific organism, or to prove the transmissibility of the malady from the sick or dead to healthy animals. The extent of the spread of the virus of the disease, through the available channels for its dissemination, will also aid in determining the infectious or non-infectious nature of the disease in an outbreak among animals. 
\$15. Cause for the variations in the course of an infectious disease. It is a recognized fact that there is much variation in the course of infectious diseases in different epizoötics and often marked individual variations occur in the same outbreak. In explaining this interesting phenomenon, it is important to take into account the question of individual resistance or immunity-partial or more complete. It was found in case of certain diseases that when an individual is partially immunized and then infected that the lesions were very much modified. The teachings of a specific etiology pointed to this phenomenon as a result of certain biological or vital differences existing either in the parasite or in the host, possibly in both. The results of the investigations already made along this line, suggest a probable explanation, that the course of the disease varies on the one side with the resistance of the host and on the other with the degree of virulence of the infecting microorganism. This has been expressed in the formula

$$
d=\frac{v}{r}
$$

in which $d=$ the disease, $v=$ the rirulence of the infecting organisin, and $r=$ the resistance of the host or the individual attacked. As $\approx$ or $r$ change the disease is modified. For example, rabbits that are partially immunized against swine plague bacteria, when inoculated with a virulent culture of that organism, will live for several days and perhaps for weeks and then die of peritonitis, pleuritis or extensive pus formations (Fig. 3) instead of perishing within twenty-four hours with septicaemia as they would if they had not been protected against this organism. In chronic cases of swine plague, as found in certain outbreaks, the bacteria are often attenuated so that when inoculated into susceptible rabbits the end is the same as when the rabbits protected by partial immunization were inoculated with virulent cultures. The above simple formula which was worked out and demonstrated for certain swine diseases seems to apply to infectious diseases generally.

\$ 16 . Grouping of the specific infectious diseases. It will be found in the study of the morbid anatomy of the 


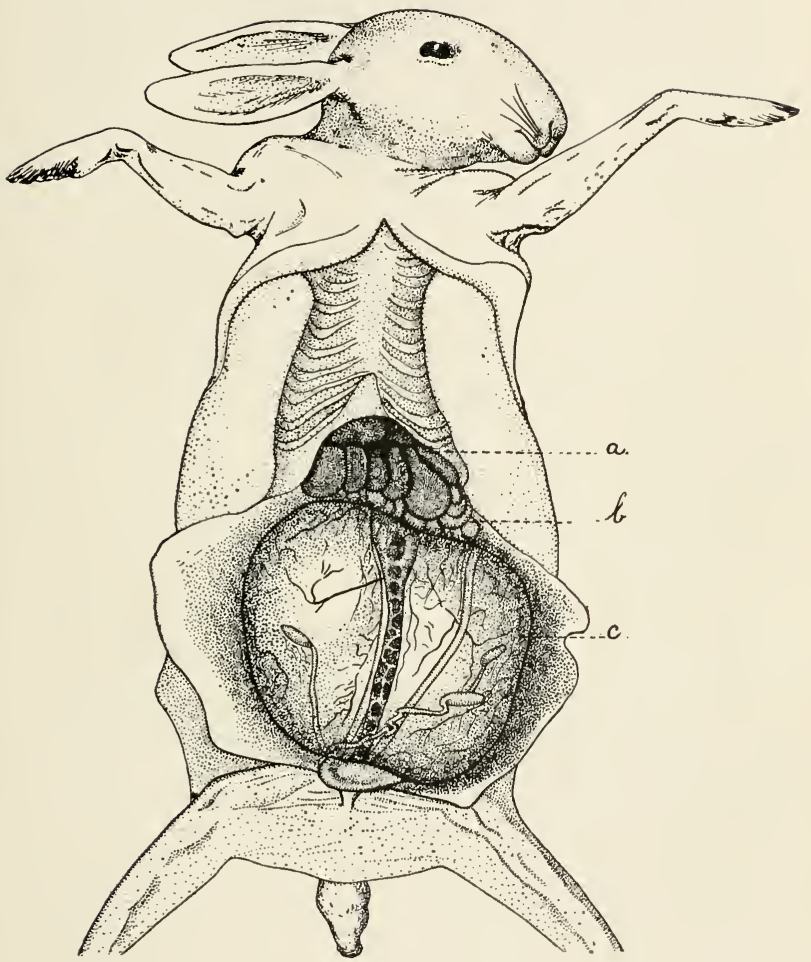

FIG. 3. Large abscess in partially immunized rabbit caused by swineplague bacteria. This rabbit lived 371 days after its inoculation with virulent swine plague bacteria. At death its weight was $1980 \mathrm{grams}$, the tumor itself weighing goo grams. The abscess formed dorsad of the peritoneum. (a) Caecum, (b) intestines, $(c)$ abscess. The control rabbit of same weight and age died of septicaemia in 16 hours. 
various specific maladies that the lesions in a given disease vary in different species and in individuals of the same species to a marked degree. This fact precludes the possibility of classifying or arranging them after their morbid anatomy, if the idea of a specific etiology is to be adhered to. If the infectious diseases are to be considered as parasitisms, as they appear to be, the only logical method of classifying them according to the writer's opinion, is the one suggested by their etiology, namely, that they shall be placed in groups according to their cause. Thus a single lesion found in the glands of the head, in the lungs, in the liver, in the mesenteric glands, in the skin, in the joints, or in the generative organs would be called tubercular if the bacteria of tuberculosis could be demonstrated to be its cause. The same conclusion would be maintained regardless of the character of the lesion, whether cellular, purulent, caseous or calcareous. These facts are enough to suggest that the most direct method of arranging these diseases for purposes of study is in groups composed of like generic etiological factors.

Most of the known specific causes of the infectious diseases of animals are bacteria. It is necessary, therefore, to choose from among the numerous classifications one to be followed in grouping the diseases according to the genera of bacteria producing them. Of the various systems, the one by Migula seems to be the simplest and most natural and consequently it is selected. The only radical difference between it and the others, so far as pathology is concerned, rests in the fact that the old genus Bacterium is revived, but with a new meaning. All rod-shaped, non-motile bacteria are placed in this genus. This causes a change of the generic name from Bacillus to Bacterium of a number of pathogenic bacteria such as those of tuberculosis, anthrax, swine plague and others of less importance.

Following the bacterial diseases are placed those caused by higher fungi.* After the fungous diseases are placed those

*The number of diseases included in this work that are caused by fungi, protozoa and higher animal parasites are so few that they have been grouped under these more general headings rather than separated into groups of the same generic origin. 
due to protozoa. The apparently specific diseases for which an etiological factor has not been demonstrated are grouped together after those of recognized cause. Finally, a few affections resulting from animal parasites are appended for aid in differentiation from infectious diseases that they somewhat resemble in their lesions.

$\$$ I7. Migula's classification of bacteria. The genera are included of the first three families only.

FAMILIES.

I. Cells globose in a free state, not elongating in any direction before division into I, 2 , or 3 planes_............ I. Coccacere.

II. Cells cylindrical, longer or shorter, and only dividing in I plane, and elongating to twice the normal length before the division.

(I) Cells straight, rod-shaped, without sheath, non-motile, or motile by means of flagella

2. Bacteriaceæ.

(2) Cells crooked, without sheath. 3. Spirillaceæ.

(3) Cells enclosed in a sheath .... 4. Chlamydobacteriaceæ.

(4) Cells destitute of a sheath, united into threads, motile by means of an undulating membrane

- 5. Beggiatoaceæ.

GENERA.

I. Coccacece.

Cells without organs of motion.

a. Division in I plane_........ I. Streptococcus.

b. Division in 2 planes_..... 2. Micrococcus.

c. Division in 3 planes_...... 3. Sarcina.

Cells with organs of motion.

a. Division in 2 planes_....- 4. Planococcus.

b. Division in 3 planes_..... 5. Planosarcina. 
2. Bacteriacea.

Cells without organs of motion ..... I. Bacterium.

Cells with organs of motion (flagella).

a. Flagella distributed over the whole body 2. Bacillus.

b. Flagella polar 3. Pseudomonas.

\section{Spirillacea.}

Cells rigid, not snakelike or flexuous.

a. Cells without organs of motion_.................. I. Spirosoma.

b. Cells with organs of motion (flagella).

I. Cells with one, very rarely 2-3 polar flagella 2. Microspira.

2. Cells of polar flagella, in tufts of from $5^{-20}-3$. Spirillum.

\section{REFERENCES.}

1. GAY. A bacteriological study of fistulous withers, botryomycosis and infected wounds in the horse. Am. Vet. Reviez. Vol. XXIV. ( 1901 ) p. 877 .

2. LuCET. Annals de l'Institut Pasteur. Vol. VI ( I893) p. 324.

3. Migula. System der Bacterien. 1897.

4. MOORE. Suppurative cellulitis in the limbs of cattle due to streptococcus infection. Am. Vet. Review. June, 1898 .

5. MOORE. Observations concerning the significance of streptococci in comparative pathology. Am. l'et. Revnew. Jan.-Mar., 1900.

6. MOORE. Preliminary observations on skin disinfection and wound infection. Am. Vet. Review. Vol. XXV. (I9OI) p. 510.

7. NocaRD. A New Pasteurellose: White scours and lung disease of calves in Ireland. Am. Vet. Review. Vol. XXV (1901) p. 326.

S. SMITH AND MOORE. On the variability of infectious diseases as illustrated by hog cholera and swine plague. Bulletin No. 6. U.S. Bureau of Animal Industry. I894. p. 81.

9. WARD. The persistence of bacteria in the milk ducts of the cows' udder. Journal of Applied Microscopy. Vol. I. (r898) p. 205.

10. WARD. The invasion of the udder by bacteria. Bulletın No. 178 Cornell Univ. Agric Exp. Station. 1900.

Ir. Weich. General bacteriology of surgical infections. Dennis, System of Surgery. Vol. I. p. 249. 


\section{CHAPTER II}

DISEASES DUE TO STREPTOCOCCI.

\$ 18. General discussion of streptococci. The confusion which exists concerning species in this group of bacteria and the variety of antistreptococcic serums on the market, renders a summary of the present knowledge concerning this group of bacteria somewhat desirable. The genus Streptococcus is based, according to Migula, on its method of reproduction or division. Streptococci are spherical bacteria which divide in one plane. The segments do not separate but are held together in short or longer chains, although the divisions seem to be complete. Just how the segments are held together is not fully determined. According to older and more commonly encountered classifications, a streptococcus is simply a number of micrococci (spherical bacteria) united in the form of a chain. In some of the supposedly different species the segments are oblong and vary in size. Frequently, however, the segments vary in both size and form in the same chain.

The more usually observed cultural characters and biochemic properties of different streptococci are quite similar, although it is difficult to obtain two cultures that will exactly agree in all of their manifestations when grown on a large number of media. Their disease-producing powers, however, vary within wide limits. While variations in the physiological properties and pathogenesis are true for different cultures (species?), it has been found that there is a possibility of much variation in the subcultures of the same species. As with certain other bacteria, their virulence is the first to suffer change. In differentiating species, therefore, the fact must not be overlooked, that the existing characters and properties possessed by the streptococcus in hand, may have been more 
or less influenced by its conditions of life. When, for example, two streptococci appear to be identical under the majority of tests, a slight deviation in a single property cannot be considered of great differential value especially if this particular manifestation is among those most subject to change. A fundamental difficulty in differentiating species among streptococci seems to be a lack of information concerning the possible variations brought about by different environments. The further difficulty of identifying any of the very large number of forms which have been assigned specific names is due to the brevity of their description and the failure of the author to mention any character or property, or combination of the same, which would distin-

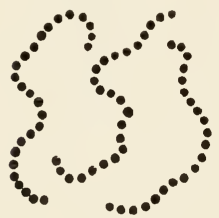

1.

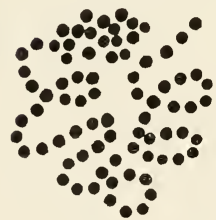

4.

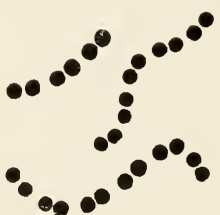

2.

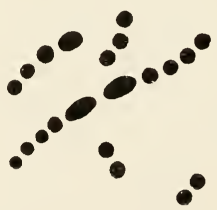

s.

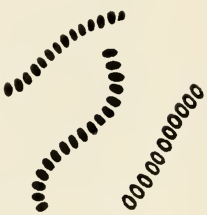

3

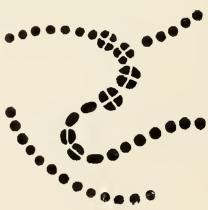

6.

FIG. 4. Six forms of streptococci. I. Long chains consisting of small segments arranged with equal spaces between them. 2. Long and shorter chains in which the segments are arranged in pairs. The size of the individual segments is considerably larger than those in the long chains. 3. Short and longer chains where the scgments are oval with the long diameter perpendicular to the long axis of the chain. 4. Long interlacing chains. 5. Short and longer chains with one or more segments very much larger than the others. 6. Chains showing divisions in two planes. This form of division has been observed in a few cases. The dividing in two planes is an exception which is not satisfactorily explained $\times$ about 1000 . 
guish it from others. However, such deficiencies cannot well be avoided in the time of rapid accumulation of observations and the evolution of methods.

$\$$ 19. Classification of streptococci. A few investiyators have tried to eliminate the confusion concerning species by classifying streptococci according to distinct morphologic characters and pathogenic properties. Of these classifications the following may be mentioned :

I. The classification of von Lingelsheim. This author divides all streptococci into two groups, or species, namely :-

(a) Streptococcus brevis - which is non-pathogenic.

(b) Streptococcus longus-which is pathogenic.

This is a combination of pathogenesis and morphology which the author thought applicable to the entire genus. He worked very largely, however, with the streptococci from the human mouth and throat.

II. The classification of Kurth. Kurth worked largely with the streptococci from cases of scarlatina. His system is practically the same as that of von Lingelsheim, with the exception that he does not include pathogenesis as necessarily belonging to either group. The divisions are as follows :

(a) Streptococcus rigidi-Streptococci growing in short chains, and which impart a uniform turbidity to bouillon.

(b) Streptococcus flexuosi-Streptococci which grow in long interlacing chains which form flocculi in bouillon leaving the liquid clear.

III. The classification of Pasquale. Pasquale worked with thirty-three streptococci, including nearly all of the then known species. His work was quite exhaustive, but he had to deal with cultures of various generations. He divides them into four groups, as follows :

(a) Short saphrophytic streptococci.

(b) Long non-virulent streptococci.

(c) Long pathogenic streptococci.

(d) Short highly infectious streptococci.

Group $(d)$ pertains largely to bacteria which are no longer recognized as streptococci, for example, the diplococcus (Micrococcus lanceolatus) of pneumonia. It is now known that strep- 
tococci which grow in short chains are often virulent. This is especially true of the pyogenic forms.

The study of streptococci from various sources, more especially from tissues of diseased animals, suggests the desirability of delaying a further classification until more definite data are obtained concerning the natural history, not only of these, but also of the species normally present on the mucous membranes of animals, and in nature generally. The specific name is, pathologically or even biologically speaking, of little moment unless we can attach a certain definite meaning to it concerning the morphologic characters, cultural manifestations and the degree of disease-producing power possessed by the organism designated. In a group of twenty-eight streptococci previously studied, the writer found the pathogenic forms, $i . e$, those able to produce disease in rabbits, guinea pigs, or mice, about equally divided between the long and the short chains. Of the twenty-eight, nine possessed a certain amount of virulence for one or more of these animals.

20. Distribution of streptococci in nature. The fact has been pointed out in many publications that streptococci are quite widely distributed in nature. The results of the bacteriologic examinations of normal mucous membranes show that they are frequently included in the bacterial flora of the month, throat, nares, intestines, vagina, and in a few cases they have been found in the bronchioles of the horse and rabbit. They are also present in greater or less numbers on the skin, especially in the deeper layers, presumably in the ducts of the sweat and sebaceous glands and along the hair shafts and follicles. They exist in soil and in water, and occasionally these forms are quite as delicate in their morphology and equally as sensitive to the influence of environment as those isolated from diseased animal tissues. In view of this wide distribution, the presence of a streptococcus in any abnormal condition cannot be considered necessarily a specific infection from a previous case of the same kind. In many affections where the specific organism has been demonstrated, such for example as diphtheria, tuberculosis, and hog cholera, streptococci frequently appear in the lesions. In these cases, they are 
considered as accidental or secondary invaders, although in some of these maladies, such as tuberculosis, they are believed to be of more or less secondary importance. When, however, the specific cause of the disease is not positively known, and streptococci which possess certain pathogenic powers for experimental animals are constantly present and seem to stand in a causal relation to the disease, the pathologist is confronted with a puzzling problem in trying to determine the source and the etiological importance of the organism in hand. In cases of infection leading at once to septicaemia, peritonitis or suppuration, the explanation is more simple than in the epizoötic diseases, such as Brustseuche, where the constant presence of streptococci in the lesions can be quite as easily explained on the ground of their invasion of the parts affected from a normal habitat as on the hypothesis of a specific infection. It is in these instances that we are seeking for the crucial test.

We have found in a few test experiments that when certain of the delicate streptococci which exist (are found) in external nature (soil or water) are introduced within the tissues of certain animals they become, by reason of their activities, a source of irritation which causes local tissue disturbances. In a few cases they have produced septicæmia with fatal results.

In cases of infection resulting in septicæmia, or in those where the disease is more localized, as in strangles or mastitis, and possibly in others where the affection spreads more or less rapidly, we cannot well escape from the feeling that the streptococci, present in such large numbers, must either stand in a causal relation to the disease or be accounted for by their rapid proliferation in native soil made favorable for their excessive increase by the conditions produced by the true etiological factors. Their natural distribution is so wide, and their virulence so capricious that a secondary invasion, which seems always to be possible, renders the fixing of etiological responsibility upon a streptococcus isolated from any diseased tissue a somewhat difficult task. The problems in this connection which concern us most and which need more extended investigation pertain ( $\mathrm{I})$ to the determination of the parasitic possibilities of streptococci existing in nature $i . e$, those ordinarily considered as sap- 
rophytes and (2) to the distinction, if it exists, between streptococci which are able to produce local inflammatory processes leading to suppuration and those which produce highly infective and rapidly spreading diseases, such as ery sipelas and strangles.

In view of the confusion respecting species in this genus, the identity of streptococci isolated from the lesions in the various diseases which have been attributed to streptococci is, at the present time, a matter of some uncertainty. There is also considerable skepticism concerning the primary etiological significance of the streptococci in a number of diseases in which ther have, heretofore, been assigned as the cause. Recent investigations, especially those of Lignières, tend to the conclusion that they are often secondary invaders in certain of these diseases. Petruschky has pointed out analogous cases in human infections in showing that streptococci play an important rôle as secondary invaders in human diphtheria, scarlatina and tuberculosis.

In the absence of verified results to prove the non-specific relation of streptococci to the diseases which have with reasonable certainty been attributed to the activities of this genus of bacteria, these affections are tentatively included among the specific streptococci maladies. It is very important, especially when the use of antistreptococcic serums are in question, to take into account the apparently large number of forms, or species, commonly included in the general statement of a streptococcus disease or infection. In I 897 , Van de Velde, in a very exhaustive series of experiments, showed that one streptococcus antitoxin will not immunize against another save to a very slight degree.

REFERENCES.

1. KLEIN. Seventeenth Annual Report of the Local Government board. Supplement containing report of Medical Officers. London. I887. p 256.

2. KURTH. Arbeiten a.d. Kaiserlichen Gesundheitsamte, Bd. VII, ( I 89 I), S. 389.

3. MOORE. Bulletin No. 3. U. S. Bureau of Animal Indusiry. Washington, D. C. IS93. p. 9.

4. PASQUal. Beiträge zur path. Anat. u. zurallgemeinen Pathologie, Bd. XII (IS93). S. 433.

5. PetruschKy. Zeitschrift f. Hygiene. Bd. XVII. S. 59.

6. VON LINGEISHEIM. Zeitschrift f. Hygiene, Bd. X. I89I, S. 33I.

7. Welch. The Am. Jour. of the Med. Sciences. Vol. CII. (IS9I). p. 439 . 


\section{STRANGLES}

$\$ 2$. Characterization.--Strangles is an infectious disease of horses, asses and their hybrids occurring sporadically and in epizoötics. It is characterized principally by a fever, followed by an acute catarrh of the mucosa of the upper air passages especially of the nares, and a suppurative inflammation of the lymph glands of the submaxillary and pharyngeal regions. The lesions, however, are not restricted to these parts. It is a disease of young animals.

$\$ 22$. History. Strangles was among the first equine diseases to be recognized. In 1664, Solleysel gives an account of it and points to the fact that it had been known for a long time. Its infectious (contagious) nature was determined experimentally in I 790 by Lafosse and since that time by a number of other investigators. In 1873, Rivolta found in the pus of the abscesses a micrococcus which appeared in chains of from three to five segments. Baruchello, in I887, described as its cause an organism, which he designated as Bacillus adenitis equi. Strangles has been tlought by some, to be identical with scrofula and measles. Sacco and Nasbot considered it as horse pox. Viborg and Toggia and more recently Nasbot advocated the inoculation of horses with the lymph of horse pox as a prophylactic measure against strangles. Delamotte demonstrated that this procedure was of little or no preventive value. The supposed specific cause (Streptococcus equi) of strangles was described first by Schütz and later by Sand and Jensen in the same year ( 1888 ). The discovery has been confirmed by Poels, Lupka, and others. More recently Lignièries has discovered a "coccobacillus"' which he believes to be the primary cause. He considers the streptococcus of Schütz as a secondary invader of no specific value.

\$23. Geographical distribution. Strangles is a wide spread disease among horses. It appears to stand in equine pathology very much as measles do in human medicine-a disease of early life and consequently more prevalent where there are more young. It seems to exist in all countries where the horse kind are raised and to be more prevalent in breeding districts than elsewhere. 
$\S 24$. Etiology. Strangles is supposed to be caused by Streptococcus equi, first described by Schütz in 1888 . With pure cultures of this organism Schütz was able to produce the disease in healthy horses. It is fatal to mice, a maximum virulent virus destroying life in three days.

Lignières, who had exceptionally good opportunities in I $895-96$ for studying the disease, found and identified the streptococcus of Schütz. However, he concluded from his investigations that the cause of the disease was a coccobacillus which he isolated with some difficulty. He found that this organism soon escaped from the tissues but that it "places the animal organism in an extraordinary state of susceptility with regard to extraneous bacteria, notably streptococci." On this ground, he explains the almost constant presence of streptococci in the lesions of this disease. The results need to be confirmed.

\$ 25. Symptoms. The first indication of this disease is a rise of temperature. There is loss of appetite, depression, and often great weakness. The general symptoms may continue for a few days before the localization of the lesions is apparent. The first local manifestation consists usually in a catarrh of the nasal mucosa or swelling of the sub-maxillary and pharyngeal lymphatic glands. The nasal discharge is at first serous and somewhat viscid, but in from 3 to 5 days it becomes purulent and of a yellowish green color. The catarrhal condition may exist in one or both nostrils. It may extend into the pharynx, larynx, trachea and even to the bronchi. In most cases, swelling of the sub-maxillary glands appears concurrently with the purulent nasal catarrh. The swelling is hot and painful when pressed. The spreading of the inflammation to the connective tissue which surrounds the glands, and the stasis of the lymph in the efferent lymph vessels, often cause the development, from the sub-maxillary lymph glands, of very extensive swellings that may occupy the entire intermaxillary space, and may spread even to the outer side of the maxilla. In most cases abscesses form.

In exceptional cases, strangles may present catarrhal symptoms without suppuration of the lymph glands. Jensen 
states that it may first assume the form of pharyngitis, purulent pneumonia, and pleuritis without any well-marked morbid affection of the lymph glands. The urine generally remains alkaline; it frequently contains a considerable quantity of albumen.

At times, strangles is accompanied by a cutaneous exanthema which takes the form of an eruption of wheals, nodules, vesicles, and even pustules may appear, chiefly on the sides of the neck, shoulders, and sides of the chest. These exanthemata are characterized by their sudden appearance, and often by their equally rapid disappearance. An eruption of vesicles may break out on the nasal mucous membrane. The contents of the vesicles is at first limpid, but later it becomes purulent. Rabe states that the streptococcus of strangles can produce ulcers on the nasal mucous membrane.

$\$ 26$. Morbid anatomy. The lesions in strangles are interesting from the fact that in the beginning the disease is general but later in its course it becomes a series, exceedingly variable in different individuals, of localized morbid foci. The lymphatic glands seem to suffer most, although any organ may be involved. As indicated by the symptoms, the lesions in most cases are characterized by an acute inflammatory process followed by suppuration.

The glandular swellings about the head usually terminate in suppuration, the pus discharging either externally or into the oral cavity. In other cases, the pus undergoes caseation. Frequently the inflamed glands become confluent, resulting in a single large abscess. Small abscesses may occur under the pharyngeal mucosa.

The inflammation may extend to the superficial lymph vessels of the skin, especially of the head, resulting in the formation of a large number of small abscesses. This may be followed by a diffuse phlegmonous swelling of the parts. Metastatic abscesses are liable to occur in a great variety of organs. The metastasis seems to take place through both lymph and blood ressels although the lymphatic glands are most often affected. Suppurating foci have been described in nearly every lymphatic gland in the body. The discharge of 
pus from the bronchial, mesentric or other glands, within or adjacent to the pleura or peritoneal cavities, may give rise to a fatal pleuritis or peritonitis. There seems to be no part of the body free from possible suppurative lesions as a result of metastasis.

Strangles may become chronic, especially when the nasal catarrh extends into the sinuses of the head, in the guttural pouches, or pharyngeal cavity. In these cases the animal becomes emaciated. The lesions in these cases resemble somewhat those of chronic glanders.

Death from strangles is caused usually by either septicaemia, pyaenia, pleuritis, peritonitis or suppurating (metastatic) pneumonia.

Many complications are liable to arise. Mixed infections or secondary diseased conditions often occur. The prognosis, however, is favorable. The statistics collected from the Euroropean armies show a mortality not exceeding three per cent.

$\$ 27$. Differential diagnosis. Strangles is to be differentiated from:

(I) Purulent nasal catarrh. In this affection, there is rarely suppurating submaxillary glands although occasionally these glands may be swollen.

(2) From glanders. In glanders, the tissue changes are more persistent and the skin lesions, if they exist, do not heal as rapidly as in strangles. In chronic cases, the diagnosis is quite difficult. Here animal inoculations must be resorted to. Mice inoculated subcutaneously with the nasal discharge succumb to the streptococcus of strangles but they are resistant to the bacterium of gianders. Guinea pigs inoculated in a like manner will, in case of glanders, develop that disease $(\$$ 105) from the lesions of which pure cultures of Bacterium mallei may be obtained.

(3) From parotitis. In this affection the swelling is localized and suppuration does not often occur.

(4) Abscesses due to pyogenic bacteria. The cases are rare where there would be any question as to diagnosis. The bacteriological examination including the inoculation of animals would give positive aid unless the pyogenic organism hap- 
pened to be a virulent streptococcus in which case a differentiation might be impossible.

\section{REFERENCES.}

I. Lignitires. The etiology of equine influenza or infectious pneumonia. The Jour. of Comp. path. and Therapeutics Vol. XI (1898) p. 312. Translated from Recueil de Med. Vèt. Vol. IV. (I897).

2. Poles Die Mikrokokken der Druse des Pferdes. Fort. der. Med. Bd VI ( 1888 ) S. 4.

3. REEKS. Intracranial strangles, abscess in a mare. The Jour. of Comp. path. and Therap. Vol XII (1899) p I78.

4. Sand and Jensen. Die Aetiologie der Druse. Deutsche Zeit. fur. Thier Med. Bd. XIII (1888) S. 437.

5. Sснӥтz. Der Streptococcus der Druse der Pferdes. Arch. fur. Thierheilkunde Bd. XIV (1S88) S. 172.

\section{EQUINE CONTAGIOUS PLEURO-PNEUMONIA.}

\$28. Characterization. The disease known as contagious pneumonia or contagious pleuro-pneumonia in the horse is characterized by a high temperature, rapid pulse, but occasionally without definite lung disturbances. Like strangles, both the symptoms and the lesions vary to such a degree that it is difficult to single out diagnostic features. This disease is known in France as pneumoenteritis and in Germany as Brustseuche.

$\$ 29$. History. In earlier times, influenza and contagious pleuro-pneumonia of the horse were not distinguished as separate diseases. Falke differentiated the disease formerly known as influenza into contagious pleuro-pneumonia and influenza. Since his time they have been recognized as distinct diseases.

\$3. Geographical distribution. Contagious pneumonia, like strangles, is widely distributed. It appears in epizoötic form although in certain places it is reported to be almost constantly present. It prevails most extensively where large numbers of horses are congregated. It has frequently been reported as the cause of much trouble among the horses in the European armies. In the eastern part of the United States, it appears from time to time in more or less serious epizoötics. 
It is quite common among horses shipped from the West. In these cases, it is designated as "western" or "stable" fever.

$\$ 3$ r. Etiology. There is some question concerning the specific cause of this disease. A large number of suspected microorganisms have been isolated and described, but the Streptococcus of Schütz seems to be the only one with which the disease has been produced experimentally.

In 1887 , Schütz published the results of his investigations into the cause of Brustseuche. He described an organism which appeared as a diplococcus in tissues, but in bouillion cultures it grew in flocculi. From the description, it appears that this organism was a streptococcus notwithstanding the fact that in the tissues it appeared more often as a diplococcus. In cultures, he speaks of it as chains growing in masses. He mentions a capsule, but in the cases described it does not seem to be invariably present and it is not mentioned in preparations made from cultures. Chantemesse and Delamotte, Galtier and Violet, and Cadéac found streptococci in the lesions of animals suffering from this disease. Although differences seem to exist in the streptococci isolated and studied by these investigators, there is a striking similarity between them. It is not at all unlikely that difference in methods may explain the variations mentioned.

According to Schïtz, cultures inoculated into horses produce the disease when injected directly into the lungs by means of a hypodermic syringe. The resulting contagious pleuro-pneumonia exhibts the same symptoms and runs a like course to those observed in cases of the disease contracted in the natural or common manner. The essential changes shown on post-mortem examination were multiple gangrenous patches in the lungs with parenchymatous degeneration of the most important organs. The inoculated streptococci were found in the tissues of the artificially produced disease. According to Schütz, the bacteria of contagious pleuro-pneumonia are found most numerously in the lungs or the exudate on the pleurae. They are also met with in the nasal discharge and in expired air (Rust). Nothing positive is known concerning the life history of the streptococci outside the animal body. 
They are supposed not to be able to live longer than six weeks within the animal body; but in certain cases, especially in encysted deposits in the lungs, the virus may remain active for a much longer time.

Baunigarten and Hell oppose the view that Schütz's streptococcus is specific, while Rust aud Fiedler support it. Hell maintains that with our present means of investigation, the bacteria of contagious pleuro-pneunionia cannot be differentiated from the pyogenic streptococci or from the streptococcus of erysipelas. In fact, Hell believes that the streptococcus of Schütz has a pathogenic effect in horses affected with pleuro-pneumonia; but as there is no positive proof of its being specific, he maintains that we are justified in supposing that this ubiquitous micro-organism has only an injurious influence on the course of the disease, contributing to the production of the secondary lesions. Hell further states that protective inoculation with Schütz's bacteria, which at first promised good results, has not proven to be satisfactory. Fiedler, on the other hand, has obtained the same bacteriological results and has arrived at the same conclusions as Schütz. He also states that he has experimentally produced pleuropneumonia in a horse by inoculation of cultivations of these bacteria.

Lignières (See $\$ 24$ ) believes that his cocco-bacillus stands in an etiological relation to this disease and that here as in strangles the streptococcus is a secondary invader.

The writer made a bacteriological examination of the organs from five cases of fatal contagious pueumonia of the horse. In each case, the lungs were more or less hepatized. but the other organs were nearly normal in appearance. Without exception, a streptococcus appeared, usually in pure culture, from the lungs. The inoculated media from the other organs, liver, spleen, and kidney remained clear. The streptococci isolated from the different cases were identical in their morphology and cultural manifestations and pathogenesis. A microscopic study of the lungs from the different horses showed micrococci singly, in pairs and occasionally in short chains. Distinct capsules were not observed. In boullion 
cultures, however, they appeared in long chains, leaving the liquid clear, as described by Schütz.

This streptococcus did not grow in gelatin, or on serum, or on potato. It would not develop in acid media. On agar, the colonies were small and characteristic of streptococci, i. e., with a thickened, convex, grayish center surrounded by a thin, spreading bluish border, nearly equal in width to the diameter of the central portion. It fermented dextrose, lactose and saccharose, with the formation of acids but no gas. Milk remained unchanged in appearance.

In mice and rabbits, it produced a rapidly fatal septicaemia, but guinea-pigs were unaffected. A horse inoculated in the pleural carity with a small quantity of the culture was killed Io days later. At the point of inoculation and extending over an area equal to one-half of the ling, there were strong adhesions between the lung and parietes. The subjacent lung tissue was hepatized. Pure cultures of the streptococcus were obtained from the exudate and from the hepatized lung.

Although a few discrepancies exist between the description of Schüt's organism and this streptococcus, in the more essential features they seem to be identical. The cases were examined before the publication of Lignières' results, and the methods employed did not meet the requirements of those used in isolating his cocco-bacillus. Although a very careful histological study of the pneumonic tissue was made, Lignière's organism was not detected.

$\S 32$. Symptoms. The period of incubation is given as varying from one to fourteen days, but usually from four to ten days elapse from the time of exposure to the development of the first symptoms. 'The symptoms vary to a marked degree. When pneumonia develops early in its course, the disease may appear suddenly; and, in addition to the elevation of temperature, there is cough and difficult breathing. Often the symptoms differ from those of fibrinous pneumonia by the absence of distinct evidences of local lesions which are found in that disease. The first regular sympton is a rapidly increasing temperature frequently accompanied by a chill. The pulse rate is increased. There is general 
depression, usually loss of appetite and muscular weakness: the conjunctivae and other visible mucous membranes become congested. There may be from the beginning marked indications of localized lesions in the lungs, or the general symptoms may continue without evidence of pronounced lung disturbance. The duration of the disease depends almost entirely upon its course. In the more typical cases, the fever lasts from 5 to 8 days. The period of convalesence is much longer lasting from two to three weeks. Nany symptoms may be exhibited corresponding to the variations in the morbid processes. If the heart, digestive tract, liver, kidneys or brain become the localized seat of the disease, symptoms referable to impaired functions of these organs are in evidence. The septicaemic form has been described as being followed by localized suppurative lesions.

\$3. Morbid anatomy. The morbid changes in the tissues and organs vary according to the course of the disease, which is exceedingly irregular. It may exhibit a regular form of lobar inflammation of the lungs or the disease may run an atypical, complicated, acute, chronic, and not infrequently an abortive course. Further, authorities agree that many complications may arise modifying or changing completely the morbid anatomy of the disease from the conditions found in the more typical cases. In the few cases examined post mortem by the writer, the gross lesions were restricted to the lungs. They were either in a state of congestion, or exhibited changes of fibrinous pneumonia in the cephalic (anterior) portions of one or both organs. Pneumonia is the most common localized lesion. Several quite distinct forms of lung disturbances are described.

In the lobular form of pleuro-pneumonia which is stated furnishes the largest number of subjects for a post mortem examination, there is a multiple haemorrhagic, gangrenous pneumonia with secondary pleuritis and possibly parenchymatous degeneration of the vital organs of the body. Areas of the lung tissue of greater or less size are thickened and hepatized. These are located more especially near the base of the lungs and in the lower (ventral) portions. Bright foci 
which are distinctly defined from the neighboring tissues are scattered through the hepatized areas and appear on the surface of sections of the hepatized parts. Usually, several of these foci are present. They vary in size from a millimeter to 20 or more centimeters in diameter. In recent lesions, these areas are very small, of a greyish-red color and surrounded by a grayish zone consisting of leucocytes (limited reactionary inflammation). In more advanced lesions, they become yellowish, necrotic and finally cavities varying from the size of a pea to that of a hen's egg. These cavities are surrounded by a smooth capsule. There are other foci which contain greasy, foetid, watery pus (gangrene of the lungs), by reason of the necrotic parts of the lung undergoing liquefaction in consequence, it is stated, of the admittance of air. The lungs often contain suppurating foci composed of a whitish pus mixed with necrotic lung tissue. It sometimes happens that the foci just described are absent in the lungs, although during life distinct symptoms of such a localized affection may have been present. In these cases, it is assumed that absorption of the necrotic tissue has taken place. The remaining tissue of the lungs is, more or less, hyperaemic and oedematous.

The pleurae show signs of a diffuse, exudative pleuritis, the starting point of which in the large majority of cases, is from necrotic deposits which are situated in the periphery of the lungs. Pleuritis may occur, however, apparently as a primary lesion without the necrotic foci being present. The contents of a necrotic deposit in the lungs rarely breaks through into the pleural cavity. In some cases, the visceral and costal layers of the pleura are congested, diffusely or in spots, and are sprinkled with haemorrhages. Frequently the pleurae are covered with soft red granulations covered with soft, yellowish layers of exudate which are partly membranous and partly coagulated in a reticular manner, and which can usually be easily removed. The pleural cavities generally contain a considerable quantity of fluid. Dieckerhoff states that from 30-40 liters of a serous fluid are occasionally present. The exudate is usually turbid and of an orange, greyish-red, brownish-red, or dirty-greyish color. It is generally mixed with nu- 
merous yellowish colored flakes which form a sediment when the liquid is allowed to stand in a glass. The pleuritic exudate sometimes consists of pure pus and less frequently of blood. The pleuritic exudate when present in large amount compresses the lungs and pushes them away from the thoracic walls. In cases of recovery, the pleuritic exudate may become organized, binding the lungs to the costal walls and diaphragm. Various forms of fibrous, villous growths develop on the pleurae.

The records show that the other organs of the body are usually in a state of parenchymatous inflammation and fatty degeneration. The muscular tissue of the heart is, as a rule, brownish-grey in color, soft and suffers from cloudy swelling. In severe cases, it shows well-marked fatty degeneration, is of a clay color, and is occasionally infiltrated with a large number of small, yellowish-white foci. The liver is enlarged, of a clay color or sometimes icteric, and presents signs of fatty degeneration. The spleen is flaccid, its pulp increased and often sprinkled with haemorrhages. The kidneys may be swollen, friable and sometimes show numerous haemorrhagic foci. The lymph glands, especially the bronchial and mediastinal glands, are enlarged, softened, and exhibit on section a greyishred color. The muscles of the body are soft, and of a yellowish-brown color. Snull haemorrhages under the serous menbranes are frequently reported. Slight endocarditis may occur. The blood is said to suffer less change than any of the solid organs.

The mucous membrane of the stomach and intestines is frequently hyperaemic, swollen, sprinkled with haenorrhages, and sometimes even ulcerated. The bronchial mucous membrane is also swollen and inflamed.

In other cases, the lesions are those of lobar pneumonia, in which the stages of hyperaemic, red hepatization, grey hepatization and resolution (in favorable cases) follow each other in regular order. In a fatal case postmortemed by the writer the right lung was entirely involved, the left one being but slightly hyperaemia. In non-fatal cases, the crisis is reached on the 5 or 6 day, after which resolution begins. 
As already stated the lesions in this disease are so exceedingly variable that, in addition to the more typical pneumonia, almost any modification can be found. The detailed description of many of these variations as described by Dieckerhoff are worthy of careful study.

S 34. Differential diagnosis. This disease is to be differentiated from ( $\mathrm{I}$ ) influenza, with which it is often confused, (2) simple pneumonia following colds, and (3) the result of foreign substances introduced into the bronchi.

\section{REFERENCES.}

I. CADÉAC. Contribution a l'etiologie de la pneumonia contagieuse du cheval. Comp. Rend. de la Soc. de Biol. 1859. p. 316.

2. FLEMING. Infectious pneumonia of the horse. The Veterinary Jour. Vol. XXXIII p. 1.

3. Schütz. Die Ursache der Brustseuche der Pferde. Virchow's Archives. Bd. CVII. S. 356 .

4. Scнütz. Die genuine Lungenentzündung der Pferde. Archiv fur IVissen, u. prak. Thierheilkunde. Bd. VIII.

5. Sifdalgrotzky. Ueber infectiöse Pneumonien bei Pferden. Deutsche Med. Wochenschrift. 1882. S. 668.

6. Wilitams. Contagious pleuro-pneumonia of the horse. Am. Vet. Review. Vol. XVI (1892) p. 301.

\section{APOPLECTIFORM SEPTICAEMIA IN CHICKENS.}

\$ 35. Characterization. A rapidly fatal septicæmia in chickens caused by a streptococcus.

$\$ 36$. History. This disease was recently discovered and described by Nörgaard and Nohler. Although the șrmptoms and lesions given correspond somewhat closely to those mentioned by Mazza and Rabieux, there is a marked difference in the etiological factor. This newly discovered disease is based upon the findings and investigations following a single epizoötic among fowls. Thus far it has not been identified in any other locality.

$\$ 37$. Geographical distribution. The only locality from which it has been described is Loudoun Co., Va.

$\$ 38$. Etiology. This disease is stated to be due to a streptococcus which grows in short or longer chains with segments varying from 0.6 to $0.8 \mu$ in diameter. In some cases 
elongated forms are observed. It is an ærobe, and a facultative anærobe. When cultivated on artificial media it does not liquefy gelatin, it does not change the appearance of milk, but causes slight acidity and thickening of the lower stratum without coagulation of the casein. The reaction of alkaline bouillon is changed to an acid one and it does not give a visible growth on potato. It stains by Gram's and Gram-Weigert's method. In bouillon it grows in somewhat flaky masses while the medium remains clear. It was fatal to fowls, mice, rabbits and swine; guinea pigs, dogs and sheep were not destroyed by inoculation. This organism has not been given a specific name.

\$3. Symptoms. It is not at all uncommon to find the fowls dead and lying under the roosts. Occasionally capons were observed to be sick for from 12 to 24 hours prior to death. In these cases the feathers become ruffled and the fowl showed evidence of extreme depression. The onset of the disease is very sudden and its course a very rapid one, usually terminating in death.

$\S 40$. Morbid anatomy. The authors describe the morbid anatomy as follows: "The spleen is more or less enlarged, showing hyperplasia of the Malphigian corpuscles. The pulp contains numerous areas of extravasated blood. When a stained section is examined by means of a hand lens a number of circular semi-transparent foci, the size of a pin hole, may be noted. These are found on microscopic examination to be centers of necrobiosis, consisting of parenchyma which has undergone coagulation necrosis, and surrounded by a more or less well defined capsule of embryonic and further developed connective tissue cells and filaments.

On microscopic examination, the kidneys show slightly swollen epithelial cells of a beginning parenchymatous degeneration to the well pronounced disintegration of the renal epithelium of acute nephritis. The degree of degeneration depends, as a rule, upon the course of the disease. If a bird succumbs suddenly or in the course of a few hours the morbid changes are either not apparent at all or but slightly pronounced, while, on the other hand, the duration of three or 
animal. On account of its frequent association with hog cholera, it has been thought by some investigators, more especially Billings, Welch and Clements, to be a secondary affection only. In 1895, the writer investigated several outbreaks of this disease in Southern Minnesota where it occurred uncomplicated with hog cholera. More recently two epizoötics of swine plague have been studied in New York State, where 110 evidence was found of its being a secondary infection but where in every particular its independent nature was indicated.

$\$ 45$. Geographical distribution. Swine plague is a wide spread disease in this country. It seems to occur more or less frequently in every state in the Union. It is quite widely distributed in Germany, but to what extent it exists in other countries there is little or no available evidence.

$\$ 46$. Etiology. Swine plague is caused by a non-motile elongated, oval bacterium described by Smith in 1886 . It is identical with the bacillus of Schweineseuche

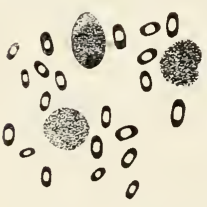
described by Loeffler in i 885 . Hueppe proposed the name Bacterium (Bacillus) septicaemiae hemorrhagicae for this organism.

The bacterium of swine plague and its varieties have not been systematically studied and classified. It is of interest to note,

FIG. 5. Bacteri- however, that the bacteria of rabbit septium of swine plague. caemia, fowl cholera and wildseuche are thought to be identical with it.

The pathogenic organism associated with the lesions in certain forms of broncho-pneumonia in cattle differs very slightly from this. In human pathology, we find a striking resemblance in Micrococcus lanceolatus to the swine-plague bacterium, especially in its manifold and varied pathogenic possibilities and its existence in human saliva.

In grouping these bacteria, the fact should be recognized that experimentally they are not interchangeable in their pathogenesis except for the rabbit. Thus an epizoötic form of fowl cholera has not been produced with the swine plague or rabbit septicaemia organism. Further, it has been shown 
that in the upper air passages of healthy swine, cattle, horses, cats and dogs* there are bacteria not distinguishable in their cultural characters and their effect upon rabbits from the swineplague bacterium. The presence of this organism in the trachea of healthy pigs has been suggested as the source of the cause of sporadic cases of swine plague and it may explain the frequent association of this form of pneumonia with hog cholera. What the conditions are by which these bacteria are enabled to produce disease in their host have not been clearly pointed out.

If the rabbit is taken as the animal on which to test the pathogenesis of the bacteria belonging to the swine-plague group, we find that those from different sources are very similar. In nature, the bacteria of swine plague, rabbit septicæmia, fowl cholera, and those located in the normal upper air passages of the various species of animals mentioned possessed of marked variation in virulence exist, that is, those which will kill a rabbit when inoculated subcutaneously with a pure culture in from 16 to 24 hours to those which require from 3 to ro days, or even weeks, to destroy life. With the variations in the time period, we have corresponding differences in the lesions. The virulent forms produce septicæmia while the attenuated varieties excite a severe purulent infiltration about the place of inoculation and exudates on one or more of the serous membranes. Conversely, it has been shown that rabbits possessed of a certain amount of natural or artificially produced resistance will, when inoculated with a virulent culture, die after the same period of time and with lesions similar to those produced by the attenuated virus in the susceptible rabbit.

The fact that this organism is not frequently described in our works on bacteriology and because of some confusion existing concerning it and the bacillus of hog cholera, it is deemed best to insert a short description of it here.

*The investigations thus far made show these bacteria to be present in $4^{S}$ per cent. of healthy swine, 80 per cent. of cattle, 50 per cent. of sheep, 16 per cent. of horses, 90 per cent. of cats, and 30 per cent. of dogs. 


\section{$\$ 47$. Brief description of the bacterium of swine plague.}

Morphology.-A non-motile, rod-shaped organism varying from o. 8 to 2.0 microns in length and from 0.4 to 1.2 microns in breadth. The ends are oval, and the shorter forms resemble micrococci. The size depends upon the medium and the stage of development of the individual bacteria. A capsule has not been demonstrated, although often there appears to be one in preparations made directly from tissues. It is not observed in cultures. Spores have not been seen. Involution forms are not uncommon in old cultures. They are especially numerous in the organs of a rabbit when it is allowed to lie for some hours after death, before it is examined. It exhibits, when stained in cover-glass preparations made directly from animal tissues, a light center with deeply stained extremities (polar stain). In preparations made directly from cultures this character is much less marked. It stains readily with the basic aniline dyes. It does not retain the coloring matter when stained after Gram's method.

Cultural and biochemic properties.-This organism is less hardy than the bacillus of hog cholera, and on certain of the media used it grows very feebly or not at all. It requires a temperature of about $37^{\circ}$ C. although it develops very slowly at the room temperature.

Agar.-The growth on this medium is not vigorous. It is of a neutral grayish color, with a glistening, moist appearing surface. It is slightly viscid and adheres to the agar surface. Isolated colonies vary from I to $2 \mathrm{~mm}$. in dianieter, nearly round, convex, with smooth and sharply-defined margins. The condensation water becomes faintly clouded with a grayish sediment which becones viscid. Within the agar the colonies appear as minute grayish dots. In agar, especially in plates (Petri dishes), it enits a peculiar, disagreeable, pungent odor.

Gelatin.-Ordinarily it does not grow in gelatin. (Dr. Theobald Smith found that certain cultures grew in this medium.)

Potato.-It does not grow on potato.

Bouillon.-Alkaline peptonized bouillon becomes uniformly clouded in 24 hours when kept at a temperature of $36^{\circ} \mathrm{C}$. Occasionally cultures are obtained in which the growth appears in the form of flocculent masses, but usually after a few generations these disappear and the liquid becomes uniformly cloudy. If the bouillon contains any dextrose or muscle sugar, its reaction becomes acid in 24 to $4 \mathrm{~s}$ hours, owing to the fermentation of the carbohydrate. With the virulent cultures the liquid clears within a few days. The small amount of grayish sediment becomes viscid after some days, and upon agitation it is forced up, appearing as a somewhat twisted tenacious cone, with its apex at or near the surface of the liquid. Frequently a thin, grayish, somewhat viscid band composed of bacteria is found on the sides of the tube at the surface of the liquid. It will not grow in acid bouillon. If the bouillon 
contains from I to 2 per cent. glucose, the growth is slightly more vigorous.

Effecl on sugars. - In the fermentation tube, alkaline bouillon containing sugars become uniformly clouded in both branches. Gas is not proiluced. In bouillon containing dextrose and saccharose the reaction becomes strongly acid in 24 hours, but the reaction of alkaline bouillon containing lactose is not changed.

Milk.-Milk inoculated with this organism remains unchanged in appearance for several weeks. When boiled, after this period, the casein is not coagulated.

Indol.-This organism grows feebly in Dunham's solution; some cultures have given a decided indol reaction, but others have not. The production of indol is reported to be one of the properties of the German swine plague. Smith* obtained only a trace of indol in one out of four cultures of swine-plague bacteria.

Phenol. - This was found by Lewandowski's $\dagger$ method in all of the cultures tested by Smith. I have failed to obtain the reaction in a few cultures, but usually it appears.

Thermal death point.-This organism is destroyed in bouillon at $5^{5^{\circ}} \mathrm{C}$. in ten minutes. A temperature of $56^{\circ} \mathrm{C}$. for this tine did not destroy its vitality.

Effect of drying.-These bacteria can not stand drying. The bacteriam in a drop of bouillon dried on a cover-glass and kept at the room temperature are destroyed in 24 to 36 hours. In similar preparations made from agar cultures they resist drying from five to eight days. The difference in the time between the two cultures is probably due to the thicker layer in case of the agar preparations.

Persistence of vitality in water and soil.-Experiments to determine the length of time this organism will live in water and in the soil show that it is destroyed in water in test tubes in from nine to eleven days. In the soil it was not found after eight days. Dr. Smith states that it is destroyed in the soil after four days.

Power to resist disinfectants. - The bacterium of swine plague is very sensitive to the action of disinfectants. A large number of these agents have been tested. The following are among the more important :

Commercial sulphuric acid, 1/8 per cent. kills in 30 minutes.

Commercial sulphuric acid, $1+$ per cent. kills in 10 minutes.

Lime, lime water kills in $\mathrm{r}$ minute.

Lime, o.or 5 per cent. kills in 30 minutes.

Carbolic acid, $1 / 2$ per cent. kills in 60 minutes.

Carbolic acid, I per cent. kills in 5 minutes.

Carbolic acid, 2 per cent. kills in I minute.

*Special report on swine plague, I $\$ 91$, p. 89.

†Deutsche ned. Wochenschrift, r 890, s. I1 $\$ 6$. 
Formalin, solution I-2000 kills in 5 minutes.

Trikresol, $1 / 2$ per cent. kills in 5 minutes.

Pathogenesis.-This organism is pathogenic for rabbits, guinea pigs and mice among the smaller experimental animals and for swine. With the virulent form rabbits inoculated either subcutaneously or in the vein with very small, o.oor c. c. doses, die of septicæemia in from 16 to 24 hours. Guinea pigs are slightly less susceptible. When inoculated subcutaneously with 0.1 to 0.2 c. c. of a bouillon culture, they die in from 30 to 72 hours. Mice succumb in about 24 hours when inoculated with a drop of the culture. Pigs inoculated intravenously usually die from acute septicæmia in from 18 to 36 hours. If they live longer there may be decided lung lesions. (See report on swine plague, Smith.)

$\$ 48$. Symptoms. It is frequently difficult to recognize symptoms distinctive of swine plague. The peculiarities of swine render it exceedingly difficult to obtain evidence on physical examination of lung disease. Somretimes this affection runs a very rapid course, the animal dying of septicaemia. Usually it is more protracted, lasting from a few days to a week or longer. Animals affected with the more chronic form where there are lung lesions, eat very little, or refuse food altogether. They cough considerably, especially when forced to run. The back is usually arched and the groins sunken. The whites of the eyes are reddened. The skin over the ventral surface of the body, nose and ears is frequently flushed. The cough, however, is the most reliable indication we have of swine plague; but in some cases of hog cholera the coexistence of broncho-pneumonia also causes the animal to cough when forced to move rapidly.

$\$$ 49. Morbid anatomy. There are many known variations in the appearance of the internal organs of hogs which have died of swine plague. The characteristic lesions are, as previously stated, to be found in the lungs. Frequently the abdominal viscera appear to be normal although a careful examination will usually reveal slight changes. In the lungs, however, the disease is usually obvious.

The variety of lesions produced by the inoculation of swineplague bacteria is not so great as that observed in the naturally contracted disease. While there are outbreaks in which considerable uniformity is observed, there are others in which each animal is a surprise to the pathologist. In general it may 
be stated that the lungs and the digestive tract are the chief seats of the disease, though other organs, notably the lymphatic glands, are secondarily involved. The disease is localized in the lungs and in the digestive tract most likely because the bacteria gain entrance through the respiratory and digestive passages.

The lungs have been found diseased in nearly every outbreak which has been investigated. In some outbreaks the lung lesions predominated and pneumonia was the direct cause of death. In individual cases, pneumonia is absent but pleuritis and interlobular oedema are genearlly present. In a few instances interlobular emphysema of the lungs has been observed. With pneumonia the ventral lobes are first attacked, then the cephalic and azygos, and lastly the principal lobes. This movement of the disease seems to depend on gravity, inasmuch as the diseased parts are marked off from the healthy portion by a nearly horizontal line. In other words, the most dependent portions of the lungs are the ones affected first, and as the disease pro- portion of lung showing infiltration into the interlobugresses upwards only a small portion lar spaces with serum and of the principal lobe directly under the lymph.

back of the animal, remains pervious, provided the life of the animal is maintained up to this point. In nearly all cases of pneumonia in swine the disease involves the regions described which are indicated in the cut by shading. In exceptional cases, where the disease is caused by lung worms or due to embolism, the pueumonia involves portions of the principal lobes not contiguous to the ventral lobes.

Two kinds of pnemmonia are encountered, lobar and catarrhal or broncho-pneumonia. In the former the vesicular portion of the lung substance is chiefly affected; in the latter 
the smaller bronchioles are said to be primarily affected and the vesicular portion or alveoli secondarily. In croupous pneumonia, there is, following the stage of congestion, an emigration of red blood corpuscles, some leucocytes and an exudate of fibrin into the air spaces. These elements are firmly matted together by the coagulating fibrin, making the diseased lung firm to the touch. In broncho-pneumonia the catarrhal condition of the smaller air tubes makes them impervious to air. The lung tissue which they supply is gradually emptied of air and assumes the appearance of red flesh, owing to the collapse of the walls of the alveoli and the distended condition of the capillary network. Subsequently the inflammation extends into the alveoli, which then become distended with cellular masses.

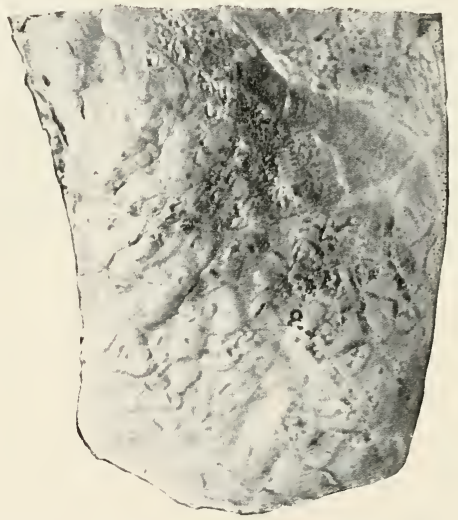

FIG. 7. Portion of lung shozeing emphysema.

The nature of the lung disease will depend more or less upon the mode of entrance of the virus. If it entered only by way of the air tubes it will appear perhaps as a bronchopneumonia. If it enters the lung tissue through the circulation we may have more or less scattered centers of hepatization:"(embolic pneumonia.) If it enters by way of the pleura, 
eased ventral lobe, and that the resting of a lobe against an inflamed serous surface, such as the pericardium, caused a pneumonic infiltration at the point of contact.

The character and seat of the lung lesions are somewhat variable. It is difficult to find two lungs exactly alike so far as gross appearances go. This to be sure may be due largely to the fact that animals die in different stages of the disease. Yet there are differences evidently not dependent on this fact which must be left for special pathological investigation.

In general the cephalic (anterior) half of a swine-plague lung is hepatized, of a dark-red or grayish-red color and firm to the touch. The pleura is more or less thickened and opaque, and possibly covered with easily removable, friable, false membranes. In the more recently affected regions a faint but quite regular delicate mottling with yellow is observed to shine through the pleura when not thickened. These minute hazy, yellowish dots usually occur in groups of four. Occasionally whitish or yellowish patches varying much in size are seen perhaps more frequently in the ventral lobes. These correspond to homogeneous dead masses of lung tissue.

When such lungs are cut open, the section presents much the same appearance, both as regards color, and mottling, as when viewed from the surface, excepting that the details are less distinct. In some cases in the most recently invaded territories in the principal lobe and nearer the dorsum in the other lobes, the dark or grayish-red cut surface shows grayish lines usually arranged in curves and circles. These, so far as determined, represent the cut outlines of the interlobular and peribronchial tissue infiltrated with cells. It has already been stated that these lines may represent the paths along which the swine-plague bacteria invade the lungs from the pleural surface.

The cut ends of the bronchi of the ventral lobes are frequently occluded with thick, whitish pus; in the other lobes a reddish froth is usually present. Rarely they also contain thick glairy mucus in which particles of dry pus and lung worms are imbedded. The contents of the air tubes in the 
ventral lobes may have been derived from the overdistended alveoli, or else a broncho-pnemmonia, may have preceded the swine-plague pneumonia.

In microscopic sections of diseased lung tissue the alveoli and smallest air tubes are found distended with cell masses consisting chiefly of leucocytes. Usually there is very little fibrin and very few red corpuscles in the alveoli, even in cases in which the disease was quite recent. It may be that the stage represented in ordinary croupous pneumonia by the presence of fibrin in connection with the cellular elements is very brief, and that it is speedily replaced by large numbers of leucocytes. The large predominence of these elements in some portions of the lungs, as well as beginning fatty degeneration, is probably the cause of the regular mottling of the lungs, as seen from the surface. The little yellowish hazy dots represent alreoli surrounded by the hyperaemic walls.

The necrotic and caseous changes so frequent in swine plagne are most interesting. The latter are usually quite small and disseminated in large numbers over the diseased lobes. The former represent larger masses from a marble to a horsechestnut in size. They represent tissue which has been destroyed by the rapid multiplication of swine-plague bacteria in particular localities. Hence they are found in all stages of the pneumonia. The large caseous masses may be considered as the result of a slow death of larger areas of lung tissue, due primarily to the gradual orerdistention of the tissue by leucocytes, and hence the gradual cutting off of the blood supply. One is a rapid death due directly to highly virulent bacteria, the other a slow death, or a kind of dry suppuration in the later stages of the pneumonia, characteristic of the pig, and due indirectly to the irritation of perhaps more attenuated races of bacteria. In some cases there are extensive hemorrhages in the interlobular connective tissue. (Fig. 9.)

The inflammation of the pleura frequently extends to the pericardium. This membrane is opaque, thickened, and its ressels distended. It may be glued to the contiguous lobes of the lungs and covered by a false membrane, smooth or roughened, which extend upon the large ressels emerging at its base. 
Disease of the digestive tract in a considerable proportion of animals inoculated with swine-plague cultures consisted in a severe catarrhal inflammation of the lining membrane of the stomach. The hyperæmia was very intense, bordering on hemorrhage. Occasionally the extension of the peritonitis, produced by intra-abdominal inoculation, along the mesentery causes a severe inflammation with exudation on the inucosa of the small intestine. A case is reported where all the Peyer's patches of the small intestine were in a hyperæenic, and partly hemorrhagic condition.

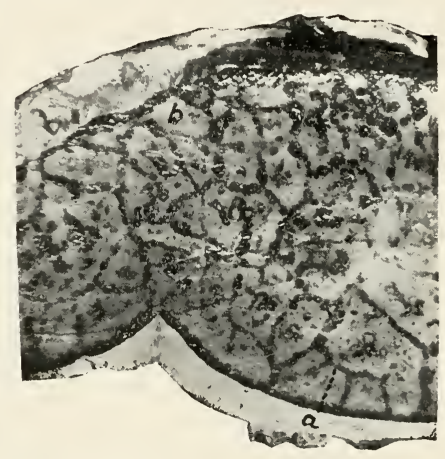

FIG. 9. Hemorrhage into interlobular tissue of a swine-plague lung. - (a) Hemorrhage, (b) hepatized lobules

In the naturally contracted disease extensive hyperaemia of the mucosa of the large intestine bordering on a hemorrhagic condition has been observed. In other cases a peculiar croupous exudation appeared, which seemingly resulted from the effect of swine-plague bacteria in the large intestine.

The production of intestinal disease by swine-plague bacteria may be supposed to go on as follows. The bacteria first attack the lung tissue and there produce more or less hepatization. The blood through the lungs finds its path partly obstructed. This reacts on the blood in the right side of the heart and the venous blood entering it. Hence there may be 
more or less stasis of blood in the portal circulation which in turn impairs the digestive functions of the stomach. The swine-plague bacteria in the lungs in the later stages of the pneumonia may be coughed up in the contents of the bronchial tubes, swallowed and passed through the impaired stomach umharmed into the intestines. The stagnation of the feces in the large intestine furnishes the bacteria an opportunity to cause inflammation with exudation of the nucous membrane. The tendency of swine-plague bacteria to cause fibrinous inflammatory deposits on serous membranes may serve to explain such action on mucous membranes.

There is general congestion with resulting degeneration of the parenchyma of the spleen, kidneys and liver in the acute septicaemic forms of the disease. In these cases the specific bacterium is easily obtained from the abdominal organs. In brief, the lesions of swine plague as they appear in various outbreaks may be summarized in four classes, namely :

I. Acute septicaemia form in which the lesions are characterized by a general hyperaemic condition with possibly hemorrhages on the serous membranes and in the parenchynatous organs. No localized lesions.

2. Cases of pneumonia with or without pleuritis. The other organs remaining normal in appearance.

3. Cases where either in addition to, or possibly in the absence of, the lung lesions there are marked anatomical changes in the mucosa of the digestive tract and possibly in the lymphatic glands.

4. Cases of mixed infection, especially with hog cholera, where in addition to the swine plague lesions which may be more or less modified, there are those, especially of the digestive tract, characteristic of the accompanying disease.

In order to present as clear a picture as possible of the findings in a case of this disease, I have appended the published autopsy notes of one animal. (Smith's report on swine plague, p. 62.)

"Pig died yesterday, put on ice. Weighs about 30 pounds. Skin on ventral aspect of body more or less reddened; over the sternum a few excoriations. The enlarged inguinal glands show as lumps under the 
skin. On section they present a mottled gray and red surface, the red limited chiefly to the cortex. Oedema of the subcutis over right knee.

False membrane covers the left half of the mass of intestines and the spleen; consists of an elastic, rather firm yellowish white layer. Spleen firmly glued to the surrounding intestines, slightly enlarged, dark, softened. Liver firm, cuts with considerable difficulty. Kidneys in condition of parenchymatous degeneration. One hemorrhagic spot in medullary portion of the kidney. Pelvis contains a whitish glairy liquid.

Digestive tract. Two superficial necroses on the inner surface of lower lip in front, one on the upper lip and on edge of tongue near tip. Stomach contains a little deeply bile-stained fluid. Mucosa sprinkled with red spots of a washed-out appearance, most numerous in fundus and near pyloric valve. Hyperæmia of duodenum begins sharply at pyloric valve. From the opening of bile duct a few drops of thick bile can be expressed. Remainder of small intestine not markedly changed. The Peyer's patch in lower ileum has some of its follicles enlarged from which caseous masses can be expressed.

Large intestine contains much sand and gravel. Mucosa of cæcum of a dark slate color. The summit of the folds of a purplish hue. Free edge of valve bordered by a thin slough. On Peyer's patch near valve areas of necrotic tissue of a yellowish color, resting on a firm, yellowishwhite base three-sixteenths of an inch thick. Upper colon has its mucosa of the same dark slate color, merging into a wine red. Two ulcers one-eighth of an inch in diameter observed. In lower colon congestion slight and gradually disappearing towards rectum. A small number of circular whitish erosions, apparently associated with the solitary follicles.

Thorax. Of the left lung, the ventral and cephalic lobes are interspersed with small regions of collapse. The remainder of the lobes very emphysematous and hyperænic. Of the right lung, the anterior half (i. e., including cephalic, ventral, and adjacent portion of principal lobes) hepatized, covered by a thin false membrane, gluing the various lobes lightly to each other and to chest wall. The diseased lobes show the regular mottling in the upper, dorsal portion. As we proceed towards the ventral portion the mottling is less distinct, the tissue firmer and interspersed with small, irregular, necrotic foci. The smaller bronchi contain a thick, whitish pus. In ventral lobe a portion of the parenchyma as large as a marble completely converted into a grayishyellow hemogeneous mass. Of the principal lobe about one-third or one-fourth hepatized. The mottling of surface very regular. On section grayish, circumscribed areas one-half an inch in diameter interspersed. Over these masses the pleura is converted into a wrinkled, roughened, hide-like membrane.

Trachea and bronchi contain small quantities of foamy liquid intermingled with yellowish particles. Bronchial glands barely enlarged, firm ; some lobules pale, others reddened. 
Bacteriological notes. At the autopsy an agar tube was inoculated with a platinum loop lightly rubbed over the pleural exudate. On the following day a thin grayish growth with condensation water clouded. Examination of hanging drop and stained coverglass preparations shows only swine-plague gernis.

In coverglass preparations of hepatized lung tissue a large number of germs resembling swine-plague bacteria were seen.

With a bit of hepatized lung tissue, a rabbit was inoculated subcutaneously at 2 P. M. The rabbit was dead next morning at 8 A. M., i. e., in less than is hours. In spleen, liver, and blood preparations numerous polar-stained swine-plague germs present. An agar culture from heart's blood contained only swine-plague germs.

From the peritoneal exudate of pig, consisting of cells and fibrin and numerous bacteria of several varieties, two agar plate cultures were made. On Plate A one large colony of spore-bearing bacilli and one small colony of swine-plague germs. Plate B, completely overgrown by the spore-bearing bacillus.

A bouillon culture from the exudate contains streptococci and swine-plague bacteria. Agar plate cultures were made therefrom and both germs isolated.

At the same time a large rabbit was inoculated subcutaneously with a bit of the exudate Dead in Is hours. Stained coverglass preparations of blood from heart, spleen, and liver tissue show polar-stained swine-plague germs. An agar culture from the blood contains only swine-plague germs.

From the spleen, after thoroughly scorching through the exudate, two agar plate cultures and a bouillon culture were made with bits of spleen pulp. The bouillon culture contained only swine-plague germs.

The swine-plague bacteria were thus obtained from lungs, pleura, spleen, and peritoneal exudate, while hog-cholera bacilli, which were looked for with the greatest care, could not be found."

$\$ 50$. Differential diagnosis. In sporadic cases, swine plague is to be differentiated from broncho-pneumonia due to other causes than the swine-plague bacterium. Pneumonia of a non-specific nature is often associated with deaths due to dietary or other causes.

In epizoötics or outbreaks it is to be distinguished from hog cholera when there is accompanying catarrhal pneumonia. To make a positive diagnosis it is usually necessary to depend upon the results of the bacteriological examination.

Pneumonia resulting from lung worms, (Strongylus paradoxus) can be distinguished by a careful examination of the contents of the bronchioles. 
In case of coexistence of hog cholera and swine plague a bacteriological examination is necessary to determine the presence of the two diseases owing to the possibility of an accompanying or terminal pneumonia with hog cholera. The question has arisen as to whether the presence of $B$. septicaemiae hemorrhagicae in the hepatized lung constitutes a diagnosis of swine plague. As understood at the present time it would seem that the presence of this species of bacteria would indicate the nature of the disease. It must be remembered, however, that bacteria not readily distinguishable from the swineplague organism exists in the normal upper air passages from whence it could be brought into the diseased lung and in such a case it might appear as a secondary element only, or it might have been primarily the cause of the lesions. It is not improbable that epizoötics may start from these sporadic cases, although conclusive proof of this is still wanting. It seems, however, that the presence of this organism in the lung tissue of a sporadic case should be considered in the light of the distribution of these organisms and not necessarily as the starting of an epizoötic.

$\$ 5$. Prevention. Recent investigations show that outbreaks of swine plague are much more extensive than heretofore supposed. The present knowledge of this disease indicates, that the adoption of measures for its prevention is quite as important as for hog cholera. In general the measures to be adopted and followed, and the rules to be observed in the prevention of epizoötic swine plague are practically the same as those for the prevention of hog cholera. It will be seen from the comparison of the two species of bacteria that the bacillus of hog cholera is a more hardy organism than that of swine plague, Thus the swine-plague bacterium is destroyed more rapidly by drying and will live a much shorter time in the soil. However, the channels through which it may gain access to a herd are practically the same, and every precaution suggested in reference to hog cholera, is applicable to swine plague. It is believed that the time during which a field, hog yard or pen should be kept free from swine after the appearance of the disease can, with safety, be shorter after swine 
plague than after hog cholera. In any case several months should elapse before the yards or pens are reoccupied. If the disease appears, the well animals should be promptly separated from the sick and placed in suitable pens or yards-protected against subsequent infections, and given an abundance of wholesome food and water. It is well to remove the sick animals to other pens. The thorongh disinfection of the infected pens should be insisted upon before they are again occupied.

$55^{2}$. Specific treatment. For a number of years investigations have almost constantly been under way in the United States Bureau of Animal Industry, for the purpose of finding some method by which this disease could be successfully and specifically treated when introduced into a herd. Drugs and medicines have been tried, preventive inoculations and injections of toxins have been made. The serum therapy which has afforded relief in certain other diseases has been and is now being tested with somewhat farorable results, yet we do not see that a specific therapeutic agent has been demonstrated. In view of this fact, it becomes necessary to apply with renewed zeal our present knowledge of the nature of the malady and endeavor to prevent its occurrence or reappearance by keeping the animals under the best possible conditions. Prevention of the disease seems to be the best remedy.

$\$ 53$. Swine plague in rabbits. In 1894 , Smith and Moore described the appearance of the effect of swine-plague bacteria in rabbits and also the effect of resistance on the part of the rabbit on the form of the resulting lesions. As this disease is caused by a bacterium belonging to the septicæmia hemorrhagica group, it seems desirable that the results above referred to should be restated. The appended paragraphs are taken from the report of these investigations.

"Among the forms of disease observed after the subcutaneous inoculation of rabbits with swine-plague bacteria from different sources (epizoötics) are the following :

I. Septicæmia.

2. Peritonitis.

3. Pleuritis (usually with pericarditis). 
4. Pleuritis (usually with pericarditis) and peritonitis.

5. Local lesion only.

In the septicæmia, death ensues within eighteen to twentyfour hours. The local lesion produced at the seat of inoculation is slight. Bacteria are abundant in the parenchyma (blood vessels) of the various organs. In the form characterized by peritonitis death ensues in three to seven days. The local lesion, which in all these forms of disease increases in extent with the prolongation of the life of the animal, is here characterized by more or less suppurative infiltration of the skin and the subcutis. The peritonitis in its earlier stages is characterized by punctiform hemorrhages on the cæcum and a fibrinous or cellular exudate. It always contains immense numbers of bacteria. When pleuritis is also present the exudate usually involves the pericardium as well. It varies in amount according to the duration of the disease and is essentially the same as the peritoneal exudate.

The form characterized by pleuritis and pericarditis without peritonitis is interesting in so far as the seat of inoculation does not explain the localization, for, in every case, the inoculation was made in the region of the abdomen. The lungs may become hepatized secondarily through invasion from the pleura if the animal lives long enough.

Lastly, the form of disease in which the only localization is a very extensive suppurative infiltration associated with hemorrhage and oedema of the subcutaneous tissue is not common.

It should be stated that the cultures from the same outbreak continued to produce the same form of disease in rabbits until modified by age. The maintenance of a certain uniform virulence for years is well exemplified by a variety isolated in the summer of 1890 . This variety was fatal to rabbits within twenty hours when first isolated, and this degree of virulence has maintained itself up to the present, a period of nearly four years."

$\$ 54$. Modifications of the septicaemia type by increasing the resistance of rabbits. By the infection of sterilized cultures which increase the resistance of rabbits, Smith and 
Moore were able to produce nearly all the pathological variations which follow the inoculation of natural races of swine-plague bacteria as isolated from outbreaks. This modification of the septicaemia type is not fortuitous, for among the large number of rabbits inoculated during the past three and one-half years with the culture employed, none have survived twenty to twenty-four hours. Whenever the course of the inoculation disease in rabbits departed from this rapidly fatal type, it was due to some preliminary treatment of the rabbit.

"'The degree of resistance determined quite regularly though not invariably the form of the disease. This degree was measured by the relative quantity of the protective material (sterilized cultures, sterilized blood, and blood serum) injected. The grades of disease induced range themselves in the following order :

I. No resistance-acute septicaemia.

2. Slight resistance-peritonitis.

3. Increased resistance-pleuritis and pericarditis with or without secondary pneumonia.

4. Higher degree of resistance-pleuritis and peritonitis.

5. Still greater resistance-irregular lesions in the form of abscesses, subcutaneous and subperitoneal.

6. Nearly complete immunity. Very slight reaction at the point of inoculation.

Most of the cases cited below as illustrating these modified forms of the septicaemia type belong to the series of immunizing experiments of the preceding article. To this the reader is referred for additional illustrations.

First degree of resistance-peritonitis.-Rabbit No. I 2 received $7 \mathrm{cc}$. of bouillon culture of swine-plague bacteria sterilized by heat. Subsequently with a control rabbit it was inoculated with a minute dose of swine-plague bacteria under the skin. The control died within eighteen hours, the treated rabbit in three days. The macroscopic changes were linited to the point of inoculation and the peritoneum. At the former there was a purulent infiltration of the subcutis, $I .5 \mathrm{~cm}$. in diameter, with dilatation of surrounding blood vessels. The peritonitis was characterized by an exudate of a slightly viscid character covering liver, spleen, and caecum, and made up of fibrin, leucocytes, and immense numbers of bacteria. 
Second degree of resistance-pleuritis and pericarditis. - Rabbit No. 38 was treated before inoculation with $4.5 \mathrm{cc}$. of a sterilized suspension of agar cultures of swine-plague bacteria in 3 doses. Together with a control rabbit, it received under the skin the equivalent of $0.001 \mathrm{cc}$. of a fresh bouillon culture of swine-plague bacteria. The control died in twenty hours. The treated rabbit died six days after inoculation. At the point of inoculation there was a purulent infiltration of the subcutis 3 cm. in diameter. The abdomen and abdominal viscera were free from macroscopic changes. In the thorax, the pleural cavity was lined with a grayish, friable exudate consisting of round cells and bacteria. Lungs hyperaemic and only partly collapsed. Pericardium also covered with a slight exudate.

Third degree of resistance-pleuritis (pericarditis) and peritonitis. - Rabbit No. 15 received in the ear vein 12 cc. of a sterilized bouillon culture of swine-plague bacteria. It was inoculated subcutaneously with virulent swine-plague bacteria May 26 , and died June 3 , eight days later. The control rabbit died within eighteen hours. The following changes were observed:

A purulent infiltration into the subcutaneous tissue at the point of inoculation extending over an area $6 \mathrm{~cm}$. in diameter. The superficial layer of the subjacent nuscle discolored. Surrounding the area of infiltration the blood vessels were injected. The caecum and liver were covered with a very thin grayish exudate, which also appeared on and between the coils of the intestine. Spleen not enlarged.

The right lung and chest wall covered with a thin grayish exudate. In the cephalic lobe, two small areas of consolidation; principal lobe hyperaemic. The left pleural cavity lined with a quite thick membranous exudate, which covered the entire surface of the lung. On the dorsal surface of the principal lobe a mass of lung tissue $2 \mathrm{~cm}$. in diameter, firm and of a yellowish-gray color. The remaining portion of the principal lobe hyperaemic; cephalic lobe in a state of collapse.

Pericardium covered with a thin cellular exudate.

Higher degrees of resistance.-None of the treated animals which have come under our observation, have succumbed to a mere extension of the lesion produced at the point of inoculation as is occasionally observed after inoculation with certain varieties of swine-plague bacteria found in nature. There have been noticed, however, certain peculiar localizations resembling those produced in the subcutis after inoculation, and in 
a few cases the local lesion persisted a considerable length of time. It was quite severe in all fatal cases in which the disease was prolonged several weeks after inoculation, although the real cause of death was due in all such cases to localizations on one or more of the serous membranes. The peculiar forms of disease may be grouped as follows :

(a) Persistence of local lesions. - Rabbit No. 50 received in the abdominal cavity $3.5 \mathrm{cc}$. of the sterilized suspension of agar cultures in 3 doses. It was subsequently inoculated beneath the skin with $0.001 \mathrm{cc}$. of a bouillon culture of swine-plague bacteria which produced a large local swelling. On February 25, 1S92, nearly eight months after its inoculation it was chloroformed. The only lesion found was in the subcutaneous tissue. At the point of inoculation the skin was sloughed over an area of $3 \mathrm{~cm}$. in diameter. This denuded surface was covered with a thick scab. The subcutis beneath the scab and surrounding the ulcer was infiltrated with pus. A stained cover-glass preparation showed swine-plague bacteria. No other lesions were found.

(b) Sub-peritoneal abscess.-Rabbit No. 16 was injected intravenously with $16 \mathrm{cc}$ of sterilized bouillon cultures of swine-plague bacteria. After some days it was inoculated beneath the skin with o.oor cc. of a fresh bouillon culture of virulent swine-plague bacteria. The control rabbit died within twenty hours. Rabbit No, 16 showed no ill effect from the inoculation for several months when it was noticed that it was becoming emaciated. It died June II, IS92, one year and six days after its inoculation with an enormous subperitoneal tumor.

(c) Multiple abscesses under the skin.-Rabbit No. 439 received into the ear vein in three injections $4 \mathrm{cc}$. of sterilized blood from a swine plague rabbit. Later it was inoculated subcutaneously with $0.001 \mathrm{cc}$. of a bouillon culture of virulent swine-plague bacteria. The control rabbit died within twenty hours. Two months after the inoculation it was noticed that this rabbit with others was suffering from a large number of subcutaneous abscesses."

\section{REFERENCES.}

I. DE Schweinitz. Serum therapy. Proceedings Society for the Promotion of Agricultural Science. I896. p. 47.

2. DE SCHWEINITz. The serum treatment of swine plague and hog cholera. Bulletin No. 23. U.S. Bureau of Animal Industry. I899.

3. LOEFFLER. Arbeiten a.d. Kaiserlichen Gesundheitsamte. Bd. I ( $\mathrm{IS8}_{5}$ ) S. 51 .

4. MOoRE. Pathogenic and toxicogenic bacteria in the upper air passages of domesticated animals. Bulletin No. 3. U.S. Bureau of Animal Industry. IS93.

5. MOORE. Concerning the nature of infectious swine diseases in the State of New York with practical suggestions for their prevention and treatment. Report of the New York State Commissioner of Agriculture. 1897 . 
6. SмiтH. Preliminary investigations concerning infectious pneumonia in swine (Swine plague). Annual Report Bureau of Animal Industry U.S. Dept. of Agriculture. ISS6. p. 76.

7. Smith. Special report on swine plague. Bureau of Animal Industry. U.S. Dept. of Agricutture. ISgr.

S. SMITH AND MOORE Experiments on the production of immunity in rabbits and guinea pigs with reference to hog cholera and swine plague bacteria. Bultetin No.6. Bureau of Animal Industry, U.S. Dept. of Agriculture. 1894 . p. 65.

9. WELCH AND CLENiENTS. Remarks on hog cholera and swine plague. First International Congress of America. Chicago. 1893.

\section{HEAIORRHAGIC SEPTICAEMIA IN CATTLE.}

\$55. Characterization. Hemorrhagic septicæmia in cattle is determined by an acute attack usually running a rapid course and terminating fatally. The lesions consist largely of hemorrhagic areas more or less widely distributed throughout the body and due to the presence of a specific microorganism.

\$5. History. In 1879 , Bollinger described under the name of Wild und Rinderseuche an epizoötic disease which killed a large number of wild boars and deer in the Royal game preserves of Munich. After the disease in these animals had died out, the domestic cattle in the neighborhood began to die of the same or a very similar affection. He reports it to be sudden in its onset and rapidly fatal in its course, with a mortality of 90 per cent. Death occurred in from 12 hours to a few days after the first appearance of symptoms.

Two forms are described, (I) an exanthematous and (2) a pectoral. The post-mortems showed in the exanthematous form large and small hemorrhages disseminated throughout the muscles and viscera. The intestines always exhibited large numbers of ecchymotic areas, while the submucous tissue was infiltrated with a serous exudate. Large hemorrhagic tumors infiltrated with serum were abundant in the subcutaneous tissue and penetrated the muscles. The mucous membranes of the tongue, larynx and pharynx and the lymphatic glands of these regions were swollen and infiltrated with more or less bloody serum. In the pectoral form, a hemorrhagic lobular pneumonia, 
with considerable infiltration into the interlobular tissue of a serofibrinous exudate was present. The pleura was infiltrated and inflamed and covered with a fibrinous exudate. The pleural cavities contained from two to twenty-five litres of liquid. At the same time there existed a certain degree of hemorrhagic enteritis and the widely disseminated hemorrhagic lesions common to the preceding form.

In 1885 , Kitt studied an epizoötic disease in cattle and swine in Sincbach. From this disease he isolated a short, polar staining, non-motile, rod shaped organism, fatal to cattle, horses, pigs, sheep, goats, dogs and rabbits. In the fol lowing year Oreste and Armanni reported a destructive disease of young buffaloes in Italy with symptoms and lesions similar to those reported by Bollinger and Kitt. This disease had been known in Italy for a century or more where in certain districts it is reported to have recurred with great regularity destroying both old and young animals. In the same year (1886) Poels described a septic pleuro-pneumonia in calves which prevailed in the vicinity of Rotterdam. It was of a septicaemia nature. From the organs he obtained an organism belonging to the Bacillus septicaemiae hemorrhagicae group of bacteria. In I 889 , Jensen described a similar disease affecting calves in Jutland. In I889, Piot reported the presence of "barbone" in the buffaloes and domestic cattle in Egypt. In some districts 40 per cent. of the horned cattle are said to liave died in a single year. It is reported as being more prevalent in the wet season.

In 1890 , Van Ecke described a hemorrhagic septicaenia in cattle in Dutch India, particularly in Java, the lesions of which were similar to those first described by Bollinger. The specific organism was virulent for rabbits, mice, turtle doves, calves, horses and swine; sheep and asses were almost immune. In the following year Galtier described the same disease imported from Algiers to Lyons. A number of other investigators, among whom may be mentioned Reischig, Bongarts, Jakobi, Buch, Janson, Guillebeau and Hess, Von Ratz, Fischer, Lignières and others, have studied and reported cases of this disease. In 1890 , Nocard isolated from cases of broncho- 
pneumonia in American cattle landed at La Villette, France, an organism similar to that described as the cause of septicaemia hemorrhagica. In 1896 , Smith called attention to a similar organism which he found in cases of sporadic pneumonia in cattle ; he does not think, however, that this organism was primarily the cause of the disease, giving it a secondary place in the etiology. As early as 1891 , Smith published the discovery of the presence of an organism morphologically and in its cultural characters identical with that of swine plague in the upper air passages of healthy swine. The same year Moore reported the presence of a like organism in the upper air passages of cattle, horses, sheep, dogs, and cats. The following year, Fiocca described a pathogenic bacillus resembling that of rabbit septicaemia in the saliva of cats and dogs.

Hueppe proposed the name Bacillus septicaemiae hemorrhagicae for this group of organisms and septicaemia hemorrhagica for the disease they produce. While there may be objections to this unifying name, there seems to be no serious reason for not accepting it as a working hypothesis. In 1898 , Fennemore described under the name of "Wild and Cattle Disease" a malady occurring in Eastern Tennessee. Its serious nature caused an investigation to be made by the Tennessee Agricultural Experiment Station in which it was recognized by Nörgaard, who assisted in this investigation, as the same disease described by Bollinger in 1878 . Fennemore states that it has occurred to a considerable extent in his practice. In I9OI, it was carefully studied by Wilson and Brimhall for the Minnesota State Board of Health. They report 60 cases of this affection which they have examined in cattle in the state of Minnesota. It is from their report that we draw very largely for the symptoms and morbid anatomy of this disease in cattle.

$\$ 57$. Geographical distribution. It will be seen from the history that this disease is a wide spread malady occurring in nearly every country. It appears to be quite prevalent in the western part of the Mississippi valley.

$\$ 58$. Etiology. Septicaemia hemorrhagica in cattle is caused by an organism belonging to the group of bacteria designated by Hüppe as the hemorrhagic septicaemia group 
and specifically named as Bacillus bovisepticus by Kruse. This according to Migula's classification, should be Bacterium bovisepticum. A brief description of the organism as given by Wilson and Brimhall is appended.

"The organism has a strong tendency to show polar staining in tissues and to form chains of much shortened individuals in liquid media which causes it to be mistaken in examinations of a single specimen for a diplococcus or a streptococcus. Sometimes in cover-glass preparations from solid organs and very frequently in those from body fluids and liquid cultures, the bacteria were found in chains of three to twelve individuals. In cover-glass preparations the bacteria are from $0.6 \mu$ to $0.8 \mu$ broad and from 1.0 to $1.5 \mu$ in length. In tissues which have been fixed in 96 per cent. alcohol, they are somewhat smaller. In cultures, especially in fluid media, they are apt to be much smaller and approach diplococci in appearance. The ends are rounded. In stained preparations directly from the tissues most of the bacteria have the ends intensely stained and the central portion but faintly so. In sone chains in rapidly growing broth cultures this is not the case, many of the individual bacteria being evenly stained throughont and somewhat pointed at the ends. They do not retain the stain by Gram's method. The organism is nonmotile. It is aerobic, but prefers the depths rather than the surfaces of the media. It grows best at the body temperature and more slowly at room temperature. In plain and dextrose broth a heavy growth appears in 24 hours. In Dunham's solution a small amount of indol is formed in 48 hours. No coagulation of milk. On Löfflers blood serum, direct from the diseased tissues, it failed to grow well. On potato no appreciable growth has been obtained. In gelatin plates small, granular white to slightly yellowish colonies appear after 48 hours. In gelatin stab cultures a light growth occurs on the surface, while along the needle tract numerous colonies like those in the deep portions of the plate cultures develop. The bacteria are destroyed in fluids at $58^{\circ} \mathrm{C}$. in 7 or 8 minutes, by 1 to 5,000 mercuric choloride in 1 minute, and by a solution of lime water as weak as 0.04 per cent. almost immediately."

\$59. Symptoms There is little opportunity to determine the symptoms. The animals observed at the onset of the disease by Wilson and Brimhall, appeared dumpish and out of sorts. There is sudden stopping of the milk secretion in milch cows. As a rule the affected animals refused food. Of the few that make an attempt to eat, those with affected throats were unable to swallow except with much difficulty. These cases also breathed very heavily. The animals showed marked disinclination to move and when incited to do so, exhibited stiffness, 
and in some instances actual lameness. Some of the animals dropped to the ground and died in a short time, apparently without pain. Others were down when first observed to be sick and lived for several hours in great pain as indicated by groans, and spasms of the muscles. The paroxysms of pain were apparently intermittent. There was extremely rapid loss of flesh in the animals that were sick more than one day.

Opportunities for the accurate determination of temperature at the beginning of the disease were very few. It appears that an initial temperature of $105.6^{\circ} \mathrm{F}$. was present followed by a rapid decline. Painful oedematous swellings about the legs, shoulders and under the throat were noted as early symptoms. The intestinal discharges were often streaked with blood. In other cases the feces were black, tarry or of a bloody, serous nature.. Bloody urine and a bloody serous discharge from the nose were present in some of the cases. The vaginal and rectal mucous membranes were intensely congested.

The marked swellings of the face, stomatitis, glossitis, and convulsive movements of the jaws in the pneumonic form of the disease described by European writers, more especially Bollinger, were not observed by Wilson and Brimhall in any of the recent cases.

$\$ 60$. Morbid anatomy. The characteristic lesions of the disease are widely distributed areas of hemorrhage, varying in size from a pin point to several centimeters in diameter. They vary in color from light red to almost black. They are frequently accompanied with a sero-fibrinous exudate, usually yellow, but occasionally dark red in color. The hemorrhagic areas in the animals just dead are not so dark as those in animals that have been dead for some hours. The large areas, some centimeters in diameter, are apparently due, in some instances, to single hemorrhages infiltrating an extensive mass of tissue, and in others to a number of minute hemorrhages closely placed and partially coalescing. Gas is not present in the subcutaneous connective tissue except cases where extensive post-mortem changes have occurred.

There is excessive fullness of the vessels of the subcutaneous connective tissue in the acute cases, especially in 
those animals which are not killed by bleeding. In animals which live until emaciation is marked, there is no engorgement of the vessels.

All cases show some hemorrhagic areas in the subcutaneous tissue, though the number and size of these vary greatly in the different cases. Some animals show not over a dozen areas between two and three centinteters in diameter, though many minute ones are present. In other animals, on removing the skin, hemorrhagic areas are found in great numbers and so extensive that a large fraction, possibly one-eighth, of the body surface appears to be involved. The large hemorrhages in the subcutaneous connective tissue appear to be of the composite type noted above.

The location of the superficial lesions varies in different animals. In most cases the parts about the shoulder are most affected, -in some the throat, and in others the digital region. A few animals show marked lesions in the gluteal and inguinal regions.

At first sight the muscle tissue in some cases seems to be much involved. A closer examination, however, usually shows that while some of the minute hemorrhages are in the muscle proper, the larger ones are in the intermuscular connective tissue. They are usually accompanied by a considerable quantity of yellowish or blood stained serous exudate. The intermuscular connective tissue appears to be quite as much involved as the subcutaneous connective tissue.

The lymphatic glands are frequently, though not uniformly nor constantly enlarged. Those that are enlarged are oedematous and often hemorrhagic. The most constantly and seriously affected are the cervical and prescapular.

The nasal mucous membrane in some cases is congested, and a bloody serous discharge from the nostrils is present in a few cases. The tissues around the larynx are hemorrhagic and infiltrated with blood stained serum. The mucous membranes of the larynx and trachea are more or less congested and covered with a frothy mucus, sometimes streaked with blood. In some instances no lesions are observable in the larynx and trachea. The lungs are in general almost free 
from evidence of disease. A few showed a small number of hemorrhagic areas, pyramidal in shape with their bases on the pleura. In most cases the parietal pleura is studded with small hemorrhages. The diaphragm sometimes contains very large hemorrhagic areas.

The pericardial sac usually shows small, sometimes very numerous hemorrhages in the walls, and in many instances contains a blood stained serum.

The heart walls, with but few exceptions, contain large and small hemorrhages. These sometimes extend deep into the muscle. Similar areas of hemorrhage are also visible in the endocardium. The heart contains blood clots, post-mortem in formation.

The blood, in animals just dead, is somewhat lighter than normal in color. When post-mortem changes set in, the blood is darker in color, but reddens on exposure to the air.

The spleen shows on its surface a few small hemorrhagic areas. It is usually normal in size, color and consistency, except where post-mortem changes have taken place.

Stomatitis and glossitis are rarely observed. The pharynx is usually congested.

The stomach walls contain few or many hemorrhagic areas. These are sometimes extremely large, especially on the third stomach. As a rule the larger hemorrhages penetrate the entire thickness of the walls. The smaller ones are confined to the subserous or mucous coats. The stomach contents are apparently normal.

The intestinal walls are constantly affected. Hemorrhagic areas involving all the coats are frequently present. Smaller ones, visible only from the inner or outer surface, are always present. General enteritis and peritonitis are occasionally observed. Localized enteritis is frequent.

The bowel contents are in some cases dark and tarry ; in others the feces are apparently normal in color and consistency but streaked with bloody mucus.

The kidneys are usually but slightly affected. When lesions are present they are but pin point in size and mostly confined to the cortical substance, though a few are found in 
the walls of the pelvis and ureters. The urine is bloody in some instances.

The vaginal mucus membrane is congested in many cases. Wilson and Brimhall saw one animal that was four months pregnant, which exhibited small areas of hemorrhage in the placental membranes.

The udder is congested in some cases, in one instance it appeared as one mass of blood.

The central nervous system was examined in but three cases. The first two showed hemorrhages in the dura. An examination of a small portion of the cord showed no lesions. Two cases showed hemorrhages in all of the joint surfaces.

Portions of the subcutaneous tissue, skeletal muscles, ly mphatic glands, lung, heart wall, stomach wall, and spleen were fixed in 95 per cent. alcohol and in 4 per cent. formaldehyde solution and stained by various methods by Wilson and Brimhall. In general, the lesions found were enormous extravasations of blood, some recent and some showing coagulation of fibrin. In the areas of less recent hemorrhage, the surrounding tissues showed varying degrees of ordinary coagulation necrosis. This was particularly marked in the affected muscles, lymph glands, and portions of the lungs. In the borders of such necrosed areas leucocytic infiltration was not infrequent. In the spleen in which the hemorrhagic areas were neither numerous nor large; there was in some instances, an apparent destruction or shrinkage of the parenchyma.

A very important feature in this disease has been brought out, namely, that is it necessary, in order to obtain cultures of the bacterium producing it, that the media should be inoculated at once. It is evident from the literature that with this precaution cultures should invariably be obtained. The writer had occasion to investigate an outbreak of disease of considerable proportions in Central New York where the lesions corresponded exactly with those more recently described by Wil son and Brimhall. Allthough a very large number of coverglass preparations from the blood and different tissues were made and stained by various solutions and methods, and many tubes of bouillon, agar and gelatin were inoculated, bacteria were not found in the tissues of any of the animals examined. 
$\$ 6$ r. Differential diagnosis. Septicaemia hemorrhagica in cattle must be differentiated from anthrax, symptomatic anthrax, and the affection known as "corn stalk disease." Death from this disease must also be distinguished from those due to certain accidental causes, poisoning, or the effect of over eating of grain or green fodder (hoven). The suddenness with which the animals may die from all of these causes renders the symptoms, should they be observed, of very little value in making a diagnosis. It is necessary, therefore, that in all cases, especially with the first animals to die that careful post-mortem and bacteriological examinations should be made.

In case of septicaemia hemorrhagica, the cultures will usually reveal the presence of Bact. bovisepticum. The lesions hemorrhagic in nature.

In case of the "cornstalk disease" the lesions may consist of small hemorrhages (petechix) especially of the serous nembranes and heart. The cultures will be negative.

With authrax and symptomatic authrax, the specific bacteria of these diseases will be found.

$\$ 62$. Prevention. When this disease occurs it is important to remove the unaffected animals to other fields or enclosures. It is well to divide them into small groups if possible. The carcasses of animals that die should be burned or buried deeply with a good covering of a disinfectant such, for example, as quick lime. Should death occur in a stable, all contaminated litter should be burned and the floors, mangers and walls thoroughly disinfected.

Experiments have been made directed towards protective inoculation, but thus far the results have not been sufficiently satisfactory to warrant the recommendation of the methods.

\section{REFERENCES.} I 878 .

I. BOLIINGER. Ueber eine neue Wild-und Rinderseuche. Munchen.

2. FenNmiore. Wild and cattle disease. Jour. of comp. med. and vet. archiv. Vol. XIX (1898) p. 625.

3. GALTIER. Noveaux faits tendant a etablir que la pneunoenterite infectieuse existe sur les grands et les petits ruminants en algerie. Recueil de med. veter., 7 serie., Vol. VIII. I891. p. 97. 
4. HuEPPE. Ueber die Wildseuche. Berlin. klinische Wochenschrift. 1886. p. 753 .

5. KiтT. Ueber eine Experimentelle, der Rinderseuche (Bollinger) ähnliche Infektionskrankheit. Sitzungsberichte der Gesellschaft für Morphologie und Physiologie in München, I. I885. p. 140.

6. PIot. Le Barbone du Buffe. Bulletin d. l'Instit Egyptian. I889. 7. POELs. Septische Pleuropneumonie der Kälber. Fortschr. d. Med. 1886. p. 358.

S. Wilson AND BRIMHALL. Sixty cases of haemorrhagic septicaemia in cattle due to Bacillus bovisepticus. Report State Board of Heallh of Minnesota. rgor. (Very full bibliography.)

FOWL CHOLERA.

\$63. Characterization. This is an infectious disease of fowls caused by bacteria, and transmissable by cohabitation and inoculation. It is determined by a high fever, great weakness and prostration, and usually terminates in the death of the affected bird. It is reported that it attacks all varieties of domesticated poultry (chickens, ducks, geese, pigeons, turkeys), and caged birds such as parrots and canaries. It also attacks some species of wild birds. It is communicable by inoculation to rabbits and mice. Guinea pigs are not very susceptible.

$\$ 64$. History. This disease is mentioned in some of the oldest works treating of the disease of animals. Fowl cholera was studied by Chabert in 1782 who regarded it as a form of anthrax. Since 1825 , it has been frequently observed in France where it caused enormous losses in I 830 , in 1850 and in 1860 . About 1830 , it became known in Russia, Bohemia and Austria. In $185 \mathrm{I}$, Benjamin considered it to be a contagious disease but remarked that people and dogs might consume with impunity the meat of affected fowls. Delafond observed that it might be transmitted to birds and rabbits by using blood, secretions, and portions of the flesh. It was also recognized that the excrement plays an important part in the dissemination of the virus.

During recent years it has been observed in nearly all of the countries of Europe as well as in the United States. It has been reported from many places in the United States, but 
its presence seems to have been determined by scientific investigation in but a very few of these. Salmon investigated it in South Carolina in I879-80, and Higgins in r 898 reported it from Canada. Salmon gave special attention to vaccination and the effect of disinfectants in destroying the virus.

Perroncito was among the first to describe the specific cause of the disease. This was followed by the contributions of Pasteur, who, in ISSo, cultivated the bacterium in chicken broth and showed that its virulence might be reduced to such an extent that it could safely be used for vaccination. This is the first case in which a virulent organism was successfully modified in a laboratory and made to act as a vaccine. It was the forerunner to the preparation of vaccines for a number of diseases, more particularly for anthrax, black quarter, and rabies.

\$ 65. Geographical distribution. Fowl cholera seems to be widely distributed in Europe and it has been found in a few localities in the United States and in Canada.

$\$ 66$. Etiology. Fowl cholera is caused by a specific bacterium which is not distinguishable morphologically or in its cultural manifestations from the other members of the group of bacteria of which Bact. septicaemiae hemorrhagicae is the type. In this group are the bacteria of rabbit septicaemiae, swine plague, and Wildseuche. It is a small slightly elongated organism with rounded ends. In stained preparations from the tissues it exhibits a pronounced polar stain.

s 67. Symptoms. The symptoms described for this disease in Europe differ somewhat from those reported by Salmon. The period of incubation is placed by European writers at from is to 48 hours. In the case of 40 fowls inoculated by Salmon, it varied from 4 to 20 days the average period being 8 days. The duration of the disease also varies. Usually the sick birds stop eating or the appetite is lessened, though occasionally they continue to eat almost to the time of death. The earliest indication of the disease, is a yellow coloration of the urates. In health, these are a pure white though they are frequently tinted with yellow as the result of disorders other than cholera. 
Occasionally the first symptom is a diarrhea in which the excrement is passed in large quantities and consists almost entirely of white urates mixed with colorless mucus.

Very soon after the first symptoms appear the bird separates itself from the flock, it no longer stands erect, the feathers are roughened, the wings droop, the head is drawn down towards the body and the general outline of the bird becomes spherical or ball shaped. At this period there is great weakness, the affected bird becomes drowsy and may sink into a sleep which lasts during the last day or two of its life, and from which it is almost impossible to arouse it.

The crop is nearly always distended with food and apparently paralyzed. There is in most cases intense thirst. If the birds are aroused and caused to walk, there is at first an abundant discharge of excrement followed at short intervals by scanty evacuations.

With the beginning of diarrhoea the body temperature rises to 109 or $110^{\circ} \mathrm{F}$. The comb loses its bright hue and becomes pale and bloodless. In Europe the comb is described as dark blue, purple, or black, and some writers in the United States have referred to it in the same terms. Salmon reports that he has never observed a dark comb in the cases he has seen.

Diseased birds rapidly lose in weight, they are so weak that a slight touch causes them to fall over, and they walk with great difficulty. The fowls become very much emaciated. Death may occur without a struggle or there may be convulsive movements and cries.

This disease may rapidly run through a flock destroying the greater part of the birds i11 a week, or it may assume a more chronic form, extend slowly, and remain upon the premises for several weeks or months.

$\$ 68$. Morbid anatomy. The comb is pale and bloodless. The superficial blood vessels usually contain but little blood, and there are in most cases soiled feathers about the anus, to which the excrement may adhere in considerable quantity.

The liver is usually enormously enlarged, softened, with 
blood vessels engorged. The gall bladder is distended with thick dark bile.

The crop is usually distended with food, though no special lesions have been noticed here. The stomach viewed externally, often presents a number of circular discolorations about three millimeters in diameter, which on section are found to be extravasated blood. The small intestines are congested.

The rectum and cloaca usually present deep red lines upon their mucous membrane, evidently the first stage of inflammation, which results, in chronic cases, in thickening of the walls, especially of the rectum, the desquamation of the mucous membrane, and the formation of large ulcerated surfaces.

The mesentery is generally congested, often greatly thickened and reddened, and rendered opaque by inflammation. The ureters are distended with yellow urates; the kidneys seem engorged, and on section accumulations of the tenacious, yellow urates are frequently seen. The spleen is generally normal in size and appearance, though frequently enlarged and softened.

The pericardium is sometimes distended with effusions, in which case there is noticeable hyperaemia of the surface of the heart. The lungs are often, though not generally, engorged with dark blood; they are seldom, if ever, hepatized.

The blood vessels are sometimes filled with a firm clot, and contain but little liquid; at other times the blood does not coagulate at all. It seems to be those cases in which the duration of the disease is longest, that the blood loses its power to coagulate.

59. Differential diagnosis. Fowl cholera is to be differentiated (I) from a number of dietary disorders which cause the death of a large number of fowls. These cases are often thought to be chicken cholera and so reported by the owners. A diagnosis is to be made in the findings of a bacteriological examination. (2) Fowl cholera is to be differentiated from infectious leukaemia. There are a number of resemblances in the clinical history of the two diseases but 
there are marked differences in both the morbid anatomy and etiology. For a comparison of the lesions and specific bacteria of these two diseases see infectious leukaemia.

\$ 70. Prevention. Pasteur introduced a preventive inoculation or vaccine for this disease. Kitt has found that the eggs of fowls unknown to this disease possessed a substance somewhat similar to antitoxin. He immunized fowls by injecting them subcutaneously with from four to eight cubic centimeters of the white of such eggs. While exceedingly interesting this method does not seem practicable. Good sanitary conditions, isolation of the well from the sick fowls and thorough disinfection seems to be the most satisfactory procedure. It is important not to introduce the disease with newly purchased fowls or to expose healthy ones to the disease either at or in transportation to various poultry exhibits.

\section{REFERENCES.}

1. Higgins. Notes on an epidenic of fowl cholera and upon the comparative production of acid by allied bacteria. Jour. of Experimental Medicine. Vol. III. ( IS9S) p. 65I.

2. PeRroncito. Arch. furwiss, prackt. Thierheilkunde. IS79. p. 9.

3 PaSteur. De l'attenuation du virus du Cholera der poules. Comptes Rendus des Seances de l'Academie des Sciences. I8so. Vol. XCI. p. 673 .

4. PASTEur. Sur les maladies virulentes, et en particulier sur la maladie appelée vulgairement choléra des poules. Ibid. ISSo. Vol. XC. p. 239.

5. Salion. Annual Reports of the U.S. Commissioner of Agri. culture. $\quad$ ISSO. $-S_{2}$.

6. SALMON. The diseases of poultry. Washington, D. C. IS99. p. 232.

\section{ANTHRAX.}

Synonyms. Splenic fever; splenic apoplexy ; wool sorters' disease ; malignant pustule ; anthracaemia ; charbon ; mal-de-rote; Milzbrand; mycosis intestinalis.

S 7r. Characterization. Anthrax is an infectious disease occurring sporadically and is epizoötics in herbivora and omnivora and communicable to nearly all warm-blooded animals, and to man. It is characterized by the presence in 
the diseased tissues or liquids of Bacterium anthracis, by an enlarged spleen, blood extravasations and by local gangrene. It usually occurs in the acute form.

$\$ 72$. History. Anthrax is among the oldest of the known infectious diseases of animals. Descriptions of epidemics and epizoötics of this disease are given by Homer, Plutarch, Livy and other writers before the Christian Era. The Arab physicians designated it as "Persian Fire." Extensive outbreaks are mentioned in the literature of the fifteenth, sixteenth, seventeenth, eighteenth and nineteenth centuries. Chabert pointed out in 1780 that the various kinds or forms of disease, which had previously been described as independent affections, were all one disease. As late as I805, Kausch gave a good description of anthrax but denied its contagiousness. Delafond and Gerlach investigated very thoroughly ovine anthrax in $18+5$ and its contagiousness was experimentally shown by Gerlach. In I850, Heusinger published a very comprehensive treatise on anthrax, which dealt at length with its history and geographical distribution.

Much new information concerning the nature of anthrax was acquired during the fifth decade of the last century. In I855, Pollander announced the discovery, which he first made in I 849 , of minute unbranched rod shaped bodies in the blood of cattle dead of anthrax. Davaine observed similar bodies in I 850 . Then followed a long series of observations and somewhat controversial discussions on the bacterial origin of the disease, culminating in $\mathrm{s} 875$ by Robert Koch's careful description of the morphology of its specific organism including the spore formation. Colin, however, seems to have been the first to have called the organism a Bacillus and to have suspected the existence of spores. Toussaint, in 1880 , and Pasteur in $188 \mathrm{I}$, published results of investigations directed toward protective inoculation. Since that time, the literature on the cause, morbid anatomy and prevention of anthrax has become very extensive.

\$73. Geographical distribution. Anthrax is a widely disseminated disease. The continent of Europe has perhaps suffered most from its ravages. It occurs, also, in Northern, 
Eastern and Central Africa, where in recent years it has become a great plague. In Siberia it has caused fearful destruction, and in that country it is still known as the "Siberian Plague." It has frequently appeared in England. Russia, India and Australia are also infected. In the United States, it has been reported from at least fifteen states and territories. In fact there are very few, if any, countries where this disease has not been found. In the eastern part of this country it exists to a greater or less extent in certain localities. A knowledge of its specific cause with the methods of properly disposing of dead animals, isolation and disinfection as well as the preventive inoculations now in vogue, have made it possible to prevent widespread epizoötics. In America it is looked upon as a comparatively rare disease, excepting in certain infected districts.

S74. Etiology. Anthrax is due to the effect of the presence of a specific micro-organism known as Bacterium (Bacillus) anthracis. This organism is found in the diseased tissues and organs of affected animals. On account of its spores, it is very resistant to the normal destructive agencies in nature. Consequently when it is once introduced into a locality it

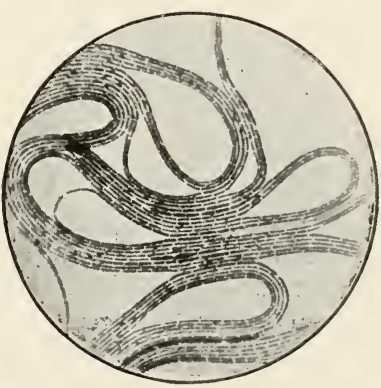

Fig. 10. Anthrax bacteria from an tends to remain there for impression preparation from a colony. many years, possibly causing from time to time a few cases of anthrax or serious epizoötics or epidemics. The spores are also frequently carried in the wool, hair, hide, hoofs and horns taken from animals sick or dead of anthrax. Thus the affection has been introduced into far distant localities.

Bacterium anthracis is a rod-shaped organism varying in length from I to $4 \mu$, but having a quite uniform breadth of about one micron. In a suitable medium it grows out in long 
flexible filaments, which combine to form thread-like bundles. When examined, the ends of the rods seem to be square cut. In preparations from animal tissues there appear sometimes to be slight concavities in the ends of the segments when two of them are united. In old cultures spores are formed. These are oval, highly refractive bodies held within the cellular envelope of the filaments, but later they are set free by the dissolution of this membrane. It stains readily with the aniline dyes and also by Gram's method.

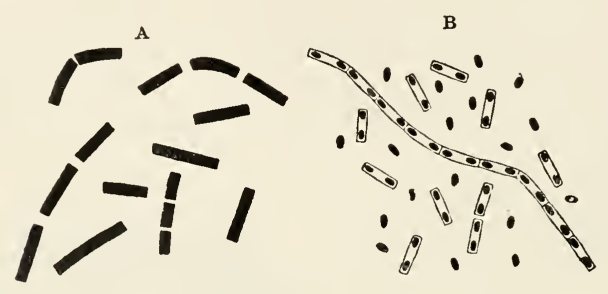

FIG. II. Bacterium anthracis. A. without spores. B. with spores.

The bacterium of anthrax itself is not an especially hardy organism. On the contrary it is easily destroyed by weak disinfectants and it has a low thermal death point. Its spores, however, are among the most hardy of bacterial life to resist chemical and thermal agents. They resist drying for months or years and often boiling for a half hour or longer does not destroy them. On this account it is very difficult to eliminate the virus from infected pasture lands, especially if they are wet or marshy.

As the spores may remain in the soil in a dormant condition for many years it sometimes happens that the disease does not appear until long after the introduction of the virus. Anthrax has been known to break ont among cattle grazing on a field in which the carcasses or hides from affected animals were buried many years before. Through some means the spores were able to get to the surface and contaninate the grass. The virus may be introduced with blood or 


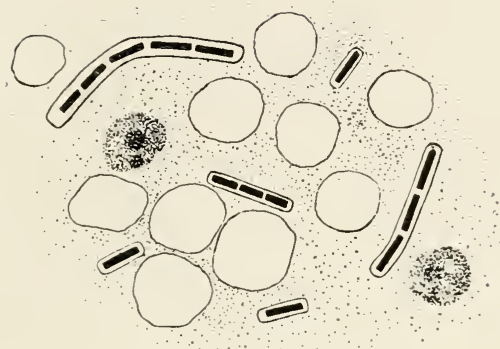

FIG. I2. Anthrax bacteria in a cover-glass preparation of blood showing chains and capsules.

bone fertilizers, hides, hair or wool from infected countries. When the extent of this traffic is realized, it is easy to understand how anthrax has been brought to this country and why it occasionally appears hese and there over a large part of the continent. Many outbreaks, as well as isolated cases, illustrating this common method of disseminationi are on record.

$\$ 75$. Animals attacked. Nearly all animals suffer from anthrax. The herbivora and rodents are most susceptible. It is interesting to note that the Algerian race of sheep are immune. A satisfactory explanation for this striking exception has not been recorded. It has been stated that a single bacterium introduced into the subcutaneous connective tissue of a guinea-pig or mouse is sufficient to kill. Cats, tame and wild rabbits, and hares are the next most susceptible species. It is stated that dogs, pigs, and foxes are very slightly susceptible. Rats, fowls and pigeons are reported to be immune. Fish and amphibia are rarely attacked.

$\$ 76$. Channels of infection. Three common modes of infection are recognized for anthrax, namely: through the digestive tract, by the skin and by the lungs. In cattle the infection seems to be largely through the alimentary canal; in horses and sheep by the skin or digestive tract; in men 
through wounds of the skin and the respiratory tract. There are, however, many exceptions to these usual methods.

(I.) Infection through the alimentary canal. This is the more common mode of infection in cattle. The resulting disease has been designated by various names among which are "intestinal anthrax," "fodder anthrax," "spontaneous anthrax," “'internal anthrax," “anthrax fever,' and anthrax without external manifestations. In these cases the infecting organisms, either the spores or the bacteria themselves, are taken into the body with food or drinking water. It is stated that the infection takes place in most cases in the sullall intestine, the mucosa of which, it is stated, need not necessarily be injured. It is highly probably that the gastric juice destroys most of the bacteria while the free spores are not injuriously affected by it. In infected districts the spores exist at or upon the surface of the soil and possibly on the blades of grass from which they are easily taken up by grazing animals. In cases thus infected the specific organisw has been introduced at some previous time either (a) by the burying of diseased animals in these fields, (b) by the use of infected tannery or slaughter house refuse as fertilizers, (c) by flooding from infected streams, or (d) by the bringing of the organism in the droppings of birds or other small animals which have fed upon anthrax carcasses. It is reported that the spores will find their way to the surface even when the dead animals have been buried at a considerable depth. There has been some controversy in the writings of Pasteur, Koch and Bollinger concerning the method by which the spores reach the surface. Pasteur supposed that they were brought by earth worms from the buried carcasses. Koch believed this impossible because of the low temperature of the ground at the depth at which the animals are buried. Bollinger has shown experimentally the possibility of Pastenr's views. Karliniski and others have found that the spores of anthrax may be disseminated by slugs, insects and larvae which are found on untanned infected skins.

(2.) Infection through the skin. In animals, this mode of infection occurs less frequently than in man. Anthrax 
produced in this way is usually characterized by local manifestations known as "carbuncle disease," or "malignant pustule." In this mode of infection the bacteria penetrate, through wounds in the skin and exposed mucous membranes, into the living tissues by means of infected utensils, the use of infected instruments, and the bites of insects, especially the house fly (Musca domestica.) Dalrymple has called attention to the spread of this disease among animals in the lower Mississippi Valley by means of the horse fly (Tabanidae.) In man many cases of the disease occur from the injuries or cuts made at the post-mortem of anthrax animals or by the infection of skin wounds while handling infected hides or wool. Malignant pustule is reported to be quite common among the employes of certain tanneries and upholstering establishments where hides and hair imported from infected districts or countries are used.

(3.) Infection through the respiratory tract. Faser, Buchner, Lemke, and other writers have shown experimentally that the disease can be produced by the inhalation of spores. In man this form of infection is quite common among the wool sorters. In Great Britain where much foreign wool is handled it has been reported as causing as many as 500 deaths annually.

$\$ 77$. Symptoms. In anthrax, the symptoms vary not only in different species of animals but also in different individuals according to the location of the disease. Again, there is often considerable variation when the lesions are apparently the same. The most characteristic features of the disease are (1) the suddenness of the attack, (2) the grave general disturbances, (3) high elevation of temperature, (4) a tendency to ecchymoses of the mucous membranes and (5) local manifestations, such as carbuncles and oedema of the skin, digestive disturbances, brain complications or difficult respiration. It sometimes happens that symptoms of metastatic infection are detected.

Anthrax has been classified according to its course as peracute, acute and subacute. It has also been divided accord- 
ing to the site of its manifestations as anthrax with visible localization, and anthrax without visible localization.

Anthrax without visible localization. This form is generally due to ordinary infection presumably by spores. It includes the peracute, acute, and subacute.

(1) The peracute or apoplectic anthrax gives rise to symptoms of cerebral apoplexy. The animal becomes suddenly ill, staggers about for a brief period and falls. There is often a bloody discharge from the mouth, nostrils and anus. Death usually ensues in from a few minutes to an hour. Usually there are convulsions. Sheep and cattle suffer most frequently from this form of the disease. They are often found dead. This is especially true in the beginning of an epizoötic.

(2) In the acute form the disease runs a somewhat slower course, lasting usually not to exceed twenty-four hours. The temperature rises rapidly to from 105 to $108^{\circ} \mathrm{F}$. With this there are signs either of congestion of the brain or of the lungs. If the brain is affected the animal becomes restless, excited, stamps the ground, rears in the air, bellows, runs to and fro and finally goes into convulsions followed by stupor and death. If the lungs are congested there is difficulty in breathing, more or less wheezing, panting, groaning, palpitation of the heart, small and frequent pulse, cyanosis of the mucosa of the head, bloody discharges, haematuria, staggering and finally convulsions and death from suffocation. Occasionally there is a partial remission of the symptom, followed by relapse. It has been observed that occasionally there are premonitory symptoms preceding the acute attack consisting of slight digestive disturbances and diminished vivacity.

(3) The subacute form is known as anthrax fever or intermittent anthrax. The symptoms are the same as in the other forms, excepting they are more sharply defined and the course is longer. The disease lasts from one to seven or eight days, the average being about forty-eight hours. The high temperature, the congestion of the lungs or brain complicated with intestinal disturbances, especially colic, are usually well 
marked. In epizoötics where the peracute or acute form ushers in the disease, the later cases usually are of the subacute variety. It often happens that the last animals attacked exhibit the subacute or more chronic form.

Anthrax with visible localization. These forms usually result from infection of the skin and mucous membranes. The lesions are spoken of as carbuncles and often there is marked local oedema of the skin. This form is common in man, horses and sometimes in cattle. It is reported to occur in other species. The carbuncles are circumscribed, cutaneous swellings which are at first hard, hot and painful. Later they become cold and painless with a tendency to become gangrenous. The oedematous tissue becomes doughy, cold and painless; frequently fluctuating swellings of the skin occur. The duration of this form of the disease varies from four to fifteen days. Ordinarily it is not so fatal as internal anthrax.

When the infection is on the nucous membrane the animal suffers from fever, dyspnoea, difficulty in swallowing, and cyanosis, together with the immediate local effects. Death occurs much sooner than when the disease is located on the skin. It is stated that dogs and swine suffer from this form more than from the more acute types.

In horses, anthrax usually runs an acute or subacute form. The first symptom is rise of temperature with a rapid, feeble pulse. Occasionally there are chills and muscular spasms. The nucosa of the head becomes cyanotic and lacrymation is often present. The animal has a dull, stupid look, appears to be stunned and walks with a staggering gait. In some cases there are symptoms of cerebral congestion such as restlessness or convulsions. Colic is a very characteristic symptom in the horse, otherwise the symptoms are the same as in cattle. Infection of the skin usually occurs on the hypogastrium, lower part of the breast, inner surface of the fore and hind quarters. Swelling of the hind quarters often causes lameness. Carbuncles of the mucous membrane of the tongue is said to occur rarely in these species.

In sheep and goats the disease is usually of the acute or apoplectic form. The animals appear as if suddenly stricken 
with apoplexy. If death does not occur within a very short time, symptoms heretofore described for this form of the disease may be recognized. Subacute anthrax is said to be very rare in sheep.

In swine, anthrax is ordinarily characterized by local lesions on the mucous membrane of the larynx or pharynx. The animals have a rise of temperature and the intermaxillary space is generally swollen. The swelling may spread along the trachea giving rise to difficulty in swallowing, hoarseness, cyanosis of the mucosa of the mouth, dyspnoea and rapid breathing. The animal shows signs of paralysis. Death occurs from suffocation. Frequently the tongue becomes the seat of the disease. Carbuncles occuring on the skin, especially of the back, have been described in this species.

In dogs and cats, the disease usually runs a very rapid course. The fact that they are usually infected by eating the meat of animals dead of anthrax causes them to suffer largely from the intestinal form. It has been stated that probably much of the so called antlirax in dogs was simply cases of ptomaine poisoning.

It is reported that in birds anthrax usually runs a very rapid and usually fatal course. Toward the end they stagger, tremble, or go into convulsions, and die with bloody discharges from the mouth, nostrils, and anus. From the first the birds are depressed and weak, their feathers ruffled and wings drooping. There is evidence of dyspnoea and bloody diarrhoea. Occasionally carbuncles appear on the comb, wattles, conjunctiva, tongue and extremities.

It has been stated that the milk from cows suffering with anthrax contains Bact. anthracis. The writer found in the examinations made in one epizoötic that the anthrax bacteria were present in considerable numbers in the milk just before or immediately after death, but they were not found in the milk of animals in the earlier stages of the disease.

$\$ 78$. Morbid anatomy. The nature and extent of the tissue changes depend upon the course of the disease. When experimentally produced it is ordinarily a septicaemia. This form often occurs in the domesticated animals that contract the 
disease naturally. The more common anatomical changes, except in the most acute cases and in the strictly localized lesions or carbuncles are: (1) Hemorrhages varying in amount from petechiae to blood extravasations, with more or less serous, gelatinous and hemorrhagic infiltration of the submucous, subserous and subcutaneous tissue ; (2) Enlargement of the spleen with parenchymatons inflammation of the liver and kidneys; (3) A dark tar-like condition of the blood; and (4) The presence in the tissues and blood of Bacterium anthracis. In the very acute cases these changes are often slight, while in the more chronic forms they are strongly marked. It is important to note that occasionally the usual changes indicated by the symptoms and the duration of the disease are not found on post-mortem examination. In one epizoötic, the writer has seen an animal dead from subacute anthrax in which the blood and tissnes were teeming with anthrax bacteria, yet the organs appeared to be perfectly normal. Other animals in the same ontbreak exhibited the more usual anatomical changes.

The blood is usually very dark and tar-like in appearance. The cutaneous capillaries are distended and frequently there are hemorrhages beneath the epidermis. The subcutis is sprinkled with ecchymoses. Frequently there are gelatinous effusions of a rather firm consistance and of varying size. The color also differs, ranging between a deep yellow and a yellowish brown. Often these oedematous areas are sprinkled with hemorrhagic foci. A simple serous oedema may occur.

The lymphatic glands may be hemorrhagic or oedematous or both. Frequently an oedematous condition of the connective tissues of the neck or about the trachea is very marked.

The muscles vary in color but usually they are darker than normal, and like the skin, they often become sprinkled with ecchymoses. The heart muscle suffers from parenchymatous changes (myocarditis).

In the larger cavities of the body, a sanguinolent fluid is found in moderate quantities. Blood extravasations of different sizes are seen under the serous membranes, particularly on the mesentery and mediastinum. The subserous connective 
tissue, especially on the mesentery, anterior cavity of the mediastinum, and in the neighborhood of the kidneys, is often infiltrated with a gelatinous substance. On this account the neighboring lymph glands are considerably swollen, filled with serum and sprinkled with hemorrhages. The internal organs contain a large quantity of blood. All the larger veins and the heart are filled with blood, while the surrounding tissues show sanious imbibition.

The spleen is considerably enlarged (two to five times its normal size), either uniformly or by prominent tumors. The pulp is soft, more or less fluid, and stained dark red. The capsule of the spleen is always very tense and is frequently extravasated with blood. Sometimes small raised vesicles are to be seen on its surface.

The liver and kidneys are highly congested and somewhat enlarged. The parenchyma contain areas of blood infiltration and the cells themselves manifest various kinds of degeneration. The portal lymph glands often appear enlarged, and the retroperitoneal tissue may be infiltrated with a serous, gelatinous fluid. The subperitoneal tissue of the intestines and of the abdominal walls may be similarly affected.

The nature of the lesions of the intestinal canal varies according as the disease is intestinal anthrax, or anthrax caused by inoculation. In cases of inoculation-anthrax, the intestine is frequently normal. In other cases there may be submucous and subserous hemorrhages, or swelling of the mesenteric glands. The principal changes in intestinal anthrax are always found in the small intestine, chiefly in the duodenum, more rarely in the colon. In the milder cases of intestinal anthrax the mucous membrane is affected, sometimes by circumscribed, or diffuse swellings. The specific bacteria are often found in very large numbers on the surface of the mucous membrane. Necroses and ulcers appear in those parts where the bacteria are most thickly congregated. In very severe cases, the abomasum or the first stomachs ny be affected with gelatinous and sanious infiltrations of the mucous membrane. The mucosa of the abomasum, and especially of the duodenum, is, in consequence of excessive hyperaemia, dark red or almost 
black, and is covered with erosions and ulcers or necroses which may extend down to the submucosa. The contents of the intestine are bloody, and the submucosa is infiltrated with a serous, gelatinous, or hemorrhagic exudate, so that the mucous membrane often projects, in the form of large tumors, into the lumen of the intestine. On the site of Peyer's patches and the solitary follicles we may find flat or prominent nodules, the surface of which are covered with diphtheritic crusts.

The lungs are greatly congested, oedematous and show areas of ecchymoses. The entire respiratory mucous membrane is considerably reddened and affected by ecchymoses. The mucous membrane of the pharynx and opening of the larynx is often so oedematous that stenosis of the larynx takes place. The contents of the trachea and the bronchi consist mostly of bloody froth or mucus.

The brain is often studded with ecchy moses. The surface of its membranes often exhibits hemorrhages with an accumulation of sanious serum in the ventricles. Extravasations of blood sometimes occur on the anterior chamber of the eye and under the retina. All the other organs show hemorrhages, and the urine frequently contains blood.

The blood is very dark, has a tarry or varnish like lustre, and shows little tendency to coagulate. It does not assume its normal red color when exposed to the air. The red blood corpuscles are more of less changed. The number of leucocytes is considerably increased.

The bodies of animals which have died from anthrax are often well nourished. Rigor mortis is absent and they decompose quickly. Very frequently blood flows from the natural openings of the body, and the rectum is sometimes prolapsed.

All the foregoing lesions may be absent in very acute apoplectic cases. The specific organism is, however, always present in the cadaver.

$\$ 79$. Differential diagnosis. It is important not to confuse anthrax with a number of non-specific disorders and accidental causes of death. The suddenness of the attack, and in very virulent cases, the short duration of the disease may tend 
to the mistaking of it for poisoning, cerebral apoplexy, pulmonary congestion, heat apoplexy, death from lightning, or acute gastro-intestinal inflammation. The affection known as corn stalk disease is not infrequently taken for anthrax and vice versa. In all of these cases the doubt following the postmortem can be easily settled by a bacteriological examination which, with genuine anthrax, will reveal the presence of Bacterium anthracis.

There are, however, certain specific diseases from which anthrax must be differentiated. The more important of these are symtomatic anthrax (black leg), malignant oedema, and septicaemia hemorrhagica. Rabies is not infrequently taken for anthrax. If the diagnosis cannot be determined by the anatomical changes (which can be relied upon only in somewhat typical cases) the positive diagnosis can be made only with the finding of the anthrax bacteria. In animals just dead, where decomposition has not begun, these organisms can usually be found in properly stained cover-glass preparations made directly from the blood or tissues. After decomposition begins to take place, a putrefactive organism, that is not easily distinguished from that of anthrax, often appears in the tissues. It is, therefore, necessary in such cases to resort to culture methods before a positive statement can be made. As the bacilli of malignant oedema and symptomatic anthrax are anaerobes, they will not develop in aërobic cultures such as on slant agar or in bouillon. The bacterium of septicaemia hemorrhagica being a small oval organism is easily told from that of anthrax. Bacillus subtilis has occasionally been taken for the anthrax bacterium; but it is readily separated morphologially and in cultures, especially in bouillon, where subtilis forms a firm, wrinkled membrane over the surface of the liquid which remains clear while the anthrax organisms grow in flocculi within the medium. It is important to recognize the possibility of error, if the conditions restrict the examination to the study of the microscopic preparations.

$\$ 80$. Protective inoculation. Toussaint was the first to make use of protective inoculations in anthrax. He. heated defibrinated anthrax blood to a temperature of 50 to $55^{\circ} \mathrm{C}$. for 
from $I_{5}$ to 20 minutes then injected it as a protective agent. Pasteur, however, was the first to prove that immunity could be obtained by the use of cultures of attenuated bacteria. Several methods of attenuating the specific organisms have been proposed by Pasteur, Toussaint, Chaveau, Chamberland, Arloing and others.

Pasteur's method consists in inoculating the animal with a small quantity of a culture which has been grown at a high temperature -42 to $43^{\circ} \mathrm{C}$. - for several days. This deprives the bacteria of their virulence. To strengthen the resistance, the animals are again inoculated with a stronger virus. After the two inoculations, they are said to be protected against the most virulent anthrax ; but the immunity is of short duration. Chamberland reported in 1894 that a total of $1,988,677$ animals had been treated by this method in France, and that the loss from anthrax had diminished from so per cent. in sheep and 5 per cent. in cattle to less than I per cent. Cope, in his report to the English Board of Agriculture, regards the conclusions of Chamberland as somewhat fallacious, because in order to prove that the animals inoculated received immunity, it should be shown that they were subsequently exposed to the risks of natural infection. The excellent work which has been done by Neal and Chester, at the Delaware College Experiment Station, has shown the possible efficiency of this method. Of the 33 I cows which they vaccinated against anthrax, two died of the disease, giving a death rate of less than I per cent, and this in a territory so saturated with the virus that it was practically impossible to keep cattle at all before its use.

A more critical study of the reports on the use of this vaccine shows that while success can not be denied, failures must be admitted. It is reported both in England and Germany that the Pasteur vaccine has not been a marked success. In England, Klein, who tested the vaccine used in that country, found that if the animals did not die from the effect of the vaccine, they did when exposed to the disease. The German veterinarians and agriculturalists agree that the first vaccine is mild and harmless, but that the second vaccine, even in the hands of experts, is dangerous and often fatal. 
The fact is reported to have been demonstrated by experiment that the virulence of the attenuated virus can be easily restored. Again, it has been shown by the investigations of Chester and Neal, of the Delaware College Agricultural Experiment Station, that a vaccine which succeeded at one time subsequently proved fatal. The vital objection to this method is, that it requires the use of the living bacteria which later may become virulent and consequently cause a subsequent outbreak. The scattering of pathogenic organisms, even in an attenuated condition, should be avoided, if possible. It must be admitted, however, that Pasteur's method has done much good and helped to rob anthrax of much of its former terror, especially for the farmers of Europe. In America the spread of anthrax has been checked in many districts by its use. Dalrymple has recently pointed out its success in the lower Mississippi valley. Notwithstanding, it is highly probable that the spreading of a knowledge of the specific cause of this disease with that of proper disposition of dead animals has also exerted much influence for good in checking its ravages.

In Germany and England the stamping-out system is considered superior to vaccination. According to Crookshank, in England it is regarded as the only reliable means of suppressing the disease. To this end rigid laws have been enacted. In this country as rigid measures as possible for its eradication seem infinitely better than the general adoption of methods for establishing a tolerance for its existence.

S 8r. Law's modification of Toussaint's method. Law has followed with marked success a modification of Toussaint's method. This consists in heating the blood taken from an animal just dead from anthrax or killed in the last stages of the disease, to the boiling point of water, grinding it in a little sterile water or bouillon and injecting the resulting liquid. The writer has employed this method in two outbreaks where immediate action was imperative. In one of the epizoötics where it was tried, the deaths had reached three a day and the astonishing result followed that not an animal died after the injection. In the second outbreak the blood injection produced 
no apparent diminution in the extent of the disease. It was followed with Pasteur's vaccine with very satisfactory results. It has not been demonstrated, however, that the sudden check in the spread of the disease was not a coincidence rather than the effect of the blood. A single experiment in cattle to test the efficiency of the heated blood in protecting against anthrax inoculation gave negative results. The same held true for experiments with guinea pigs. Loeffler has already pointed out the fact that small experimental aninuals are not readily immunized to anthrax.

The disposition of dead animals in an outbreak of anthrax is a matter of much importance. In all cases they should be burned if possible, if not, they should be buried deeply and thoroughly covered with quick lime before the dirt is replaced. The ground over the place where they are buried should be fenced in to prevent other animals from grazing over it and the surface should be hurned annually for some years to destroy spores should they be brought to the surface. In all cases the well animals should be removed from the barns or yards containing the sick ones and from pasture lands on which the sick became infected. The temperature of the supposedly healthy and uninfected animals should be taken morning and evening for from one to two weeks after they are removed and all of those showing an elevation of temperature should be isolated. By careful isolation and safe disposition of the dead animals the spread of the disease can be checked. Animals do not, as a rule, spread the virus when the first symptom (rise of temperature) can be detected. All infected stables and yards should be thoroughly disinfected.

\section{REFERENCES.}

I. Chester. Anthrax, bacteriological work. Report Del. Agr. Expt. Station. is95, p. 64.

2. Chester. Protective inoculation against anthrax. Proceedings of the Society for the Promotion of Agricultural Science. IS96, p. 52.

3. Davaine. Recherches sur les infusoires du sang dans la maladie connue sous le nom de sang de rate. Comp. Rend. de l'Acad. des. Sc. . 1863 . IS64. IS65.

4. DALRYMPLE. Anthrax and protective inoculation in Louisiana. Proceedings of the Am. Vet. Med. Assn. I901, p. 147. 
5. Koch, Die Aetiologie der Milzbrand-Krankheit begründet auf die Entwickelungsgeschichte des Bacillus Anthracis. Cohn's Beitr. zur Biol. der Pflanzen. Bd. II ( IS76) p. 277.

6. M'FadyenN. Anthrax. The Jour. of Comp. Path. and Therap. Vol. XI. ( IS9S) p. 5 I.

7. MoORE. Report of an outbreak of Anthrax. Annual Report, Commissioner of Agriculture of the State of New York. IS97.

S. PASTE, Ch, CHAMBERland ET Roux. De l'attenuation des virus et de leur retour à la virulence. Comp. Rend. de l'Acad. des Sc. T. XCII ( IS8I). p. 427.

9. PASTEUR. Le vaccin des charbon. Ibid. p. 666.

IO. POLLENDER. Mikroscopische und michrochemische Untersuchung der Milzbrandblutes. I $\$ 55$.

\section{INFECTIOUS LEUKAEMIA IN FOWLS.}

S 82. Characterization. A specific leukaemia of fowls caused by a bacterium. It is not known whether or not other species of domesticated birds are susceptible.

\$ 83. History. This disease was first briefly described, but not named, by Moore in I895. At that time it had been studied in but a few fowls and these the last to die in their respective flocks. In the following year other fowls were examined very carefully from two outbreaks of the disease and it is upon the data obtained in these investigations together with those procured from many produced cases that the description of the disease is based. In 1898 , Dawson found it to be the cause of very serious losses among poultry near Baltimore, MI. In all of the outbreaks studied, the owners of the fowls first reported the disease as chicken cholera.

\$ 84. Geographical distribution. It was first studied in fowls taken from an outbreak in Virgiuia. Since then, it has been identified in Maryland, the District of Columbia, and Block Island, R. I. There is good evidence in the numerous reports of destructive fowl disease to believe that it is quite widespread in the United States. Thus far, there seem to be no reports of its existence in other countries. 
$\$ 85$. Etiology. Moore isolated and described a pathogenic bacterium which he designated Bacterium sanguinarium. With this organism the disease has been produced in healthy fowls both by feeding cultures and by intravenous injections. Its etiological relation to the disease is, therefore, quite clearly established. It is possible that certain accompanying conditions may be necessary in conjunction with the organism to cause

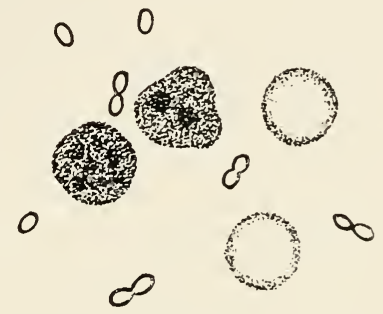

FIG. I3. Bacterium sanguinarium. the disease to spread rapidly in a flock. Experimentally it did not spread from diseased (inoculated or fed) to healthy fowls when kept in the same yard.

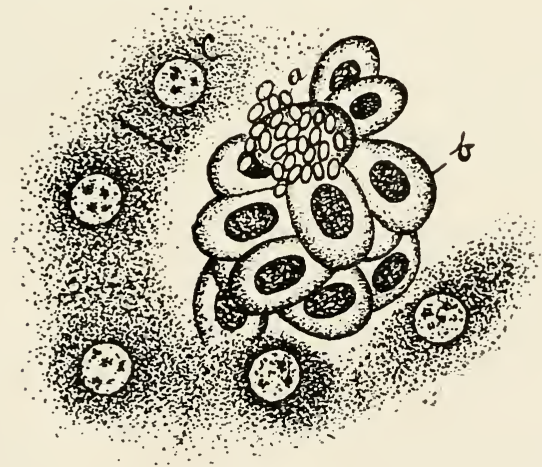

FIG. I4. A clump of Bact. sanguinarium in a blood space in the tiver. (a) Bacteria, (b) red blood corpuscles, (c) liver cells (much enlarged).

\$86. Symptoms. From the statement of the owners of the diseased fowls in the different outbreaks and from the appearance of those in which the disease was artificially pro- 
duced, little can be positively stated concerning the early symptoms. There is a pronounced anæmic condition of the mucosa of the head. An examination of the blood shows a marked diminution in the number of red corpuscles and an increase in the number of white ones. In the disease produced artificially by feeding cultures of the specific organism there is, in most cases, a marked drowsiness and general debility manifested from one to four days before death occurs. The period during which the prostration continues varies from a few hours to two days. The mucous membranes and skin about the head become pale. There is an elevation of from I to 4 degrees of temperature. The fever is of a continuous type, as shown in the appended temperature chart of two fowls in which the disease was produced artificially :

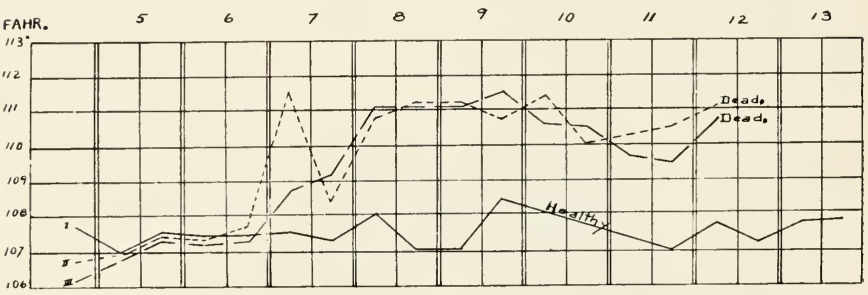

FIG. 15. Tempcrature chart of two cases of infectious leukaemia artificially produced in fowls.

Although the course of the disease in different fowls is usually constant, there are many variations. The time required for fatal results is from two to three weeks, but ordinarily death occur in about eight days after feeding the cultures. The rise in temperature can be detected about the third day and external symptoms about the fifth or sixth, occasionally not until a few hours before death. The symptoms observed in the cases produced by feeding correspond with those described by the owners of affected flocks.

As indicated in the inoculation experiments, the symptoms following the intravenous injection of the virus were, as 
would be expected, considerably modified from those in fowls which contracted the disease by the ingestion of cultures of the specific bacterium.

$\$ 87$. Morbid anatomy. The only constant lesions found in the fowls which contract the disease naturally, as well as in those fed upon the virus, are in the liver and blood. The liver is somewhat enlarged and dark colored. A close inspection shows the surface to be sprinkled with minute grayish areas. The microscopic examination shows the blood spaces to be distended. The hepatic cells are frequently changed, so that they stain very feebly, and not infrequently the cells are isolated and their outlines indistinct. Occasionally foci are observed in which the liver cells appear to be dead and the intervening spaces infiltrated with round cells. The changes in the hepatic tissue are presumably secondary to the engorgement of the organ with blood.

The rareness with which the intestinal tract is affected in both the natural and artificially produced cases is exceedingly interesting and important for the differential diagnosis. There is in most cases a hyperæmia of the nucous membrane of the colon, but this condition is not uncommon in the healthy individual. The kidneys are generally but not uniformly pale. They are streaked with reddish lines, due to the injection of blood vessels. In section the tubular epithelium appears to be normal. The kidneys seem to be, from the number of bacteria in the cover-glass preparations, especially favorable for the localization of the specific organism. The spleen is rarely discolored or engorged with blood. The lymphatic glands are not appreciably enlarged in any individual examined. The lungs except in chronic cases are normal. The heart muscle is usually pale and sprinkled with grayish points, due to cell infiltration and necrosis. These lesions are so common that it seems safe to consider them characteristic manifestations. Death usually occurrs in systole, the auricles containing very little thin, unclotted blood. The brain and spinal cord are unaffected.

The most important alterations are found in the blood. These consist of the gradual disappearance of the red cor- 
puscles and increase in the number of white ones, as determined by blood counts made daily or every other day, from the time of inoculation or of feeding the virus until the day of death.

The diminution in the number of red corpuscles and the increase in the number of white ones are illustrated in the blood count of two cases of artificially produced disease. (p. I05).

In carefully heated cover-glass preparations of healthy fowl blood stained with methylene-blue and eosin, the nuclei are colored a deep blue, and the cellular protoplasm surrounding the nucleus is stained by the eosin. In similar preparations made from the blood of the affected fowls there are a

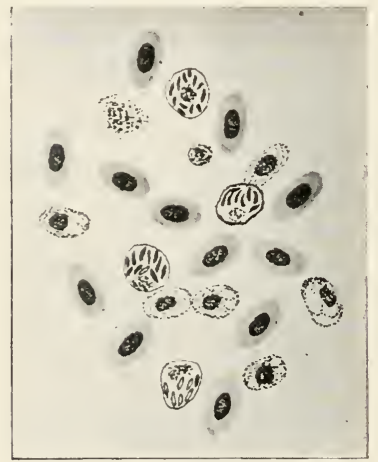

FIG. 16. Blood from a well advanced case of infectious leukaemia showing changes in red corpuscles and increase in number of leucocytes. greater or less number of corpuscles which do not take the eosin stain. In these the portion of the corpuscle surrounding the nucleus remains unstained or becomes slightly tinted with blue. It occasionally contains one or more vacuoles, and the margin is frequently broken. In some instances a considerable portion of the corpuscle has disappeared, leaving a few free nuclei. The apparent dissolving away of the red corpuscles has been frequently observed, and corpuscles showing the intermediate stages are readily detected in carefully prepared specimens.

The cause of the destruction of the red corpuscles is not yet satisfactorily explained. In his report on fowl cholera, Salmon illustrates leucocytes surrounding the red corpuscles, but the marked diminution of the red cells was not determined. He speaks, however, of the pale color of the blood. In 
fresh preparations of the blood, portions of red cells may be seen within the granular leucocytes. The determination of the extent of this mode of destruction of the red corpuscles necessitates further investigation.

TABLE SHOWING CHANGES IN THE NUMBER OF CORPUSCLES.

Fowl No. 82, inoculated in the wing vein, February 6.

\begin{tabular}{|c|c|c|c|c|}
\hline Date & $\begin{array}{l}\text { Tempera- } \\
\text { ture }\left({ }^{\circ} \mathrm{F} .\right)\end{array}$ & $\begin{array}{l}\text { Number of } \\
\text { red cor- } \\
\text { puscles } \\
\text { per c. mm. }\end{array}$ & $\begin{array}{l}\text { Number of } \\
\text { white cor- } \\
\text { puscles } \\
\text { perc. mm. }\end{array}$ & Remarks \\
\hline Feb. 6 & $\begin{array}{l}107.4 \\
109 \\
108.2 \\
108.4 \\
107.4 \\
110.2 \\
108\end{array}$ & $\begin{array}{l}3,744,444 \\
3,417,391 \\
2,784,700 \\
2,807,692 \\
3,4,1,618 \\
2,133,333 \\
2,530,000\end{array}$ & $\begin{array}{r}21,222 \\
26,057 \\
55,000 \\
76,925 \\
90,909 \\
100,000 \\
140,000\end{array}$ & $\begin{array}{l}\text { Well. } \\
\text { Apparently well. } \\
\text { Do. } \\
\text { Do. } \\
\text { Feathers ruffled ; refuses food. } \\
\text { Very quiet; comb pale. } \\
\text { Fowldied later in the day. }\end{array}$ \\
\hline \multicolumn{5}{|c|}{ Fowl No. 5or, fed culture March 26.} \\
\hline Date & $\begin{array}{l}\text { Tempera- } \\
\left.\text { ture ( }{ }^{\circ} \mathrm{F} .\right)\end{array}$ & $\begin{array}{l}\text { Number of } \\
\text { red cor- } \\
\text { puscles } \\
\text { per c. mm. }\end{array}$ & $\begin{array}{l}\text { Number of } \\
\text { white cor- } \\
\text { puscles } \\
\text { per c, mm. }\end{array}$ & Remarks. \\
\hline $\begin{array}{r}\text { Mar. } 26 \\
25 \\
\text { Apr. } 2 \\
3 \\
4\end{array}$ & $\begin{array}{l}106.2 \\
110 \\
110.6 \\
106\end{array}$ & $\begin{array}{l}3,534,000 \\
2,430,000 \\
1,694,210 \\
1,745,000\end{array}$ & $\begin{array}{r}18,940 \\
70,000 \\
80,000 \\
245,432\end{array}$ & $\begin{array}{l}\text { Well. } \\
\text { Fowi eats very little. } \\
\text { Blood very pale: fowl weak : refuses food. } \\
\text { Very weak: many red corpuscles at- } \\
\text { tacked hy leucocytes. } \\
\text { Found dead. }\end{array}$ \\
\hline
\end{tabular}

In fresh preparations of the blood of affected fowls examined in 'Toisson's fluid, red corpuscles which take the riolet stain more or less intensely throughout are frequently observed.

In the blood of poultry two distinct classes of white corpuscles are conspicuous. The first which predominates in numbers, contains from one to four nuclei, and the cytoplasm is sprinkled with a variable number of round, elongated, or spindle-shaped bodies. In the fresh condition they are showing engorgement of blood.

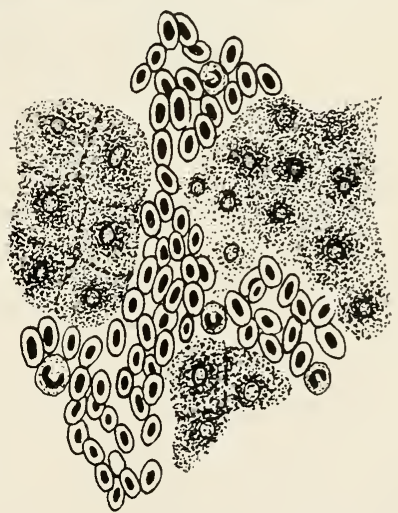

FIG 17. Section of chicken's liver- 
highly refractory. They stain with eosin, and if the preparations are heated sufficiently they will retain certain of the aniline dyes. The other class consists of round or nearly round cells which take the blue stain feebly. Usually it is difficult to detect the nucleus, although it is occasionally distinct. Between these two types there are many varieties. The leucocytes containing the spindle-shaped bodies appear to be the phagocytes as they were the only ones which were observed to attack the red corpuscles. Bacteria have not been demonstrated in these cells, although their presence has, in several cases, been suspected. From the appearances observed in the red blood corpuscles it

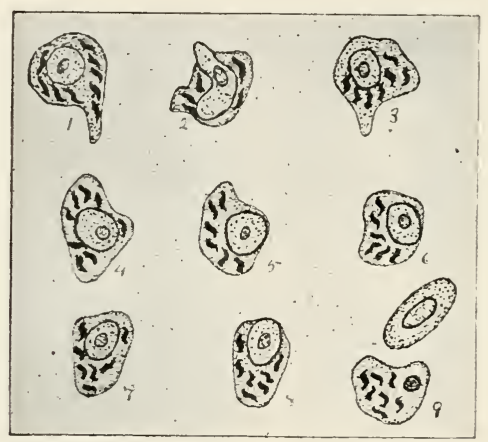

FIG. IS. The phagocytic action of the white blood corpuscles upon the red ones in case of advanced leukaemia. The changes represented from I tog took place in 35 minutes.

seems highly probable that phagocytosis plays a comparatively large part in their destruction. Another hypothesis is also suggested, namely, that a toxin produced during the multiplication of the specific organism has this effect on the red corpuscles. In the fresh preparations we can observe the phagocytes attacking the red cells. In the stained ones mutilated red corpuscles and free nuclei are present. The hypothesis is suggested that the leucocytes partially digest certain of the red corpuscles in their attack upon them. Whether these changes are entirely attributable to the phagocytes is still an open question. 
In the blood from healthy fowls it is comparatively rare to see one of the white corpuscles attacking a red one. As the disease progresses, however, this warfare becomes very conspicuous, owing perhaps to the increased number of the colorless cells. Up to the present the study of these corpuscles has not been extended beyond the observation of the general appearance of these structures, and no attempt is made to explain the apparently marvelous increase in the number of the lencocytes. It is an interesting and as yet unexplained fact that the increase in the white corpuscles is apparently restricted to those containing the spindle-shaped bodies.

\$ 88. Differential diagnosis. Intestinal disturbances, especially diarrhoea and fowl cholera, are the diseases liable to be mistaken for infectious leukaemia.

A comparison of the important changes in the morbid anatomy in fowl cholera as described by European writers and in the disease under consideration can be made from the appended columns, in which their more characteristic lesions are contrasted :

\section{Lesions in Fowl cholera.}

1. Duration of the disease from a few hours to several days.

2. Elevation of temperature.

3. Diarrhoea.

4. Intestines deeply reddened.

5. Intestinal contents liquid, mucopurulent, or blood stained.

6. Heart dotted with ecchymoses.

7. Lungs affected, hyperæmic or pneumonic.

8. Specific organisms appear in large numbers in the blood and organs.

9. Blood pale (cause not determined).

Io. Condition of leucocytes not determined.
Lesions in infectious leukaemia. "?

I. Duration of the disease from a few hours to several days.

2. Elevation of temperature.

3. Diarrhoea very rare.

4. Intestines pale.

5. Intestinal contents normal in consistency.

6. Heart usually pale and dotted with grayish points, due to cell infiltration.

7. Lungs normal, excepting in modified cases.

8. Specific organisms comparatively few in the blood and organs.

.9 Blood pale, marked diminution in the number of red corpuscles.

10. Increase in the number of leucocytes. 
Attention should be called to the fact that as yet there seems not to have been a careful study of the condition of the blood in fowl cholera. Salmon observed many changes in this fluid which may have been similar to or identical with those herein recorded.

The difference between the specific organism of these two diseases can be readily appreciated by a comparison of the more diagnostic properties of each ; they are arranged in parallel columns, as follows :

Bacterium of fowl cholera.

I. Bacterium short, with oval ends.

2. It usually appears singly in tissues.

3. Ordinarily it exhibits a polar stain. (From tissue.)

4. Grows feebly or not at all on gelatin.

5. It does not change inilk.

6. Resists drying from one to three days.

7. Kills rabbits inoculated subcutaneously in from eighteen to twenty-four hours.

8. It kills fowls when injected subcutaneously in small quantities.

\section{Bacterium sanguinarium.}

I. Bacterium short, with ends oval or more pointed.

2. It usually appears in pairs united end to end or in clumps in tissues.

3. It gives a light center, with uniformly stained periphery. (From tissue.) Rarely a polar stain is observable.

4. Decided growth on alkaline gelatin.

5. Saponifies milk.

6. Resists drying from eight to twelve days.

7. Kills rabbits inoculated intravenously in from three to five days. Rabbits inoculated subcutaneously remain well or die in from six to ten days.

8. It does not kill fowls when injected subcutaneously in small quantities.

While there are many similarities in the symptomatology of these two diseases, there are pronounced differences in the morbid anatomy and in the specific microorganisms. These facts will render the positive differentiation dependent upon a careful bacteriologic and pathologic examination. In fowl cholera the course of the disease is more rapid than in leukæmia. 


\section{REFERENCES.}

I. DAwsox. Infectious leukaemia. Annual Report of the Bureau of Animal Industry, U.S. Dept. Agric. isgs.

2. MOORE. A study of a bacillus obtained from three outbreaks of fowl cholera Bulletin Vo. 8, U. S. Bureau of Animal Industry. 1894.

3. MOORE. Infectious leukaemia in fowls-A bacterial disease frequently mistaken for fowl cholera. Annual Report of the Bureau of Animal İndustry. ${ }_{1}$ S95-6.

\section{SIINE ERYSIPELAS.}

\$ 89. Characterization. This disease, peculiar to swine, is determined by a rise of temperature, cerebral disturbances, and pronounced reddening of areas of the skin. It is a disease of adult life. It is stated that pigs are rarely attacked under three months or over three years of age. Lydtin and Schottelius found some differences in the degree of susceptibility of certain breeds of swine. The common country pig was least susceptible. It is known in France as rouget and in Germany as Rothlauf.

s go. History. This disease has been known in Europe for many years. It has not been positively identified in the United States. Smith found a bacterium in rabbits inoculated with the organs of pigs that had died of an undetermined disease in Minnesota, which was either the bacterium of swine erysipelas or of mouse septicaemia. The latter organism had been recorded on two previous occasions from pigs in this country.

9I. Geographical distribution. Swine erysipelas is an infectious disease which occurs enzoötically and in epizoötics in most of the countries of Europe. Like anthrax, erysipelas is often stationary. It was formerly restricted in Bavaria to the districts along the Danube, and was entirely unknown in southern Bavaria (Kitt). It is stated that the disease tends to become enzoötic chiefly in valleys and lowlying plains which have slow-flowing streams, and heavy, damp, clay soil ; and that sandy and granite soils are comparatively free from it. It occurs chiefly during the months of July, August and September, although it appears sporadically during the winter months. 
92. Etiology. Loeffler and Schütr pointed out in 1885 that swine erysipelas was caused by a very slender bacterium i to $2 \mu$ long and 0.3 to $0.4 \mu$ broad, straight or slightly curved, ends not rounded and in cultures often appearing in filaments. It is very closely related to the bacterium of mouse

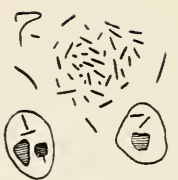

FIG. 19. Bacsepticaemia described by Koch in 1878 . In erysipelas. Europe where the bacterium of mouse septicaemia is quite common swine erysipelas prevails. In this country the bacterium of swine erysipelas has not been positively identified. At three different times an organism resembling it has been isolated from swine. There is much uncertainty concerning the relationship of the bacterium of mouse septicaemia to that of this disease. Smith has suggested that possibly the bacterium which has been found in this country may gain virulence sufficient to produce epizoötics, if such is not already the case. It is exceedingly important that careful search be made for this organism in the outbreaks among swine where the nature of the disease is not clearly determined. House mice and pigeons are susceptible to the bacteria of swine erysipelas Guinea-pigs and fowls are immune. Rabbits suffer from erysipelatous swellings when inoculated subcutaneously in the ear.

$\$ 93$. Symptoms. It is stated that after a period of incubation of at least three days, the disease usually begins suddenly and violently. The animal refuses food, makes efforts to vomit, has a rise of temperature, manifests severe nervous disturbance, is very weak, torpid and indifferent to its surroundings. When approached it tries to hide itself under its bedding. The hind quarters become weak and paralyzed. Muscular spasms and grinding of teeth are sometimes observed. At first there is constipation, the conjunctiva is of a dark red or brownish-red color, and the eyelids are sometimes swollen. Usually a day or two after the first symptoms develop or, perhaps, from the first attack, reddish spots appear on the thin parts of skin, such as the region of the navel, lower surface of the chest, perineum, inner surface of the thighs, ears and throat. These spots, which at first are bright red and about 
the size of a man's hand, become, later on, dark red or purple, and soon unite into large irregularly-shaped patches. As a rule, they are neither painful to the touch or prominent, but sometimes they show a slight inflammatory swelling. The skin of the red spots, especially of the ears, may suffer from an eruption of vesicles and may even slough. The reddening of the skin may be very slight in severe cases, or may appear only immediately before, or even after death. Death takes place usually on the third or fourth day. In the very severe form, the animal may die in twenty-four hours, otherwise the disease requires a week or longer to run its course.

Jensen considers that this disease, instead of being uniform in its clinical aspect, manifests itself in the following forms, which differ from each other by well-marked peculiarities. He also maintains that there may sometimes be transitional forms between the respective varieties which lie enumerates. The forms recognized as varieties of this disease but more generally considered as distinct maladies and known by different names are as follows : (1) true erysipelas, (2) swine urticaria, (3) erysipelas without redness of the skin, (4) diffuse necrotic erysipelas of the skin, (5) endocarditis of erysipelas. Different forms of epizoötic erysipelas have also been described by Cornevin, Hess and others.

$\$ 94$. Morbid anatomy. In the ordinary form of epizoötic erysipelas there is a septicaemic condition without any well marked morbid changes of separate organs. In less acute cases the septicaemia may give way to haemorrhagic and diphtheritic gastro-enteritis considerable swelling of the $1 \mathrm{ym}$ phatic system, haemorrhagic or parenchymatous nephritis and hepatitis, acute swelling of the spleen, and myositis. The hemorrhagic gastro-enteritis consists at first of excessive inflammation of the mucous membrane of the stomach in the region of the fundus. The mucosa shows a darkred discoloration, which is partly diffuse and partly in spots. The cells suffer from cloudy swelling and the mucous membrane is covered with a viscid layer of mucus and may have superficial scabs. The intestinal mucous membrane is swollen, especially on the top of the folds and in the neighbor- 
hood of Peyer's patches. It is infiltrated with blood and sometimes shows superficial scabs. Less frequently, circumscribed parts of the mucosa of the caecum and of the anterior parts of the colon suffer from a diptheritic affection.

The solitary follicles and Peyer's patches appear as prominent raised patches. Sometimes they are infiltrated with blood and surrounded by a reddish band. There is ulceration and cicatrisation of the solitary and agminated follicles. The mesenteric glands become more swollen than the other glands of the body, of a dark red color, and show softening. The surface of fresh sections is dun-colored with interspersed darkred areas. The paraglandular tissue is hyperaemic, and infiltrated with blood.

The kidneys are enlarged, the cortex of a grayish-red and the medullary portion of a very dark-red color. Frequently catarrhal nephritis occurs as a complication.

The acute swelling of the spleen arises in consequence of an acute hyperaemia with an increase of the cellular constituents of the spleen in which case the organ is enlarged, but not softened as in anthrax. The pulp is of a purple color, moderately soft and free from haemorrhages.

There is cloudy swelling and enlargement of the liver. The surface of sections has a greyish-brown color, and the acini are widened. The nuscles are grey in color, soft, flaccid, watery, glistening and sometimes they are sprinkled with haemorrhages. They give the general appearance of boiled flesh. The myocardium shows similar spotted changes, and haemorrhages beneath the endocardium.

In the abdominal and thoracic cavities and pericardium, there may be found small quantities of an orange-colored, clear fluid, which may be mixed with flaky coagula. Punctiform hæmorrhages often appear under the serous nembranes, especially on the auricles. Many English veterinarians regard the occurence of more or less luxuriant vegetations on the valves of the heart to be so frequent as to be almost diagnostic. It would appear from the literature that this endocarditis is not nearly so common in continental Europe. Severe haemorrhages in the brain and in the dorsal and lumbar portions of 
the spinal cord are rare. The lungs remain unchanged, or at most exhibit a post mortem oedema. By nicroscopical examination, the specific bacteria are found everywhere in the body, especially in the spleen and kidneys, and to a less extent in the blood.

\$95. Differential diagnosis. Swine erysipelas is to be differentiated from (I) hog cholera and swine plague. The frequent reddening of the skin in these diseases together with the modified lesions so frequently observed, may cause confusion. The bacteriological examination will enable the positive diagnosis to be made. (See hog cholera and swine plague.)

(2) From anthrax which is very rare in swine. Here, too, the bacteriological examination discloses the true nature of the disease.

(3) Erythemata due to various dietary causes.

The significance of a deep reddening of the skin about the head, abdomen and thighs of pigs is not fully determined. It is clear, however, that such a condition often occurs in the absence, so far as present knowledge goes, of a specific infection.

\$ 96 . Preventive inoculation. Pasteur's protective inoculation is at present the chief prophylactic means employed against epizoötic erysipelas. The exact method of preparing the inoculation material has not been published. It is known that Pasteur's vaccine is obtained by passing the bacteria through several generations of rabbits in succession and then cultivating them in nutritive fluid. The animals inoculated after Pasteur's method become, according to him, only slightly ill, and acquire immunity to erysipelas. The method of inoculation is as follows: the pigs at the age of nine to sixteen weeks are first injected in the subcutaneous connective tissue with a weak vaccine and in ten or twelve days after with a stronger vaccine.

The results of the practical application of this method show that it immunizes to a certain extent the inoculated animals. However, the risk of the treatment is said not to be insignificant. It is held that the inoculated pigs infect the healthy and may thus spread the disease. Besides, the adoption of this system renders the inoculated animals seriously ill, 
and according to various European reports, is attended by a mortality of about 4 per cent which it is affirmed exceeds the total loss caused by epizoötic erysipelas when allowed to run its course unmolested.

Metschnikoff found that the blood of immunized rabbits was antitoxic, and Lorenz maintains that the serum of swine which have recovered from swine erysipelas is also antitoxic, and will produce immunity in other animals. The treatment introduced by Lorenz is to inject the immunizing serum in the proportion of I cc. to every 10 kilogrammes of the body weight of the animal. Two days afterward 0.5 to I. o cc. of virulent culture is injected, and after twelve days the dose is doubled. Lorenz inoculated 294 pigs ; twelve were suffering from swine erysipelas, and of these 6 recovered and 6 died.

\section{REFERENCES.}

1. BANG. Teber Rothlauf-Endocarditis bei Schweinen. Deutsche Zeitschr. f. Thiermed. Bd. XVIII. (1Sgi). S. 27.

2. JENSEN Die Aetiologie des Nesselfiebers und der diffusen Hautnekrose des Schweines. Deutsche Zeitschr. f. Thiermed. (1S92). S. $27 \mathrm{~S}$.

3. LOEFFrER. Experimentelle Entersuchungen über SchweineRothlauf Arbeiten aus d. Kaiserichen Gesundheitsante. Bd. I. ( $\mathrm{SS}_{5}$ ). S. 46.

4. Lorfiz. Die Schutzimfung gegen Schweinerothlauf mit A11wendung eines aus Blutserum immunisirter Thiere hergestellten Impfstoffes. Deutsche Zeitschr. f. Thicrmed. Bd. XX. (1894). S. 1.

5. LORENZ. Die Veterinärpolizeiliche Behandlung des Schweinerothlaufes und die Schutzimfung. Berliner thierarz. Wochen. ( IS97). p. 574 .

6. Lorenz. Schutzimfungen gegen den Rothlauf der Schweine. Ibid. (1897). S. Iog.

7. MOORE. Mouse septicæmia bacilli in a pigs spleen with some observations on their pathogenic properties. Jour. of comp. Med. and Vet. Archives. Vol. XIII. (IS92). p. 333.

S. PASTUER ET Thuiliter. La vaccination du rouget des porcs á l'aide du virus mortel attenuè de cette maladie. Comptes Reudus Acad. des Sciences Vol. XCVII, iSS3. p. 1163 .

9. Scrütz. Ueber den Rothlauf der Schweine und die Impfung mit demselben. Arbeiten a.d. Kaiserlichen Gestendheitsamte. Bd. I. $\left(\mathrm{ISS}_{5}\right)$. S. 56.

Io. Sмiтн. An Examination of Pasteur's Vaccine for Rouget. Anmual Report U. S. Bureau of Animal Industry. ISS5. p. IS7.

11. SurTH. Swine erysipelas or mouse septicaemia bacilli from an outbreak of swine disease. Anmual Report, U. S. Bureau of Animal Industry. I $895^{-96 .}$ p. 166. 
GLANDERS.

$\$ 97$. Characterization. Glanders is one of the most important diseases of horses, asses and mules and when transmitted to man, one of the most fatal diseases of the human species. It runs an acute or chronic course attacking the lymphatic system more especially in the upper air passages, lungs, or skin. The disease is characterized by a strong tendency to the formation of small neoplasms or nodules which are likely to degenerate into ulcers from which exude a peculiar sticky discharge. In the very acute cases a considerable rise of temperature and general debility may accompany the formation of the lesions. Glanders of the skin is known as farcy. It is known in Germany as Rotz or Rotzkrankheit and in France as morve.

By direct inoculation several species of animals may be infected. Thus the disease has been reported in goats, rabbits, sheep, guinea pigs, field mice, and several of the wild animals, especially those of the cat tribe. Swine and pigeons are very slightly susceptible. Cattle, white mice, rats and domestic fowls seem to be immune.

5 98. History. The theory of the contagiousness of glanders was much doubted at the beginning of this century. The view taken at the Alfort Veterinary College was that glanders might arise spontaneously from an attack of strangles. This view was far more widely accepted than the theory of its contagiousness, which was stoutly supported by the Veterinary College of Lyons. It was not until Rayer ( 1837 ) had demonstrated the transmissibility of glanders to man, and Chaveau ( 1868 ) had shown that the virus was contained chiefly in the firm component parts of the infective material, that the fact of the infectious nature of the disease was accepted.

The theory of the spontaneous origin of glanders was widely accepted in Germany. Sixty years ago it was believed that glanders could be produced by the injection of pus, and that strangles could develop into glanders. Glanders was looked upon as a tubercular disease, scrofula, pyaemia, diphtheritis, general dyscrasia, and cachexia, respectively. Virchow was 
the first to declare that the nodules of glanders were independent, anatomical formations, which he placed under the heading of granulation tumors. Gerlach was the strong adrocate for the exclusively infectious origin of the disease. Leisering appears to have been the first to give an accurate description of the lesions.

The first biological researches into its nature were made in I 868 by Zurn and Hallier, who found a fungus which they believed to be the cause of the disease. In I882, Löffler and Schütz succeeded in finding the bacterium of glanders, in cultivating it, and in transmitting the disease to other animals by inoculating them with pure cultures of this organism. Their researches furnished the positive proof that glanders is a specific, infectious disease, produced exclusively by Bacterium mallei.

S99. Geographical distribution. Glanders exists in the greater part of the civilized world. It is more common in the temperate zones, where traffic in horses is active. In the United States it was largely confined to the Northern States before I861, but it spread over the South in connection with the civil war. It is said to have entered Mexico with the American cavalry in 1847 . Similarly, Portugal is said to have been exempt until the invasion by Napoleon in 1797 . Central Hindoostan was said to be free from it until the war with Afghanistan in 1878 . In all these cases, the movements of cavalry, artillery and of commissary trains were responsible for the introduction of the disease into new territory. In our own case the sale of horses and mules at the close of the civil war produced a very general diffusion of this disease from which the country is still suffering.

Insular places, especially if far from the main land and free from importation of horses, usually escape. Thus glanders is very rare in Iceland and in the Faroe islands. In Australia, Tasmania and New Zealand it is reported to be unknown. 
\$ roo. Etiology. Bacterium mallei, the specific cause of glanders, was discovered and isolated in pure culture almost at the same time (1882) by Loeffler, Schütz, Israel, Bouchard, Charrin, Weichselbaum, Kauzfeld, and Kitt. It is found in the recent nodules, in the discharge from the nostrils, pus from the specific ulcers, and

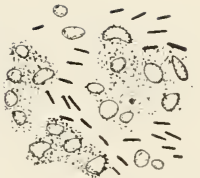

FIG. 20. Bacterium mallei. occasionally in the blood of animals affected with glanders.

Morphologically it is a small organism with rounded or pointed ends. It varies in breadth from $0.25 \mu$ to $0.4 \mu$ and from $1.5 \mu$ to $3 \mu$ in length. It is usually single but pairs, and long filaments, especially on potato cultures, are not rare. It frequently breaks up into short, almost coccus-like elements.

It stains with some difficulty. Of the aniline colors, the best results are obtained when the aqueous solutions of these dyes when they are made feebly alkaline. It is decolorized by Gram's method.

It grows well, but slowly at the body temperature on glycerine agar, in acid-glycerinized bouillon, on blood-serum and on potato.

Of the test animals, guinea-pigs and field mice are the most susceptible. In guinea-pigs, subcutaneous injections are followed in four or five days by swelling at the point of inoculation and sloughing of the skin which is followed by the formation of a chronic, purulent ulcer. The lymphatic glands become inflamed and symptoms of general infection develop in from two to four weeks; the glands suppurate and in males the testicles are involved. A purulent inflammation of the joints may occur. The formation of the specific ulcers upon the nasal mucous membrane, which forms one of the characters of the disease in the horse, rarely occur in the guinea-pig as a result of inoculation. The disease is often prolonged for several weeks or months. Guinea-pigs succumb usually in from eight to ten days when injected into the peritoneal cavity. In males, the testicles are invariably affected.

S ror. Symptoms. (a) Acute glanders. Acute glanders is common in the ass and mule, but infrequent in the 
horse. After a period of incubation of from three to five days the animal has a chill, elevation of temperature, a profuse muco-purulent sticky discharge, sometimes mixed with blood, from the nose. Particles of food arrested in the pharynx occasionally appear in the nasal discharge. If unilateral the margin of the nostril swells, the mucosa is dark-red, infltrated, marked with pea-like, yellowish elevations with red areolæ, which in a few days become eroded, thus forming spreading ulcers. The submaxillary lymphatic glands on the affected side become enlarged. There may, however, be a uniform swelling filling the whole intermaxillary space. The course is rapid and death ensues, usually from suffocation, in from the sixth to the fifteenth day. The acute form rarely if ever becomes chronic.

(b). Chronic glanders. In the horse, this form of the disease may begin with a chill but usually the onset is very insidious. There may be a muco-purulent, sticky discharge sometimes streaked with blood from one or both nostrils. There may be intermittent or continued lameness, arthritis, oedema of a limb, swelling of a testicle, cough, or epistaxis. There is ustrally a nodular but comparatively painless swelling of the submaxillary $1 \mathrm{ymph}$ gland, on the affected side. On palpation the swelling imparts a sensation suggestive of a number of peas. They are adherent to the adjacent structures. The nasal mucosa is congested, of a dark reddish color and sprinkled with superficial or deep ulcers either clean or covered with crusts.

Rarely the submaxillary glands only are apparently diseased. In other cases, there is only a cough, the latent lesions being confined to the lungs. In still other cases, the lesions are restricted to one or both testicles, the spleen, or some other internal organ, and symptoms may or may not be present. Chronic glanders frequently terminates in the acute form.

In chronic cutaneous glanders, with or without oedema of the limbs, there may be one or many nodules on the fetlock, or elsewhere on the line of the lymphatic vessels, with induration of the lymphatics extending from it. The nodules may be suppurating and discharging, or they may be closed. 
I02. Morbid anatomy. In chronic glanders, the most frequent locations of the lesions are on the respiratory mucous nembrane, in the lungs, lymph glands and skin. Other organs are more rarely invaded. The upper respiratory mucous membrane is the usual seat of the lesions. Glanders occurs in two forms, viz., (a) as circumscribed nodules with the formation of ulcers and cicatrices; and $(b)$ as diffuse or infiltrated lesions.

(a) In nodular glanders, which is the common form, the lesions are most frequently situated on the upper portion of the nasal septum, and in the cavities of the turbinated bones. The affection begins with the appearance of nodules varying in size from a grain of sand to a millet seed. They are more or less translucent, of a roundish or oval shape, and of a dirty gray or grayish red color. The nodules, which may attain to the maximum size of a pea, project somewhat abore the surface of the mucous membrane. They are surrounded by a reddish ring. Some of them are isolated and others are arranged in groups. Microscopically they consist of a large number of lymphoid cells, which disintegrate in the centre of the nodule. In consequence of the central fatty and purulent disintegration, the nodules become yellowish in color, discharge and form ulcers. These ulcers are sometimes superficial, sometimes deep, lenticular or crateriform, surrounded by a hard, indurated edge, and frequently becoming confluent with irregularly serrated and eroded edges. They are sometimes corered with a brownish crust. The ulcers may increase in area or in depth and may even involve the underlying cartilage or bone, causing perforation of the septum nasi, and distensions of the maxillary or exostoses on the turbinated bones. The shallow lenticular ulcers may heal withont leaving any visible changes; but the deeper ones, after granulating, leave a radiating, star-shaped cicatrix which is either smooth or horny, and which, according to the shape of the ulcer, may be of an irregular, oblong form. The nasal septum is frequently covered with these scars. The ulcers and cicatrices are sometimes found in the maxillary and frontal sinuses, in the guttural pouches and in the eustachian tubes. They may also occur in the larynx, especially in the re- 


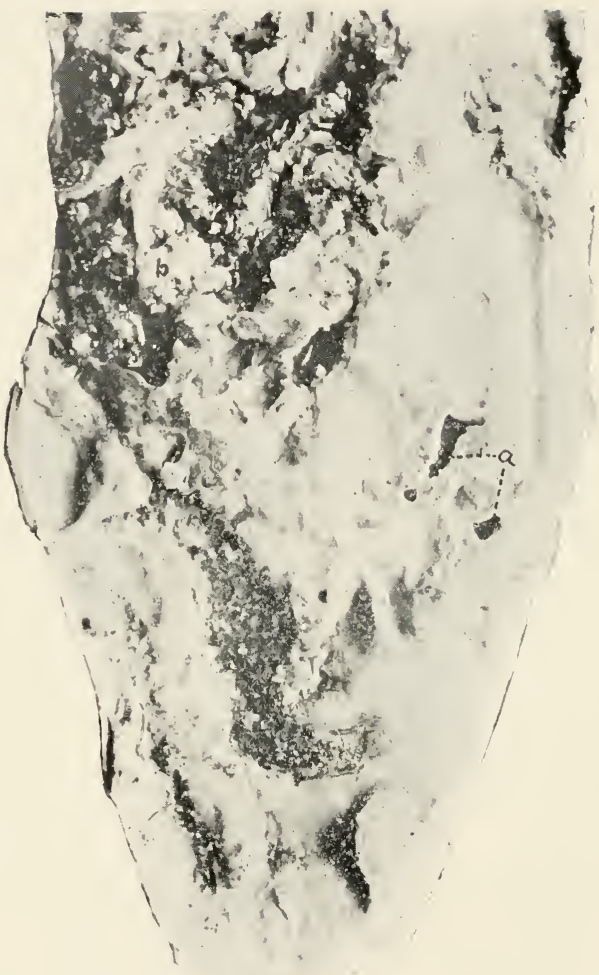

Fig. 2I. Photograph of a portion of a nasal septum showing ulcers in advanced glanders. (a) Perforations of septum.

gion of the lower vocal chords. In the trachea and even in the bronchi, particularly on the anterior surface, numerous long, oval ulcers or long, pointed, serrated scars are frequently found. In addition to the ulcers, a catarrhal inflammation of the mucous membrane is very apt to be present. 
Diffuse glanders manifests itself as a diffuse catarrh of the mucous membrane of the nasal and neighboring cavities with superficial ulceration, thrombosis of the veins, inflammatory infiltration of the submucosa, considerable thickening of the mucous membrane and the formation of a peculiar, radiating cicatrix.

The nodular and infiltrated forms are found in the lungs.

In the nodular form the lungs contain nodules* varying in size from a millet seed to that of a pea. They are gray by transmitted light, glassy and pearl gray by reflected light, and are surrounded by a hemorrhagic ring. The center of the nodules shows a pale yellow point in consequence of caseation and disintegration of the innermost cells. These nodules are of different sizes, of varying numbers, and of different ages. The formation of a capsule by a connective tissue membrane is induced by a reactive inflammation in the tissue surrounding the nodule. The nodules may be of an embolic origin, situated principally in the periphery of the lung, their structure being the same as that of the nodules on the nasal mucosa. Sometimes the lung nodules represent lobular pneunionic foci, in which the alveoli are filled with red and white blood corpuscles and with desquamated epithelium of the lungs. Central disintegration occurs very early. These areas are surrounded by a membrane resulting from a reactive inflammation which manifests itself and out of which a connective tissue capsule develops later on. In other cases, the foci suppurate forming cavities. Besides these nodules, there are often chronic bronchitis, peribronchitis, parabronchitis, atelectasis, inflammation of the tissue of the lung and less frequently circumscribed or exudative pleuritis.

Infiltrated glanders of the lungs form tumors from the size of a walnut to that of a child's head, consisting of a diffuse

*Nocard points out that when glandered horses are treated with mallein, a certain proportion of them recover in which case nodules that were present in the lungs cease to contain living bacteria, a fact he has fully proved by inoculation. On post-mortem examination the nodules may be readily felt by passing the hand with firm pressure over the surface of the lung which, when badly diseased, wiil feel like a bag full of shot or peas. 
glanderous infiltration of the alveoli and of the interstitial connectire tissue. Frequently on section the infiltrated parts of the lungs resemble very closely a soft sarcoma. They are of a dirty white color, of a gelatinous, juicy consistence, and irregular in shape. They may either become indurated so as to form hard, connective tissue like new growths (fibroma-like tumors of glanders, according to Gerlach), or they may become gangrenous. In nodular and in infiltrated glanders of the lungs, the bronchial glands, and frequently the mediastinal glands become enlarged, inclurated and studded with small foci of cell infiltration.

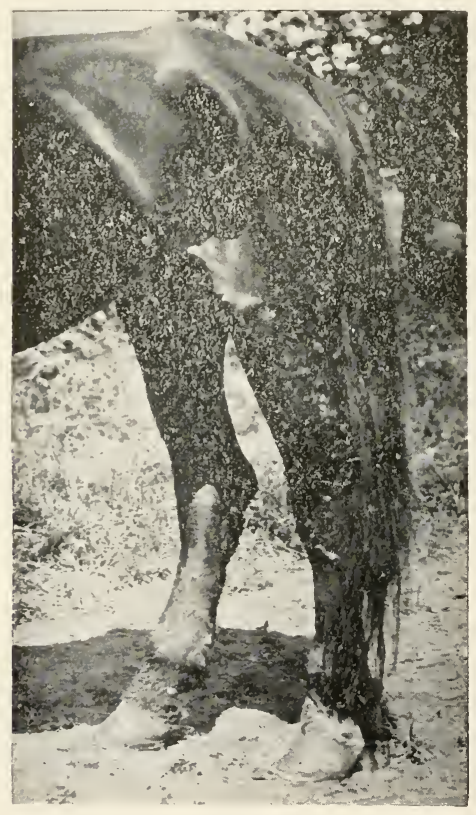

Fig. 22. Photograph showing farcy buds (cutaneous glanders).
In glanders of the skin (farcy) the nodules are found in the papillary layer, in the cutis and in the subcutaneous and superficial intermuscular tissue. The cutaneous nodules vary in size from a hemp seed up to that of a pea. They suppurate rapid$1 y$ and form small ulcers. The nodules in the subcutis are inflammatory (metastatic) tumors from the size of a pea to that of a hen's egg. They change into large abscesses and discharge externally. In the region of the nodules the lymphatic ressels are inflamed, swollen, and frequently resemble a rosary or knotted 
cord. Ulcers often develop from these secondary nodes. The neighboring lymph glands are at first swollen and soft, but later they become indurated by the growth of connective tissue and studded with dirty white nodules about as large as a pin's head, or with yellow foci of caseation. The capsule around the lymph glands becomes infiltrated with small cells and subsequently thickened. In rare cases secondary chronic farcy occurs. It is marked by a large diffuse new-growth of connective tissue with nodular thickening of the skin. This condition is termed glanderous elephantiasis or pachyderma. It chiefly affects the limbs and head.

Of the abdominal organs, the spleen is most frequently attacked. It then contains embolic nodules, varying in size and which either suppurate, or become calcareous. Similar nodules occur, though not so often, in the liver, kidneys, testicles, brain, muscles, heart, and bones. In the bones, a cellular infiltration of the medulla and purulent breaking down of the osseous tissue. Ulcers are very rare on the mucous membranes of the eyes, stomach and vagina. The blood shows signs of slight leucocytosis. The specific bacteria are found in the blood only in cases of acute general infection.

The anatomical changes in acute glanders consist chiefly in a disintegration of the respiratory mucous membrane, in a serous infiltration of the submucous, subcutis, and intermuscular tissue, with inflammation and suppuration of the lymph vessels and lymph glands. There are also metastatic formations in the skin and lungs. The nasal mucous membranes are covered with rapidly spreading ulcers with considerable infiltration into the submucosa. The mucous nembrane of the larynx and pharynx is swollen and covered with ulcers. The lungs are studded with purulent metastatic foci or fresh nodules. The skin is excessively swollen and covered with glanderous nodes. Sometimes diffuse gangrene of the skin occurs.

\$ ro3. Glanders in man. The symptoms of glanders in man are of much importance to the veterinary surgeon. Although the susceptibility to the disease is usually not very great, cases of human glanders unfortunately occur, especially 
among veternary surgeons and those having the care of horses. Human glanders is reported to be quite common in Russia. The parts usually first affected are the hands, nasal mucous membrane, lips and conjunctiva. After a period of incubation of from three to five days the infected part becomes swollen and painful, with subsequent inflammation of the lymph vessels and swelling of the glands. Fever is often the first symptom, and it is nearly always followed by a nasal discharge; ulcers on the nasal mucous membrane, pustules and abscesses in the skin, ulcers in the oral cavity, larynx, and conjunctiva, articular swellings, and grave general disturbances. Sometimes there is intense gastro-intestinal trouble. Nodules occur in the lungs in some cases. As a rule, death takes place in from two to four weeks, and occasionally in a few days. In other instances, the disease becomes chronic, with a duration of months ur years. Bact. mallei have been found in the blood in cases of acute glanders. The positive diagnosis depends on the possibility of infection having taken place, on inoculation in guinea-pigs, or the proof of the presence of Bact. mallei. Treatment is usually of no avail. The only hopeful cases are those that are purely local in their manifestation. A few of these are reported to have been cured by applying deep cauterization.

104. Differential diagnosis. Glanders is to be differentiated from a variety of nasal and lymphatic disorders more or less common in the horse kind. Before the discovery of the specific bacterium of glanders or of mallein, it was necessary to determine as closely as possible the differential anatomical characters between glanders and those of other affections, such as chronic nasal catarrh, strangles, lymphangitis, follicular ulceration of the nasal mucosa, cancer, sarcoma, actinomycosis, melanosis and the like. Since the discovery of practically a positive means of diagnosis, it does not seem wise to speculate upon the chances of the correct differential diagnosis by former methods. If the diagnosis can not be made from the manifestations of the disease, two reliable courses are possible although in most cases, but one (mallein) is to be recommended. These are (I) animal inoculation, and (2) the use of mallein. 
(1). Animal inoculation. Male guinea-pigs should be used. The material for inoculation usually consists of the nasal discharge from the suspected glandered horse, bits of scrapings from the ulcers, or pieces of other diseased tissue. The method to be followed is precisely the same as with the subcutaneous inoculation of tuberculous material. In these cases there is liable to be a local swelling and abscess. The first symptom of glanders noticed is usually orchitis. The lymphatic glands in the groin are also enlarged. After the orchitis becomes well advanced, the guinea-pig may be chloroformed and examined. Pure cultures of the specific organism can be obtained in most cases from the suppurating foci in the testicle. The spleen is usually enlarged and sprinkled with grayish nodules. Other organs may be involved.

(2) Mallein. Mallein is prepared in the same way as tuberculin. It consists of the glycerinated bouillon in which the glanders bacteria have grown and in which are the products resulting from their multiplication. It has a somewhat foetid odor. In applying the mallein test the horse is injected usually in the neck with the required amount ( 0.5 to 2 cc.) of mallein, the quantity depending upon the extent of concentration. If a concentrated mallein is used it should be diluted with a I per cent carbolic acid solution to at least $2 \mathrm{cc}$. The reaction is as follows. In a few hours there forms at the place of injection a hot, inflammatory swelling. It is very painful and in case of glanders quite large. From all sides of the swelling there may radiate wavy lines consisting of swollen lymphatics, hot and painful when touched, extending toward the adjoining glands. When the mallein injection is made aseptically, this swelling never suppurates, but it increases in size during a period of from 24 to 36 hours and persists for several days when it gradually diminishes and finally disappears at the end of eight or ten days. With the appearance of the local swelling the patient becomes dull and dejected, the eyes have an anxious expression, the coat is lusterless, the flanks contracted, the respiration hurried, the appetite is impaired, frequent shudders are observed to pass through the muscles of the fore legs and sometimes the trunk is subject to violent convulsive 
morements. The most active and fractions horses become listless and indifferent to their surroundings. These general phenomena constitute what the French call the organic reaction, but they are not always so clearly marked. Differences in their intensity are observed but they are never completely absent.

The temperature reaction never fails to show itself. In about eight hours after the injection the temperature of a glandered horse gradually rises $1.5^{\circ}, 2^{\circ}$ or $2.5^{\circ} \mathrm{F}$, and even more above the normal. The rise in temperature usually attains its maximum between the tenth and twelfth hour, occasionally not till the fifteenth, and more rarely not until about the eighteenth hour. An important fact to note is that the reaction called forth in glandered horses by the injection of mallein persists for from 24 to 48 hours and in some cases the temperature remains above the normal for even a longer time. In practice it is adrisable to take the temperature of the suspected animals two or three times before the injection of the mallein, and every two hours beginning at the eighth and going to the twentieth hour after the injection. It is often sufficient for diagnostic purposes to take the temperature but four times, viz., at 9, I2, I 5 , and is hours after the injection.

In healthy horses the injection of mallein, even in a much larger dose, produces no effect on the temperature or the general condition of the animal. There is produced, however, at the point of injection, a small oedematous swelling, somewhat hot and painful to the touch, but the oedema instead of increasing, diminishes rapidly and disappears in less than 24 hours.

The reaction called forth by the injection of mallein, in a glandered animal is quite specific. When it occurs one is enabled to state at once and with certainty that glanders exist, although the lesions may be quite minute or obscure. When the reaction does not take place it is generally considered that the aninial tested is not glandered, although the physical examination may suggest it. Notwithstanding the specific action of mallein, its administration can give really useful indications according to Nocard "only when, and as far as, we 
can remove the causes of error that have been pointed out by experience." For example it would be imprudent to use mallein in case of animals already suffering with an abnormal temperature. The further precautions should be taken that the animals subjected to the test are remored as far as possible from atmospheric variations and the influence of strong sun light, fog, rain and air currents. If it be true that majority of horses are not susceptible or nearly so, to these influences, there are still some that are affected by them. So that a rise of 1.5 or 2 degrees in temperature would not necessarily indicate a reaction. Again, it must not be forgotten that certain diseases, strangles for instance, produce frequently great daily rariations in the temperature, therefore, when there is reason to believe in the presence of a clisease of this kind, it is necessary to make sure that the increase of temperature consequent on injection of mallein is persistent, and that the organic reaction is clearly present.

The question arises whether animals found by the help of mallein to be glandered ought to be immediately slaughtered? Nocard says no. The experience of the last few years goes to prove that among the animals that react there are some which, when removed from the infected center and thereby withdrawn from all chance of new contamination, recover. "We ought therefore,"' he continues, "to confine ourselves to the destruction of those which in addition to the reaction, present some clinical indication of the disease, such as ulceration of the nose, indurated glands, suppurative lympliangitis, sarcocele or other pronounced manifestation of the disease. The animals not showing physical signs of affection must simply be removed from among the healthy horses and subjected from time to time, say erery two months, to the mallein test. If any of these should eventually show the clinical signs of glanders they ought to be slaughtered at once. On the other hand, those animals which have stood two successive doses of mallein without reacting ought to be considered definitely cured, restored to their places and put to the free disposal of the owners.'

The riews of Nocard are not universally entertained in this country. It has been shown repeatedly that a good reaction, fol- 
lowing the injection of mallein, was a sure indication of glanders as revealed by post-mortem. The question, however, concerning the necessity of immediate slaughter for purposes of protection, when there are no evidences of lesions on physical examination, seems to be an open one. This question which pertains to sanitary police rests, until the results of conclusive investigations are recorded, with those entrusted with the protection of animals and men from this disease. Howerer, the results of certain experiments in the use of mallein as a therapeutic agent, and the fact that certain animals recover when kept in quarantine are very suggestive. Certainly further investigations are needed to determine the safe and equitable disposition of animals devoid of all symptoms and obvious lesions of glanders, but which give a reaction to the mallein test.

\section{REFERENCES.}

I. BABEs. Observations sur la morve. Arch. de Med. Exper. et d'Anat. Pathologie. T. III. (1S9I) p. 6 Ig.

2. Butren. Glanders. Bulletin No. 16. Miss. Agr. Expt. Station. $189 I$.

3. CARy. Glanders. Bulletin No. 35. Ala. Agr. Expt. Station of the Agricultural and Mechanical College. Jan. ISg2.

4. DE SCHWEINITZ AND KILBORNE. The use of mallein for the diagnosis of glanders in horses and experiments with an albumose extracted from cultures of bacillus mallein. Am. I'et. Review. Vol. XVI. (IS92.) p. 439.

5. Frixcis. Glanders, tests witl mallein. Bulletin No. 30 . Texas Agri. Exp. Station. IS94.

6. FROTHINGHAII. The diagnosis of glanders by the Strauss method. Jour. of Medical Research. Vol. VI. (I901). p. 331.

7. LOEFFLER AND SCHÜTz. On the bacillus of glanders. Deutsch Med. Wochenschrift. Dec. IS82. Translated, Vol. CXV. New Sydenham Society.

S. M'FADVEAN. The pulnionary lesions of glanders. The four. of Comp. Path. and Therapeutics. Vol. IX. (1S96). p. 50 .

9. NocARD. The value of mallein as a means of diagnosis in doubtful cases of glanders. The Jour. of Comp. Path. and Therapeutics. Vol. VIII. (IS95). p. 227.

10. RevNoLDs. State control of glanders in Minnesota. The Jour. of Comparative Medicine and Veterinary Archives. Vol. XX. No. 12, December, 1899 ,

11. Schütz. A contribution to the subject of glanders. The Jour. of Comparative Pathology and Therapeutics. Vol. XI. (ISgS). p. I.

12. SuITH. On the influence of slight modifications of culture media on the growth of bacteria as illustrated by the glanders bacillus. Joumal of Comparative Medicine. (iSgo). p. I5s.

13. STRAUSS. Sur un moyen diagnostique rapide de la morve. Arch. de. Med. Exper. et d' Anat. Path. T. III. (Iss9). p. 460.

it. Wrildius. Glanders. Bulletin No. t. Montana Agric. Expt. Station. IS94. 
TUBERCULOSIS.

Synonyms. Consumption; pearl disease ; phthisis ; scrofula (pigs).

$\$$ ro5. Characterization. Tuberculosis is an infectious disease from which the human species, cattle and swine suffer very extensively and which, under favorable conditions, attacks nearly if not all species of animals including fish. It is a disease of slow development, involving either primarily, or in association with other organs, the lymphatic system. It is characterized by the formation of nodules, or tubercles, in consequence of the activities of the specific Bacterium tuberculosis. It does not destroy life by acute toxæmia, but by a chronic and long continued systemic poisoning and by the morbid changes brought about through the localization of these lesions in organs necessary to life.

$\$$ ro6. History. Tuberculosis is one of the oldest diseases affecting cattle of which there are identifying records. It seems to have been known to the Jewish people during their Egyptian captivity and the ecclesiastical laws for many centuries contained numerous enactments against the consumption of flesh from tuberculous animals. In 1370 , it was forbidden in Munich to have on sale the flesh of animals affected with tuberculosis. A number of other cities passed similar ordinances. In 1702 , Florinus described the disease and emphasized the then existing opinion that it was identical with syphilis. This led to the practice of destroying all tuberculous animals. In 1783 , the Berlin Board of Health rejected the theory of the connection of tuberculosis and syphilis and declared the flesh of affected animals to be fit for food. This led finally to the cancelling of all laws throughout Prussia against the use of flesh for food from animals affected with the disease. Tscheulin, in 1816 , recognized in reference to the infection of meat, three degrees of bovine tuberculosis, viz: (1) in which the tubercles were to be removed; (2) in which the diseased parts were to be destroyed and the meat sold at a low price; and (3) those cases in which the lesions were so extensive that the whole carcass must be rejected. 
The study of the lesions themselves gave rise to a number of beliefs concerning their nature. Thus Virchow, Scliuppel and others declared that the tubercles in cattle were lymphosarcomata. Leisering considered them simply as sarcomata. Spinola and Haubner maintained that human and bovine tuberculosis were identical.

In 1865 , Villemin showed that tuberculosis was due to a specific infection. He produced the disease in rabbits by inoculating them with tuberculous material from human subjects. He also produced the disease by feeding experimental animals and by causing them to inhale tuberculous material. Chauveau, in the same year, produced the disease in cows. These results were soon confirmed by Klebs, Colnheim and Gerlach. These experiments in which the disease was produced in one species with tuberculous material from another followed by the discovery by Koch of the specific bacterium of the disease, led to the view that tuberculosis in all species of mammals was identical. This generally accepted belief caused sanitarians to look upon tuberculosis in cattle as a great menace to public health with the result that during the closing decade of the last century, this disease in cattle was treated more vigorously as a menace to the liuman species than as a destructive disease of animals.

In 1896 , Dr. Theobald Smith pointed out the fact that for certain animals the tubercle bacteria from cattle were more virulent than those from man and further, that there were certain morphological and cultural differences existing between them. In 1898 , he published the results of a more extended series of investigations. Since that time a number of investigators have arrived at the same conclusion. The fact has come to be well known that certain differences exist between the bacteria of tuberculosis found in the human and in the bovine species. Koch's experiments reported at the tuberculosis congress in London in July 190I, give additional evidence of a difference in virulence for experimental animals of the bacteria of human and of bovine tuberculosis. To what extent the human species becomes infected from the bovine kind cannot be stated, but the accumnlating evidence tends to 
the conclusion that borine tuberculosis is of less significance in its influence upon public health than has heretofore been thought, and of more importance as a rapidly spreading and destructive disease among cattle. It is not proven, however, that the human species is not affected with the bacteria of bovine tuberculosis. The circumstantial and direct evidence is very conclusive that it is, but that such infections are not so numerous as previously supposed. Concerning its transmission, the conclusion seems to be warranted, that the virus of tuberculosis spreads very largely among men and cattle from individual to indiridual of the same species rather than from species to species.

$\$$ 107. Extent of tuberculosis, especially among cattle and swine. The committee on cattle diseases and animal food of the American Public Health Association for Igor reported the appended statistics concerning the extent and increase of tuberculosis in cattle and swine in various countries.

"The slaughter house statistics of Prussia show I 4.6 per cent of the cattle and 2. I 4 per cent of the swine to be tuberculous. In Saxony the percentage is 29.13 with cattle and 3. Io with swine. In the city of Leipzig the figures are 36.4 for cattle and 2.17 for swine. (Siedamgrotzky). Of 20,850 animals in Belgium tested with tuberculin in $1896,48.88$ per cent reacted. Of 25,439 tested in Denmark from I 893 to I 895 , 49.3 per cent reacted; and of 67,263 tested from 1896 to I 898 , 32.8 per cent reacted ( $\mathrm{Bang}$ ). An examination of 20,930 cattle in Great Britain, either slaughtered and examined postmortem or tested with tuberculin, showed 5,44I or 26 per cent affected with tuberculosis. M'Fadyean estimates that 30 per cent of the cows in Great Britain are tuberculous. Figures available in the United States do not cover a sufficient area of our territory to allow us to make a reliable estinuate of the extent of tuberculosis in milch cows."

"Our beef cattle as they come to the large packing houses, are yet comparatively free from tuberculosis. Of 4,841 , 166 cattle slaughtered in the year I 900 , under Federal meat inspection, but 5,279 or O. I I per cent were sufficiently affected to cause the condemnation of any part of the carcass. Of $23,336,884$ 
hogs sinilarly inspected, $5,+40$ were sufficiently affected to cause a condemnation of some part of the carcass. This is equal to 0.023 per cent, or slightly more than one-fifth the proportion found in beef cattle."

"The slaughter house statistics of all countries show that the percentage of affected hogs increases as the disease becomes more common in cattle, so that we must consider not only the effect of the disease upon beef and nilk producing animals, but also upon swine. Tuberculosis is more acute with hogs than with cattle, and there is a much greater tendency to generalization, consequently the parts used for human food are more likely to be affected, and if there is a possibility to communicate the virus through the meat the danger is increased by this peculiarity of the disease in swine."

$\$$ 108. Geographical distribution. Tuberculosis is an exceedingly wide spread disease. In earlier times it was quite prevalent among cattle in central Europe. It seenis to have existed in Western Asia and Northern Africa at an early date. From these centers it has spread to nearly every cattle raising country of the world. Its rapid spread during the last fifty years is attributed to the increase in cattle exchange resulting in the introduction of tuberculous animals into healthy herds. It is stated that in many countries, and in large districts within others, tuberculosis did not exist until it was introduced within recent years by the importation of diseased animals.

In countries where there has been little or no importation of cattle, and in which the native breeds still exist unchanged, as in many parts of Russia, Austria and Spain, in the northern part of Sweden and Norway, and in parts of Africa, tuberculosis is practically unknown. This is true of the cattle on the island of Jersey where for more than a hundred years foreign cattle have not been introduced.

In the United States the disease is very widely distributed. It is found to a considerable extent in certain localities where the climatic conditions seem to be beneficial for tuberculous people. The explanation for this seems to be that tuberculous animals have been introduced into certain herds in these districts. There are, however, large areas in which it is practi- 
cally unknown. The Western steers that are killed in the slaughter houses of Kansas City, Omaha, Sioux City and Chicago are practically free from this disease. In many localities, especially where there is an extensive interchange of animals, a large percentage of the herds are more or less affected.

Iog. Etiology. Tuberculosis is caused by a rodshaped organism known as Bacterium tuberulosis. It was discovered by Robert Koch in I882. Schiiller and Toussaint had previously studied growths which seem, from the results of their inoculation experiments, to have been this organism. The bacterinm of tuberculosis is a slender rod-shaped organism with rounded ends, from 2 to $5 \mu$ in length and from o 3 to $0.5 \mu$ broad. The rods

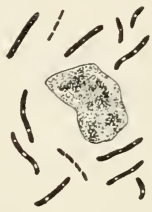

FIG 23. Bacterium of tuberculosis. are straight or slightly curved, and occur singly, in pairs or in small bundles. Frequently they cross one another. They do not produce spores, but vacuoles are often observed and branching forms have been described.

The bacterium of tuberculosis is readily cultivated on artificial media such as blood serum, glycerinated agar and bouillon, and potato after it has been adapted to such artificial conditions.* It is, however, not easy to cultivate it directly from

* To accomplish this necessitates a very special and careful procedure. Dr. Theobald Smith, of Harvard Unixersity (Jour. of Exp. Med., Vol. III., IS98, p. 45r), has the credit of formulating a method by combining details in such a manner that the procuring of cultures is, in most cases, possible. Dog serum is used. The method, as he gives it, is as follows, viz. :-

"The dog was bled under chloroform and the blood drawn from a femoral artery, under aseptic conditions, through sterile tubes directly into sterile flasks. The serum was drawn from the clots with sterile pipettes and either distributed at once into tubes or else stored with 0.25 to o 3 per cent chloroform added. Discontinued sterilization was rendered unnecessary. The temperature required to produce a sufficiently firm and yet not too hard and dry serum is for the dog $75^{\circ}$ to $76^{\circ} \mathrm{C}$. For horse serum it is from $4^{\circ}$ to $5^{\circ}$ lower. The serum was set in a thermostat into which a large dish of water was always placed to forestall any abstraction of moisture from the serum. About 3 hours suffice for the 
ordinary tuberculous lesions. Although in the beginning, the tubercle bacteria from man and from animals were believed to be entirely identical, they have been found to possess slightly different characters and properties. Smith pointed out in I 898, that morphologically those from cattle were shorter and thicker than those from man, that they grew slightly different on blood serum, and that they were much more virulent for cattle and rabbits than those from the human species. Since that time his conclusions have been confirmed by a number of investigators. Koch has recently obtained like results. At present, therefore, we must look upon the tubercle bacteria coming from these different species as possessing races or varieties which, perhaps, are the results of their different conditions of life. The investigations which have been made with the decidedly different forms of this organism found in in tuberculosis of fowls and of fish have led a few experimenters to believe that they are simply varieties of the organism first described by Koch. Further inquiries are necessary to fully satisfy bacteriologists that all of these forms are thus related to the one species. There seems to be no reason for doubting that the bovine and human forms are varieties or races of the same species. The difference in the conditions of life under which they exist in the bodies of men and of cattle are quite enough to explain resulting differences in the bacteria.

coagulation. When serum containing chloroform is to be coagulated, I am in the habit of placing the tubes for an hour or longer in a water bath at $55^{\circ}$ to $60^{\circ} \mathrm{C}$, , or under the receiver of an air pump, to drive off the antiseptic. This procedure dispenses with all sterilization excepting that going on during the coagulation of the serum. It prevents the gradual formation of membranes of salts, which, remaining on the surface during coagulation, form a film unsuited for bacteria. Tubes of coagulated serum should be kept in a cold closed space where the opportunities for evaporation are slight. They should always be kept inclined.

"The ordinary cotton-plugged test tubes I do not use, because of the rapid drying out permitted by them, as well as the opportunities for infection with fungi. Instead, a tube is used which has a ground glass cap fitted over it. This cap contracts into a narrow tube plugged with glass wool. This plug is not disturbed. The tube is cleaned, filled, and 
I Io. Symptoms. The symptoms vary according to the course of the disease. Two classes are recognized, viz.: (I) the chronic form, which is most common, and (2) the acute form or miliary tuberculosis.

The symptoms of chronic tuberculosis depend upon the location of the lesions and their extent. When the lesions are situated deeply and are not of great extent, they may exhibit no visible evidence of their presence. In such cases the infected animal may present the picture of perfect health and show no disturbance of function. Indeed, some animals in which

inoculated by removing the cap. With sufficient opportunity for the interchange of air little evaporation takes place, and contamination of the culture is of very rare occurrence. In inoculating these tubes, bits of tissue, which include tuberculous foci, especially the most recent, are torn from the organs and transferred to the serum. Very little crushing, if any, is desirable or necessary. I think many failures are due to the often futile attempts to break up firm tubercules. Nor should the bits of tissue be rubbed into the surface, as is sometimes reconmmended. After a stay of several weeks in the thermostat, I usually remove the tubes and stir about the bits of tissue. This frequently is the occasion for a prompt appearance of growth within a week, as it seems to put certain still microscopic colonies in or around the tissue into better condition for further development. The thermostat should be fairly constant, as urged by Koch in his classic monograph, but I louk upon moisture as more important. If possible, a thermostat should be used which is opened only occasionally Into this a large dish of water is placed, which keeps the space saturated. Ventilation should be restricted to a minimum. As a consequence, moulds grow luxuriantly and even the gummed labels must be replaced by pieces of stiff manila paper fastened to the tube with a rubber band. By keeping the tubes inclined, no undue amount of condensation water can collect in the bottom, and the upper portion of the serum remains moist. The only precaution to be applied to prevent infection with moulds is to thoroughly flame the joint between tube and cap as well as the plugged end, before opening the tube. When test tubes are employed it is well to dip the lower end of the plug into sterile molten paraffin and to cover the tube with a sterilized paper cap. The white bottle caps of the druggist are very serviceable."

While the tuberculous material is perfectly fresh (uncontaminated) and in the early stages of the disease, it is safer to inoculate a gininea pig, and after the lesions begin to develop to chloroform it and make the cultures from the recently affected liver or spleen. 
the lesions are both extensive and widely distributed and which have never presented noticeable signs of the disease, are killed in prime condition for beef without a suspicion of the presence of disease.

Since the lesions of tuberculosis vary so much in different cases, it is not possible to give a description of what can be designated the characteristic or eren the usual symptoms of this disease. There are, however, certain general manifestions that appear in most of the advanced cases, such as emaciation, while the appetite continues good. This is always a suspicious indication and especially if accompanied by cough, rough coat and tight, harsh skin. Rough or loud respiratory sounds are suspicious, and, in advanced cases, it is often found that the animal groans when pressure is brought to bear upon the chest wall. Many cases bloat habitually, and hard, painless swellings (enlarged lymphatic glands) beneath the skin in the region of the escutcheon, flank, shoulder or throat are suspicious.

In tuberculosis of the lungs, it may be said that coughing is the most noticeable symptom. It is most common after feeding, drinking, or after rapid moving following a period of repose, but sometimes it occurs without any apparent cause. The cough is usually strong, dry and frequently of a high pitch. Sometimes it is very violent accompanied by protrusion of the tongue. Auscultation reveals modified and abnormal sounds of different kinds in the lungs ; sibilant, sonorous and mucous rales are most common. A dull sound is often detected on percussion. It is also to be noted that this condition is of slow development and long duration, thus aiding one to distinguish it, in many cases, from bronchitis or pneumonia.

Where the mediastinal lymphatic glands are enlarged and press upon the oesophagus, it is stated that the animal bloats habitually. Chronic or habitual bloating accompanied by a good appetite and no other evidence of disease of the digestive tract, especially if there is shortness of breath and cough, may be looked upon as strongly indicative of tuberculosis with enlarged mediastinal lymphatic glands.

Sometimes large tubercular masses develop on the pleura. In such cases the principal symptom is a friction sound that is 
heard most distinctly during inspiration. If the nusses are large enough they give rise to a dull sound upon percussion. In tuberculosis of the stomach and intestines, digestion is interfered with. This gives rise to poor appetite, frequently to diarrhoea and sometimes to alternation of diarrhoea and constipation. In tuberculosis of the peritoneum or of the lining of the abdominal cavity, the lymphatic glands of the flank are often enlarged and hard. Sometimes this condition can be diagnosed positively by a rectal examination and the discovery by this means of the hard, nodular masses. Tuberculosis of the liver does not give rise to obvious symptoms unless the disease is far advanced, in which case jaundice may be observed.

In animals in which the post-pharyngeal lymphatic glands are enlarged from tuberculosis, the breathing is harsh and noisy. In this condition there is sometimes difficulty in swallowing, and particles of chewed up food are occasionally expelled from the mouth, either voluntarily, when it is found that they cannot be swallowed conveniently, or by the cougling they occasion upon reaching the pharynx. These enlarged glands may sometimes be detected by palpation accomplished by placing one hand on each side of the throat above the larynx and then pressing from opposite sides.

Tuberculosis of the udder is detected by an enlargenent and liardening of the affected part, usually by the absence of pain and the fact that the secretion is not altered until the part has been diseased for some time. In advanced cases, instead of milk, the udder secretes a yellowish, cloudy and sometimes flocculent fluid. In acute, rapidly developing cases, there may be pain and oedema of the skin. In nearly all cases of udder tuberculosis the supra-mammary lymphatic glands, situated above the udder in the middle of the escutcheon are enlarged and hard. If there is doubt as to the character of the disease of the udder, the milk, or possibly a piece of excised udder tissue, may be examined bacteriologically.

In tuberculosis of the brain, the animal is unsteady and uncertain in its movements. It lies down much of the time, is usually subject to occasional cramps and is apt to carry the head in an unusual position. Such cases are inclined to in- 
crease rapidly and terminate in death following coma or convulsions. In tubercular disease of the bones and joints, the parts are enlarged, there is loss of motion, pain and usually abscess formation followed by the discharge of thick yeliow pus. In tuberculosis of the uterus or ovaries and sometimes in peritoneal tuberculosis in the cow, the subject is almost continually in heat. In tuberculosis of the uterus, there is sometimes a discharge of thick, yellowish material mixed with inucus and slime. In tuberculosis of the testicles the organs become enlarged and hard.

In all adranced cases the nutrition of the animal is interfered with and, sooner. or later, the "tuberculous cachexia" appears. It is, however, in many cases remarkable to note the extent of lesions in animals that are well nourished and present no external signs of disease. Aninals killed in prime condition by the butcher are sometimes found to contain extensive and widely distributed lesions of tuberculosis. In general tuberculosis, many of the symptoms described above may occur simultaneously. The symptoms of acute miliary tuberculosis " galloping consumption" are rapid loss of flesh, depression, poor appetite, congh, weakness, rapid breathing, harsh respiratory sounds, some elevation in temperature, increased pulse rate and, sometimes, enlarged lymphatic glands. The course of this form of tuberculosis is always rapid and terminates in death. Acute miliary tuberculosis occurs when large numbers of tubercle bacilli are discharged into the blood or lymph currents. They are then carried to other paits of the body, filtered out in the capillaries of the lungs, liver, spleen, kidneys and elsewhere, causing tubercular lesions in each of these localities. The lesion from which the infectious material entered the circulation may have been a comparatively snall nodule. This form of the disease is more likely to appear in young animals than in adults, and is more common among swine than in cattle. 
SIII. Morbid anatomy. The usual direct ana tom ica 1 changes following the invasion of tubercle bacteria are the formation of nodules or tubercles. A tubercle has been defined as, " a small nonvascular nodule composed of cells varying in form and size with some basement substa nce between the $m$ and with an inherent tendency to undergo central necrosis." In a large number of cases the individual tubercles are distinct and easily recogniizable while in others they are coalesced forming a mass of lesions vary, there- posits. (Smith.)

fore, from well isolated minute or larger nodules to masses or carities containing a purulent, caseous, or calcified substance.

The location of the primary lesion depends upon the channel of infection. If the specific organisms are lodged in the oral cavity or pharynx they may, through an accidental abrasion of the mucosa, be taken to some of the lymphatic glands about the head; if they are taken directly through the respiratory passages into the lungs they either develop nodules in the lung tissue proper, or they are carried through the lymphatic system to the lymph glands draining the lungs where the lesions first appear. If the specific bacteria are first lodged in the 


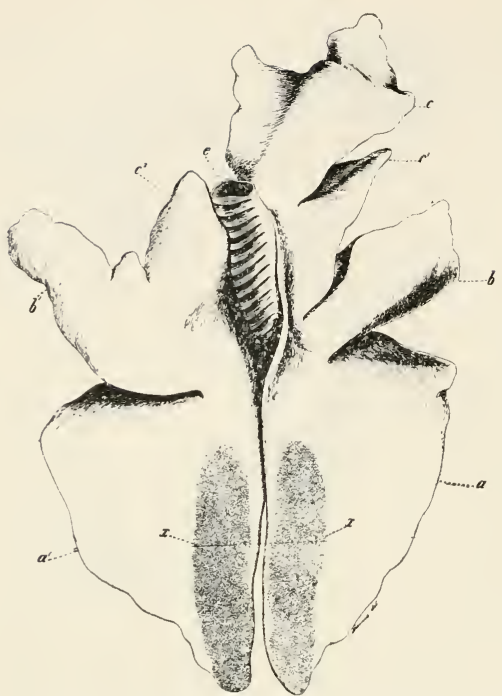

FIG. 25. Dorsal aspect of bovine lungs. a a $a^{\mathrm{I}}$ right and left caudal lobes. $b-b^{\mathrm{x}}, r$, and $l$. ven- however, seems to tral lobes. $c-c^{1}$, first and second right cephalic show that in a large lobes. c', left cephalic lobe. $c$, trachea. $x x$, majority of cases region most frequently involied in the earliest stages of pulmonary tuberculosis. The lesions are usually at this stage embedded in the lung are (1) in the lungs tissue. (Smith.)

or the lymphatic glands draining them, (2) in the lymphatic glands about the head, (3) the intestines and mesenteric glands, and (4) in the portal glands or liver substance itself. Primary lesions sometimes occur in the generative organs and udder.

It not infrequently happens that the apparent primary lesions occur on the pleura, peritoneum, meninges or synorial membranes while the organs remain free from disease. In such cases the lesions consist of many tubercles varying from one to ten or nore millimeters in diameter or of bunches of closely set tubercles which are more or less flattened or irregular 
in shape owing to their mutual pressure (Fig. 3I). Sometimes these tubercles are attached to the serous membrane by a small, tough, fibrous pedicle ; frequently, however, this is absent and the nodules rest bodily upon the membrane.

The structure of the tubercle consists, in the beginning, of a few cells surrounding the invading specific organisms. These are soon encased by a zone of epithelioid cells and giant cells which is soon surrounded by an outer layer of round or lymphoid cells. The central portion becomes necrosed and as the nodule enlarges the central necrotic portion becomes correspondingly large. In cattle there is a strong tendency for the necrotic tissue to become infiltrated with line salts

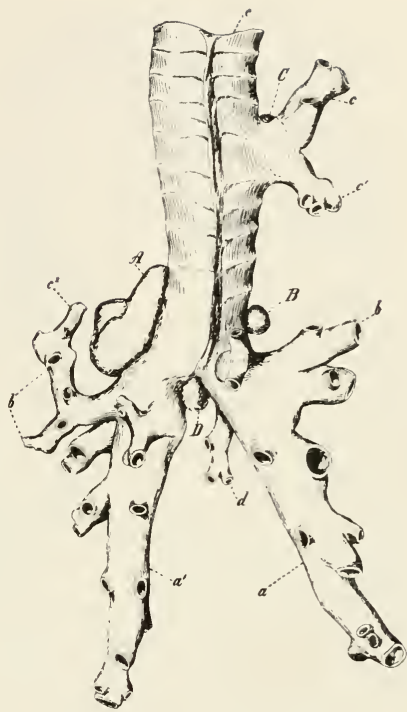

FIG. 26. Trachea and bronchial tubes of bovine lungs showing attached bronchial glands. a.a., supply right and left caudal lobes. b.b., supply $r$. and l. ventral lobes. $c-c^{\mathrm{I}}$, branches of its right supernumerary bronchus. $c^{2}$, supply left cephalic lobe. d, branch to azygos lobe. e, trachea. A, left bronchial lymph gland. $B$, right bronchial lymph gland. $C$, lymph gland base of supn. bronchus. $D$, gland often between bronchi. The glands A. to D.are often involved. (Smith.) and encapsulated (Fig. 32). In certain other species a deposit of fibrous tissue in the outer zone of the tubercle has been observed. In the smaller and more susceptible experimental animals such as the guinea pig and rabbit and frequently in swine, the lesions are of a more diffuse nature infiltrating the interstitial tissue with the tuberculous mass and gradually encroaching upon the parenchyma. Circumscribed tubercles may also be present. 
In secondary or generalized tuberculosis one or more of the organs, onentum, serous membranes, or lymphatic system may become more or less thickly sprinkled with minute greyish nodules about the size of a millet seed. These tubercles are at first almost the color of mother-of-pearl but later as the central caseous degeneration begins they become grayish. Giant cells are usually numerous.

In studying the lesions in a fatal case of tuberculosis one may find with varying modifications one or more of the following conditions, viz.

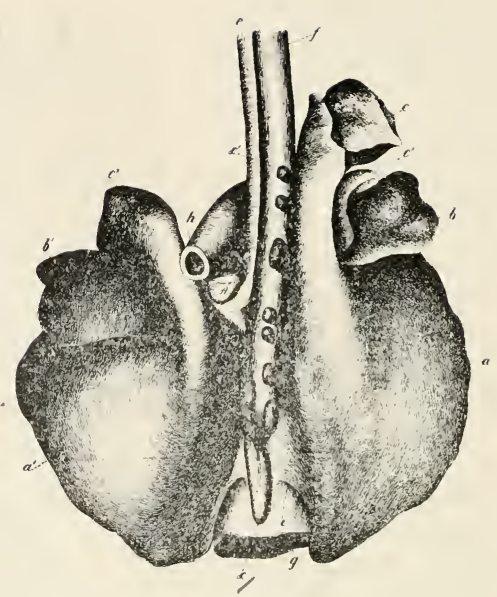

FIG. 27. Dorsal aspect of bovine lungs show. ing position of the postcrior mediastinal glands. $a, b, c, c^{\prime}$. caudal, ventral, cephalic lobes. $f$. oesophagus. g. muscular pillars of diaphragmh. posterior aorta. A. left bronchial gland. $i$. caudal margin of the ligament of the lung. Mediastinal glands are shown, most of them resting on the oesophagus. The large caudal gland resting on the pillars of the diaphragm is most frequently diseased and often attains an enormous size. The remaning mediastinal glands are arranged in two sets on the right and left margins of the oesophagus. (Smith.)

(1) The primary lesion may be found in any one of the organs or membranes. Its comparative age is determined by the character of the anatomical changes. It may be entirely encysted, caseous or calcareous and dead. In addition to this primary focus, there may be a succession of tubercles of various ages distributed in one or more organs.

(2) The lesions may be restricted to one organ, as the liver in which the primary focus has spread by continuity due 
Plate II.

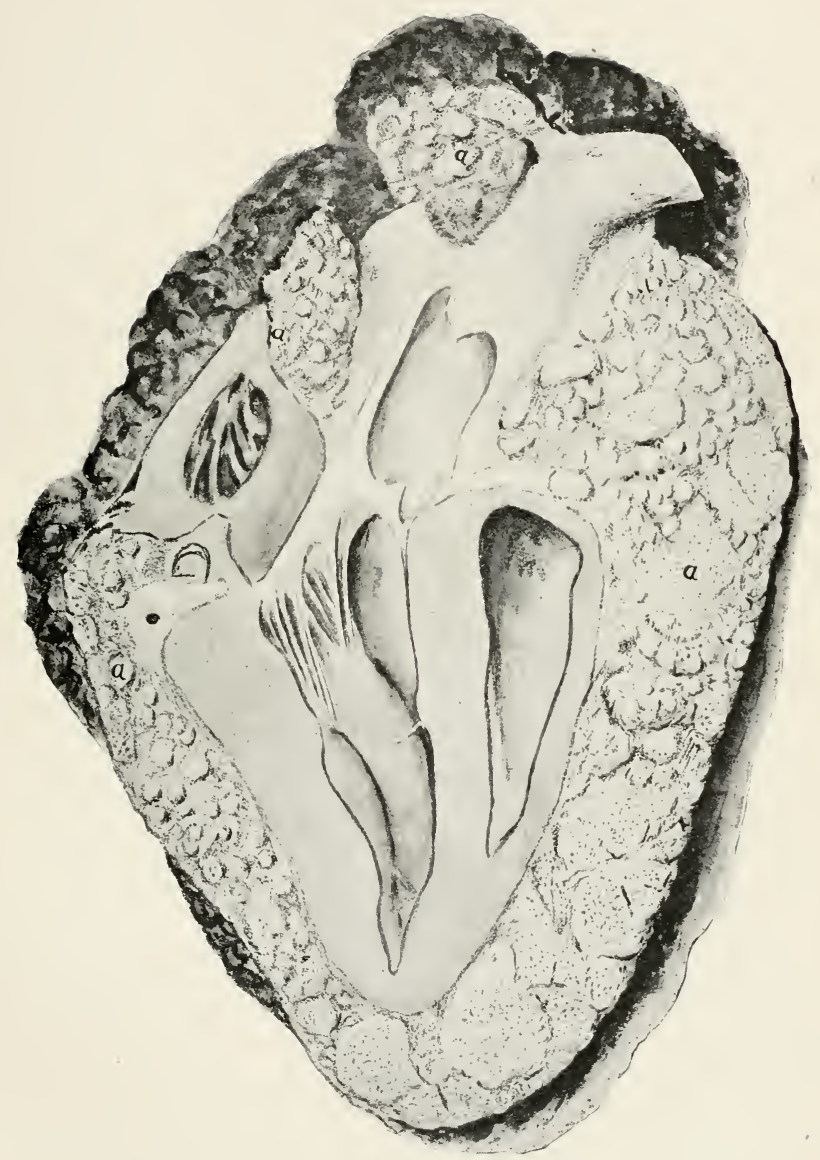

TUBERCULOUS HEART FROM A COW. 

to its infiltrating nature until the destruction of the tissues of the organ has become so extensive that death results. Such cases do not seem to be common.

(3) The primary lesion may be well marked and accompanied by miliary tubercles sprinkled extensively throughout the organs and tissues of the entire body.

(4) The lesions throughout the body may resemble each other very closely so that difficulty may be experienced in determining the primary focus.

In the lungs, two distinct forms of lesions are observed. (I) The air cells may be infiltrated with the tuberculous mass spreading directly from the primary focus. This may be purulent, caseous or calcareous. The color may be whitish, gray or of a yellowish tinge. (2) The lesions may consist of miliary tubercles. In later stages these nodules, more or less translucent, may become yellowish, caseated and calcareous in their centers. Large tubercular nodules are frequently formed by the massing of sereral of these minute tubercles.

When the lungs are primarily attacked the caudal (principal) lobes are most frequently involved. Smith considers the seeming predilection for the larger lobes to be due to

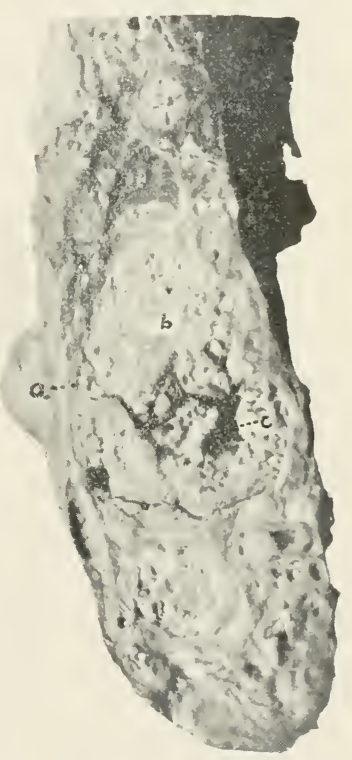

Fig. 2S. Tuberculous focus mechanical conditions. The with center. (c) undergoing supwriter has found, however, that puration. (a) caseous (b) calcarin certain herds that have been eous centers.

killed after the tuberculin test, the primary and only lung lesions were in the ventral and cephalic lobes. It is important 


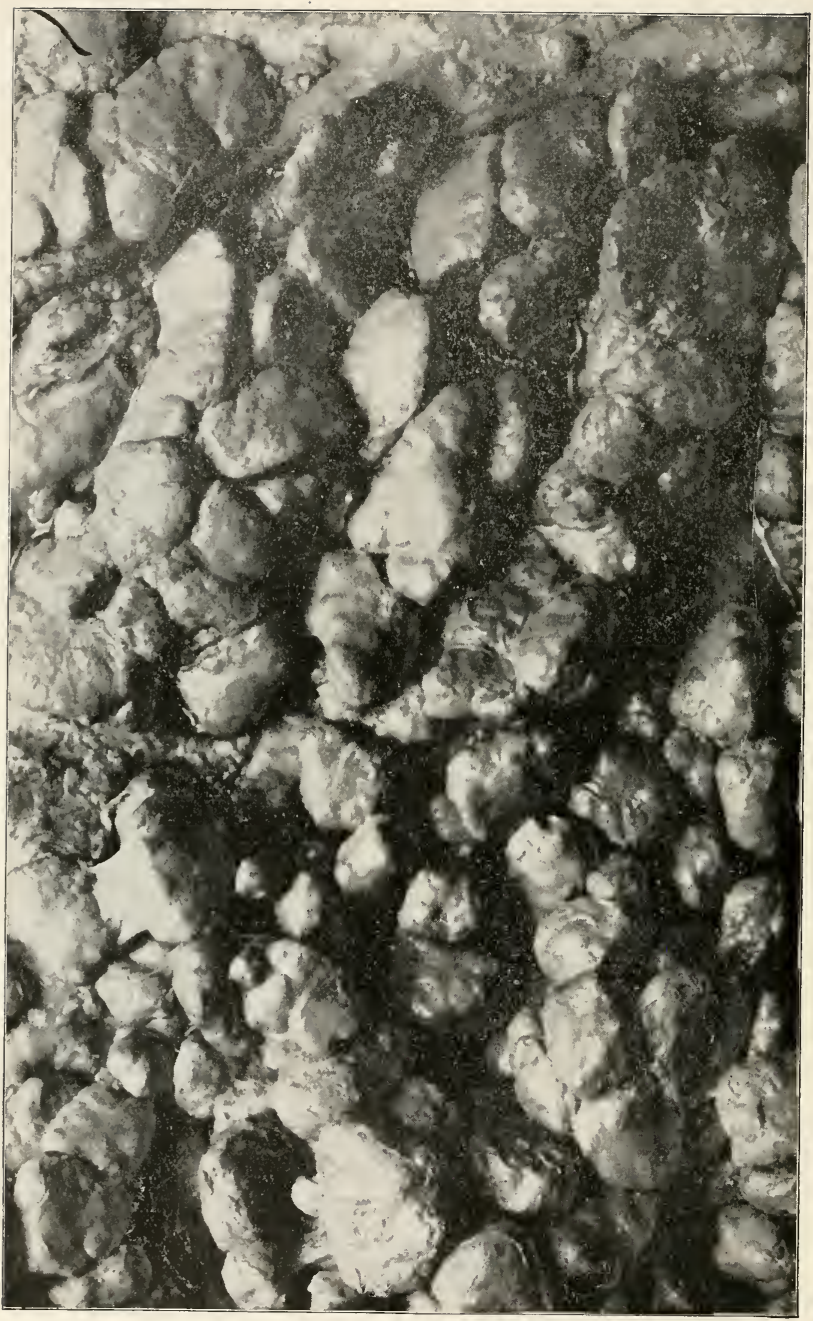

FIG. 29. Photograph of a portion of tubercular omentum of a cow, showing the tubercles, natural size. 
to note that usually the bronchial glands are also involved. When the pleurae are affected the lesions consist of nodules varying in size from that of a millet seed to a large pea, sprinkled more or less thickly on one or both of the visceral or parietal surfaces. These form the "pearl disfase" (Perlsucht) of the German and the "grape disease' of the English writers. If they become confluent, large masses are found.

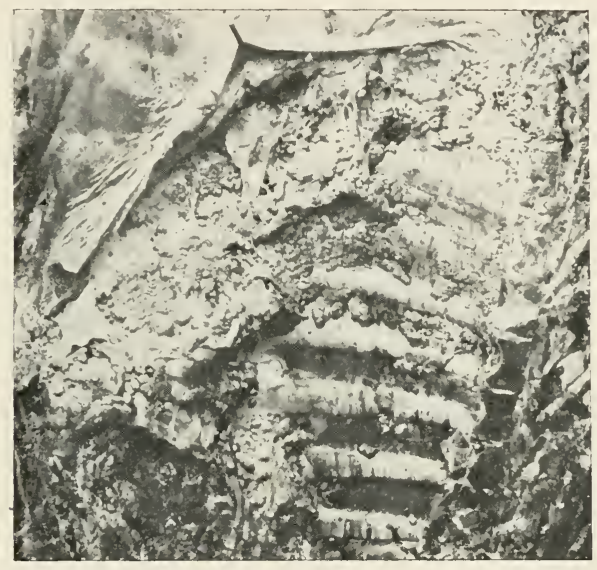

FIG. 3o. Photograph of pleura showing small tubercular nodules.

Tuberculosis of the thoracic glands is very common and usually accompanies lesions in the lungs; but often the lungs may be healthy and the glands involved. (See figures for location of glands). The primary lesions may be and often are found in the lymphatic glands about the head.

In the abdominal cavity the organs most frequently involved are the peritoneum, mesenteric lymph glands, portal lymph glands and liver. The kidney's, spleen, ovaries and uterus are more rarely the seat of tuberculous tumors. Ulcers in the intestine have not been common in the writer's observation. Tuberculosis of the testis is sometimes found. The udder 
often becomes the seat of tubercular deposits in cases of generalized tubercnlosis.

When the primary infection is restricted to a single focus the disease is said to be localized. When the specific bacteria are spread from the primary lesion through the agency of the lymph and blood streams, sprinkling other organs with the infecting bacteria each of which becomes the starting point for the development of a new tubercle, the disease has become generalized. $*$

It was formerly considered that when the lesions existed in both of the large (abdominal and thoracic) cavities of the body the disease was generalized. It is possible, however, for it to be generalized when the lesions are restricted to the organs of one cavity as the secondary seeding with the bacteria that have escaped from a primary focus through the circulation may be restricted to the cavity in which the first lesion developed. It seems better, therefore, to accept Ostertag's views and classify local and general tuberculosis in accordance with the nature of the lesions rather than their distribution in the body.

The fact is worthy of consideration, that very often cattle killed after reacting to tuberculin do not show extensive distribution of lesions. Frequently animals are killed soon after infection has taken place, in which case the lesions are restricted to a single lymphatic gland or other organ. In other cases old lesions of considerable proportion are found as shown in plate II, where the heart muscle was entirely encased in a thick calcareous tubercular deposit. In this case, the animal was in good flesh and killed for beef without a suspicion that it was in any way diseased.

SI12. Tuberculosis in swine. Channels of infection. It is stated that young animals belonging to the precocious breeds seem to be more liable to tuberculosis than others. In nine cases out of ten the animals are infected by ingestion. The pig easily becomes tuberculous when fed on material rich in tubercle bacteria. If pigs are fed on the refuse from dairies and cheese manufactories in districts where there is much

*The Federal meat inspection regulations state, that animals affected with "extensive or generalized tuberculosis" are to be condemned. 
tuberculosis in cattle or on tuberculous viscera they readily become infected. Infection through the respiratory tract, while it is certainly possible, seems to be rare. The piggeries where the refuse from butter and cheese factories is fed and those which adjoin abattoirs supply the majority of animals found on post-mortem to be tuberculous.

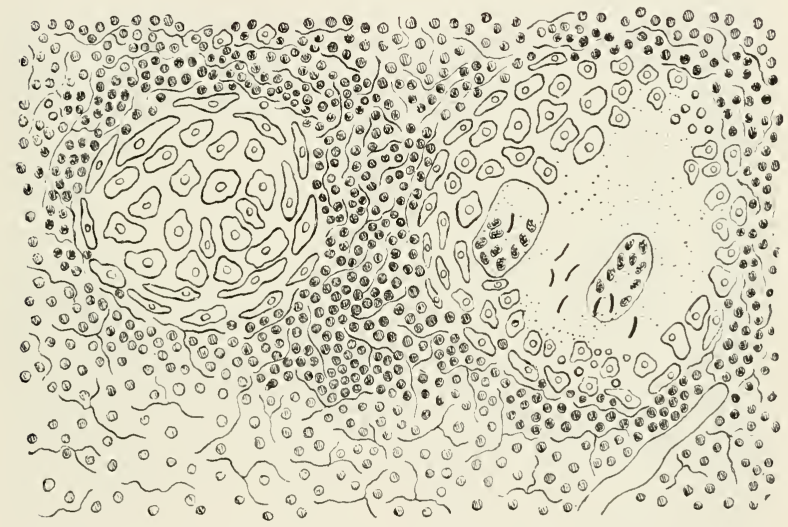

FIG. 3r. A drawing of a section of a very young tubercle in spleen (Thoma).

Ostertag has called special attention to this disease as existing among swine in certain parts of northern Denmark and Germany, where there was much tuberculosis in cattle, and where swine were fed upon the slime from creamery separators. Experiments show the possibility of infection by means of the sputum of tuberculous people.

In the cases which have come to our notice there is very strong evidence that the swine were infected by being fed upon milk from tuberculous cows. In one of these cases, the tuberculin test showed that a large number of the cows from which the milk was obtained were affected. 


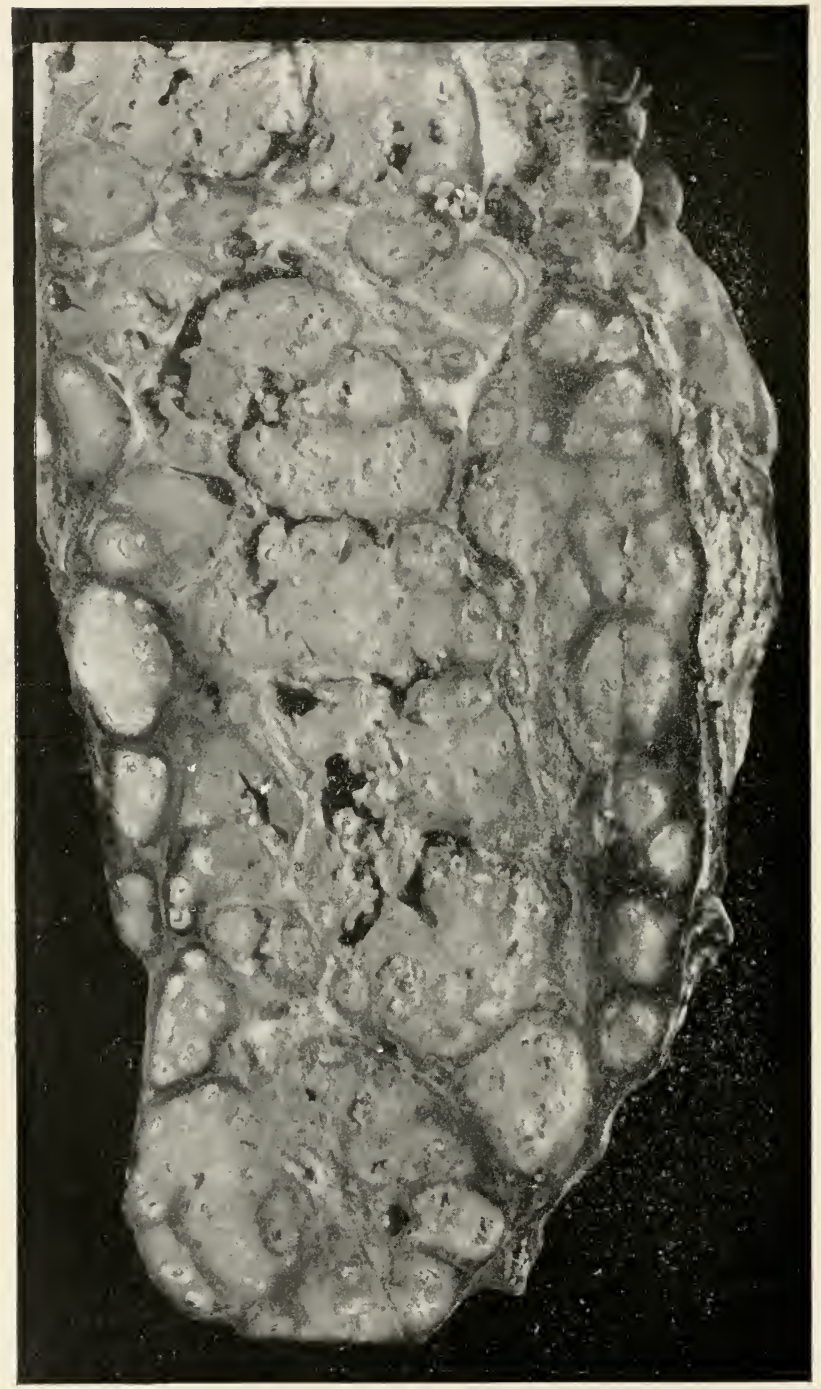

FIG. 32. Photograph of a section from anterior lobe of a tuberculous lung of a cow, showing rounded tubercular infiltration and calcified centers. (Law.) 
SII3. Symptoms. In most cases tuberculosis of the pig is first recognized at the abattoir. Sometimes, however, it causes local and general troubles, which vary according to the organ or system attacked. The following symptoms have been noted.

Its localization in the abdominal organs causes the arrest of fattening and the progressive wasting of the subject. The mucous membranes become pale; the hide becomes dirty ; there is usually either constipation or diarrhoea. The animal is in low spirits and remains buried in its straw for entire days ; the corkscrew of its tail is straightened; the abdomen is pendulous and the eyes sunken ; palpation of the abdomen is painful and may reveal more or less voluminous masses, due to the changes in the mesenteric glands. It is common to find glandular tumors in the submaxillary region at the level of or at the thoracic inlet. In this form the malady may last several months, but death supervenes rapidly if the lesions are generalized through the blood stream. Primary pulmonary tuberculosis is very rare but sooner or later lung lesions complicate abdominal tuberculosis, and betray theniselves at the outset by a short, dry, abortive cough and by difficult respiration. The cough soon becomes paroxysmal and painful and is often followed by romiting; the respiration becomes hurried and gradually painful and more difficult ; wasting is very rapid and death supervenes in a few weeks.

The scrofula of swine (glandular tuberculosis) usually shows itself by a puffing up of the face, which a careful examination shows to be lifted up by the subjacent glands, which are enlarged, indurated, still fairly mobile and free from heat or tenderness. The retro-pharyngeal, superior cervical, and sublingual glands usually take part in the lesion, forming a kind of necklace of unequal and knotty tumors, which extend from ear to ear, and become larger under the neck between the two of the lower jaw. Similar tumors may be developed at the rami, thoracic inlet, behind the shoulder or in the groin, which, as they increase in size, become harder and more adherent to the neighboring tissues. Sometimes, however, a slight fluctuation is perceptible; the tumor softens, then forms an abscess and 
discharges a small quantity of thick and grumous pus; but the glandular tumor does not disappear and the opening into the abscess remains for a long time as a fistula.

At the same time one may notice swellings of the bones, causing a true tuberculous arthritis when the lesions happen to be situated at the level of an epiphysis. Persistent lameness, fistulous wounds suppurating indefinitely, necrosis, caries, etc., are the complications of the lesions of the bone, the development of which is always extremely slow.

II4. Morbid anatomy. The manifestations of tuberculosis in swine, as suggested in the foregoing statement, are exceedingly interesting. Nocard finds the lesions to consist of niliary granulations which rapidly become caseous, as in cattle, but which more rarely contain calcareous salts. Generalization is common, in which case the viscera are thickly sprinkled with gray granulations which are translucent throughout, or opaque in their centers, and quite analogous to those found in tubercular lesions in other animals.

As the disease most often results from ingestion of the virus, the digestive apparatus and the corresponding lymphatic glands (submaxillary, parotid, pharyngeal, superior cervical, mesenteric, sublumbar, etc.) may be decidedly altered, while the other organs remain practically intact. Lesions of the small intestine and the cæcum are common and take the form of ulcers of the mucous membrane, of miliary nodules or of tuberculous infiltrations involving at once the mucous, the muscular, and subserous tissues. The lesions in the liver take the form either of miliary granulations, which are yellow and caseous and scattered in great numbers through the thickness of the organ, or else of rounded nodules which are yellowish white in color, varying in size from that of a pea to a hazel nut, and of a tough consistency. On section they appear sometimes to be firm, homogeneous and fibrous; sometimes softened in the center, but rarely infiltrated with calcareous salts. The peritoneum and the pleura are sometimes the seat of an eruption of fine granulations which remain in a state of niliary nodules. Lesions like those in the liver may exist in the lungs, but generally there is found in these organs au innumerable 
Plate III.
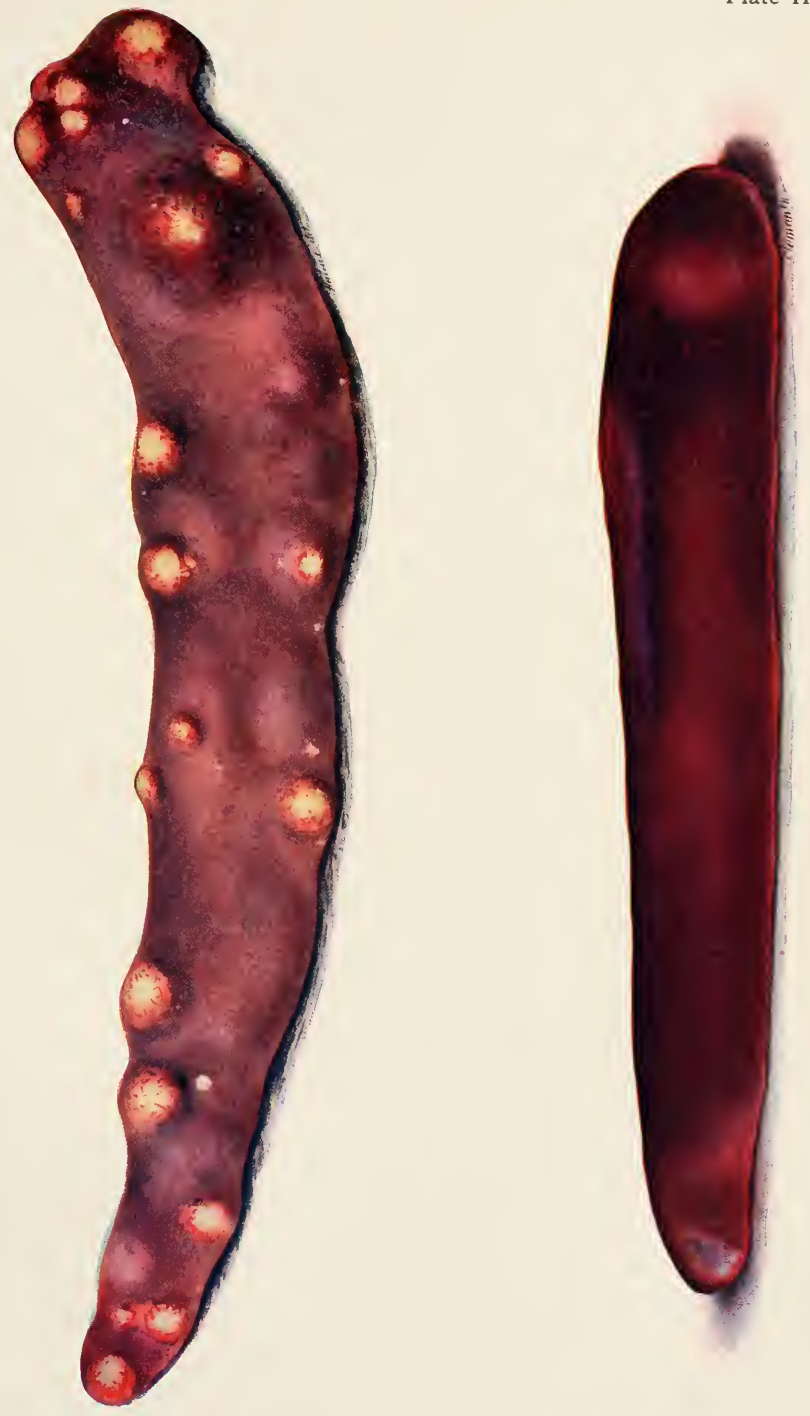

quantity of minute translucent, gray granulations, caused by generalization through the blood stream, in which case the liver, the spleen, the kidneys, the medulla of the bones, and the mammae are usually infiltrated with similar growths.

It is common to find lesions localized in one or several lymphatic glands. The tonsils and the pharyngeal or submaxillary glands are the ones most often affected. They become voluminous, hard and knotty, as they have undergone a true fibrous transformation and, consequently, difficult to cut. This is shown by the tissues creaking under the cutting instrument. In section they have the appearance of old fibrous tissue; here and there small yellow foci are seen of a softer consistency, almost caseous ; sometimes veritable purulent collections are found, either encysted or in communication with the exterior. If one submits the caseous or purulent matter to a bacteriological examination, tubercle bacteria are not usually found. The bacterium, however, is present and if this matter is inoculated into the peritoneal cavity or the cellular tissue of guinea pigs it will produce tuberculosis.

These chronic glandular lesions, with their very slow progress, have long been looked upon as constituting the scrofula of swine, and to scrofula was also assigned the tuberculous lesions of bones (ribs, vertebrae, articulations, shoulder blades, hip bones, etc. ) which are common in pigs, both young and old.

The older authors noted that the ancient scrofula was often accompanied by visceral tuberculosis, but they refused to admit the identity and even the relationship of the two affections.

The generalization of the disease especially in the muscular tissue is reported by several observers. Moulé calls attention to this peculiarity of the disease. Stockman shows that while the disease is ordinarily generalized, muscular lesions may exist in swine in the absence of generalization. Zschokke has called special attention to the localization of tubercular lesions in the head of swine, especially in the nares and brain.

Tuberculosis in the horse is rare although a total of many cases has been reported. Bang has collected twenty-nine cases. In Saxony .os per cent of the horses $(3,500)$ that were slaugh- 
tered were tuberculous. In this and most countries there are no reliable statistics respecting the extent of the disease in this species. M'Fadyean has pointed out the fact that in a considerable number of cases of equine tuberculosis, where the horses have been fed milk from tuberculous cows, the morbid anatomy differs but slightly from that in tuberculous cattle.

Sheep and other domestic animals are reported to suffer more or less extensively from this disease. All of the so-called tuberculosis in sheep and fowls which I have been able to examine proved not to be tuberculosis but animal parasitisms such as the "nodular disease of sheep" and "taeniasis" in fowls.

S I I5. Differential diagnosis. Tuberculosis is to be differentiated from actinomy cosis, glanders, and various parasitisms resulting in nodules largely in the walls of the intestine. In cattle the nodules are produced by an Oesopliagostoma, in sheep the nodules are caused by Oesophagostoma Columbianum Curtice. In chickens a nodular taeniasis of the intestine is not infrequently mistaken for tuberculosis. Abscesses and necrotic foci due to various agencies must also be distinguished from tubercular lesions.

In cases of actinomycosis, the ray fungus can usually be detected on a microscopic examination. Bact. mallei can be found either in cultures or by guinea pig inoculations in cases of glanders, and in parasitic diseases the specific animal parasite can be found if diligently sought. A careful study of the recent lesions, especially in the nodules caused by the animal parasites will show that they are not structurally like the tubercle as described above.

From the symptoms and morbid anatomy it is clear that hard and fast lines for diagnosing tuberculosis cannot be laid down. As a rule, the lesions are characteristic although there are many exceptions. In making a positive diagnosis, one must rely upon the discovery, microscopically, of the specific bacterium, the result of animal inoculation or the effect of tuberculin.

The tubercle bacteria can be found by making and properly staining cover-glass preparations from the tuberculous tissues or discharges in a certain number of cases. When these tuber- 
Plate IV.

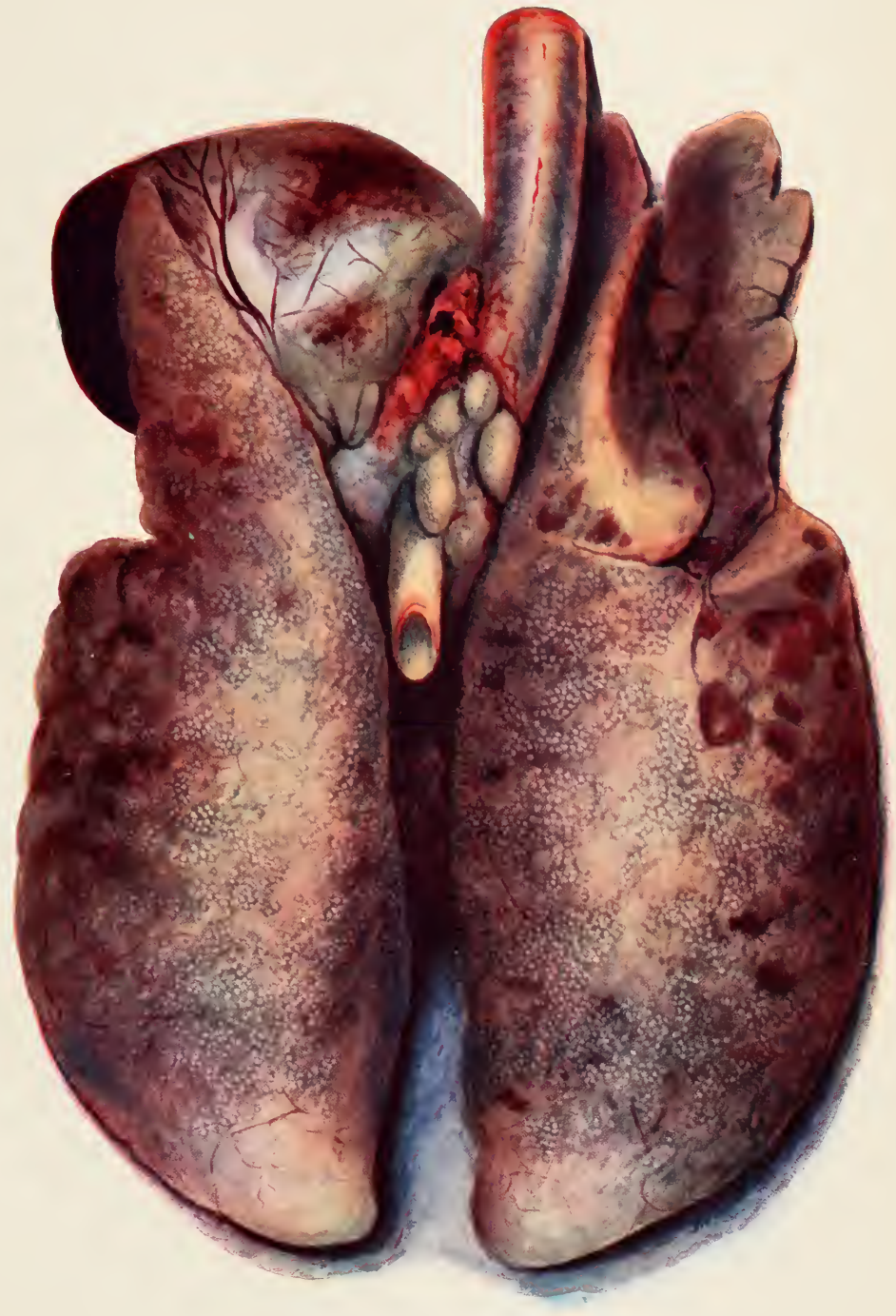

TUBERCULOUS LUNGS OF HOG. 


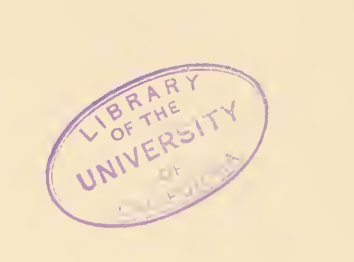


cular lesions open into the respiratory tract the specific bacteria can almost always be found in the expectoration. This is especially true in men and Ravenel has shown that it is often true in cattle. In old and in the very recent tuberculous lesions, it is not so easy to detect these organisms microscopically. When there is doubt the animal inoculation gives quite prompt results.

SII6. Animal inoculation for purposes of diagnosis. Guinea pigs are preferable, although rabbits may be used. With tuberculous tissue either of two methods may be employed. (I) A small piece (about the size of a pea or bean) of the tissue may be inserted under the skin by first making an incision with a sharp scalpel through the skin and superficial fascia, and then with a pair of fine forceps insert the bit of tissue well under the skin and close the opening with one or more sutures. (2) The tissue may be crushed in a mortar and thoroughly mixed with a few cubic centimeters of sterile water or bouillon and then injected with a hypodernic syringe. The needle should be of large calibre. If it is suspected milk it may be injected into the abdominal cavity. If the material is tuberculous and contains living tubercle bacteria, the death of the animal follows in from three weeks to four months. Usually the lymphatic glands in the groin and axilla are enlarged and often caseous. If a guinea pig is used, the liver, spleen, lungs and kidneys are liable to be affected, in the order named; if a rabbit, the lungs are often the first of the viscera to be attacked.

SII7. Tuberculin test. The tuberculin test is the best practically and in a large majority of tuberculous cases among animals and in man the only means of positively detecting the disease in the living individual.

Tuberculin. Tuberculin is the concentrated liquid, usually glycerinated bouillon, on which tubercle bacteria have grown until the products resulting from their multiplication have become imparted to the medium in sufficient quantity to inhibit their furtler development. It is not definitely determined just what these products are or just how they are elaborated. Briefly stated, the preparation of tuberculin consists in the following procedures, viz: 
(I) The preparation of the culture medium (glycerinated bouillon), distributing it in suitable flasks and inoculating it with the growth from a pure culture of tubercle bacteria.

(2) The flasks are placed in an incubator at a temperature of about $37^{\circ} \mathrm{C}$. where they remain until the growth ceases. The length of time necessary to accomplish this depends upon the age and condition of the culture from which the inoculations were made. From four to ten weeks are usually required.

(3) After the maximum growth is attained, the cultures are sterilized by heat, either by boiling in a closed water bath or heating to a higher temperature in an autoclav.

(4) After sterilization, the cultures are filtered to remove all of the dead bacteria, and then the filtrate is evaporated over a water bath to the desired degree.

(5) The concentrated liquid is passed through a Pasteur or Berkefelter filter, standardized, bottled and labeled for distribution. It should be perfectly clear although its color may vary. If it is cloudy it should be rejected.

It will be seen from the method of preparation that tuberculin cannot possibly contain living tubercle bacteria. It is heated on two occasions to a temperature and for a length of time far in excess of that required to destroy them besides being passed through a filter capable of removing all bacteria.

The original tuberculin or lymph of Koch was concentrated to one-tenth of the volume of the saturated culture. This gave a thick, syrupy liquid owing to the presence of the glycerine. The diagnostic dose which came to be recommended for cattle of medium weight was 0.25 c.c. On account of its consistency as well as the minuteness of the dose, it was found to be practicable to dilute this quantity with seven parts of a diluent. A weak solution of carbolic acid was ordinarily used. The difficulties and the danger of contanination involved in making the dilutions in the field led to the method of diluting the tuberculin in the laboratory before sending it out. This has been the practice of the Bureau of Animal Industry for a number of years. Equally as good results are obtained by concentrating the saturated culture to the point where 2c.c. 
contains an equivalent of the 0.25 c.c. of the highly concentrated lymph. This process avoids the necessity of dilutions and, with the addition of a few drops of carbolic acid, the weaker solution keeps perfectly.

Tuberculin in the dose necessary to bring ont its diagnostic effect is absolutely harmless to a healthy animal. Thousands of observations which have been reported assure us of this fact. Tuberculin is in daily use in every state in the Union, in Canada and in every country in Europe, yet so far as can be learned not a single case of injury following its use in healthy cattle has been reported. In the tuberculous animal it produces a rise of temperature which, within certain limits, follows a definite course usually terminating in from i 8 to 24 hours after the injection. The temperature usually begins to rise in about eight hours giving a steady but quite rapid elevation for from I to 3 hours. A continuous high elevation for from 2 to 4 hours, possibly longer, and a gradual decline. This is practically constant, be the raise moderate or extreme, In addition to the elevation in temperature there is sometimes a marked nervous chill. Why we get this reaction* is not positively determined.

*Trudeau (Johns Hopkins Hospital Bulletin, July, 1899) gives the following summary of the mechanism of the tuberculin reaction. "The most generally accepted theory at present in regard to it is, briefly, the small dose of tuberculin injected is a partly specific irritant both to tuberculous foci and to the susceptible organism in general. It produces intense hyperaemia of all tuberculous tissue in the body (local reaction), and as the result of this hyperaemia much toxin stored up in the tubercles themselves is thrown into the general circulation and produces fever and characteristic symptoms which go to make up what is termed 'a general reaction.' That these poisons stirred up in the tubercles are in part at least derived from the dead or weakened bacilli has been shown by the experiments of Babes and Proca, who found that if two sets of rabbits be injected with equal quantities of living and dead bacilli, the latter react to the tuberculin test at a much earlier period than those inoculated with living germs. This hypothesis that the general reaction is brought about by toxins already stored up in the tuberculous lesions and exploded as it were by the hyperaemia produced about these lesions as the result of the test injection of tuberculin, is borne out by the fact that a greater amount of albumose can be recovered from the evaporated urine col- 
Applying the tuberculin test. In brief, the method for applying the tuberculin test in cattle is as follows:

I. The normal temperature of the animal to be tested must be determined. It is recommended that it be taken hourly or every two hours for the day preceding the test.

2. The tuberculin is injected subcutaneously in the side of the neck. Care must be taken that the syringe is sterile and the site of injection should be disinfected. The size of the dose depends upon the preparation of tuberculin.

3. Beginning 6 or 8 hours after the injection, the temperature should be taken hourly or at least every two hours for fully three-fourths of a day.

4. During the time of testing, the cattie should be kept quiet, free from all exposure, and fed normally.

5. In case of reaction, there should be a rise of at least I. $5^{\circ} \mathrm{F}$. above the maximum individual normal temperature as determined on the preceding day. The elevation should come on gradually, remaining practically at its fastigium for a few hours and gradually subside. Erratic elevations of short duration are to be excluded. In cases of doubt the animals should be retested.

6. Animals advanced in pregnancy and those known to be suffering from any disease or in oestrum should be excluded. All methods of treatment including exposure to cold, kind of food and drink which would tend to modify the temperature should be avoided. Animals in which the disease is well advanced sometimes fail to react.

7. The dose should vary to correspond with the weight of the animal. In cases of a second test, within a few days, the quantity of tuberculin injected should be larger than for the first test.

lected during the reaction than was contained in the test injection; also by clinical observations which indicate that patients suffering from localized surgical tuberculous processes of limited extent, and where the vascular supply to the part is limited, require a larger test injection to produce the reaction than those who have extensive or scattered visceral lesions in highly vascular organs like the lungs." The reader is referred to this paper for a careful consideration of the rexed questions relating to tuberculosis. 
In cattle there is a marked variation in the normal daily temperature. A fluctuation of two or even three degrees within $2+$ hours is frequently found. Cold water when drunk in considerable quantities lowers the temperature from two to four degrees. A temporary excitement usually causes an elevation of from I to $\mathrm{I} .5^{\circ} \mathrm{F}$. There are also marked variations in the temperature of the same animal on consecutive days. The temperature at 12 noon and 12 midnight are often the same. In some cases the maximum elevation for the day occurs near midnight and on the following day the minimum temperature appears at that time. It is not uncommon for the maximum temperature to occur twice in the same day and occasionally several times within the twenty-four hours. There are marked individual variations in the effect of ordinary conditions upon the temperature such as food, excitement or temperature of the air. A hot spell causes a rise of two and in some cases four degrees. The average temperature of the animals in three herds tested by Howe and Ryder were IO2. $5^{\circ}$, IO2. $6^{\circ}$ and $10 I^{\circ} \mathrm{F}$. respectively.

In a well kept Government herd that was tested with tuberculin, the temperature of part of the animals was taken hourly for 24 and part of them for 16 hours preceding the injection. An examina-

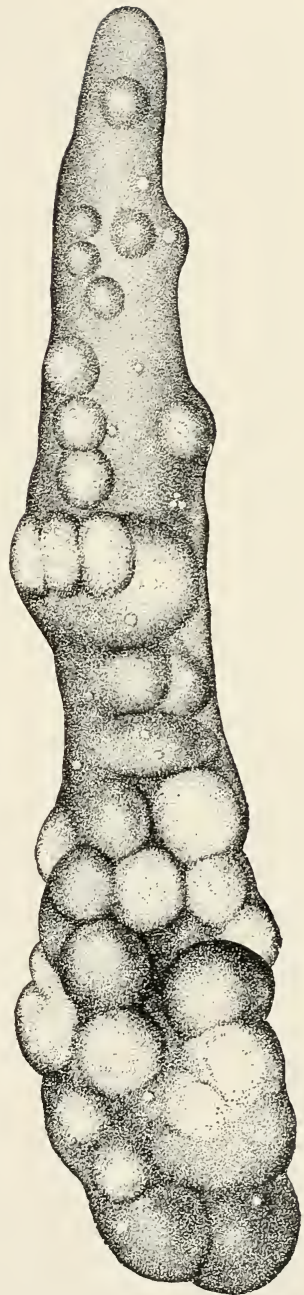

Fig. 33. Tuberculous spleen from a pig. (Law.) 
tion of the records* shows the average daily variation of 20 animals in which the temperature was taken for 24 hours to be $2.31^{\circ} \mathrm{F}$. The maximum individual variation in a single day was $4.3^{\circ} \mathrm{F}$, the minimum $0.5^{\circ} \mathrm{F}$. In 25 other animals where the temperature was taken for 16 hours, the average variation was I. $79^{\circ} \mathrm{F}$. In these the maximum variation was $3.2^{\circ} \mathrm{F}$, the minimum $0.6^{\circ} \mathrm{F}$. Ten healthy auimals (did not react to tuberculin) in the same herd gave an average variation of $2.08^{\circ} \mathrm{F}$. In these the maximum daily variation was $4 . \mathrm{I}^{\circ} \mathrm{F}$., the minimum $\mathrm{I}^{\circ} \mathrm{F}$. The lowest temperature was usually, but not invariably, in the morning and the highest in the afternoon or evening. I have appended the records of the temperature of two of these animals.

THE INITIAL, TEMPERATURE OF TWO COWS, WITH RATE OF PULSF AND NUMBER OF RESPIRATIONS PER MINUTE.

\begin{tabular}{|c|c|c|c|c|c|c|c|}
\hline \multirow{2}{*}{\multicolumn{2}{|c|}{ Hour }} & \multicolumn{2}{|c|}{ Cow, No. I } & & \multicolumn{2}{|c|}{ Cow, No. 2} & \multirow[b]{2}{*}{ Resp. } \\
\hline & & $\begin{array}{l}\text { Tempera- } \\
\text { ture }\end{array}$ & Pulse & Resp. & $\begin{array}{l}\text { Tempera- } \\
\text { ture }\end{array}$ & Pulse & \\
\hline 9 & A. $\mathrm{M}$. & 99.8 & 48 & IS & 98.6 & $4^{8}$ & I5 \\
\hline 10 & ". & 99.5 & 66 & IS & 98.6 & 60 & I 5 \\
\hline I I & " & 99.0 & 60 & I 5 & 99.0 & 60 & I5 \\
\hline I 2 & " & I00. 8 & $5+$ & 15 & 99.4 & 54 & I 5 \\
\hline I & P. M. & IOI. 4 & 54 & 15 & IOO.O & 54 & IS \\
\hline 2 & 6 & IOI. 6 & 48 & I 5 & 100.2 & 54 & IS \\
\hline 3 & "“ & 102.0 & 60 & 24 & 100.4 & 72 & 24 \\
\hline 4 & "، & $\mathrm{IO}_{3} .0$ & 66 & 24 & 102.7 & 72 & 24 \\
\hline 5 & “ & 103.3 & 66 & 24 & 1028 & 72 & 27 \\
\hline 6 & " & IO3. I & 57 & IS & 103.0 & 60 & 27 \\
\hline 7 & "، & 102.2 & 60 & 20 & 102.4 & 60 & 24 \\
\hline S & "، & 103.0 & 56 & I6 & IO2.O & 60 & 24 \\
\hline 9 & “ & I03. 1 & 52 & 24 & 102.2 & $5^{\circ}$ & 24 \\
\hline IO & “ & I02. 5 & 60 & 20 & 102.0 & 50 & IS \\
\hline I I & ، & IO2.5 & 60 & 20 & 102.0 & 60 & 20 \\
\hline & midnight & IO2 4 & $5^{6}$ & I 6 & 101. 6 & 54 & 20 \\
\hline & A. $\mathrm{M}$ & IOI.S & 60 & 20 & IOI. 4 & 58 & 24 \\
\hline 2 & “ & 102.0 & 64 & IS & $\mathrm{IO} 2.2$ & $5^{8}$ & I8 \\
\hline 3 & " & 102.0 & 60 & IS & IOI. 6 & $5^{8}$ & 18 \\
\hline 4 & " & 102.2 & 54 & 24 & IOI. 5 & 60 & 24 \\
\hline 5 & “" & I01. 6 & 56 & 24 & 102.0 & 60 & I 8 \\
\hline 6 & " & IOI.S & 60 & IS & IO2. 2 & 60 & 20 \\
\hline & " & IO2. 5 & 56 & I 6 & 103.2 & 60 & I 8 \\
\hline
\end{tabular}

*Bulletin No. 7, Bureau of Animal Industry, U. S. Department of Agriculture, Washington, D. C. The tests were made by Drs. F. L. Kilborne and E. C. Schroeder, under the direction of Dr. Theobald Smith. 
In view of these normal temperature variations, which often exceed the tuberculin reaction, it is obvious that before applying the test the normal temperature of the animals should be approximately determined and that when they are being subjected to the test they should be cautiously protected, otherwise the comparatively slight elevation necessary to detect the disease may be disguised.

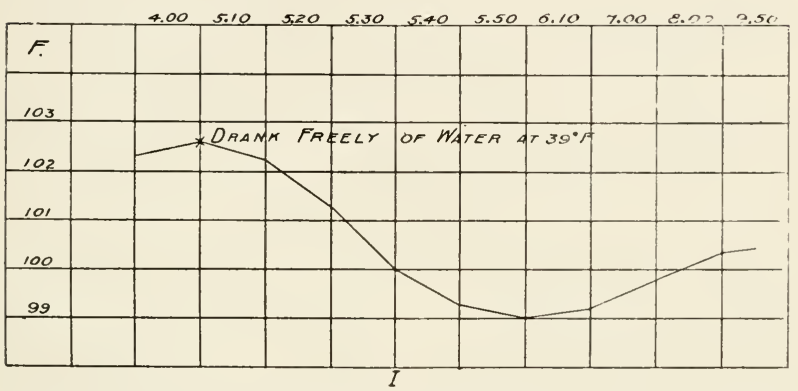

FIG. 34. Chart showing the effect of drinking cold water upon the temperature of a cow.

As the reaction seems to be the result of an affinity existing between the tuberculin and the living tuberculous lesion. it is natural to suppose that when the two are brought together in the same animal it would invariably take place. Experience has shown that it almost always does. It is important to understand, as far as possible, the reason for the exceptions and the extent to which they occur. The reported failures of the tuberculin tests fall into two distinct classes :

( 1 ) When there is a reaction and no disease is found. In explaining this alleged error, the records of the cases which have come to my attention have been so deficient in data concerning the normal temperature variation of the animals, and the incompleteness of the post-mortem examinations, that it seems possible for the error to rest with the observer quite as much as with the tuberculin. Unfortunately we are as yet unable to determine, by the reaction, the extent of the disease, 
so that a beginning lesion no larger than a walnut may cause a pronounced rise of temperature and such a tubercle may be difficult to find, especially if located in the marrow of some bone.

(2) Where there is no reaction and the disease exists. It is generally admitted that advanced cases often fail to react, but here the test itself is of little importance as the disease can be detected by the clinition on physical examination. When, however, tuberculin is carefully prepared and scientifically administered, these exceptions are exceedingly rare.

The practical value of tuberculin, however, lies in its efficiency in the arts of comparative and sanitary medicine. It is in the practical application that difficulties are encountered. The nuany details and precautions enumerated as absolutely

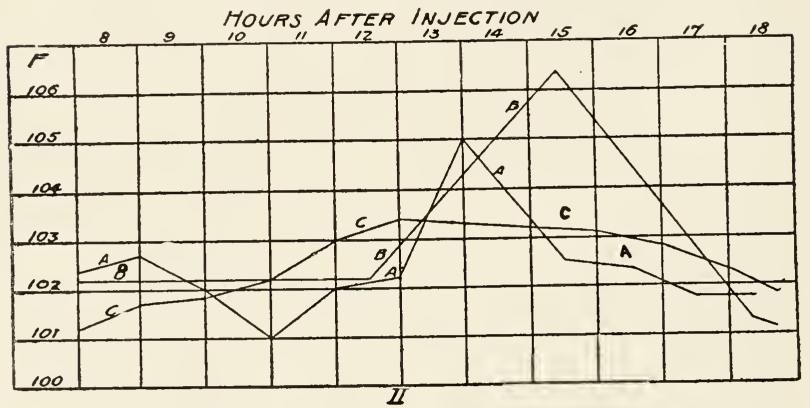

FIG. 35. Irregular temperatures following tuberculin that are not caused by tuberculosis. (Curtice.)

essential to the best results are often considered too tedious and time consuming, and consequently the practice has come too generally into vogue of neglecting or ignoring many of the precautions. In order that the test may be practicable, some practitioners resort to a shorter method of procedure even at the risk of an occasional error.

S I 8 . Prevention. Tuberculosis, like other infectious diseases, can be very largely prevented. To accomplish this it is necessary to keep tuberculous animals from entering the 
healthy herds. If they are admitted and later the fact is discovered, it is necessary to remove them and to thoroughly disinfect the stable. In eliminating the disease from a herd, by nineans of the tuberculin test, it is necessary to retest the

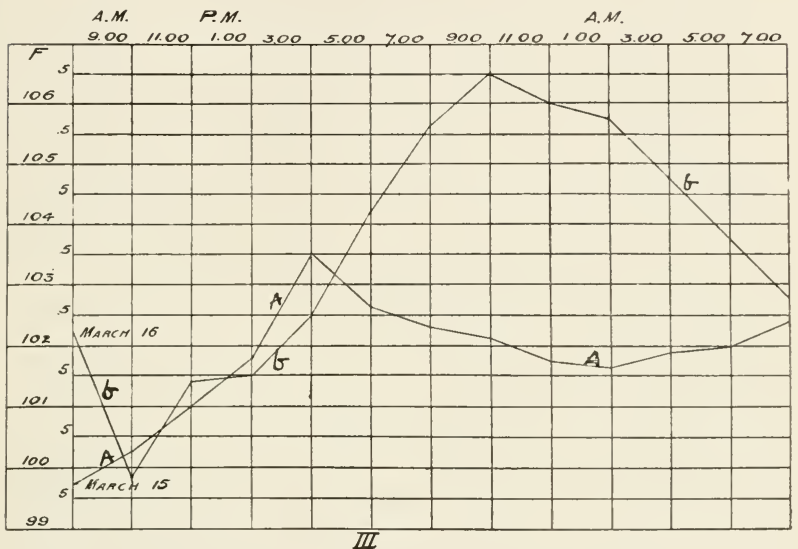

Fig. 36. Temperature curve of a tuberculous cow for 48 hours. The line A.A. shows temperature for 27 hours preceding the injection of tuberculin which was injected at $9 a . m$., March $16 ; b . b$. b. shows the temperature for the $2+$ hours after the tuberculin injection.

non-reacting animals after six months or a year has passed in order to find any case that might have been infected, but in which the disease had not begun to derelop, at the time of the first test.

\section{REFERENCES.}

I. ADAMI. On the significance of bovine tuberculosis and its eradication and prevention in Canada. Canadian Jour. of Medicine and Surgery. Dec. 1899

2. CuRTice The detection of tuberculosis in cattle. Annual Keport, Bureau of Animal Industry, U. S. Dept. Agric. ${ }^{8} 895^{-96 .}$

3. Kосн. The etiology of tuberculosis. Mitt. aus dem. Kaiserl. Gesundheitsamte. Vol. II. ISS4. Translated in Vol. CXV. New Sydenham Society.

4. Kосн. The combating of tuberculosis in the light of the experience that has been gained in the successful combating of other infectious diseases. Am. Vet. Review. Vol. XXV. No.6. Sept. Igor. 
5. MOORE AND DAwson. Tuberculosis in swine, the nature of the disease with a report of three cases. Annual Report, Bureau of Animal Industry, U. S. Dept. Agric. IS95-96.

6. MOORE, The preparation of tuberculin, its value as a diagnostic agent, and remarks on the human and bovine tubercle bacilli. Trans. of the Med. Society of the State of $N . Y .1900$.

7. NocARD. The animal Tuberculoses. New York.

S. Pearson. The Pennsylvania plan for controlling tuberculosis of cattle. Proc. Am. Vet. Med. Assn. iSg9.

9. Pearson. Tuberculosis in cattle and the Penn. plan of its repression. Bulletin 75 Penn. Dept. of Agr. 1901.

IO. PEARSOx. The repression of tuberculosis of cattle by sanitation. Bulletin 7t. Penn. Dept. of Agric. 1901.

if. RAVENEL. The dissemination of tubercle bacilli by cows in coughing a possible source of contagion. Lniv. of Penn. Med. Magazine. Nov. Igco.

12. RAVENEL. The comparative virulence of the tubercle bacillus from human and bovine sources. L'niv. of Penn. Med. Bul. Sept., Igor.

13. RAVENEL The intercommunicability of human and bovine tuberculosis. The Univ. of Penn. Medical Bulletin. May. I902.

I4 REPP. Transmission of tuberculosis through meat and milk. Anerican Medicine. Oct. 6, Nov. 2. Igor.

15. SALMoN. Legislation with reference to bovine tuberculosis. Bulletin 2S. IT.S. Dept. of Agr. Bureau of Animal Industyy. 1901.

16. Salion. The tuberculin test of imported cattle. Bulletin 32. Bureau of Animal Industry, U.S. Dept. of Agr: 1901.

17. Surth. Investigations concerning bovine tuberculosis with special reference to diagnosis and prevention. (Pathological part). Bulletin No. 7., U.S. Dept. of Agr. Bur. of Animal Industry. IS94.

IS. Sirrth. A comparative study of bovine tubercle bacilli and of human bacilli from sputum. The Jour. of E.rper. Med. Vol. III. ISgs.

19. SuITH. The thermal death point of tubercle bacilli in milk and some other fluids. Jour. Experimental Med. Vol. IV. No. 2. IS9g.

The following bulletins on tuberculosis have been issued from the various State Agricultural Experiment Stations:

Bitrisg. Bovine tuberculosis in Indiana. (Purdue). Bul. 63. Ind. I 996 .

BREIVER. Tuberculosis. Bul. 4I. Utah. IS95.

CARY. Bovine tuberculosis. Bul. 67. Alabama. IS95.

Fischer. Bovine tuberculosis. Bul. 79. Kansas. 1898.

GraNgE. Tuberculosis. Bul. I33. Mich. 1896.

HILLS AND RICH. Bovine tuberculosis. Bul. 42. Vermont. $1 S 94$.

LAw. Tuberculosis in relation to animal industry and public health. Bul. 65. (Cornell.) New York. IS94.

LAw. Experiments with tuberculin on non-tuberculons cows. Bul. 82. (Cornell.) New York. IS94.

LAw. Tuberculosis in cattle and its control. Bul. 150. Cornell. I $89 \mathrm{~S}$.

MARSHALL. A study of normal temperatures and the tuberculin test. Bul. 159. Michigan. I 89 S.

MARSHALL. Killing the tubercle bacilli in milk. Bul. 173. Michigan. IS99.

Mavo. Some diseases of cattle, Texas itch, blackleg, tuberculosis, Texas fever. Bul. 69. Kansas. IS97.

NeLson. On the use of Koch's lymph in the diagnosis of tuberculosis. Report of the biologist. New Jersey. IS93. 
NeLson. Experimental studies of the Koch test for tuberculosis. New Jersey. I 895 .

NELSON. The suppression and prevention of tubercnlosis of cattle and its relation to human consumption. Bul. I18. New Jersey. I896.

Nesom. Tuberculosis of cattle. Bul. 50. S. C. Igoo.

Pearson. Tuberculosis of cattle. Bul. 29. Pein. IS94.

RUSSELL. Tuberculosis and the tuberculin test. Bul. 40. Wisconsin. I 1894

RUSSELL. The history of a tuberculous herd of cows. Bul. 78 . Wisconsin. 1899 .

RUSSELL aND Hastings. Bovine tuberculosis in Wisconsin. Bul. 84. Wisconsin. I901.

STALKER AND NILES. Investigation of bovine tuberculosis with special reference to its existence in Iowa. Bul. 29. Iowa. IS95.

THORNE. Bovine tuberculosis. Bul. IOS. Ohio. 1899,

Williamson and EMERY. Tuberculosis and its prevention. Bul. 117. N. C. IS95.

\section{OVINE CASEOUS LYMPH-ADENITIS. (PSEUDO TUBER- CULOSIS IN SHEEP.)}

SIrg. Characterization. Caseous lymph-adenitis is a disease of adult sheep which until recently was designated as pseudo-tuberculosis. It has been characterized by an enlargement of one or more lymphatic glands which contain foci of a greenish-yellow, caseous or purulent substance. It is rarely found in young animals. The mortality is very low due, perhaps, to the fact that the sheep are slaughtered before the disease has time to develop. It does not occur in epizoötic form although it is more prevalent in certain localities than in others.

\$ r2o. History. The name "Ovine Caseous Lymph Adenitis' was proposed by Nörgaard and Mohler in I 899. These writers found the lesions and the accompanying microorganism to correspond with those described by Preisz and Guinard in I 89 I as pseudo-tuberculosis. The bacterium was fully described by Preisz in I 894 . The organism has been found and identified from a large variety of lesions in a number of species of animals. It appears that at least many of the cases of lymphatic gland enlargement heretofore called pseudo-tuberculosis in sheep belong to this disease. Cherry and Bull describe it as caseous lymphatic glands and Sivori as caseous broncho-pneumonia ; the bacterium of Preisz being found as the probable cause in each case. 
I21. Geographical distribution. In the United States this disease is quite common in certain districts in the western and southwestern states. It exists in South America, Australia and Europe.

Sivori found that Io per cent of the old sheep killed in Buenos Ayres were affected. The prevalence of the disease in the United States, as indicated by the reports of the federal meat inspectors, show that of 16,000 , o0o sheep slaughtered in Chicago, Kansas City and South Omaha 3,236 were condemned for caseous lymph adenitis or lesions which might be confounded with it. It is reported by an inspector from Los Angeles that of 950 sheep coming from an infected district, 82 were suffering from lymph adenitis.

I22. Etiology. Caseous lymph adenitis is caused by a specific organism. It appears to be the one described first by Preisz as the bacillus of psendo-tuberculosis. Its description shows it to vary in size to such a degree that its polymorphism is said to be characteristic. It is nonmotile and hence belongs to the genus Bacterizm. It is aërobic,

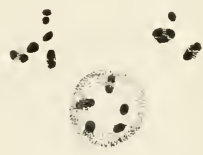

FIG. 37. Bacterium of Preisz. facultative anaërobic, it stains readily and does not produce spores. It develops readily on agar when this medium is inoculated from the caseous material from the affected glands. It is pathogenic for mice, guinea pigs and rabbits.

$\$$ I23. Symptoms. In the majority of cases no symptoms of any importance are observed in the affected animals during life. The course of the clisease is that of a chronic affection and the pathological changes develop so slowly that no general or local interference with the health of the affected animals are observed, in lambs and sheep that are bred and raised for mutton, and which are marketed before they are two years old. Only in breeding ewes and wethers does the disease adrance to a degree which makes it clinically recognizable without the aid of manipulation. The affected animals upon examination show an enlargement of one or more of the superficial glauds, the precrural and the sub-scapular glands being most often involved. The animals thus affected 
appear in every other respect to be in perfect health. In the older animals-the wethers and breeding ewes-the same glands may be enlarged to a considerable degree reaching the size of a hens egg or even larger. Some of these sheep may show a certain degree of unthriftiness or even emaciation. The disease is found in its most advanced stages in the older ewes, which is probably due to the fact that the wethers are generally disposed of before they are three years old, while a good breeding ewe is frequently retained for seven or eight years. In such old animals the superficial lymphatic glands may be enlarged to such a degree as to interfere with locomotion, while the deeper seated glands and those of the body cavities are similarly affected. In the advanced cases the lesions often become disseninated by metastasis to the principal organs of the body. In such cases the disease may assume the appearance of chronic broncho-pneumonia or pleurisy, with occasional cough, slight dyspnoea and increasing emaciation and anæmia. The course of the disease is exceedingly slow. For this reason owners of affected flocks are often totally ignorant of the presence of the disease. This fact renders it very difficult to obtain reliable information regarding its prevalence save from the statistics obtained from the slaughter houses. A majority of the inspectors have until recently classified the lesions either as tuberculosis, pyemia or abscesses. In response to inquirics it was found that the majority of cases which had been condemned under these headings were undoubtedly caseous lymph-adenitis. Several thousand cases are annually observed in the slaughter houses of the United States, but only a fraction of these are advanced to a degree that would warrant a total condemnation of the carcasses. Meat inspectors agree that lambs are very rarely affected, and that the progress of the morbid changes in the majority of cases is coördinate with the age of the animal.

\$24. Morbid anatomy. The principal lesions are confined, according to the various descriptions, especially that by Nörgaard and Mohler, to the lymphatic glands. In many cases only a single gland is affected. The relative frequency with which the various glands become the seat of the lesions 
may be given as follows: prescapular, precrural, superficial inguinal, bronchial, mediastinal, sub-lumbar, deep inguinal, and scrotal. Rarely the suprasternal and mesenteric glands are affected. Sivori mentions the mesenteric glands among those frequently affected. He fails, however, to mention the mesenteric glands as the seat of lesions in the detailed description of twelve typical cases of caseous broncho-pneumonia caused by the bacillus of Preisz.

When first invaded by the bacterium, the adenoid tissue becomes hyperplastic and the gland enlarges to several times its original size. On section, the surface is found to be watery but otherwise the tissue retains its normal appearance. This is followed by the formation of various centers of degeneration which show coucentric layers and gradually become confluent. Finally, the total volume of the gland is transformed into a homogeneous caseous mass. At the same time the distended capsule increases in thickness and forms a sac which confines the semifluid, grumous mass. In rare instances the sac ruptures and when close to the surface the contents will be discharged. Under ordinary circumstances, the caseous contents become cohesive and sticky and of the consistency of putty. In very old cases the mass becomes dry and mealy with little or no tendency to calcification. The greenish yellow color of the caseous mass, which is stated to be most characteristic, closely resembles the contents of the intestinal nodules produced by Oesophagostoma Columbianum. In very advanced cases, as for instance those of old breeding ewes, the internal organs may contain lesions which microscopically resemble those of tuberculosis. The lungs may be studded with small nodules the size of a pea, the spleen, liver and in rare instances the kidneys also may contain one or more foci of the same character, namely, a mass of greenish yellow material, surrounded by a firm, fibrous sac. There seems, however, to be a distinct line of demarcation between the affected and the healthy tissue. There is no indication of an inflammatory zone. The bronchial and the mediastinal glands may be affected to a considerable extent without any lesions being found in the lungs. In some cases the lungs are extensively involved. 
The lesions consist of nodules varying in size from that of a millet seed to that of a walnut. This condition is, as a rule, accompanied by a chronic pleurisy with extensive adhesions and also effusions into the plural cavities.

In the liver the lesions consist largely of nodules composed like those in the lymphatic glands, of a firm white fibrous sac containing a greenish-yellow, cheesy mass of varying consistency. Cases have been reported, however, where the entire organ was filled with miliary nodules.

The kidneys are rarely affected but when they are the lesions assume the same characteristic appearance of a firm walled abscess protruding on the surface of the organ. As a rule, only one or two such foci are observed in each case.

A histological examination of tissue containing miliary or sub-miliary nodules, show them to be composed chiefly of leucocytes and nucleated round cells, the greater part of which are irregular in shape, especially toward the center where many of them are transformed into a granular detritus. Among the cells singly or arranged in clumps, are seen the short plump bacteria which stain irregularly. The shape varies considerably from oval or oblong to dumb-bell and club shape.

The bacteria are frequently seen within the degenerated leucocytes, the destruction of which is due, according to Preisz, to the specific chemical products elaborated by these microorganisms.

The microscopic appearance is somewhat similar in all the lesions whether located in the lymph glands, lungs, liver, kidneys or spleen. In the lungs the histological picture resembles that of broncho-pneumonia. In the liver the lesions originate in the portal capillaries, where the bacteria cause a proliferation of the endothelial cells, which, together with the accumulation of leucocytes and red blood corpuscles, cause the obliteration of the vessels. In no case have giant cells been observed. The surrounding hepatic cells become swollen, then granular and finally they undergo atrophy, leaving open spaces between them. Numerous round cells appear in the periphery of the nodules which gradually undergo a connective 
tissue metamorphosis and become organized into an encapsulating membrane.

When a miliary nodule from the liver of an experimental animal which has been destroyed three weeks after inoculation is examined microscopically the following picture is observed. A caseous center composed of an amorphous material that does not take any of the ordinary stains. Surrounding the center may be seen numerous leucocytes more or less degenerated and frequently containing one or more bacteria while clumps of these organisms are scattered among them. External to this is a dense round cell infiltration the peripheral zone of which is undergoing connective tissue formation thus serving as a line of demarcation between the atrophied liver cells and the central cell mass. The process then repeats itself until a connective tissue barrier strong enough to encapsulate the central part of the nodule and prevent its further growth is obtained. The nodules in the kidneys and lungs present a similar microscopic appearance, excepting that the foci in the lungs are more regular on account of the catarrhal inflammation that accompanies the reaction of the surrounding tissue. The center contains a dense mass of disintegrated cell structures composed of the desquamated and proliferated epithelial cells, degenerated leucocytes and round cells. In experimental animals which succumb quickly to an intravenous injection of virulent material, the lung tissue immediately surrounding the nodules is frequently seen to be hepatized.

I 25. Differential diagnosis. This specific lymphatic affection is to be differentiated from, (I) other infections which may cause enlargement or suppuration of lymph glands, and (2) tuberculosis. If the diagnosis cannot be made from the gross appearance of the lesions a bacteriological examination will be necessary. The fact should be kept in mind that tuberculosis in sheep is very rare. With lymph-adenitis, cultures in ordinary media will give a growth of the bacterium of Preisz. With tuberculosis the result would be negative. (See tuberculosis) It is important not to confuse the nodular disease of sheep's intestines with this affection. The location 
of the lesions in the walls of the intestine will be quite sufficient to determine the nodular disease.

\section{REFERENCES.}

I. CherRy AND BUl. Caseous lymphatic glands (pseudo-tuherculosis) in sheep. The Veterinarian. Vol. LXXII. p. 523.

2. EBERTH, C. J. Bacilläre Nekrose der Leber. Virchow's Archiv., Bd. C. (IS85) p. 23.

3. NörgaARD AND Mohrer. The nature, cause, and economic importance of ovine caseous lymph-adenitis. Sixteenth Annual Report, Bureau of Animal Industry. 1899. p. 63 S. (Full bibliography).

4. Preisz AND Guinard. Pseudo-tuberculose chez le mouton. Journ. de med. de vet. et de zoötech. ser. 3, Vol XVI. (isgi) p. 563 .

5. PREISZ. Recherches comparatives sur les psendotuberculoses bacillaires et une nouvelle espec de psendotuberculose. Ann. de l'Inst. Pasteur, Vol. VIII. (IS94) p. 23 I.

6. Sivori. Sur une broncho-pnemmonie caseouse du mouton, causee par le bacille de Nocard-Preisz. Rev. de med. vet. ser. 8, Vol. VI. (1899) p. 657 .

\section{ASTHENIA IN FOWLS AND PIGEONS.}

$\checkmark$ I 26. Characterization. This is a disease especially of chickens and pigeons in which there is marked emaciation and a failure to take on flesh even when fed on the most nourishing food. Because of this, the disease has received the popular name of "going light."

I27. History. Although this condition or disease has been recognized for a long time it seems to liave been first described in r 898 by Dawson. He gives a brief account of the symptoms, morbid anatomy, etiology and a somewhat extended description of the specific organism which he isolated from the diseased chickens. The writer has studied this affection in pigeons but did not succeed in finding the organism isolated by Dawson.

I 28. Etiology. Dawson found this disease to be due to the presence of a certain species of bacterium which he obtained in pure culture from the duodenal contents. He described it as Bacterium asthenia. This organism varies from $\mathrm{I}$ to $\mathrm{I} .3 \mu$ in length and about $0.5 \mu$ in width with rounded ends. It is reported to possess the peculiarity of vegetating in temperatures varying from 50 to

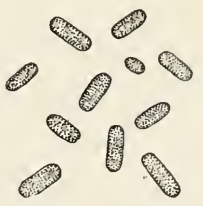

Fig. $3^{8 .}$ Bacterium asthenic. (Dawson.) 
I $20^{\circ} \mathrm{F}$. It is fatal to rabbits within 24 hours when inoculated into the abdominal carity with 0.5 c. c. of a bouillon culture. Chickens inoculated with this organism remained well.

I29. Symptoms. The only symptoms which seem to be in evidence are the gradual loss of flesh and an exceedingly good appetite. It is reported by certain pigeon fanciers concerning pigeons and the fact is reiterated by Dawson, that the disease is an exceedingly chronic one, often extending over a period of several months but usually terminating in death. In the cases reported, the fowls were well kept and given an abundance of nourishing food. There seems to be an inability on the part of the affected animal to assimilate nourishment.

\$ 130. Morbid anatomy. The most conspicuous lesion is extreme emaciation. According to Dawson the mucosa of the duodenum contains areas in which the walls are deeply reddened and in which the contents are of a mucoid substance. The writer made a number of post-mortems in pigeons suffering from this disease without finding any gross tissue changes.

This disease needs further investigation but the fact that an organism has been found in the duodenum in large numbers where it multiplies and apparently produces by-products that are absorbed, and which interfere with the normal metabolism of the body, is of sufficient interest to call attention to the preliminary findings herein mentioned. It is not unlikely that if the present hypothesis concerning the nature of this disease is verified that a number of disorders now attributed to general causes may be traced to some form of intestinal infection.

REFERENCES.

I. DAwsox. Asthenia (going light) in fowls. Annual Report of the Bureau of Animal Industry U.S. Department of Agriculture, ISgS. p. 329 . 
CHAPTER IV.

DISEASES CAUSED BY BACTERIA-GENUS BACILLUS.

HOG CHOLERA.

Synonyms.* Swine fever; Pneumo-enteritis; Pig typhoid ; Svinpest.

S I3I. Characterization. The distinguishing features of this disease are a continuous fever, ulceration of the intestines, and more or less discoloration of the skin, especially over the ventral surface.

I32. History. The earliest outbreak in this country of which there is knowledge of a disease supposed to be hog cholera occurred in the state of Ohio in 1833 . It is presumed that it was brought from Europe with sone of the animals imported from there for breeding purposes. After beingintroduced, it spread at first slowly, but later with increasing rapidity along the lines of commerce, until it invaded every part of this country where swine raising had become an industry. The disease was investigated and very carefully described by Dr. C. Sutton of Aurora, Ind., from 1850 to I858. In I86 I, Dr. Edwin M. Snow, of Providence, R. I., contributed an important paper on this disease to the U.S. Department of Agriculture. In i 875, Dr. James Law, of Ithaca, N. Y., furnished to the same Department a paper

*This disease is known popularly by a large number of names and in some works on swine diseases many of them are employed. The more common of these are, enteric fever, typhus carbuncular fever, carbuncular gastro-enteritis, carbuncular typhus, pig distemper, blue sickness, blue disease, purples, red soldier, anthrax fever, scarlatina, measles, diphtheria, and erysipelas. Many of the terms appear to refer to sonie one or more of the observed symptoms or lesions. 
setting forth the symptoms and morbid anatomy of this disease. He believed it to be contagious although the specific organism had not been found. The U.S. Commissioner of Agriculture appointed, in I 878, nine wen for a period of two months each to investigate the disease in various localities. In their reports the symptoms and norbid anatomy formerly described were confirmed and two additional features set forth. Law showed that it was transmissible by inoculation to other aninals (rabbits, rats and sheep) and Dr. Detmers described a microorganisn which he called Bacillus suis and which he beliered to be the specific cause of the trouble. Later, Detmers described his organism as a micrococcus. The transwission of the malady to rats and sheep was not confirmed by subsequent tests. The work of investigation was continued under the direction of the Commissioner of Agriculture and finally, in 1885 , the specific organism was discovered by Salmon and Smith, who described its essential cliaracters and properties. It was called Bacterium of swine plague. Since that time the disease has been under investigation and the Bureau of Animal Industry has during the last few years been actively engaged in the effort to produce a specific, therapeutic serum.

In I 886 , Dr. Theobald Smith discovered another bacterial disease among swine. It was found to be similar to the German Schieineseuche, both in its morbid anatomy and in the morphology and properties of its specific organism (see $\$ 47$ ). In naming this disease the Bureau of Animal Industry called it, on account of its similarity to the German Schweineseuche, swine plague and its organism the bacillus of swine plague, and changed the name of the disease described in 1885 to hog cholera and its organism to the bacterium* of hog cholera. The changing of the name of the first disease described from swine plague to hog cholera lias been the cause of some criticism and it has been credited with the responsibility of creating confusion. It has, perhaps, led hasty readers to a misinterpretation of these diseases and their relation to those des-

*In 1888 the genus Bacterium was changed to Bacillus and this organism is spoken of since that time as the hog-cholera bacillus. 
cribed in other lands under different titles. While the nantes assigned may not have been especially happy ones, the transfer of swine plague from the intestinal to the lung disease must be considered as a fortunate occurrence and one which tended to simplify and not to confuse.

Billings, of the Nebraska State Experiment Station, opposed this nomenclature. He not only refused to accept the cliange and continued to write about hog cholera under the title of swine plague, but he denied the existence of the swine plague, as described in the reports of the Bureau of Animal Industry for 1886 and subsequently, as an independent disease. The wide dissemination of his publications on this subject has unquestionably been responsible for much of the haziness concerning the distinguishing features of these maladies.

In r893, Drs. Welch and Clenents read a paper before the International Veterinary Congress in which they gave a very clear history of the nomenclature of these diseases and in which they adhered to the one of the Bureau of Aninal Industry.

S 133. Geographical distribution. Hog cholera is widely disseninated throughout the central part of the United States. It exists, however, to a certain degree in every state in the Union and in Canada. It has long been known in Great Britain. It prevails to a greater or less extent on the European continent. The confusion which has arisen in the use of the terms swine plague and hog cholera renders it difficult to determine from the brief description in a number of reports the nature of the malady in question.

I34. Etiology. The specific disease, here described as hog cholera, is caused by Bacillus cholerae suis. A brief description of its morphology, physiological properties and pathogenesis are appended.

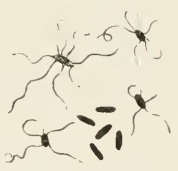

FIG. 39. Bacillus of hog cholera both with and without flagella.

135. A brief description of the bacillus of hog cholera. 
Morphology.-A rod-shaped organism varying in size according to the medium in which it has developed. From agar cultures it is from I. 2 to $1.8 \mu$ long and from .5 to $.8 /$ broad. The ends are rounded. Spores have not been observed. It is actively motile and a variable number but usually from 3 to 5 flagella have been demonstrated. The length of the flagella also varies. The average seems to be about $7 \mu-$ although filaments 55 microus with an average length of 35 to $40 \mu$ are reported by Ferrier. It stains readily with the aniline dyes. Preparations nade from cultures usually stain uniformly; while in the preparations made from the tissue of inoculated animals there is frequently exhibited a light center with a deeply stained periphery.

Cultural characters and biochemic properties. -This bacilius is grown readily on all of the ordinary media used in bacteriological work at a temperature of 30 to $38^{\circ} \mathrm{C}$. It is aërobic and facultative anaërobic.

Agar.-On the surface of inclined agar after 24 hours at a temperature of $37^{\circ} \mathrm{C}$. a grayish, glistening nonviscid growth appears. When isolated the colonies are nearly round, convex, 0.5 to $2.0 \mathrm{~mm}$. in diameter. The edges are sharply defined and even. In stab cultures a gravish growth develops along the needle track with a more vigorous growth on the surface about the needle puncture. The growth reaches its maximum in about 48 hours.

Gelatin.-In this medium the growth is moderately feeble, the colonies appearing as grayish dots. When magnified they are finely granular and of a yellowish tint. The quantity and form of growth depends considerably upon the reaction of the gelatin. If decidedly alkaline there is often a tendency for the growth to spread. There is no softening or liquefaction of the medium.

Potato. - The growth on potato takes the form of a very thin, glistening layer. It is usually of a faintly yellowish color but this is subject to variation on different potatoes. If the reaction is strongly acid no growth appears.

Bouillon.-In alkaline bouillon it imparts in 24 hours a uniformly cloudy appearance to the liquid. Ordinarily there is no membrane on the surface. After some days' standing the growth begins to settle, forming a grayish, friable sediment. If the bouillon contains muscle sugar the reaction will be changed to acid, in from 24 to 48 hours, due to the fermentation of the sugar. Later, however, the liquid will become strongly alkaline, unless there was too much muscle sugar present. In acid bouillon the growth is less vigorous. It grows better in a bouillon containing peptone than in a simple beef broth.

Milk, - When the milk is acid in the beginning it gradually becomes alkaline. There is no precipitation or coagulation of the casein. After standing for from two to three weeks in an incubator a gradually developing opalesence of the milk can be observed. Later it beconies clear, then light brownish in color. If allowed to stand longer in the incubator the volume of the culture shrinks by evaporation and the 
opalescent liquid becomes quite thick and dark-colored but not viscid. When the opalesence appears the milk is strongly alkaline. The process seems to be a form of saponification of the fat globules due to the presence of the alkali produced by the bacteria

Indol.-In Dunham's solution the growth is quite feeble. Ordinarily no indol reaction is obtained although it has been observed in a few cultures obtained from different epizoötics.

Gas production.-In peptonized bouillon containing I per cent. dextrose, gas appears within 24 hours and continues to form for from three to five days. During the first day from one-fourth to one-half of the total quantity is produced. By the end of the second day the gas formation is nearly at an end. The total amount which collects in the closed branch of the fermentation tube is equivalent to about one-half of the capacity of this branch. The gas set free is composed of $\mathrm{CO}_{2}$ and an explosive gas which consists largely of $\mathrm{H}$. The ratio of $\mathrm{CO}_{2}$ to $\mathrm{H}$ in the fermentation tube is approximately as $1: 2$. The reaction of the liquid becomes strongly acid, which condition checks the multiplication of the bacteria.

Gas is not produced in bouillon containing lactose or saccharose. These sugars are not fermented. Alkaline cultures containing them become more strongly alkaline as the growth continues.

Thermal reactions. - This organism grows very feebly at a temperature of $20^{\circ} \mathrm{C}$. It will not thrive at a temperature above $43^{\circ} \mathrm{C}$. It is destroyed when exposed to moist heat at $55^{\circ} \mathrm{C}$. for to minutes.

Disinfectants. - This organism is destroyed after an exposure for Io minutes or less in the following solutions:

Carbolic acid, I per cent. Hydrochloric acid, I-5 of I per cent. Sulphuric acid, 1-20 of I per cent. Sulphate of copper, I-4 of I per cent. Formalin, I to 2,ooo. Trikresol, I-2 of I per cent.

Lime is also a good disinfectant when used in preparations containing about 1 per cent $\mathrm{CaO}$.

Drying.-This bacillus resists drying for a variable length of time, according to the amount of protection it has. In a drop of a bouillon culture dried on a cover-glass and kept under bell jars, the vitality is retained for from 5 to $S$ days. In bits of animal tissue containing the bacilli, the vitality is retained for from 20 to 40 days, according to the quantity of tissue taken.

Pathogenesis.-Subcutaneous injections of from I to 3 c. c. rarely produce fatal results in swine. An intravenous inoculation of 5 c. c. usually produces a septicaemia. With smaller doses the "button ulcers," characteristic of hog cholera, have been produced (Welch). By feeding pigs with pure bouillon cultures the intestinal lesions, typical of hog cholera, have also been obtained (Smith).

Rabbits inoculated subcutaneously with 0.1 c. c. of a bouillon culture die in from 5 to $S$ days. The essential lesions consist of necrotic 
foci in the liver and a very much enlarged and dark-colored spleen. Guinea-pigs are affected similarly to rabbits, but death does not usually occur until from 7 to 12 days. Pure cultures of the bacillus can be obtained from the blood, liver or spleen of the inoculated animals.

While the above description applies to the form most frequently encountered, varieties are not uncommon. In IS94 Smith called attention to several varieties of this species. It is interesting to add, tirat Reed and Carroll have found the bacillus isolated by Sanarelli, and tliought by him to be the cause of yellow fever, to belong to this group of bacteria.

s 136 . Symptoms. The symptoms of hog cholera are by no means constant. The best informed writers on the subject agree that hog cholera can not, with certain exceptions, be positively diagnosed from the symptoms. Animals suffering from various intestinal troubles frequently exhibit symptoms which very closely resemble those of this disease.

There are two recognizable forms, namely, the acute and the chronic or mild form. In the acute disease, the animals die very suddenly after a few hours or at most a few days' sickness. In the other form, the disease runs a longer course. There is usually a rise of temperature of from $\mathrm{I}$ to $3^{\circ} \mathrm{F}$.

The sick animals act dumpish, spiritless and lie quietly in a corner or huddle together usually concealing the head in the litter. They refuse to move when disturbed and are more or less oblivious to their suffering. The appetite varies. In acute cases the animals may eat quite heartily up to within a few hours before death. In more chronic forms they eat fairly well until the end. There may or may not be diarrhoea. Frequently the bowels are costive. It is quite common in these cases to have an active diarrhoea during the last few days. The color of the discharge depends largely on the food. Vomiting rarely occurs. The changes in the respiration and the pulse are difficult to determine. There is rarely any cough. Usually there is considerable reddening of the skin on the nose, ears, abdomen and on the inside of the thighs and pubic region. Occasionally this reddening is very marked. The redness is diffuse and nore intense as death approaches. In some cases there is a discharge from the eyes. In the chronic form the animal becomes emaciated. These symptoms vary to such an extent that it is sometimes necessary to make a post-mortem 
examination and even then the diagnosis must often be delayed until the results of a bacteriological examination have been obtained. It not infrequently happens that swine suffering from hog cholera are attacked with swine plague, the two diseases co-existing in the same animal.

S 137. Morbid anatomy. (a) The acute type. This might with equal propriety be called the hemorrhagic or septicaemia type, inasmuch as the chief and perhaps the only obvious changes are hemorrhagic in nature. They are more conspicuous when an aninal is examined immediately after death. The spleen is variably enlarged, soft, and gorged with blood. Sometimes it is twice as long as the normal spleen and the other dimensions being proportionately increased it may extend across the median line to the right side. Next to the spleen, the lymphatic glands and serous membranes are most severely involved. The cortex of the glands appears on section as a hemorrhagic line or band, according to the amount of extravasated blood, or the entire gland may be infiltrated with it. Among the glands most commonly hemorrhagic are those of the meso-colon, those at the root of the lungs, and on the posterior thoracic aorta. Besides these, the retro-peritoneal and the gastric glands may be involved. Nore rarely the mesenteric glands show slight blood extravasations. Hemorrhages are also quite frequent beneath the serous surfaces of the abdomen and thorax. They are most abundant as petechiae and larger patches under the nucous membrane of the large and small iutestines. They are occasionally found under the peritoneum near the kidneys, the diaphragm and the costal pleura as extravasations nearly an inch in diameter.

The lungs, in a small percentage of cases, show subpleural ecchymoses in large numbers and on section small hemorrhagic foci are observed throughout the lung tissue. In a few cases severe hemorrhages involving one or more lobes have been observed. The kidneys are occasionly the seat of extensive hemorrhagic changes. The glomeruli appear as blood red points ; larger extravasations occur in the medullary substance and blood may collect around the apices of the papillae. The subcutaneous tissue over the ventral surface of the body may be 


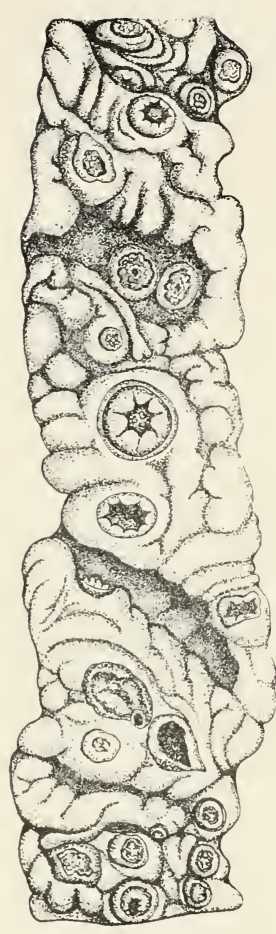

dotted with petechiae and occasionally collections of blood (hematomata) are found in the superficial muscular tissue. The brain and spinal cord have not been generally examined. In one case, petechiae were observed on the cerebellum.

The digestive tract usually is the seat of extensive lesions. The fundus of the stomach is as a rule deeply reddened; there may be more or less hemorrlage on the surface, giving rise to patches or larger areas of blood clots. In some cases the small intestine has subnucous ecchynoses throughout its entire length. In the large intestine these may be so numerous as to give the nembrane a dark red appearance. The intestinal contents are now and then found incased in a layer of blood clot.

(b) The chronic form is perhaps the most common, at least in those epizoötics which have been reported. The acute hemorrhagic cases usually die in the beginning of an outbreak and are apt to be overlooked. Following these are the more protracted ones. In these animals the disease may be

Fig. 40. Ulcers in the limited in its manifestations to the intestine of a pig dead of large intestine although the other orhog cholera.

gans are not exempt from degenerative changes. These are due in part to the impairment of the functions of the large intestine, consequent fermentations and the absorption of the poisonous products elaborated by the specific bacilli in the spleen and other organs.

The lesions of the large intestines are necrotic and ulcerative in character. The ulcers may be isolated and appear as 
circular, slightly projecting masses, stained yellowish or blackish or both in alternate rings, or they may be slightly depressed and somewhat ragged in outline. When the superficial slough is scraped away many ulcers show a gray ish or white base. A vertical section reveals a rather firm neoplastic growth, extending usually to the inner muscular coat. When sections of such an ulcer are stained with aniline dyes and examined under the microscope, the submucous tissue is very much thickened, infiltrated with round cells and containing a large number of dilated vessels. Resting upon this thickened submucosa, is a line of very deeply stained amorphous matter and upon this is situated the necrotic mass which fails to retain the coloring matter and which is permeated by an immense number of bacteria of various kinds. Frequently the eggs of trichocephalus are imbedded in the slough.

The extent of the submu-

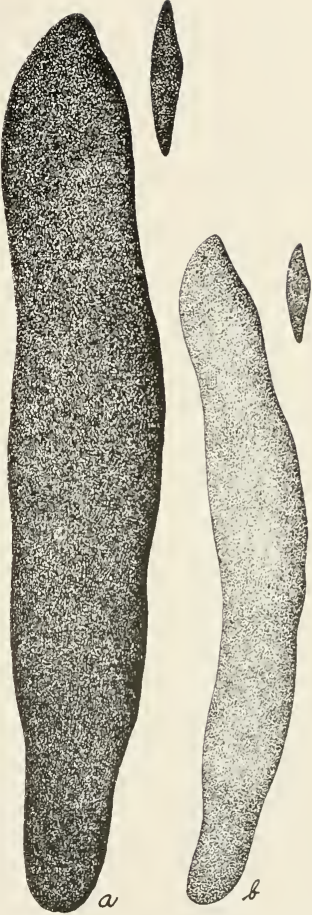

Fig. 41. Spleens of pigs of the same age; $(a)$ dead from hog cholera; (b) normal, killed in heallh).

ulcers it contains many newly-formed capillaries and evidences of the formation of connective tissue are present. The capillaries may extend to the very edge of the border where the slough begins. The latter may have been partly shed, leaving a smooth line bounding the cicatricial tissue. The submucous infiltration gradually disappears toward the periphery of the ulcer 
and slightly outside of the ulcer no inflammation of the membrane exists. Giant cells have been observed in the intertubular tissues at the edge of the ulcer. The depth to which the infiltration extends is not always limited to the submucosa ; it may extend into the muscular coats and cause inflammatory thickening and inflammation and the formation of new vessels in the subjacent serosa.

In some cases the necrosis. instead of appearing in circumscribed ulcers from one-sixteenth to one-half inch or more across, involves the whole surface of the mucous membrane, giving it the appearance of a so-called diphtheritic membrane. In such cases the walls of the intestine are very much thickened and so friable as to be easily torn with the forceps in handling them. Such necroses are rare in epizöotic cases, but it frequently appears in animals which have been fed with pure cultures of hog cholera bacilli.

The distribution of the ulcers varies but slightly. They appear most frequently in the caecum and on the ileo-caecal valve, as well as in the upper half of the colon. The lower half is implicated in severe cases only and then less extensively. The rectum is rarely ulcerated. The lower portion of the ileum is ulcerated in a small percentage of animals, especially when they have been fed with hog cholera viscera or cultures. The stomach is occasionally the seat of slight ulceration. The lymphatic glands of the affected intestine are usually much enlarged, pale, tough and whitish on section. The spleen is rarely enlarged; the liver shows degenerative changes. The heart and lungs are usually normal. The broncho-pneumonia frequently found in young pigs in the winter months must be ascribed primarily to exposure rather than to the presence of hog cholera.

In some outbreaks the acute and the chronic types of the disease are not so clearly separated as given in the foregoing pages. Frequently recent hemorrhagic lesions seen to be associated with cases presenting extensive ulcerations, which certainly are much older than the extravasations. It may be that the latter are the result of a secondary invasion of the hog-cholera virus, either from the ulcers in the intestine or 
from without. To illustrate more fully the differences in the lesions of the two forms of the disease, the published postmortem notes of two cases are appended.

(I) Acute form. Female, two years old, weight about 250 pounds. She had been known to be sick but a few hours. The examination was made two hours after death. A littte blood was oozing from the nostrils. The skin was not discolored. Upon section the flesh was normal in appearance. The liver was deeply reddened due to engorgement of the blood vessels. Blood flowed freely upon section. The spleen was slightly enlarged and dark colored. The kidneys were hyperaemic, especially the medullary portion. In the pelvis of the right kidney there was a large blood clot. The mucous membrane of the intestines was normal with the exception of several irregular areas of hyperaemia. In the fundus of the stomach was a large, dark blood-clot. No ulcers. The mesenteric glauds were enlarged and darker than normal. In a few cases the cortex was hemorrhagic. The right lung was in a state of hyperaemia. The heart contained very little liquid blood.

Bacteriological examination.-A few bacteria were found in stained cover-glass preparations from the spleen and liver. Tubes of slant agar were inoculated with bits of the tissue from the hyperaemic lung, liver, spleen and kidneys. These tubes developed cultures of the hogcholera bacillus. A few of them were pure cultures; the others contained, in addition to the hog-cholera organism, a quite large bacillus. (Report N. Y. State Com. Agric. I $88_{7}$ )

(2) Chronic form. Small female, weight about 5o lbs. Considerable reddening of the skin over the ventral aspect of the body and limbs; especially marked along thé median line. Superficial inguinals enlarged, of a mottled, pale and deep red on section. Spleen very large, 12 inches long, 2 inches broad, and five-eighths to three fourths inches thick at the liilus; gorged with blood, friable. A small number of punctiform hemorrhages in cortical portion of the kidneys. Glands of mesentery and colon enlarged and congested. Deep reddening of several square inches of mucosa in fundus of stomach. Large intestine contains a semiliquid mass chiefly earth. Four large ulcers in the caecum, one of them at least one inch across, covered by a yellowish slough; the peritoneum covering it is thickened and inflamed. In upper colon there is considerable necrosis, involving the epithelium in patches. Lungs normal, excepting the right ventral lobe, which is solid. Bronchi and air cells of this lobe completely occluded by plugs; surface bright red, mottled with yellowish points - the ultimate air cells filled with the cellular exudate. Subpleural ecchymoses over both lungs. From the spleen a liquid and a gelatin culture contained only hog cholera bacteria. They were very numerous in cover-glass preparations from this organ.

A rabbit inoculated from the consolidated lung tissue died on the 
seventh day. At the point of inoculation a pasty mass extends to abdomen, only subcutis involved. Spleen engorged. Single acini in the liver are completely necrosed, yellowish white. In both organs, hog cholera bacteria. Cultures from spleen pure. (Report on hog cholera, I859.)

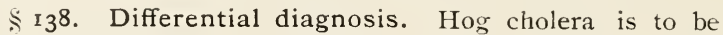
differentiated from a great variety of dietary disorders and poisoning from alkalies and possibly from other chemicals which may get into their food. Powdered soap has been found to produce, when given in sufficient quantities, a series of symptons quite similar to those of hog cholera. In addition to the many as yet etiologically undetermined disorders often giving a high mortality and popularly called hog cholera, infectious pneumonia or swine plague and tuberculosis are to be distinguisked from it.

It sometimes happens that swine when kept under good hygienic conditions suffer from disorders which in their symptoms resemble hog cholera, but anatomically the lesions are varied and irregular. A few such enzoötics have been studied. A few have been described. In one instance $B$. coli communis seemed to stand in a casual relation to the trouble. Recently the writer has studied two similar enzoötics where several animals died and where the lesions were very few and exceedingly varied. In some of them there were healing, intestinal ulcers and the bacillus isolated belonged to the paracolon group. It may be supposed that possibly many of these mild cases (enzoötics) are modified hog cholera. At present, however, such a conclusion does not seem to be fully justified.

The dietary disorders are excluded from the history, the irregularity of the lesions and the failure to find the specific organism of hog cholera in the tissues of the dead animals. An important feature is the fact that the trouble does not extend beyond the herd or herds first attacked or animals fed and kept under like conditions.

In differentiating hog cholera from swine plague much depends upon the specific bacteria. While in typical chronic cases. the intestinal lesions in hog cholera and the lung affections in swine plagne are sufficient, in many cases and outbreaks the variations of the lesions are such that diagnosis must de- 
pend upon the bacteriological findings. The essential differences between the two species of bacteria are brought out in the comparison appendied.

\section{Bacillus of hog cholera.}

1. Rod-shaped organism with ends rounded, 1.2 to $2.0 / 1$ in length, 0.5 to $0.8:$ in width. The size varies according to the stage of growth and division and the culture media.

2. From cultures it stains entirely. In tissues it usually stains around the periphery leaving a light centre.

3. Actively motile in liquids.

4. From 3 to 9 flagella are denonstrable.

5. Vigorous growth in alkaline nutrient liquids. Less vigorous if liquids are acid in reaction.

6. Moderate growth on potato. (Varies according to reaction.)

7. Distinct growth on gelatin.

8 Saponifies milk in from 3 to + weeks.

9. Ferments dextrose with the formation of acids and gas.

ro. Does not ferment lactose. Bouillon containing it becomes strongly alkaline. No gas.

II. Does not ferment saccharose Bouillon containing it becomes strongly alkaline No gas.

12. Destroyed by moist heat at $5^{8^{\circ}} \mathrm{C}$. in 15 minutes.

13. Dies in water in from 2 to 4 months.

14. It dies in the soil in from 2 to 3 months.

I5. Rabbits injected subcutaneously with o.I c.c. of a bouillon culture of a virulent bacillus will die in from 5 to 7 days. Enlarged spleen, necrotic foci in liver.

\section{Bacterium of swine plague.}

I. Enlongated oval organism 0.8 to $1.5 \mu$ in length, 0.6 to o $8 \mu$ in thickness. The size varies according to the stage of growth and division and the culture media.

2. From old cultures it usually stains entirely. When in process of division as found in the organs of freshly dead rabbits the extremities stain leaving an unstained central band, "polar stain."

3. Not motile in liquids.

4. No flagella have been found.

5. Growth moderate or feeble in alkaline nutrient liquids. No growth if liquids are acid.

6. No growth on potato.

i. Feeble or no growth on gelatin.

S. Produces no apparent change in milk.

9. Ferments dextrose with the formation of acids but no gas.

Io. Does not ferment lactose. No. gas.

II. Ferments saccharose with the formation of acids. No. gas.

12. Destroyed by moist heat at $55^{\circ} \mathrm{C}$. in 7 minutes.

13. Dies in water in from to to 15 days.

I4. It dies in the soil in from 4 to 6 days.

I5. Rabbits injected subcutaneously with o.or c.c. of a bouillon culture of a virulent bacillus will die in from 16 to 20 hours. Septicæmia. 
16. Rabbits inoculated with culture of an attenuated variety live from Io to 20 days or recover. The lesions are enlarged spleen, and infiltration of the follicles in Peyer's patches.

17. In guinea-pigs the lesions are practically the same as in rabbits. Death occurs in from 7 to $\mathrm{I} 2$ days.

18. Pigs are not usually affected by subcutaneous injection of small quantities of culture. If the pigs are killed within I to 3 weeks the bacilli are found in the local lesion and certain of the lymphatic glands. Fatal results are reported in a few cases by these injections.

I9. Feeding cultures to pigs which have fasted for 24 hours produces extensive intestinal lesions with fatal results.

20. Intravenous inoculation into pigs causes either an acute septicæmia or a chronic form of the disease in which are produced quite typical round, firm, elevated ulcers.
16. Rabbits inoculated witl a culture of an attenuated variety will live from 4 to Io days. The lesions are local infiltration of pus cells with pleuritis, pericarditis or peritonitis.

17. Guinea-pigs are slightly less susceptible than rabbits. There is more local reaction. Death occurs in from I to 4 days.

IS. Pigs are not usually affected by the subcutaneous injection of small quantities of culture. The bacilli are not found except in the local lesion. In a few cases fatal results are reported.

19. Feeding cultures to pigs usually produces no effect.

20. Intravenous inoculation into pigs usually produces a septic form of the disease which kills in from 1 to 2 days. Inoculation into the lungs causes pleuritis, usually accompanied by pneumonia.

539. Prevention. As hog cholera is caused by a specific organism the first fact to be determined is to find the channel or means by which it can be carried from an infected to a non-infected herd. The thorough investigations which have been made in the United States Bureau of Animal Industry have shed much light upon this subject. The observations of more recent years have confirmed the conclusion reached in the earlier reports of the Bureau concerning the means of spreading this disease. With these results, the pointing out of the ways by which the virus may be disseminated and the methods necessary for checking its spread is no longer questionable and there is a certainty that it can be kept away from individual lerds even in the midst of wide spread 
epizoötics. A few of the common means of its dissemination are worthy of note.

I. The virus of hog cholera is frequently introduced into a non-infected locality by the purchase of animals, usually for breeding purposes, from herds in which this disease exists or has existed within the preceding few months. These animals are usually placed anong the home raised swine without quarantine thits affording every possible facility for starting up a new outbreak. The reason for this is clear.

The bacteria can be carried in the dirt on the animals or, as is most usually the case, the pigs may have been but recently infected and being transferred during the period of incubation they develop the disease later. It not infrequently happens that the purchased animals are actually suffering from a chronic form of the disease, to which they eventually succumb but meantime infecting others. In purchasing swine, therefore, it is of the greatest importance that the history of the herd should show that it had heen free from infectious diseases for at least one year. In addition to this, newly purchased swine should not be placed immediately after shipment in the pens with the home stock, but they should be kept in a separate enclosure until all danger of the disease has passed.

2. Swine are often shipped in crates, boxes or in open cars in which logs affected with $\log$ cholera have previously been confined. The history of hog cholera contains many illustrations of this method of contracting the disease.

3. The bacilli of hog cholera live for a considerable time in water. On this account the bacteria from outbreaks which start at or near the source of a creek or small river may be carried in the current and infect animals which wallow in the stream many miles below. By keeping swine in a small enclosure away from infected streams and fields the disease is often prevented.

4. The bacilli of hog cholera can be carried in the dirt which adheres to one's shoes or to farming utensils. It not infrequently happens that the virus of this disease is carried from farm to farm on the tools taken from an infected place. 
5. The virus may be carried by buzzards, crows and other birds. There is no positive proof that the virus has been disseminated in this way although there is much evidence to support such a theory, particularly in the South. Several outbreaks have been attributed to this method of introducing the virus. The hypothesis emphasizes the necessity for promptly disposing of the dead animals instead of leaving them as prey for scavengers. If they cannot be burned it is best to cover the bodies with a liberal amount of lime and bury them.

\$ 140. Specific treatment. A large number of investigations have been made to find a protective vaccine method for this disease and also to find a specific serum treatment. Thus far satisfactory experimental results have not been obtained. In a number of instances where the practical applications of the "sernm treatment" has been made, most satisfactory results followed, but the reports fail to give evidence of an accurate diagnosis of the disease treated. In these cases, the better management of the animals, in addition to the serum, would suffice to check the disease if the trouble was of a dietary wature. There is need for additional investigation along these lines.

\section{REFERENCES.}

I. Bitrings. Bulletins Neb. Agric. Expt. Station 1888. Alsomany special publications and contributions to various veterinary journals.

2. DAwSON Theserum diagnosis of hog cholera. New York Med. lour. Feb. 20. 1897 .

3. DE SchwerNitz. The production of inmunity in guinea pigs from lig choie: by the use of blood serum: from irivunized animals. Annual Repart of the Bureau of Animal Industry, U. S. Dept. of Agriculture. $1 S g S$.

4. PETERs. Serum tierapy in hog cholera. Bulletin No. 7 . Uniz. of Neb. Agri. Exper. Station. 1897.

5. SALmox AND Surru. Annual Reports of the Bureau of Animal Industry. $1885-1895$.

6. SAtmon. Special Report on ho cholera, its History, Nature and Treatment. U.S. Bureau of Animal Industry. $18 S 9$.

7. Smith. Zur Kerntniss des Hog cholera Bacillus. Antralbiatt fur Bakter. u. Parasitenkunde. Bd. IX. (IS91) S. 253.

8. Surth. Hoz cholera group of bacteria. Bulletin No. $6 U$.S. Bureau of Animal Industry. 1894. p. 9.

9. SmITH AND MOORE. Experiments on the production of inmu. nity in rabbits and guinea pigs with reference lo tog cholera and swine plague bacteria. Ibid. p. 41 . 
10. REED AND CARROI,L. Bacillus icteroi les and Bacillus cholerae suis. A prelininary note. The Medical News. Apr. 29, I\$99

II. WELCH Report of investigations concerning the causation of hog cholera. Johns Hopkins Hospital Bulletin. No. I. 1889.

I2. WELCH AND CLEMENTS. Remarks on hog cholera and swine plague. First International Congress of America held in Chicago, I11., October, IS93.

\section{TETANUS.}

$\$$ 141. Characterization. Tetanus is an infectious disease (toxaemia) in which specific organism is localized at the place of inoculation. It is characterized by spasmodic contraction of the muscles, referable to the nervous system and by the absence of obvious tissue changes. It is the result of a specific wound infection. All mammalia including man are susceptible. It occurs most frequently in horses, asses and mules; next to them in the smaller ruminants such as the sheep and goat; it appears least often in the dog. It is reported to occur rarely in birds and fowls are supposed to be immune. The human species is very susceptible.

S 142. History. Tetanus is one of the diseases that was recognized and described before the Christian era. It was not clearly differentiated until the discovery of its specific cause in 1884 .

\$ 143. Geographical distribution. Tetanus is reported to be more prevalent in the hot climates than in the teniperate ones, while in the very cold latitudes it is rarely if ever encountered. It is more frequently met with in some districts than in others. Although very common in certain localities, it is, on the whole, a somewhat rare disease. There seems to be no statistics by which its frequency can be determined in this country, but in certain of the European armies this has been noted. In the Prussian army, it is reported to occur once in a thousand cases of sickness among horses. At Württemberg, Hering reports it once in 3000 cases of disease among the horses in the cavalry. It has also been noted that in some veterinary hospitals it does not occur for long periods, while at other times several cases may appear in rapid succession. It is, however, a wide spread disease. 
\$ 144. Etiology. Tetanus is caused by a slender bacillus 2 to $5 \mu$ in length. It forms spores which are at the end of the organism giving it somewhat the appearance of a pin. On account of this it has been designated the "pin bacillus." It is anaërobic. This organism was first observed by Nicolaier in 1885 , although Carle and

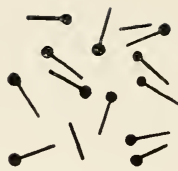

FIg. 42. Bacillus tetani. Rattone showed in I 884 , that this disease could be transmitted from man to animals by inoculation with the pus from the local lesion. In I889, Kitasato isolated the bacillus and studied it in pure culture. This bacillus stains readily with the aniline dyes, especially with carbol-fuchsin, It takes the Gram stain. It grows well in nutrient gelatin, agar or bouillon and on blood serum at the temperature of the body and in an atmosphere of hydrogen or in the absence of air as in deep agar cultures. The addition of a little grape sugar facilitates its growth. It has the distinction of producing the most powerful (poisonous?) toxin of any known bacteria, 0.23 of a milligram being estimated as a fatal dose for a man of 175 pounds weight.

The fact that this bacillus is an anaërobe renders its cultivation of little practicai value in diagnosing the disease. Although it is not distributed in the body, it can usually be found in cover-glass preparations made from the local lesion and stained with carbol fuchsin.

Bacillus tetani is found in the soil. It lias been found in hay dust, in the mortar of old masonry, in the dust in rooms, barracks and hospitals and in the arrow poison of certain savages in the New Hebrides. They obtain it by smearing their arrow heads with mud from crab holes in the swamp. It is reported that certain savages in Africa destroy their enemies by putting bits of broken glass mixed with certain soils in their shoes. The cause of death is tetanus. Mould rich in horse manure seems to be the most favorable abode for it. It has been stated that it exists in all soils. There are good reasons for believing that this is an over estimate of the wideness of its distribution. It certainly is more numerous in some localities than in others. 
The tetanus bacillus is very resistant, especially in its spore form, to destructive agents such as drying and the ordinary disinfectants. Kitasato found that a 5 per cent solution of carbolic acid applied for ten hours failed to kill the spores. Tizzoni and Cattani found that mineral and organic acids produced no effect upon the dried spores. Behring found that iodine trichloride possesses a strong antiseptic effect upon them. They are not affected by the gastric fluids. It has been noted by Kitt that the dried spores in pus have retained their virulence for sixteen months. They are destroyed when subjected to a temperature of $100^{\circ} \mathrm{C}$. in water or steam for ten minutes. The bacilli in the regetative state are readily destroyed by the usual strong disinfectants, such as 5 per cent carbolic acid.

A number of psendo-tetanus bacilli have been described. This renders a careful study of the suspected organism necessary as it is difficult in some cases to determine microscopically $B$. tetani. The guinea pig inoculation affords a ready means of differentiation whenever fresh material is available.

$\$$ 145. Mode of infection. As the bacillns of tetanus is widely distributed in the soil and consequently on articles contaminated with it, the most common modes of infection are punctures scratches, and pricks made by splinters, nails or infected instruments (traumatic tetanus). It may follow slight abrasions of the skin where infected earth comes in contact with the lacerated epidermis. Infection through wounds in the intestinal mucosa do not seem to have been clearly demonstrated. The most usual method seems to be by pricks and nail punctures, in which cases the virus can be carried well into the living tissues and there is little or no bleeding to wash it out.

Infection often occurs in young foals and lambs through the freshly broken umbilical cord (tetanus neonatorum). The symptoms begin to appear in a variable length of time after the infection takes place. The shortest period which seems to be reported is a few hours and the longest is six weeks. In horses the period of incubation is usually from four to twenty days. After inoculation with pure cultures it is from four to five days and in sheep from two to four days. 
In guinea pigs inoculated with infected soil the incubation period is usually not over forty-eight hours and often less than that. Park has found that mice, guinea pigs, rabbits, rats, horses, goats and a few other animals inoculated with pure culture have a period of incubation of from one to three days. In man it varies from one to twenty days. There are, however, a few exceptionally long periods reported. It has been noted by Richter and others that the shorter the period of incubation the more severe the disease, the mortality being over 90 per cent in the first and about 50 per cent when the symptoms were slow in appearing.

$\$$ r46. Symptoms. The first symptoms are often obscure and may be overlooked for several days or they may be ushered in suddenly with violent and extensive tonic spasms. The tetanic spasms usually begin in the muscles of the head and neck, extending from these to the muscles of the throat, trunk and extremities. It often happens that the spasms first appear in the hind quarters and extend forward. There is stiffness of the parts affected. If in the head, the muscles of mastication are first attacked with spasms, while if the hind quarters are first attacked, there are usually spasms of the muscles of the tail. The muscles at the site of inoculation are frequently the first to show spasms and, if the disease is of a mild type, they may be the only ones to exhibit symptoms. Friedberger and Fröhner have grouped the muscles which are attacked with the more obvious effects upon the appearance of the animal. They are as follows, viz.:

(1). The muscles of mastication. The contraction of these muscles is called trismus or "lockjaw." According to the degree of contraction the jaws remain in more or less close contact, rendering prehension or mastication difficult or inpossible.

(2). The other muscles of the head. These are spasmodically contracted in different degrees. Spasms of the muscles of the ears cause the ears to be "pricked" and their tips to be drawn together; of the recti muscles of the eyes, cause the eyes to be retracted in the orbit with protrusion of the nictitating membrane; of the nose, produce dilatation of the nostrils ; 
of the dilator of the upper lip, give an abnormal shape to the opening of the mouth. The muscles of the tongue, of deglutition and of the larynx are also usually attacked by spasms.

(3). The extensor musiles of the neck. Contraction of these muscles cause a stiff, stretched out carriage of the head and "ewe neck." 'The muscles of the neck become hard and tense to the touch.

(4). The extensor muscles of the back. Spasms of these muscles are manifested by an extremely hard condition of the muscles of the back, loins and croup. Several conditions may. arise. Orthotonus in which the neck is stretched out and the back and croup are carried horizontally or, opisthotonus in which the head is raised and drawn back and the vertebral column slightly depressed. This is the most common occurrence. There may be a lateral curvature of the cervical vertebrae which is uncommon and also a convex curvature of the vertebrae which is very rarely observed. The tail, especially in horses, is often raised and occasionally said to be straight with the back.

(5). The muscles of the limbs. The spasms in these muscles make the limbs stiff and cause the animal to assume an attitude in which the fore legs are extended forward and laterally and the hind ones backward and laterally. They are bent at the joints only with difficulty. The contraction of the muscles of the abdomen gives the animal a tucked up appearance and the spasms of the muscles of respiration render breathing difficult.

Besides the spasms the animal shows an increased reflex irritability and heightened sensibility. These manifest themselves in excitement, timidity and intensified muscular contractions if irritated. Sweating is common especially in severe cases. In mild cases it may be absent. There is usually little or no change in the internal temperature. In fatal cases the temperature is usually constantly higl toward the last. The high temperature ( $104^{\circ}$ to $106^{\circ} \mathrm{F}$ ) usually continues for some time after death. Bayer has observed in a horse, 24 hours before death, a temperature of $102^{\circ} \mathrm{F}$; one and a half hours before death, $105^{\circ} \mathrm{F}$; at the moment of death, III ${ }^{\circ} \mathrm{F}$; 
and fifty minutes after death, $I 13^{\circ} \mathrm{F}$. There is frequently no increase in the number of pulse beats until severe exacerbations set in. The frequency of the pulse is much greater in animals which continue recumbent than in those which keep upon their feet. The pulse is often hard and small and the walls of the arteries are spasmodically tense. In many cases however, it is full, soft and easily compressible. There is, as a rule, an increase in the number of respirations, which may become very high if the respiratory muscles are attacked. The number varies according to the excited condition of the animal. The respirations may increase four fold without a corresponding increase in the pulse beat. The breathing may reach from so to $\mathrm{I}$ oo per minute. In character the respirations are shallow on account of the fixed condition of the ribs and the spasms of the muscles which compress the abdomen. There may be cyanosis and catarrh of the nasal mucosa, coughing and in fatal cases symptoms of hyperaemia and oedema of the lungs and often pneumonia (usually aspiration in nature). There is constipation due to lack of peristalsis and the rigid condition of the muscles which compress the abdomen. Micturition becomes less frequent and more difficult. Complete retention of urine is said to occur in some cases. The urine has a high specific gravity and in some cases contains albumen. Some animals can eat readily while others eat, if at all, with great difficulty. They like to play with drink set before them and often try to satisfy their thirst, which seems to increase as the disease advances. In fatal cases the animals seem to be perfectly conscious to the last. They seem to be possessed of a feeling of terror.

s 147. Morbid anatomy. The gross examination of the tissues at post-mortem of animals dead from tetanus is usually negative. It has been pointed out by Goldscheider and Flatau that in experimental animals there are certain characteristic changes in the motor cells of the anterior horns of the spinal cord which in the order of their development depend upon the concentration of the toxin or virulence of the bacteria injected and upon the duration of the disease. The changes are primarily an enlargenent of the nuclei, which at the same time 
become more indistinct ; then follows an enlargement and disintergration of Nissl's cell-grannles with an enlargenent of all of the nerve cells. These investigators also found that where antitoxin has been used it had a distinct retarding influence upon these changes. They found like lesions in the spinal cord of a human subject dead of tetanus. Very similar results have been obtained by Matthes, Westphal, Goebel and others. The lesions point to the anterior horns of the spinal cord as the primary seat of origin of the tetanic contractions. The changes pointed out above are said by Moschowitz to be characteristic of tetanus and constantly found. The motor ganglia cells of the anterior horns of the spinal cord seem at present, therefore, to be the most likely source of the spasms, dne apparently to a specific affinity between those cells and the tetanus toxin. It is possible to explain also the local spasms on this hypothesis as the toxin elaborated by the tetanus bacilli is taken up and carried to the spinal cord by the nerves terminating in the affected region. The experiments of Tizzoni and Cattani suggest the possibility of sucl a theory. There is, however, need for further investigation of this subject.

A considerable number of lesions may be found elsewhere in the body none of which can be considered as characteristic of the disease, but which are secondary to the tonic contractions. The blood owing to lack of oxidation may be dark colored, tarry, of a greasy appearance and tardy in coagulating. There may be numerous ecchymoses and sanious exudates in the subserous and mucous membranes. The lungs may be variously affected according to the extent of the trouble with the respiratory muscles. Thus, congestion, oedema, hemorrhages, pneumonia, emphysema and hypostatic congestions have been described. In the heart there are usually epi- and endocardial hemorrhages. The muscles may contain hemorrhages. The fibers of the muscles may show cloudy swelling, a loss of the transverse striae and changes in the nuclei. The liver may be swollen and abnormally yellow in color. The hepatic cells often show fatty degeneration. The spleen is often swollen; it may be engorged with blood or soft and flabby. The kidneys may or may not show degenerative changes. The blad- 
der is usually distended with urine and its mucous membrane is often sprinkled with ecchymoses. The digestive tract may show areas of congestion and ecchymoses.

The course of the disease varies in different species and in different individuals of the same species. In the horse it may last for two or three days only or it may continue for several weeks. In cattle the course is usually less rapid, but it rarely runs longer than two weeks. In sheep it ustually terminates fatally within a week and often in two or three days.

148. Differential diagnosis. Tetanus, while possessing quite characteristic symptoms, may be mistaken for a number of other affections or specific diseases. Among those which should receive special attention are cerebro-spinal meningitis and rabies (for the symptoms and lesions see those diseases). Rheumatism, eclampsia, catalepsy, convulsions in the newly born and pyæmic polyarthritis in lambs and foals.

The symptoms of tetanus which are perhaps the most diagnostic are (I) the continuous tonic spasms of different groups of muscles, (2) the apparent clearness of mind, (if we may attribute such a quality to animals) and (3) the absence of fever in the beginning of the symptoms. The general attitude of the animal is also of much value. If the infected wound can be found it is often possibie to obtain coverglass preparations in which the tetanus bacilli can be found. Negative results are in this case not to be considered as final for it is practically impossible to make these examinations sufficiently thorough to be sure of the absence of these bacilli, if they are not found. If they are found the diagnosis may be considered as positive.

In poisoning with strychnine, there are symptoms which at first may be more confusing. This form of poisoning usually occurs in dogs where tetanus is rare and again in strychnine poisoning the suddenness of the attack, the rapidity of the course and the increased reflex irritability are valuable diagnostic features. In differentiating tetanus from other affections as those in the newly born, the bacteriological examination of preparations made from the end of the umbilicus may be of much assistance. 
\$ 149. Prevention. Owing to the wide distribution of tetanus bacilli, precautions can consist only of the careful and thorough disinfection of all wounds. With animals at pasture, it is impossible often to know of the wounds until it is too late to apply this measure. In stables where the disease becomes prevalent, the floors and siding should be thoroughly disinfected and special watchfulness exercised to find at the earliest moment any injury by which infection could occur. The practitioner should learn as soon as possible the tetanus infected lands and stables in his commnity and, knowing these, give wise instruction to his clients to take such precautions as are possible. If the present knowledge of this disease is properly availed of, there should be only occasional cases which as yet there seems to be no way to avoid.

s I0. Tetanus antitoxin. It was first pointed out by Behring and Kitasato that aninals could be made immune to tetanus by using cultivations of the tetanus bacilli which had been attenuated with iodine trichloride. The blood serum of such immunized animals has the power to immunize healthy animals against the disease and to render the toxin in animals affected with tetanus inert. The antitoxin is prepared now, however, by injecting horses with the filtrate of bouillon cultures, either alone or with a quantity of antitoxin. After the first dose the animal becomes tolerant to a certain degree so that by repeated and constantly increasing doses complete resistance to the toxin is acquired. When this point is reached the serum usually possesses a strong antitoxin power. As a practical remedy for the disease in animals the recorded results from the use of this antitoxin are somewhat contradictory. In luman practice the results are similar. Moschcowitz has collected 290 cases in man, where it has been used subcutaneously with 173 recoveries and 117 deaths or amortality of 40.33 per cent. In a total of 48 cases where the antitoxin has been injected intracerebrally 23 recovered and 25 died, a mortality of 52.08 percent.

Sone interesting experiments suggested by Krokiewitz directed toward the finding of a specific treatment consist in the injection of an emulsion of brain substance. Prinarily 
this method of treatment is based upon the hypothesis, set up by Goldscheider and Flatau, who, as a result of their research came to the conclusion that "The morphological changes in the nerve cells are the expression of a chemical process, i. e., of the chenical combination of the toxins with the nerve cells. Every nerve cell possesses atom groups which have a certain affinity for the atom groups of the tetanus toxin and are able to combine with them." Wassermann and Takaki substantiated this hypothesis experimentally ; these observers injected into experimentally tetanized animals an emulsion of spinal cord, obtained from a freshly killed animal, to test, if possible, whether the nerve cells of the dead animal also have this affinity for the tetanus toxin, like the nerve cells of the living animal. By this experiment, they have come to the conclusion that every part of the nervous system, particularly the brain of the examined animals including man, has a definite and positive tetanus antitoxic power; and that the injection of normal brain substance into experimentally tetanized animals has the power to save life. Further work in this direction is necessary to fully demonstrate the efficiency of this procedure.

\section{REFERENCES.}

I. BEHRING AND KITASATO. Ueber das Zustandekommen der Diphtherie-Immunität und der Tetanus-Immunität bei Thieren. Deutsche Med. Wochenschrift. Bd. XVI. (IS9o) S. 113.

2. Kitasato. Ueber den Tetanusbacillus. Zeit.f. Hygiene. Bd. VII. (1889) S. 225 .

3. KITASATO. Experimentelle Untersuchungen ïber das Tetanusgift. Zeit.f. Hygiene. Bd. X. (1891) S. 267.

4. Moschcowitz. Tetanus, a study of the nature, excitant, lesions, symptomatology, and treatment of the disease, with a critical summary of the results of serum therapy. Studies from the Department of Pathology of the College of Physicians and Surgeons, Columbia University. Vol. VII. (IS99-I900). (M. gives pathology and antitoxin treatment, summary of cases and full bibliography.)

5. MCFARLAND. Tetanus and vaccination. The Journal of Medical Research. Vol. VII. (I9O2) p. 474. 
Synonyms. Black quarter; black leg; quarter ill ; quarter evil; Charbon symptomatique; Rauschbrand.

$\$$ I5r. Characterization. Black quarter is an acute infectious disease of cattle characterized by the development of an emphysematous swelling of the subcutaneous tissues and muscles. The lesions are usually located upon and ordinarily extend over the greater part of a hind quarter or a shoulder. The disease does not spread from animal to animal by simple contact but the infection takes place apparently from a common source, the soil. The virus seems to exist in the soil in certain localities only. 'Like tetanus, it is a disease following a wound infection.

Symptomatic authrax is a disease of cattle, sheep and goats although the two latter species are rarely attacked. Guinea pigs are very susceptible to inoculation. It is reported that horses, asses and white rats develop local lesions when inoculated subcutaneously with the virus. Other animals seem to be immune. In cattle, it rarely occurs in the very young, under six months, and in adults after the fourth year.

s 152. History. It is supposed that black quarter has existed for hundreds of years although it was not until late in the last century that it was positively differentiated and recognized as a distinct and specific disease. The descriptions given in many of the earlier epizoötics designated as anthrax correspond more exactly with the present knowledge of black quarter than they do of anthrax.

In 1782 , Chabert classified the various antliracoid diseases recognized at that time, into three groups, viz.: (I) anthrax fever or where the disease manifested itself without external swelling; (2) true anthrax, or where the lesions consisted at first of small, hard and very painful swellings followed or accompanied by fever and other general symptoms and (3) symptomatic anthrax or where the swelling was preceded by a rise of temperature, loss of appetite and symptoms of general depression. This classification obtained for nearly a century. Boutrolle, in 1797 , refers to a disease which he called mal de 
cuisse (quarter evil) because it affected the animal in the thigh. Viborg described the disease in Denmark where it has long been known to the laymen and designated by them as raslesyge ("rattle disease"). Its clinical features were very accurately described by Walraff in 1856 . In I 879 , Arloing, Cornevin and Thomas proved the causal relations of a certain microorganism to this disease and thus established its specific nature. A year later, 1880, they described the specific microorganism and demonstrated that the disease could be produced by inoculating susceptible animals with it. Since that time both the organism and the disease itself have been studied by many investigators. In this country, the disease has been under investigation during the last few years by investigators in the Bureau of Aninal Industry.

s53. Geographical distribution. Black quarter exists to some extent in nearly every country in the world. It is reported as occurring in the most northern latitudes in which cattle are kept, as well as in the tropical and temperate zones.

In Europe, it occurs on the pastures on the Alps, where for five months in the year the ground is covered with snow and ice and in America it is quite common in certain northern districts. It has been reported from Asia and from Northern and Southern Africa.

In the United States, it prevails to a greater extent than is generally supposed. The states and territories which, according to the reports of the Bureau of Animal Industry, suffer most from it are Texas, Oklahoma, Kansas, Nebraska, Colorado, North and South Dakota and Indian Territory but a number of the other Western States are badly affected. Many of the states east of the Mississippi river have infected localities, but in a few of the eastern and southern states it seems not to exist. During the last few years infected localities have been found in New York where there has been an annual loss from this disease, but where prior to recent investigations the cause of death had been attributed to poisoning.

\$ I54. Etiology. Black quarter is caused by a motile, rod-shaped microorganism varying from 3 to $5 \mu$ in length and from 0.5 to $1.0 \mu$ in width. The ends are rounded and it 
produces spores. In cultures long involution forms"are often observed. It is anaërobic and consequently will:ast derelop in the presence of air. In suitable culture media under anaërobic conditions or in animal tissues (other than blood) it îmultiplies rapidly with the evolution of gas. The presence of spores renders it very resistant to natural destructive agencies and to the common disinfectants. The bacillus of black quarter or its spores are supposed to gain entrance to the tissues of animals through abrasions of the skin or, possibly, the nucous membranes of the mouth or intestine.

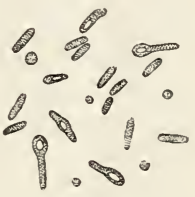

FIG. 43. $\quad B a-$

155. Symptoms. Black quarter runs a rapid course and usually ends fatally in cillus of symptomatic. from one to three days. The first symptoms may be either general or local in character. Arloing, Cornevin and Thomas believe that general symptoms always precede the local manifestations. Nörgaard reports finding cattle with marked local lesions but few animals suffering from general symptons although the latter may have preceded the former and have subsided.

The general symptoms are elevation of temperature, which usually falls to the normal or even suburmal before death, loss of appetite, loss of rumination and pronounced depression. Respiration becomes accelerated, the animal moves with difficulty and lies down frequently. At first the visible mucous membranes are congested and within twelve to fifteen hours they have a dirty leaden or purplish color.

The local symptoms may appear on different parts of the body except below the knee or hock joints and on the tail. They are less frequently found abont the head. They usually appear on the thighs, neck, shoulders and lower region of the chest. The swelling is at first small and painful. It spreads rapidly and may in a few hours attain to a large size when it becomes characterized by a crackling and gurgling sound when the hand is passed over it. On percussion it gives a clear, tympanic sound due to the collection of gas in the affected tissues. At the center of the larger swellings the 
skin becomes dry and parchment like, cool to the touch and painless upon pressure. If lanced, a dark reddish, frothy fluid flows from the wound. It emits a disagreeable odor. In some cases there is but one swelling but usually there are two or more which may become confluent. The lymph glands adjacent to the swellings are much enlarged.

Hun has pointed out the interesting fact that in a very large percentage of cases the swellings appear on the right side. There seems to be no explanation given for this localization. In this country, records are wanting of observations on this point.

Arloing has called attention to a mild form of this disease in which the symptoms are siight debility, loss of appetite and slight local swelling.

5 156. Morbid anatomy. After death the carcasses of animals which have died of this disease soon become distended with gas. 'This is due in part to the fermentation in the digestive tract and in part to the formation of gas in the subcutaneous tissues due to the presence of the specific bacillus. The subcutaneous distension is especially marked in the region of the swellings but it extends for a considerable distance from these foci in the direction of least resistance. The tympanitic condition often causes the two legs on the upper side of the carcass to extend out straight without tonching the ground. A dark, blood-colored, frothy discharge flows from the nostrils and anus. Decomposition takes place very rapidly except in the affected muscles which retain a sweetish-sour odor for a considerable time after other parts of the carcass have become putrid.

The skin covering the swellings is often affected with dry gangrene. The subcutaneous connective tissue is yellow, gelatinous, infiltrated with blood and bubbles of gas which escape if the tumor is incised. The muscles underneath the tumors are of a dirty brown or of a blackish color. At other places they are dark red or dark yellow and, when exposed for some time to the air, they may have a golden lustre. They are brittle, putrid and very rich in fluids. They crackle on palpation. When incisions are made into them, blood of a frothy, 
greasy, tarry appearance and of a sickish, foetid odor issues from them when they are squeezed. The fibres of the muscles show extremely varied degenerative changes. The gases that are present in the muscles are inflammable and burn with a bluish flame on being ignited. They are stated to have but little odor, on which account it is assumed that they consist of carburetted hydrogen. They are also said to contain carbonic acid but no oxygen. An exact chemical analysis of these gases seens not to have been made. The lymph glands near the tumors are enlarged and full of blood. They contain hemorrhages and are infiltrated with a serous fluid. The afferent lymph vessels are sometimes distended with gases, giving them the appearance of strings of beads. Changes similar to those of the external muscles appear in the muscles of the tongue and pharynx when the disease, as may happen in rare cases, is localized in the oral and pharyngeal mucous membranes.

A large amount of blood-red exudate is frequently found in the abdominal carity. In other cases only small quantities of a serous fluid is present. In still others no changes at all appear. The abdominal changes seem to be determined by the swelling of the muscles, $i . e$, whether it has or has not spread to the peritoneum. Yellow gelatinous and hemorrhagic infiltrations are often met with on the omentum, mesentery and in the neighborhood of the kidneys. The mucous membrane of the stomach and small intestine is frequently swollen, congested and infiltrated with hemorrhages, in which case the contents of the intestine are bloody. The liver is hyperemic, but the spleen is usually normal.

In the thoracic cavity, the pleurae in the neighborhood of the swollen parts of the skin and mediastinum are sometimes hemorrhagic. The pleurae may also show large ecchymoses, in which case the thoracic carity contains a sero-sanious exudate. Hemorrhages are sometimes present in the lungs, pericardium, myocardium and under the endocardium. The muscular tissue of the heart is very soft, but the other muscles show only slight changes. The mucous membrane of the 
bronchi are sometimes hyperemic and sprinkled with hemorrhages.

The blood is of a normal color and coagulates readily. The fluids of the muscles have, according to Feser, an acid reaction, and the flesh becomes rapidly putrid. The bacilli of quarter-ill are found only in small numbers if at all in the blood during life, but abundantly a few hours after death. They are numerous in the local lesions.

S 157. Differential diagnosis. Black quarter is to be differentiated from anthrax, the "corn-stalk"' disease, septicaemia hemorrhagica and various forms of poisoning. Usually the localized subcutaneous lesions are sufficient to differentiate black quarter from these other affections. It often happens, however, that post-mortem changes have so modified the carcass before it can be examined that the diagnosis is questionable. In this and all doubtful cases or where only small portions of tissue are sent for examination, it is necessary to resort to a more definite method or methods, viz.: microscopic examination, cultures and animal inoculation.

(1) Nicroscopic examination. Cover-glass preparations made from the fresh tissues will ordinarily exhibit the specific bacteria. They are easily distinguished in case of anthrax and black quarter and often with septicaemia hemorrhagica while in the "corn-stalk" disease and cases of poisoning characteristic bacteria are not found. It is important to eliminate putrifactive bacteria, when the animals have been dead for some hours

(2) Cultures. Ordinary culture media inoculated with the tissues from a case of anthrax will give growths of the anthrax bacteriun, and from cases of septicaemia hemorrhagica that of $B$. boviscpticus, but with the other two aiseases they will remain clear if uncontaminated. The black quarter bacillus will grow on these media only when placed in anaërobic conditions. In case of the "corn stalk" disease the nedia will continue to be clear.

(3) Animal inoculation. In guinea-pigs inoculated in the deeper subcutaneous tissues with pure cultures of symptomatic anthrax bacteria or with bits of tissue from the affected 
area of another animal dead from the disease, death ensues in from one to two days. It is preceded by rise of temperature, loss of appetite and general indisposition. The site of inoculation is swollen and painful and drops of bloody serum may sometimes be seen exuding from it. At autopsy the subcutaneous cellular tissues and underlying muscles present a condition of emphysema and extreme oedema. The oedematous fluid is often blood stained and the muscles are of a blackish or blackish brown color. The lymphatic glands are markedly hyperaemic. The internal viscera present but little alteration visible to the naked eye. In the blood stained serous fluid about the point of inoculation short bacilli are present in large numbers. These often present slight swellings at the middle or near the end. They are not seen as threads but lie singly in the tissues. If the autopsy is made immediately after death, these organisms may not be detected in the internal organs, but if not made until after a few hours, they will be found there also. In recent autopsies only the vegetative forms of the organism may be found, but later (in from twenty to twenty-four hours) spore-bearing rods may be detected. With the "corrs-stalk", disease the inoculated animals will remain well.

$\$$ I58. Prevention. In cliecking the spread of the disease it is very important wherever it is possible to remove the well animals from the infected field and to restrict the sick ones to a small one. The swellings should not be opened and the discharge scattered over the field. The dead animals should be burned if possible, otherwise buried deeply and covered well with line as soon as possible after death. Birds and other aniulals should not be allowed to feed upon the carcasses and the skin should not be removed. Every precaution to restrict the spread of the bacteria of this disease should be taken. It is very desirable to thoroughly disinfect the ground where the aninal lay at the time of death. The spores are very resistant both to disinfectants and the natural destructive agencies such as sunlight and drying. It is not wise to use land for grazing purposes for susceptible species upon which animals have contracted the disease. 
\$159. Preventive inoculation. Several methods of fortifying exposed animals against the infection of symptomatic anthrax virus have been proposed. In i88o, Arloing, Cornevin and Thomas demonstrated at Chaumont that animals injected with the filtrate of cultures of this virus into the jugular vein were protected against inoculation with the strong virus. It was found, however, that this method was difficult as the vein had to be exposed and the greatest care was necessary to prevent infection of extra vascular tissue in introducing and withdrawing the syringe. Later these investigators attenuated the virus by heating it to a temperature of roo to $104^{\circ} \mathrm{C}$. and injecting it into the subcutis of the slioulder. This gave a partial immunity which was reinforced after eight or ten days by a second inoculation of a virus that had been heated from 90 to $94^{\circ} \mathrm{C}$. for six hours. They injected the virus where the subcutis is quite dense, such as at the end of the tail where only local swellings would occur. This process is known as "the French method" Arloing's method or the "Lyons method."

In r888, Kitt, of the Veterinary College in Munich, after a careful investigation of the subject proposed a single injection method using a virus attenuated by heating at a temperature of from 85 to $90^{\circ} \mathrm{C}$. for six hours. A single injection of this vaccine would usually confer immunity. He further modified Arloing's method by making the injections in the shoulder region where the skin is looser and the operation easier. Later, Kitt made further important investigations concerning preventive vaccines for this disease.

In the fall of 1896 , investigations preparatory to the preparation of a black quarter vaccine were begun in the Bureau of Animal Industry at Washington by Dr. Nörgaard under the direction of Dr. D. E. Salmon. The various European methods were tried. The one finally adopted consists of a single vaccine, the Arloing principle, with Kitt's modification.

The material used for the vaccine is obtained from a fresh, blackleg tumor, by pounding the muscle tissue in a mortar with the addition of a little water and squeezing the pulp through a piece of linen cloth. The juice is spread in layers 
on plates and dried quickly at a temperature of about $35^{\circ} \mathrm{C}$. This temperature does not in the least affect the bacteria, and the dry virus obtained in this way retains a high degree of virulence for two years or longer.

When vaccine is to be prepared, the dried material is pulverized and mixed in a mortar with two parts water until it forms a semifluid homogeneous mass. This is spread in a thin layer on a suitable glass dish, and placed in an oven, the temperature of which may be regulated with exactness. The reason for mixing the dried muscle with water is to insure a quicker and more uniform attenuation. The temperature of the oven is previously brought up to $95^{\circ}$ to $99^{\circ} \mathrm{C}$., and the virus is allowed to remain in it for six hours. When removed it appears as a brownish scale, which is easily detached from the dish. This scale is pulverized and put up in packets containing so doses each. Before it is used, it is mixed with Io c.c. of water filtered and the filtrate injected in doses of one cubic centimeter.

\section{REFERENCES.}

I. Fisher. Blackleg in Kansas, and protective inoculation. Kan. State Agric. Exper. Station. Igoi.

2. LEwis Symptomatic anthrax. Bulletin No. 27. Oklahoma State Agruc. Exper. Station. 1897.

3. MAvo. Blackleg. Bulletin No.69. Kan. Stale Agric. Expt. Station. 1897, p. 108.

4. NÖRGAARD. Blackleg in the Uuited States and the distribution of vaccine by the Bureau of Animal Industry. Annual Report of the Bureau of Annual Industry, U. S. Dept. Agric. ISgS.

5. NörgAARD. Blackleg. Its nature, cause, and prevention. Ibid. Circular No. 23. U. S. Bureau of Animal Industry. 1898.

6. PETERS Blackleg. Its nature, cause, and prevention. Bulletin No. 65. Neb. State Agric. Exper. Station. I9oo.

7. SAm MoN. Black quarter. Anmual Report Bureau of Animal Industry. U.S. Dept. of Agricullure. I893-4. 
CHAPTER V.

\section{DISEASES CAUSED BY FUNGI.}

\section{ACTINOMYCOSIS.}

Synomyms. Lumpy jaw; Wooden tongue; Big head.

s6o. Characterization. Actinomycosis belongs to the class of affections known as the infections granulomata. It is a chronic disease determined by the presence of a specific cause - the ray fungus - which by irritation stimulates the formation of new growths consisting of round cells, epithelioid cells, giant cells and fibrous tissue. The neoplasms appear as tumors having either a tendency to develop into large and hard masses or to suppurate. It has been suggested that the suppuration was due to a secondary infection by pyogenic bacteria.

Cattle (genus Bos) are most often attacked. Men are susceptible but the disease is rarely found in the human subject. Horses, dogs, pigs, sheep and elephants are slightly susceptible and a few cases have been reported in each. Other species seem to be immune.

$\$$ I6r. History. The early history of this disease is quite obscure. Prior to the discovery of its specific cause it was much confused with other diseases resembling it more or less closely in certain gross appearance. It was designated by a variety of names, the more common of which are swelled head, lumpy jaw, big head, fibroma, sarcoma and osteosarcoma. It is popularly known in the United States as "lumpy jaw' aud in Europe as "wooden tongue." The popular names were probably suggested by the character of the lesions which differ to a marked degree. It was recognized as a specific disease by Rivolta in 1868 , by Perroncito in 1875 and by Bollinger in I877. The ray fungus was undoubtedly observed prior to this 
by Lebert and Robin both of whom failed to recognize it as a vegetable parasite.

The fungus was carefully described by Dr. Harz, a botanist, who gave it the name actinomyces or ray fungus. Bollinger was the first to carefully study the disease in cattle and to demonstrate the power of the ray fungus to produce disease. With this discovery of Bollinger in 1877 , actinomycosis became recognized as a definite, specific disease which could in most cases at least be differentiated from the other affections with which it had hitherto been confused. In 1845 , von Langenbeck of Kiel observed and made drawings of peculiar bodies in a case of vertebral caries in man which it is now believed were rosettes of the ray fungus. In 1878 Israel demonstrated the disease in man. Since that time it has been carefully studied and described by a number of investigators.

\$ 162. Geographical distribution. Actinomycosis is quite widely distributed throughout North and South America and Europe. It is much more prevalent in certain countries and districts than in others. The observation has been made that animals pastured upon low lands and in river valleys are more liable to contract it than those feeding upon high and dry ground. It has also been noted that cattle fed upon rough or coarse forage are more prone to the disease on account of abrasions of the buccal mucosa than those kept upon less harsh food.

It is very difficult to procure reliable statistics concerning the extent to which it occurs. The observations, which have been made at the union stock yards, Chicago, show one case of actinomycosis in from 1600 to 1700 cattle. The statistics from the abattoirs in Berlin show one case to 4250 cattle and one in 8000 pigs. These figures do not, however, indicate the extent of the disease among the farm animals as they are collected from those animals presented for slaughter only. At the clinic of the New York State Veterinary College there is presented for treatment a very few cases each year. In the Mississippi valley and in the south-west it seems to be more prevalent than it is east of the Alleghany Mountains. 
\$163. Etiology. Actinomycosis is produced by a fungus-Cladothrix actinomyces commonly known as the "ray fungus." The disease is the result of its multiplying in the tissues and not from the elaboration of a toxin. The fungus appears in minute, yellowish granules in the lesions. IIhen examined microscopically, these granules are found to be made up of rosettes varying in size from 10 to $200 \mu$ in diameter. The average size

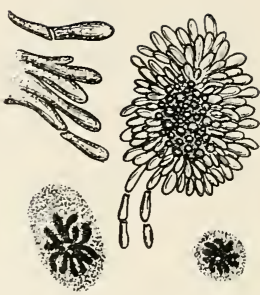

FIG. 44. The ray fungus. ranging from 30 to $40 \mu$. This fungus can be cultivated on artificial media. It stains somewhat feebly with the aniline dỳes.

The rosettes are composed of a number of club-shaped structures (rays), radiating from the central mass which is composed of the mycelial part of the fungus. The club-shaped bodies vary in size but usually they are from $\mathrm{I}$ to $10 \mu$ long. The rays are connected with the central portion by fine thread-like structures which are not readily demonstrated. In tearing or crushing the rosette, the clubs break off at or near their junction with the mycelial threads. Some investigators have mentioned a polymorphous form of actinomyces in which coccoid and rod-shaped structures are found. These are doubtless the ends of the clubs which first appear in focusing on a rosette.

The mycelial threads are wider in some portions than in others. In the narrowest places the walls seem to touch each other. Whether this irregularity is natural or the result of twisting the mycelial thread is not determined. The mycelium is much more difficult to stain than the clubs. In some cases it seems to branch. The filaments can rarely be seen in the rosettes as they occur in the suppurating lesions.

The natural habitat of this fungus is said to be on certain plants. According to Brazzola, they vegetate on the grasses chiefly on Hordeum murinum. He discovered quantities of the fungi between the vegetable fibres of barley which were imbedded in the gums of animals. Johne, Piana, Bostroem and 
others liave found it on the awns of corn which were imbedded in the tonsils of pigs and in the tongues of cattle. Mayo states, after making a careful study of this disease, that the actinomyces are probably a degenerate form of some fungus which grows naturally upon food stuffs or grain. Bostroem entertains the view that they develop exclusively on grain, particularly on the awns of barley.

\$64. Infection. While actinomycosis is an infectious disease it does not seem to be transmitted directly from one aninal to another. Numerous investigators have tried to produce the disease by inoculating cattle, calves, sheep, goats, pigs, dogs, cats, rabbits and guinea-pigs with actinomycotic lesions. The results have alnıst invariably been negative when pus was used, but the disease has developed after inoculating cattle with pieces of tissue containing the fungus in its vegetating state.

It is beliered that the infection occurs in susceptible species by the introduction of the fungus on food stuffs. The supposition is that the parasite gains entrance to the living tissues through slight wounds of the mucous nembrane of the mouth or throat and perhaps the alveoli of diseased teeth or during the shedding of the milk teeth. It multiplies and extends from the points of entrance. After the infected awns once gain lodgement, especially between the teeth, they are removed only with difficulty. The favorite points for the actinomyces to enter the tongue is on the upper surface midway between the dorsum and the tip. The lungs may be the seat of primary infection due to the inhalation of the fungus. The disease has been rarely observed primarily in the udder but frequently in the subcutaneous tissue about the head. In man the source of infection is more ohscure. Many cases have been reported where the individuals did not come in contact with diseased animals and who were not occupied in agricultural pursuits or in handling grain but who were glaziers, tailors and various shop tenders. There are a few cases reported, however, where the circumstantial evidence points to direct infection from diseased animals.

The present knowledge of this fungus indicates that it 
must attain to a certain stage or period in its development before it will live and multiply in living animal tissues. It has been observed that, as a rule, cattle become infected when they are kept upon dry food.

\$65. Symptoms. Actinomycosis is manifested by a firm swelling or tumor usually situated in the region of the head or throat. It is first recognized as a slight swelling of the affected part resembling somewhat the result of a bruise. It is stated by those who have had much experience with the disease that many cases of actinomycosis seen to be caused by blows or injuries received while struggling in stanchions. The enlargement gradually increases in size and ordinarily it is well defined from the surrounding tissues.

Upon manipulation the tumor feels hard and dense and if it is not the result of the bulging of the adjacent bone it is usually attached to it. In the region of the throat it may be fluctuating. After a variable length of time, the tumor may soften in one or more places, rupture and discharge a rather thick, yellowish and more or less sticky, purulent substance. The discharge may continue or, as often happens, the opening heals temporarily only to discharge again. The discharge often takes place into the cavity of the mouth or throat. Sometimes the neoplastic tissue increases in amount until it gradually forces its way through the opening resembling somewhat a cauliflower in appearance. The actinonycotic growth frequently increases rapidly in size after it has discharged. In later stages the teeth may become ulcerated and loose.

When the tongue is affected the animal finds it difficult to eat. The organ is swollen and in advanced cases hangs from the month with abundant salivation. When the pharynx is affected there is difficulty in swallowing and when the larynx is attacked there is difficulty in breathing. In this country the tumor is most frequently seen on the external surface of the jaw. It is stated by Salmon that it usually begins in the connective tissue beneath the skin but soon extends to the bone which it penetrates. Actinomycosis of the cervical vertebrae may cause spinal paralysis. When the lungs are attacked the animal may present the appearance of one suffering from a chronic pulmonary disease such as tuberculosis. 
Actinomycosis is not a rapidly fatal disease. Animals rarely if ever die from its immediate effects. The length of time during which they survive depends very largely upon the location of the tumor and the rapidity of its development. If the tumor is situated where it does not interfere seriously with prehension, mastication or swallowing of food or where it does not occlude or press upon the respiratory passages the animal usually survives for several years. When death occurs it is usually due to inanition, the animal being unable to take sufficient food, although the drain upon the system by the long continued discharge of pus must be severe. Mayo reports several cases where the disease was watched for five or six years and where it would probably have continued several years more had the animals not been destroyed. Most animals which become affected with actinomycosis are either destroyed, treated or slaughtered for beef in the early stages of the disease.

I66. Morbid anatomy. The actinomycotic tumors have in or near their centers rosettes of the ray fungus surrounded by cells. The newly formed tissue consists principally of epithelioid and spindle shaped, connective tissue cells, among which giant cells may appear. As these cells increase in number they press against the surrounding tissues producing the hard and dense tumors. This is especially true when they are located in the connective tissue. In certain other positions, such as the liver, the inflammatory cells are surrounded by a fibrous tissue framework which gives to the lesion a honey comb appearance. On section a disagreeable "nutty" odor is given off which Mayo considers to be quite characteristic of the disease. The outside of the tumor is usually composed of a dense layer of fibrous connective tissue. Extending from the periphery toward the center, the tissue becomes less dense and is composed largely of epithelioid cells. In the softer tissue there are often cavities of greater or less size filled with a viscid, purulent substance in which the small, yellowish granules of the ray fungus can be found. If this pus is spread in a thin layer on a smooth surface granules composed of this fungus growth can often be seen with the 
unaided eye. These pus cavities are usually connected with each other by small sinuses but sometimes they are separated by bands of fibrous tissue.

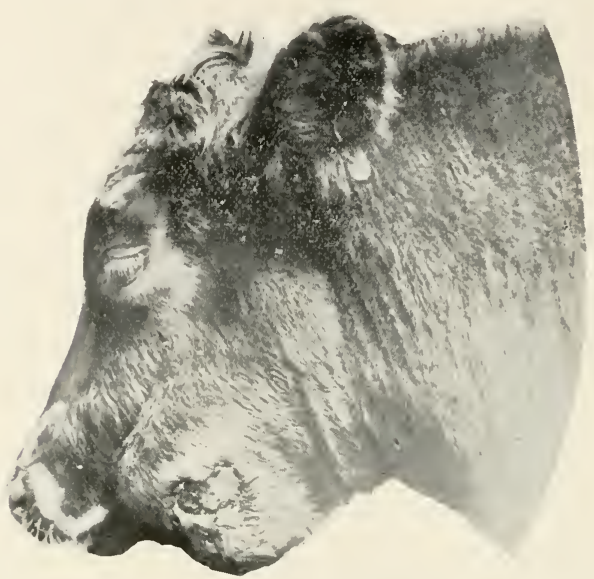

FIG. 45. Head of a steer with actinomycosis of the lower jaw. (Photographed by Hopkins.)

If the disease is in the bone, usually in the head, as it is where the specific organism gains entrance and begins to grow in the interior of the bone, the bone tissue about the organism becomes in places disintegrated and absorbed and pockets are formed containing the fungus. While the interior of the bone is being broken down and absorbed by the action of the actinomycotic growth within, its diameter is being increased by the deposition of new tissue until it may becone several times its normal size.

The disease spreads within the body in most cases by gradual invasion of the tissues surrounding the infected point. At the seat of infection, minute, inflammatory points appear which extend at their periphery and unite to form larger areas of diseased tissue. These masses tend to extend in one direction and to heal in another leaving behind bands of cicatricial 
tissue. The process usually differs widely from that of a simple inflammation. In its progress the disease shows no preference for structures but invades one tissue after another so that all may be involved alike.

The lymplatics show no constant tendency to become involved. Metastasis occurs in a very small proportion of the cases. When it does, as reported by Ponfick, large areas may be simultaneously affected. He reports a case in the human subject in which the left jugular vein was perforated by a mass of the fungi resulting in the formation of actinomycotic infarcts in the lungs, spleen, brain, and heart.

In cattle, actinomycosis usually appears in one or more of the following locations, viz.: (I) in the maxillary bones it generally results in large tumors. Actinomycosis of the jaw usually commences with flat granulations of the gums and mucous membranes in the neighborhood of the

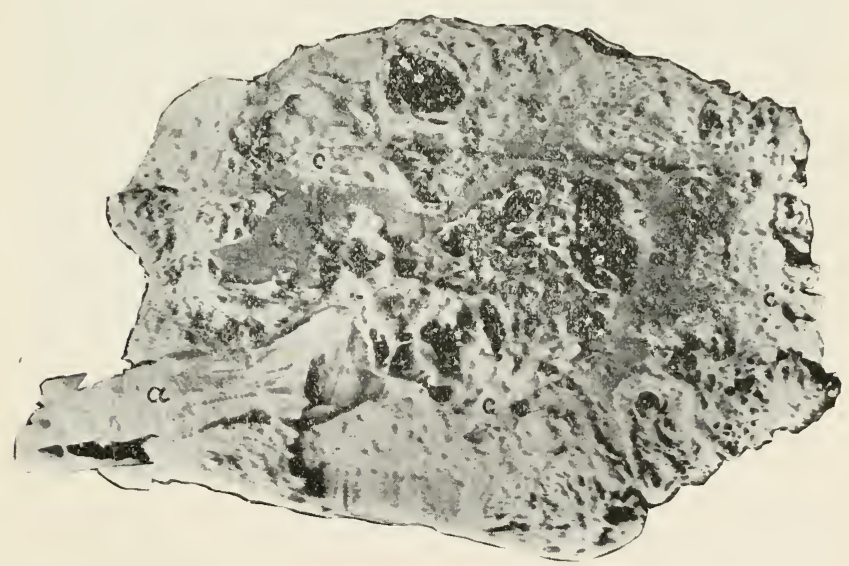

FIG. 46. Photograph of a section through an actinomycotic jaze. (a) tooth, (b) bone, (c) actinomycotic tissue.

teeth and spreads finally to the medullary tissues of the bone and to the periostem soon giving rise to the osseous tumor. From the maxillary bone the disease may advance either to 
the subcutaneous connective tissue and the skin or to the oral cavity in the direction of the molar teeth which become displaced. (2) In the tongue. When the disease appears in this organ it takes the form of an indurating glossitis. The tongue becomes thickly sprinkled with round or oval, lard, fibrous nodules which finally become purulent or chalky at the center. Around these there is a considerable increase of connective tissue which leads to the atrophy of the muscle fibers. Upon section the tongue is found to be hard and often gritty. The indurated tongue is often eroded from friction and various

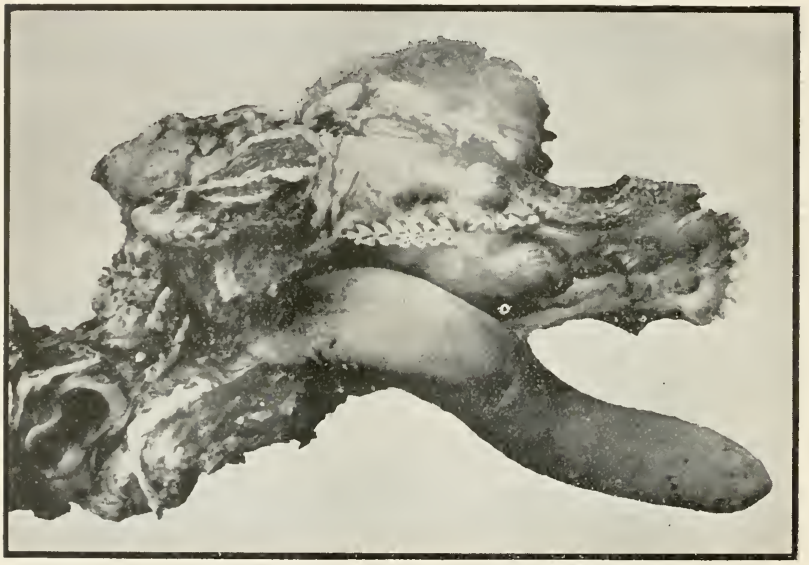

FIG. 47. Actinomycosis of the jaw. Photographed by Hopkins. deformities of this organ are reported. (3) In the pharynx the disease usually takes the form of soft polypoid or fungoid nodules or lumps with a smooth surface and short peduncle. These nodules vary in size often reaching that of a goose's egg. These polypoid growths may cause great difficulty in swallowing and likewise interfere with respiration. Tumors of this kind may form in the oesophagus or trachea. Rarely actinomycotic growths occur at other places in the alimentary tract. There are some cases in which the lesions are 
not restricted to the digestive tract. (4) In the skin and subcutaneous tissue the lesions are found chiefly on the head and neck. They usually consist of firm nodules from the size of a hazel nut to that of a man's fist or even larger. Sometimes these nodules are pedunculated and at others they are attached to the skin by a broad base. Instead of the hard tumor there may occur soft granular fungoid proliferations covered with a brown crust or with a purulent secretion. At other times mininte nodules appear in these proliferations and the skin becomes thickened and indurated. However, the skin lesions

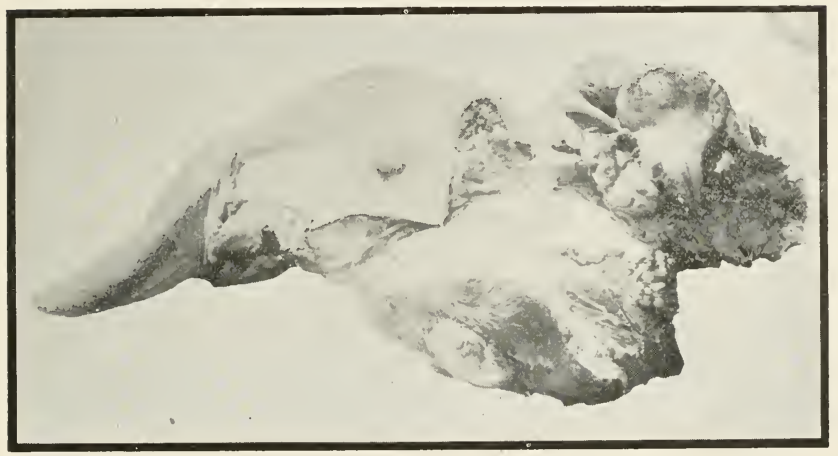

Fig 4S. Actinomycosis of the tongue, "wooden tongue." |Photographed by Hopkins.)

may become very large. In this organ, the disease may be either primary or secondary. (5) In the lymph glands actinomycosis often appears in the lymph glands of the head, larynx and pharynx. The parotid and submaxillary glands are sometimes involved as secondary infections. It is reported that the sub-parotid glands are most frequently affected. (6) In the lungs the lesions vary. They may consist of firm, somewhat yellowish nodules which eventually become calcareous in their center and vary in size from mere specks to that of a pea. This form is spoken of as miliary actinomycosis. In the second form the actinomycotic foci soften and 
become filled with a gray muco-purulent fluid. The lesion may spread to the pleurae and even reach to the surface of the body by penetrating through the thoracic wall. The bronchial glands and the mucosa of the air passages may also become affected. (7) Actinomycosis has been reported rarely as attacking the udder, spermatic cord, brain, spleen, liver, muscle, diaphragm, peritoneum, inguinal glands, vagina, uterus and cervical vertebrae.

It is stated by Salmon that in England the disease appears most often in the tongue, in Denmark the soft parts of the head are affected most frequently while in some parts of Germany it is most frequently found in the plarynx. In the United States it nswally appears in the lower jaw. In man, as already stated, actinomycosis rarely occurs. Here, as in cattle, the appearance of the lesions vary according to the nature of the part affected. In some cases the lesions closely resemble those of chronic inflammation but in others such as the liver or skin they are often characteristic. In the lungs the lesions have frequently been mistaken for tubercle. Usually the disease affects the head and if the maxillary bones are attacked the teeth are usually lost.

Actinomycosis in saine. Actinomycosis appears in this species in the lower jaw, larynx, lungs, wounds caused by castration, in the mamnary gland, muscle and bones. The character of the lesions does not differ to any marked extent in swine from those in cattle or man. In case of bone infection purulent cavities and sinuses are formed in which the yellow granules of the fungus occur. It is reported that occasionally the pig suffers from generalized actinonycosis. Duncker has found in the muscles of the pig a variety of the ray fungus which has been called Actinomyces muscolonum suis, to distingtiish it if possible from the bovine species. Its relation to the actinomyces boris has not been clearly deternined. It is reported to have been found frequently.

Actinomycosis in horses and sheep. In the horse, actinomycosis of the bones, tongue, trachea, spermatic cord and submaxillary glands has been observed. The disease is reported to have been mistaken for glanders. The affection 
known as scirrhous cord seems to be due in rare cases to an infection by the ray fungus. One such case has occurred in the clinic of this institution. A very few cases of this disease have been reported in sheep the lesions being restricted to the lungs or muscles.

$\$$ i67. Differential diagnosis. In cattle actinomycosis is to be differentiated ( $\mathrm{I}$ ) from tuberculosis, especially of the lungs, glands about the throat, head and the udder, (2) various forms of glossitis, polypoid growths in the pharynx, fibroma, sarcoma and osteosarcoma of the jaw, parotitis and cellulitis. The writer has seen a few cases of bacterial infection of the maxillary glands giving rise to the formation of large quantities of caseous matter which caused swelling and firmmess suggestive of actinomycotic tumors. In one instance a specimen reported to be actinomycosis was examined and found to contain a piece of bone about three inches long which had become wedged between the teeth and cheek and surrounded by inflammatory tissues. Fröhner calls attention to contagious plenro-pneumonia and foot and month disease as possibly being mistaken for this disease.

In making a positive diagnosis of actinomycosis it is necessary to make a microscopic examination of some of the diseased tissue or of the discharged pus in which the ray fungus may be found if the disease is actinomycosis. It is impossible to obtain this positive proof from the living animal when the disease is situated in the internal organs. With these it is necessary to depend largely upon the history and general condition of the animal. In preparing the discharged pus for a microscopic examination it is usually sufficient to crush one or more of the yellowish granules between a slide and cover-glass. It is of advantage to wash it with a dilute solution of caustic soda to clear away the pus cells. The rosetts are easily recognized with a low magnification.

In man, actinomycosis is to be differentiated from certain forms of tuberculosis and the Madura foot disease which was described by Carter, in 1860 , as a "fungous disease." 'This is a chronic, locally spreading inflammation of the foot, rarely of the hand, causing the destruction of the part involved and 
giving rise to a great overgrowth of connective tissue. Mycetoma almost invariably attacks the hand or foot and according to Carter there are no secondary deposits in the viscera. In actinomycosis the extremities are rarely attacked and the viscera are often the seat of the disease ; further the mycetoma is a disease of hot climates while actinomycosis is a disease of the temperate latitudes. The fungus of the two affections seem to be closely related but as yet their identity has not been established.

$\$$ I68. Specific treatment. The investigations of Thomassen, Nocard, and Nörgaard and the trials of a large number of veterinarians have proved very conclusively the specific, curative effect of iodide of potassium. According to Salmon the iodide of potassium is given in doses of from $\mathrm{I} / 2$ to $2 \mathrm{I} / 2$ drams dissolved in water and administered in a drench, once a day. The dose should vary somewhat with the size of the animal and with the effects that are produced. If the dose is sufficiently large there appear signs of iodism in the course of a week or ten days. The skin becomes scurfy, the eyes moistened, nasal catarrh and loss of appetite. When these symptoms appear the medicine may be suspended for a few days and afterwards resumed in the same dose. The cure requires. from three to six weeks treatment. Some animals do not improve with the administration of iodide of potassium and these are generally the ones which show no signs of iodism.

If there is no sign of improvement after the animals have been treated four or five weeks and the medicine has been given in as large doses as appear desirable, it is an indication that the particular animal is not susceptible to the curative effects of the drug and the treatment should be abandoned.

It is not, however, advisable to administer iodide of potassium to milch cows, as it will considerably reduce the milk secretion or stop it altogether. Furthermore, a great part of the drug is excreted through the milk making it unfit for use. It should not be given to animals in advanced pregnancy, as there is danger of producing abortion.

$\$$ r6g. Sanitary considerations. The literature upon this subject is largely to the effect that actinomycosis is rarely 
if ever either contagious or infectious in the sense that it can be transmitted from one animal to another or from one of the lower animals to man. There seems to be no indisputable case on record of such a transmission although a few cases are very suggestive. It is the opinion of most pathologists that when the disease is restricted to small tumors and these are localized, that the affected parts should be destroyed but the remainder of the carcass may be used for human consumption.

In Bulletin No. 2, of the Board of Live Stock Commissioners of Illinois, published in $\mathrm{I} 89 \mathrm{I}$ is the report of the somewhat famous trial in the Peoria county circuit court of the case of J. B. Greenhut et al. vs. John M. Pearson et al. to recover damages for the rejection and destruction of certain actinomycotic cattle, in which is given the testimony of a large number of distinguished reterinarians and sanitarians concerning the wholesomeness of the meat of cattle affected with this disease. Althougl at that time there was a strong popular sentiment against the use of such animals, the jury after a forty hours' consideration reported their inability to agree and were discharged by the court. The most conspicuous feature of this eridence was the inability of the witnesses to produce satisfactory evidence of the communicability of the disease from animal to man. This evidence did much to show that the danger from this disease in eating neat of affected animals was after all a matter of opinion, fear or sentiment rather than a demonstrated fact. Mayo, in his bulletin upon the subject, states that there is no danger of persons contracting this disease from eating the flesh of affected animals provided the risibly diseased portions are removed.

\section{REFERENCES.}

I. Bostrokn. Untersuchungen über des Aktinomykose des Menschen. Beitrag. zur path. Anat. u. zur allgr. Pathologie. Bd. IX. (I89I) S. I.

2. ISRAE: Neue Beobachtungen auf dun Gebeite des Mykosen des Menschen. Virchow's Archives. Bd. LXXVI. (1878) S. 11.

3. Mayo. Actinomy cosis bovis or "lumpy-jaw." Bulletin No. 38 , Kansas State Agric. Exp. Station. 1892.

4. Nocard. Notes sur l'actinomycose des animaux. Recueil de lled. Veter. Vol. LXIX. (IS92) p. 167. 
5. Salmon. Treatment of lumpy-jaw or actinomycosis in cattle. Bulletin No. 2, C. S. Bureau of Animal Industry. 1893.

6. Saluon. Actinomycosis or lumpy-jaw. Annual Report, Bureau of Animal Industry. I $893-4$, p. 85.

7. WOLFF AND ISRAEI, Ueber Reincultur des Actinomyces und seine Uebertragbarkeit anf Thiere. Virch. Arch. Bd. XXVI. (IS9I) S. II.

\section{LEECHES.}

S I70. Characterization. "Leeches" or "Leeching" is an infectious disease quite prevalent anong the horse kind in Florida with lesions localized on the skin or the mucosa of the head. It is thought by many that this affection is identical with the disease known as bursattee* in India.

This disease is known by a variety of names such as: barsati, barsáti, barsattee, barsatti, bausette, bursati, bursatie, bursatti, bursautee, bursautie, bursauttie, bursottee, burusatee and burusanttee. These names have been derived from the Indian word burus or bursat, meaning rain or rain sore, it having been supposed that the malady was associated with the rainy season.

Dr. L. C. Neal, of Florida, described it as affecting horses and cattle. He believes it to be peculiar to that section, where he states, it is common and very fatal to horses and mules. There are hundreds of ponds in the central portion of the state around the margins of which there is usually a belt of grassy prairie, water grass and water lilies. Into these grassy places the horses, mules and cows often go during the summer and feed all day in the water. He adds, "after a varying exposure to the influence, or whatever it may be called, of the 'pond,' a slight lump or elevation of the skin may be found on some part of the body that has been submerged. To the touch it will feel as if a grain of shot were lodged beneath the skin. In eight or ten days the skin sloughs off centrally over this hard spot, leaving a bloody, bruised-like surface. This rapidly

*It seems to be true that an entirely different affection is known by the same name in the northern portion of the United States. The term "Leeches" is also applied to the condition following the invasion of the liver fluke (Fasciola hepatica). 
grows in size till in a few weeks there is a raw surface from 4 inches to a foot square. This oozes blood and serum, but 110 pus. An examination will show usually a mass of yellow, gritty growth, coral like in shape, embedded in a mass of bruised, bloody tissue, dark in color and the edges roughened, elevated above the skin, and the skin decaying at the outside of the ulcer. The leech invades most any tissue, but seems most common on the legs, abdomen, and sides. Occasionally it is found in the head. The invaded tissues decay slowly and apparently without pain. I have seen hoofs cut off, the abdomen opened, the eves eaten out and the teeth destroyed."

In this country the disease has not attracted very nuch attention, nor has it been considered of much economic importance. An explanation for this may be offered on account of its seemingly noncontagious character and that it has been thought to be confined to comparatively limited areas, and that the animals, although infected, may yet be utilized for some purposes. On account of the chronic course of the disease the affected animals from a sentiment of mercy are often killed before the disease can terminate fatally.

Although this affection presents many points of similarity to the one found in India, the question of their complete identity ought to be held in abeyance until a more thorough investigation can be made.

In a recent publication, Dawson, of the Florida Experiment Station, states 'that 'leeches' or bursattee is a common disease in Florida, which manifests itself in the formation of tumor-growths which have some of the characters of actinomycotic tumors. Its structure is fibrous, and contains many sinuses, which discharge a bloody, honey-like fluid. It is a fatal, infectious disease, which has its origin in the skin and finally penetrates all the tissues. Here and there in the tumor tissue yellow bodies with root like projections may be found. These bodies are called 'leeches' by the natives. They consist of the mycelia of the fungus which causes the disease. The only remedy is the complete removal of the tumor and adjacent tissue at once. The application of caustics and disinfectants make matters infinitely worse, as they 
stimulate the tissues to renewed growth-activity. In Florida the disease affects only the genus equus."

S I7I. History. In 1896 , some of the diseased tissue from cases of this affection, in Florida, were sent to the Bureau of Animal Industry for investigation. They were studied by Dr. P. A. Fish who made an extended report on the results of his findings. He also gave a very complete review of the literature.

A summary of the literature shows that among the old theories 'Leeches' 'was believed to be a blood disease in many ways not unlike syphilis, scrofula and farcy. The "fly theory" of the causation and dissemination of bursattee was entertained by the natives of India as early as I820. Jackson, in 1842 , seems to have been the first to believe that there was any connection between the disease and a fungus.

Hodgson, in I 853, referred to the sores as cancerous ulcers and Hart in 1872, was strongly inclined to pronounce it cancer, although he could not confirm this view structurally by microscopic examination of the tissue. It seems to be generally accepted that the disease is peculiar to the Tropics, but cases liave been reported in Kansas and Minnesota in the United States, not only during the summer months, but when the thermometer registered below zero. We might also expect that the disease would exist in Mexico and Central and South American countries, where the conditions of temperature and moisture are favorable.

Reports show that a high temperature is essential for the development of the disease, although exceptional cases are noted as occurring during the cold season. Moisture does not seen to be necessary, since many cases develop when the season is dry. It is, however, an important factor. Statistics show that cases are more numerous and that the disease assumes a more aggravated form during the wet season.

In India, native as well as foreign bred horses are susceptible, but according to some writers, none of the other equine species are affected.

In the United States mules and cattle are said to develop it, but not as readily as the horse. Outbreaks among cattle are 
comparatively rare. Thin-skinned animals are more susceptible than thick-skinned ones. Some discrepancy of opinion exists as to the kind of horses most likely to take the disease (assuming that bursattee and leeches are similar). Neal states that only horses of good blood leech, and the Cuban and Texan ponies are as a rule exempt. Anderson states that it is the coarsely bred and hard-rorked horses that are the most susceptible. The well-bred ones, having the advantage of good hygienic surroundings, rarely contract it.

$\$ 172$. Geographical distribution. Bursattee has been reported from Burmah.and Hindoostan. It is thought that the prevalence of the disease is associated with the principal river systems of India. In the hilly, rocky and consequently drier districts there is a very noticeable diminution or absence of it.

Outside of India there seems to have been no cases of this malady reported except in the United States, unless upon further investigation certain mycotic diseases, which have been described in Europe, should prove to be identical with it.

Lyford (I866) reported it in Minnesota, Anderson (1889) in Kansas and Alabama, and Neal (I857) and Bitting (I894) in Florida. The latter writer states, that it is "now known all over the United States except in that region lying east of Alleghany mountains and north of the Potomac river." A few cases have been presented for treatment in the clinic of the New York State Veterinary College.

$\$$ I73. Etiology. Jackson suggested, in I $\delta_{42}$, that the disease might be related to a fungus or to a regetable parasite. Collins, in 1874 , expressed a similar belief. F. Smith, in 1879 and $188_{4}$, seems to have been the first to have worked along this line. He was able to find fungi in every fresh specimen of the sores that he examined. Steel, in r88r, also found fungal elements in these sores. T. Smith, in I893, examined some alcoholic material and gave expression to the belief that the disease was caused by a fungus. Fish, in I896, found a fungus embedded in the diseased tissue. He did not name it neither did he obtain it in pure culture, but his illustrations are very clear in showing the existence of a fungus. Fish gives in detail the methods he employed. It is to his work 
that we are indebted for the more careful description of the morbid changes.

\section{S I74. Morbid anatomy.} As a rule the lesions are near the surface. Where the diseased portion has become well developed there is usually a more or less complete detachment of the central inflammatory growth from the surrounding tissue. This nodular or ' kunkur', growth may vary in its density according to the stage of its development.

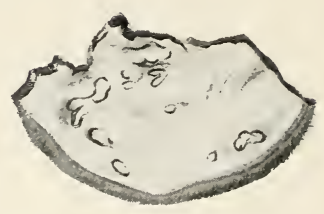

Fig. 49. A piece cut from the lip of an affected horse, showing sezieral diseased foci. (Somewhat reduced.) (Fish.)

During the earlier stages it is soft and easily cut; later it becomes firmer and ultimately assumes a hard or "gritty" character.

In cutting sections it is generally the exception to cut through the nodule or kunkur evenly and to have it retain its proper relation with the other parts. Even if successful in cutting, the nodule drops out during some of the later processes. In the specimens examined the lesions were confined entirely to the skin and subcutaneous tissue; no traces of muscular or glandular structure were observed. Around the central portion of the inflammatory growth there is a zone of lencocytes of the mononuclear and polynuclear varieties, the latter predominating. They are embedded in an abundant stroma of connective tissue which is in a greater or less stage of degeneration. The central portion of the zone is in some cases very closely packed with the leucocytes, while toward the periphery they are more loosely arranged and cause a marked irregularity of the margin from their uneven drifting into the tissue beyond. There is generally one and perhaps more points where this infiltration occurs quite extensiveiy. In some of the preparations the wandering cells have been traced as far as the surface of the epidermis.

Occasionally there may be found a narrow area at the periphery of the nodule, as seen in cross section, which is lighter in 
color and less dense in texture than the central mass, evidently

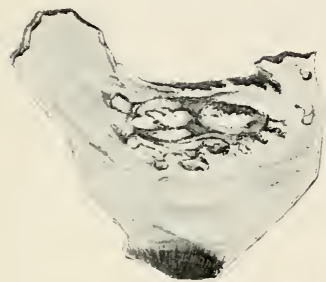

Fig. 50. From the same lip, stead of presenting the usual but shows a larger infected area. flattened appearance, was irreg(Fish.) ularly cylindrical. Although the condition was not observed, it is not impossible that the hyphae of the fungus may develop to such an extent as to compress or actually penetrate the walls of the ressels, causing inflammatory changes sufficient to permit, in the course of time, a disorganization or absorption of a portion of the ressel itself, and that ultimately it may become incorporated in the nodule.

The nodules are generally irregularly cone shaped and are of variable size. In section they reveal a very dense structure, the framework of which forms a close reticulum.

Within the meshes are what appear to be leucocytes in various stages of disintegration, and free nuclei. Among these, at places, there can be seen small bodies of nearly the same size as the nuclei and taking the stains in the same way, but differing in form. At one portion of its circumference the substance of the body is seen to draw itself toward a point and in favorable preparations that point

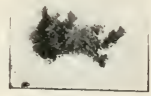

FIG. 51. $A n$ isolated nodule showing the characteristic roughened, coral-like appearance of the has been followed some little distance as a mass. (Fish.) delicate filament. In most cases the filament remains unstained, or, as observed in a Gram-eosin preparation, the club end may stain blue and the filament red. Exceptionally one may find a clear area or vacuole in one of the clubs. From the fact that the filament is not usually traceable to its central 
connection a more or less flagellate appearance is given to the fungus, which represents a condition not believed to exist.

Not infrequently small spherical bodies are found not far from the clubs which take the stain readily and whose size is sufficiently small to admit of the possibility of their being spores. The free ends of many of the clubs point toward the periplery of the nodule, but this is not a constant feature.

The framework of the nodule stains very slightly or not at all and shows among the enmeshed corpuscles as a very irregular, distorted and somewhat glistening network. It is this portion of the nodule that gives the hard, gritty feeling, and is probably due to a greater or less deposition of lime salts along the reticulum. It appears that the framework of the nodule is composed of a mycelial net, which in the course of development has become more or less calcified.

As a result of the treat-

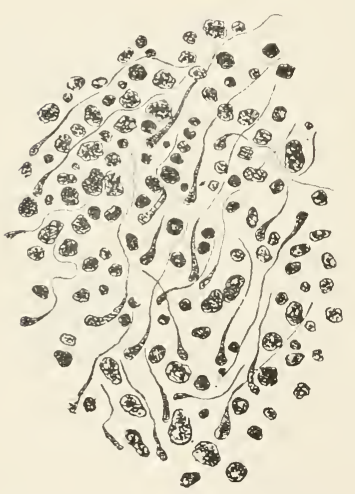

FIG. 52. The fungus. Toluidin blue preparation. (Fish.) ment of the nodules with the 10 per cent cold solution of caustic potash, a very profuse and intricately branched fungus became apparent. The branching is of an irregular order. In places there is seen in the filament a central axis, which takes the stain, and around this appears a transparent or hyaline sheath of varying size.

In certain of the teased preparations (Biondi-Ehrlich stain) the wall of the filament. instead of being smooth and homogeneous, appears roughened, as if covered with very minute but nun1erous spinous processes.

In the sections of the tissue in which the fungus appeared the substance of the filament was not uniform. In places it was drawn together in an irregular manner, with intervening clear spaces of greater or less area. 


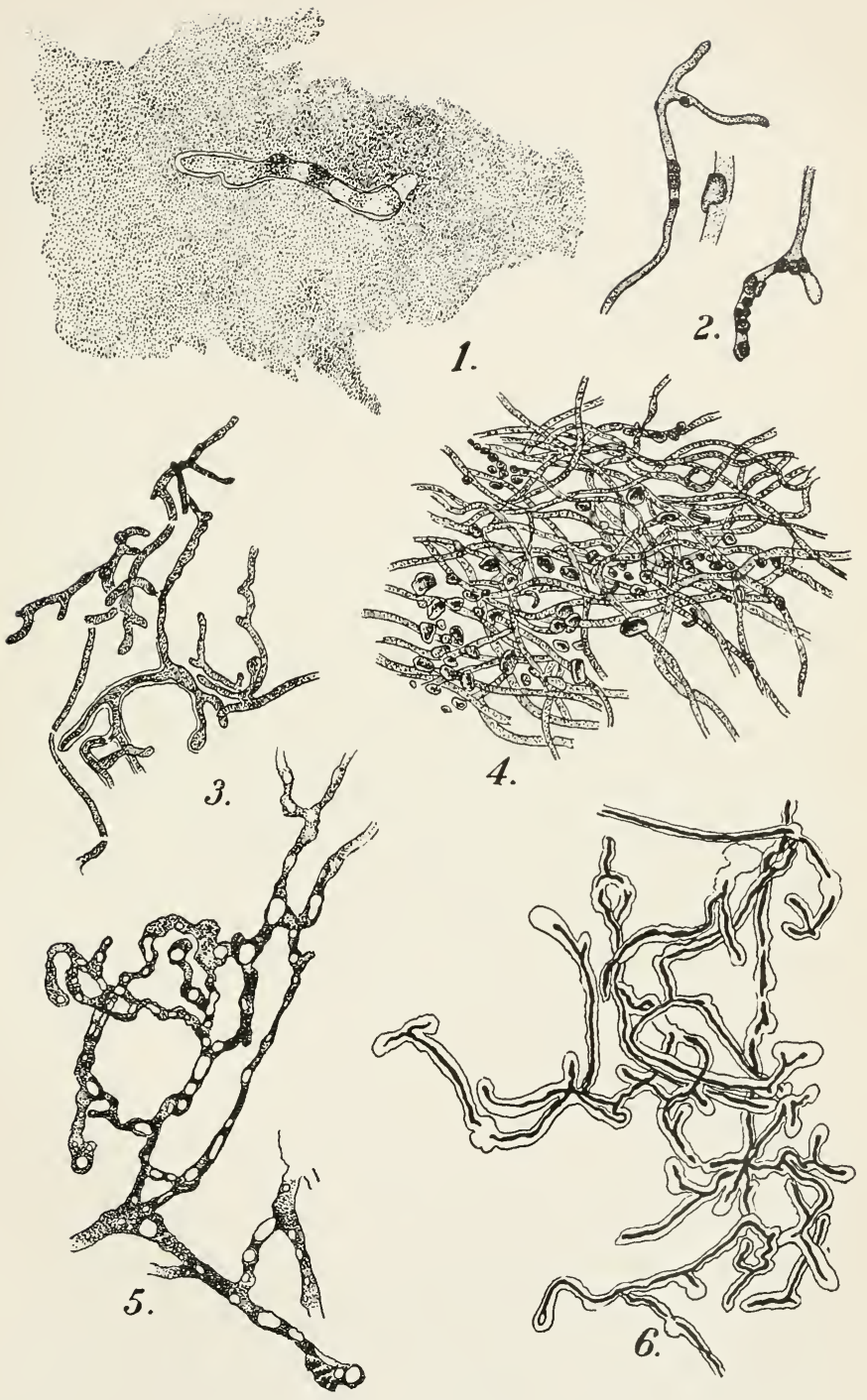

Fungus. 
8 
In some places the filaments show distinct septa, but the latter are not common. Some of the club-like endings, especially those that are elongated, show a septum at the union with the filament proper. Scattered among and coiled around the ordinary filaments there have been observed much more slender ones apparently deroid of any external sheath.

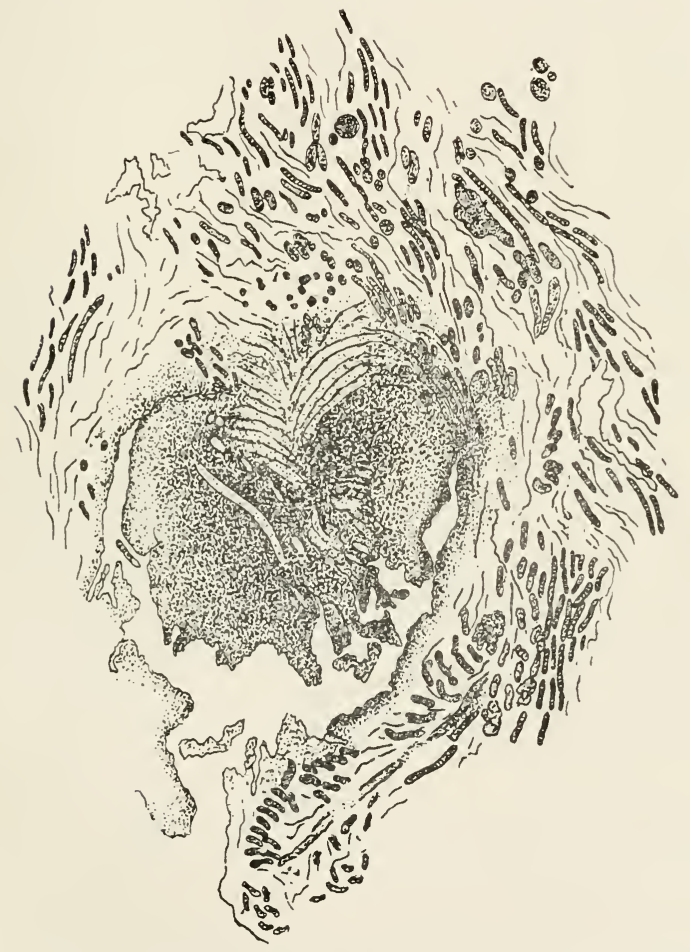

FIG. 53. A section through a nodule showing its dense texture, with a portion of its peripheral zone made up of hypha with leucocytes interspersed. Methylene blue and eosin stain. No. + ocular, smm. objective. Camera lucida. (Fish.) 
There have also been observed numerous small circular bodies of inconstant size. They have been seen lying freely in the meshes of the mycelium and also closely applied to the filaments. These bodies are not spherical, but thin and flattened, and some of them present a curved appearance, convex on the outer side and concave on the inner side. They suggest the possibility of having been closely applied to the filaments and have something of a scale-like arrangement. With possibly one exception, no trace of blood vessels has been found in the nodules.

In the circumnodular tissue pathologic conditions exist consisting of certain areas of tissue necrosis.

There is an infiltration of the connective tissue with a great number of wandering cells. In some places there are well-defined nests in the stroma of the connective tissue, simulating, perhaps, a cancerous appearance. The character of the cells, which present a curiously racuolated condition, would, however, tend to eliminate this view. The vacuoles vary in number and size, the average number being Io to i 2 in a cell.

In some preparations numerous lencocytes, of the mononuclear and polynuclear variety, had drifted away from the nodule. They were for the most part elongated, and in all the nucleus or nuclei appeared to be in a healthy condition. The cells contained numerous small bodies, which took a deep orange color with the Biondi-Ehrlich stain. In places adjacent to these leucocytes there were frequently noticed a number of these small bodies apparently lying free in the tissue. No definite cell wall was distinguishable in the leucocytes.

The vacuolated cells are present in greater numbers than the heavily laden leucocytes. In the former nuclei were present and presented various phases of change. In some there is a single nucleus, which may be circular, crescentic, or in the form of a dumb-bell; in others there may be two or more nuclei which in advanced cases appear only as remnants. In extreme cases no nuclei at all are visible. The wall of the wandering cell differs from that of the leucocytes proper in possessing an appreciable thickness. This thickened boundary 
apparantly gives considerable rigidity to the cells, as nearly all of them are approximately circular in form. Their arerage diameter is about 8 microns. In one specimen there appeared to be large giant cells, measuring from 12 to 18 microns and apparently possessing quite a distinct cell wall. Within each giant cell there is some appearance of vacuolated cells, each with a single nucleus and fairly well-defined cell boundary. As many as eight or ten of these nuclei have been counted in a single giant cell. There is the possibility that these apparent giant cells are simply some of the vacuolated cells fused together, but the nuclei are well defined and take the stain very intensely, which is not commonly the case in the ordinary vacuolated cells.

The connective-tissue cells surrounding the nodule show marked signs of degeneration, their cytoplasm in most cases being extremely vacuolated. Among these connective-tissue cells, which for the most part are quite branching and elongated, is another class of cells which are in general of an oval or elliptical form. The noteworthy appearance of these cells is the presence of numerous dots in the cytoplasm which take the methylene blue and toluidin blue stains very deeply. The appearance is, indeed, very much as if the cells were filled with micrococci. These are the granule cells of Waldeyer, or still further differentiated as the plasma cells, in contradistinction to the "mastzellen" or "food" cells," which indicate an exalted degree of nutrition. The nucleus of the plasma cell takes the stain very slightly, or not at all, and is almost entirely obscured by the numerous "granules" in the cytoplasm. These cells are well differentiated by the toluidin blue stain, as they take a deep purple color, while the surrounding cells are blue.

Bitting has figured the jaw bone of a horse quite extensively affected with this disease. He believes that the lesions about the mouth result from the animal biting the affected areas on the body.

Neyrick reports finding the inflammatory growths in the lungs of an affected subject and Burke has reported them in 
the liver. There are no other lesions described in the internal organs although Neal writes that any tissue may be invaded.

5 i75. Treatment. On the ground that the fungus supposed to be the cause of this disease may be closely related to the ray fungus of actinomycosis, the use of iodide of potassium has been recommended. It is reported to be fairly successful. The efficiency of this drug as a specific needs further confirmation.

\section{DESCRIPTION OF PLATE V.}

FIG. I. Showing a young fungus embedded in the tissue (lip.)

FIG. 2. Showing scale-like bodies embracing the filaments.

FIG. 3. Showing young branches of the jungus, and in one place the transparent sheath. Hematoxylin preparation.

FIG. 4. Mycelium with scale-like bodies lying among the filaments. Hematoxylin.

Fig. 5. Vacuolated filaments. Hematoxylin.

FIG. 6. Branches showing a zvell-developed, transparent sheath. In places the axis of the branch is disconnected and occasionally there is a faint sign of a septum. Hematoxylin.

No. 4 ocular, $2 \mathrm{~mm}$. objective. Camera lucida. (After Fish)

REFEREINCES.

I. Bitring. Leeches or leeching. Bulletin No. 25, Florida Agricultural Experiment Station. I894.

2. Fish. A histological investigation of two cases of an equine mycosis, with a historicai account of a supposed similar disease called bursattee occurring in India. Annual Report, Bureau of Animal Industry, U.S. Dept. of Agriculture. I895-6. p 229. (This report contains full bibliography to literature on Bursattee. )

3. NEAL. "Leeching of horses and cattle. Annual Report, Bureau of Animal Industry, U.S. Dept of Agriculture. ${ }_{1887}-8$. p. 489 .

\section{PNEUMOMYCOSIS.}

$\$ 176$. Species of fungi. The literature contains a number of reports of cases of mycosis in the lower animals as well as in man due to infection with different moulds. The genus Aspergillus seems to infect and to produce lesions in animals more frequently than the members of other genera. In fact, Aspergillus fumigatus seems to be the only important pathogenic species. The lesions encountered as a result of its invasion are 
largely restricted to the respiratory tract. Pneumomycosis has been reported in a number of cases. Cadéac, Schneidemïhl, Friedberger and Fröhner, Ostertag and Kitt have all called attention to mycotic pneumonia. Renon considers the lesions resulting from aspergillus infection as a pseudo-tuberculosis which he would designate as "Aspergillar tuberculosis." Aspergillar pneumonia is, however, quite rare.

$\$$ 177. Description and method of cultivation of the mould. The aspergillus fungus is readily cultivated artificially. It grows on most of the ordinary culture media used in bacteriology if the reaction is acid ; it develops poorly in alkiline media. The well-known Raulin's fluid is reported to be the best medium for itscultivation, especially where the aspergillus must be isolated from mixed growths, as in the examination of sputum. Sabouraud's* solution of maltose also gives good results.

For ordinary use potato, with or without glycerin, gives excellent results. A paste made by rubbing up crumbs of stale bread in water is also a good medium. Growtl is said to be more rapid, however, in Ranlin's fluid than in any other madium, the inycelium appearing in from five to twelve hours and spores forming in from twelve to fifteen hours. The growth is first a velvety white, soon becoming a delicate bluish green, which grows darker. On Raulin's fluid it changes after some days to a dark brown. Cultures on potato retain the green color for a long time, while those on bread paste become brown.

The fungus retains its vitality in cultures for many months unimpared. Its development has been reported when inoculated from cultures three or four years old. Spores do not form in a temperature below $20^{\circ} \mathrm{C}$. and like the mycelium they require free access to oxygen for their best

*The formula recommended by Ravenel is as follows, viz.:

$$
\begin{array}{lr}
\text { Maltose, } & 3.70 \text { grams. } \\
\text { Peptone, } & 0.75 \text { grams. } \\
\text { Distilled water, } & 1 \mathrm{co} .00 \mathrm{c} \text { c. }
\end{array}
$$

To this unay be added gelatin or agar to solidify it, the latter being preferable, as the aspergillus grows best, and forms fruit best at $37^{\circ}$ to $39^{\circ} \mathrm{C}$. 
development. They measure 2.5 to $3 \mu$ in diameter. In nature the spores are widely distributed but seem to be especially abundant in grain and regetable matter. They have considerable power of resistance to heat and to chemical agents. They are killed by a temperature of $60^{\circ} \mathrm{C}$. in five and one-half hours. In moist heat and in a solution of bichloride of mercury $\mathrm{I}$ to 1,000 they are destroyed in fifteen minutes.

Aspergillus fumigatus is differentiated from other species by its color in cultures, the high temperature at which it grows, the size of its spores and by its pathogenesis. Aspergillus glaucus is the one most likely to be confounded with it. It may be differentiated from $A$. fumigatus by its ability to grow at low temperatures, its delicate green color, the large diameter of its spores-9 to i $5 \mu$-and its lack of pathogenic power.

The mode of infection is through the respiratory tract. Only a small number of the spores inspired are able to reach the alveoli, the greater number of them being arrested in the tracheal and bronchial secretions. Hildebrant has shown that, having reached the alveoli, they penetrate the epithelial lining without difficulty. Both animals and man seem to possess immunity to intestinal infection. Renon has produced it only a few times experimentally.

The aspergillus does not form a toxin. Its pathogenic power is due entirely to lesions produced by the masses of mycelium which cause a necrosis of the cells and a leucocytic reaction which diminishes the functions of the organs, the final result being an enfeebled condition of the animal and a lessened resistance to hurtful influences. When fruit hyphae can form, the myriads of spores given off by them may be carried to other parts of the organ. In this way the foci rapidly multiply and practically the entire organ becomes invaded. The opinion held by some authors that in the mould mycoses there is "no fructification or actual multiplication" of the infecting agent and that the "number of the diseased foci corresponds exactly with the number of spores introduced" is erroneous both for the disease naturally contracted as well as 
for the experimental form. In the produced lesions, fruit formation is exceptional ; but it has been observed by Renon to take place only where there is full communication with the air. It lias been observed only in the lung. It is extremely rare for aspergillosis to pass from one animal to another. The infection can only take place with the spores.

Recently Pearson and Ravenel have described a very interesting case of pneumomycosis in a cow due to A. fumigatus. As this seems to be the only carefully described case in this species of animals listed in this country their description of the symptoms and lesions is very largely incorporated here. The case was in a Jersey cow six years old. She had been in bad condition for six months prior to bringing her to the Veterinary Hospital where she was tested with tuberculin with no reaction. At this time she did not eat, was weak and depressed, respiration labored and from 40 to 60 per minute. Pulse rapid. Percussion of the chest walls gave a sound that if anything was clearer and louder than the normal percussion sound. Upon auscultation it was found that the vesicular and bronchial murmurs were considerably increased in intensity and accompanied here and there by sibilant râles. She coughed violently at times. Six days after she came to the hospital the breathing became more rapid and difficult and the pulse rery much accelerated. The animal did not eat, grew weak rapidly and died four days later, or ten days after admission to the hospital.

$\leqslant$ i78. Morbid anatomy. The anatomical changes given here are restricted to the case of Pearson and Ravenel. The animal was much emaciated. The mucous membrane of the small intestine was catarrhal and showed a small amount of erosion. All the organs were normal except the lungs. The most striking features on external examination was the extreme amount of emphysema. The lobules were separated from each other by 3 to $5 \mathrm{~mm}$. and even at some distance from the borders one conld see through these crevices by transmitted light. On the surface, the sub-pleural connective tissue was distended by large blebs. Upon palpation the lung crackled and numerous hard nodules could be felt. On section numer- 
ous dark red nodules appeared in the surrounding normal tisstre. In each lung there were from fifty to sixty of these nodules, from 5 to $12 \mathrm{~mm}$. in diameter. Most of them were dark red and closely resembled partially organized blood clots. However, on crushing a portion in glycerin between two slides and examining under the microscope, they were found to be made up almost entirely of a felted network of mycelial threads. Between these large nodules there were numberless smaller areas of mucl the same color, I to $2 \mathrm{~mm}$. in diameter, not perceptible to the touch as nodules but which were of the same character and were no doubt foci of recent origin. These were seen especially well in portions of the lung which were preserved by Pick's method, the slight bleeching of the tissue bringing them into relief. On opening some of the interlobular emphysematous spaces, small, whitish, mouldy looking patches were noticed which bordered the cavity. Scrapings of these patches were made up entirely of perfect fruit hyphae, with myriads of spores. The diagnosis of a mould mycosis was in this way made at once and confirmed by cultures and examination of sections. Cultures were made on glycerinated potato, bouillon and plain agar, by opening a nodule with sterile instruments and tearing out a small portion of the center, which was transferred to the culture tubes and placed in an incubator at $39^{\circ} \mathrm{C}$. Abundant growth was obtained on the potato by the end of thirty-six hours, white at first but soon changing to a yellowish and later to a dark green. The growth in the bouillon and agar was slow. Plates and flasks of bread paste were made, and these with potato were employed for all subsequent cultures. The formation of the fruit hyphae was studied and the spores measured a number of times, being from 2.5 to $3.5 \mu$ in diameter. By these means the culture was identified as the aspergillus fumigatus. The experiments on other animals were limited to the inoculation of one rabbit, into the aural vein of which one-half cubic centimeter of a suspension of the spores was injected. The animal died in forty-four hours and from the liver and kidneys cultures were recovered. All of the organs were examined in sections, but the mycelium was detected in two only. 
Microscopic examination. The nodules were hardened and embedded some in collodion and some in paraffin. Various staining methods were tried, hematoxylin and eosin, carbolthionine, Gram and lithium carmine with Weigerts fibrin stain. Good results were obtained with all, but the carmine and Weigert gave the nost beautiful picture and by this method the fungus was most perfectly demonstrated, the spores and mycelium taking on a deep purple color. The histology was studied largely in sections stained with hematoxylin and eosin. The bronchial epithelium was normal in places, but, for the most part, the columnar cells have been replaced by a sort of membrane, which appears to be made up almost entirely of a felt-work of mycelial, threads. From this membrane hyphæ grow out into the lumen of the bronchus, and here, owing no doubt to the supply of air, fruit hyphre arise, with perfect sterigmata and spores. There is no cellular nor other exudate and very little debris. The under surface of this membrane is of looser texture, and contains some cellular infiltration made up of round cells, leucocytes, proliferated connective tissue cells and red blood corpuscles. The adjacent structures are closely filled with a cellular infiltration with a quantity of mycelium of the same description, this extending to the neighboring alveoli which under low power appear to have preserved their outline but with greater amplification are seen to have lost all their normal structure, showing clumps of homogeneous, irregular masses which stain faintly with eosin and are probably of connective tissue origin.

In these areas the mycelium followed the alveolar wall as a trellis, the tissue seeming to afford no obstacle to its advance. Within the alveoli is a finely granular debris, with some coarser particles, probably the remains of cells. In sections stained with carbol-thionine large numbers of mast cells are seen in the alveolar walls. Bordering these degenerated areas are alveoli which have retained their normal structure and are filled with a network of fibrin holding in its meshes a few cells. In other parts of the sections are areas resembling those just described, but in which all anatomical land marks have been destroyed, so that it is impossible to tell whether or not the spaces seen are bronchi. 
Some sections show a widespread interstitial and alveolar hemorrhage, the blood showing a considerable increase in the number of leucocytes. The capillaries are congested and areas of oedema with thickening of the alveolar walls are not uncollmmon.

There is peribronchitis and arthritis, while in some sections arterial thrombosis is seen, the thrombus being penetrated by mycelium, though no fruit is found. Areas are also found in which the alveoli are filled with a cellular exudate producing consolidation and thickening of the alveolar walls.

Emphysema, both interstitial and vesicular, is marked and often extreme. Around the borders of the interstitial cavities is a distinct zone made up of red blood cells, leucocytes and hemogeneous material, which is yellowish both in fresh as well as in stained sections. These areas contain very little mycelium. All sections show a small amount of anthracosis. The appearance of sections varies in different nodules as they are taken further and further away from the center. In general the fungus is thickest at the center, so thick in many instances that the lung tissue is hidden entirely and grows less as we go outwards. The tissue changes noted, take place in a zone beyond the greatest growth of the fungus. In other nodules the fungus is evenly distributed throughout following the alveolar walls. In these the tissue changes are slight. At times the fungus grows in dense, brush like clusters, closely resembling actinomycosis under low amplification. This form is considered to show a marked reaction and resistance on the part of the animal and a lowered vitality in the fungus. When found it indicates that the aspergillosis is a primary and not a secondary or terminal affection. No giant cells were found in any section.

Fruit formation was not observed in the substance of the tissues at any time. It was observed most frequently in bronchi, which were for the most part denuded of their epithelium and next in emphysematous cavities, where it could be detected in clusters by the naked eye. Fruit was found in sections, in spaces the nature of which it was impossible to determine accurately. Wherever the formation of fruit was 


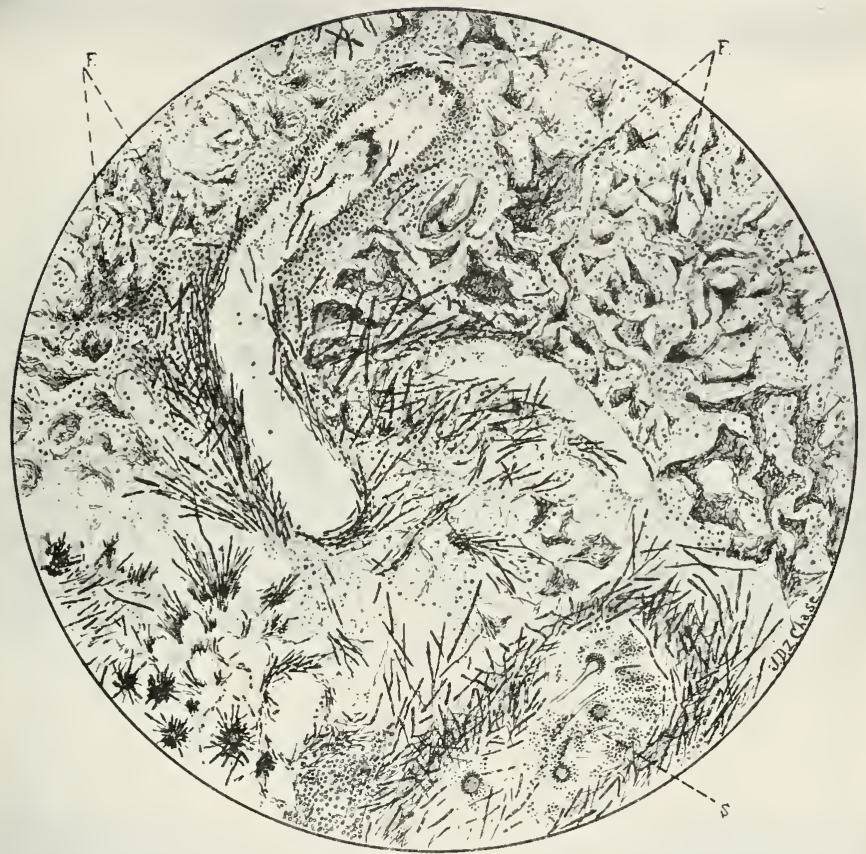

Composite drawing of section of Lung through nodule of aspergillus origin. F. fibrin in alveoli. S. fruit hyphae and spores of fungus. (Ravenel.) 


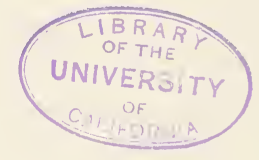


seen, there were innumerable free spores as well as those still attached to the sterigmata, but in no case were spores detected in the substance of the tissues.

In many sections, especially those from near the center of the nodules, the mass of mycelium was so dense that the structure of the tissues was obscured. Besides the dense growths resembling actinomycosis already described, other brush like clusters not unlike them were frequently seen. These differ from the former in being somewhat less compact, and that from their periphery numerous hyphae run out into the surrounding tissues, whereas in the actinomycotic form the masses are sharply defined and only here and there a few threads grow out beyond the cluster. Their appearance suggests that they may be actinomycotic forms which liave finally overcome the resistance of the tissues. Emphysema is less marked in the neighborhood of the latter.

The cow was examined carefully for the lesions of tuberculosis and in one lung some four or five caseous and calcareous nodules I cm. in diameter were found in which tubercle bacteria were demonstrated, but no mycelium could be detected. In no part of the lung was there coexistence of the two infections.

The fact that the animal did not react to tuberculin, though tuberculosis was present, is noteworthy and suggests that the mould infection may interfere with the test. It adds a possible source of error in the tuberculin test to be guarded against.

\section{REFERENCES.}

I. ARWINE AND LAMB. A fifth case of "fungous foot" in America. The Am. Journ. of Med. Sciences. Oct., I899.

2. Dinwiddie. On the toxic properties of moulds. Bulletin No. 10, Arkansas Agric. Exp. Sta. May, ISg6.

3. FLEXNER. Pseudo-tuberculosis Hominis Streptothricha. The Journal of Experimental Medicine. Vol. III. (1Sg8) (Bibliography).

4. OpHÜLS AND MofFitr. A new pathogenic mould. The Philadelphia Med. Journal. June 30, 1900.

5. PEARSON AND RAVEnEl. A case of pneumonomycosis due to the Aspergillus funigatus. The University Medical Magazine. Aug., 1900. The. Vet. Journal. New Series. Vol. II. (I900) p. 229.

6. KinON. L'Étude sur l'aspergillose chez les animaux et chez 1'homme. 1897 .

7. Weis. Four pathogenic Tourulæ (Blastomycetes). The Jour. of Mled. Research. Vol. VII. (1902) p. 280. 
CHAP'TER VI.

\section{DISEASES CAUSED BY PROTOZOA.}

TEXAS OR SOLTHERN CATTLE FEVER.

Synonyms. "Red water" ; Mexican fever ; Spanish fever ; Splenic fever, " Bloody Murrain'”.

$\leqslant$ I79. Characterization. Texas fever is an infectious blood disease of cattle, characterized by rise of temperature, hemoglobinuria, destruction of the red blood corpuscles and the presence in the blood of a protozoan parasite which is transmitted from animal to animal by means of the cattle tick.

It is believed to be identical with the hemoglobinuria in Roumania, Tick fever in Australia, and "La Tristeza" in South America. It has been named malaria des bovides by Celli and Sentori and Malaria Bovine by Lignières. Although it differs in many ways from human malaria, the analogy is so close respecting the specific cause, wide distribution and means of transmission that bovine malaria seems to be a very suitable name for this affection. At least it has the advantage of not stigmatizing any locality.

The pectuliar and interesting feature of this affection is the fact that cattle raised in the infested districts become immunized so that they do not suffer from the disease but they carry its specific organism in their blood. When imported into non-infested districts, they transmit the virus, by means of the cattle tick, to susceptible animals, but remain themselves perfectly well.

S I80. History. There is little knowledge concerning the early history of this disease. With the development of commerce, however, the shipping and interchange of animals gradually came into prominence and with it all, this disease 
which had long been known in certain localities was more widely scattered and finally it came to be an important barrier to the cattle traffic. In I 868 , the disease seems to have made its first important impression upon the Anerican people. In June of that year, Texas cattle were shipped up the Mississippi river to Cairo and thence by rail into the states of Illinois and Indiana where they cansed during the summer enormous losses from this disease. Cattle from these states shipped east brought the disease with them. The cattle commissioners of New York and the Board of Health of New York City endearored to check the importation of such cattle. The disease was carefully investigated at that time but nothing beyond a very accurate description of the gross lesions was obtained. Later Dr. D. E. Salmon determined the boundary line between the non-infected and the permanently infected districts, or what is now known as the Texas Ferer line (See Plate VII). In I889, the Bureau of Animal Industry undertook a systematic investigation into the nature of this disease, which resulted in the same year in the discorery of its specific cause by Dr. Theobald Smith and later the demonstration of the fact that the disease is transmitted from southern to northern cattle through the medium of the cattle tick. Prior to this (I888) $\mathrm{V}$. Babes had found an intraglobular parasite in the blood of cattle suffering from an epizoötic disease (henoglobinuria) in Roumania. While at first these diseases were thought to be different in their etiology they are now believed to be identical. Recently a nuinber of investigations have been made by the Burean of Animal Industry, the State Experiment Station and State Board of Agriculture of Missouri, the Louisiana Experiment Station and by the Queensland Gorernment, Australia, for the purpose of obtaining a practical method for the production of immunity against Texas fever in susceptible cattle. Although the results thus far obtained are promising aa they are often successful, the methods must still be considered in the experimental stage.

S181. Geographical distribution. In the United States the distribution of Texas ferer corresponds with that of the cattle tick (Boophilus bovis). This includes, with possi- 
bly a few small exceptional areas, that portion of the country south of the "Texas fever line" which is shown by a red line on the accompanying map but which is changing constantly by virtue of the spread or elimination of the tick. It has been identified with the tick fever of Australia which has become a source of great loss to the cattle industry of that country. Lignières has identified the disease in South America (Argentine Republic). It is also reported to be identical with a malady affecting cattle along the Danube River, in the Balkan provinces and in Southern Africa. It is restricted, however, to those countries where the climate is not sufficiently severe to destroy the cattle tick during the winter season and where the animals are constantly infested. Cattle (genus Bos) are the only animals which suffer from it.

\$ 182. Etiology. Texas fever is caused by a microörganism belonging to the protozoa and named by Smith, the discoverer, Pyrosoma bigeminum.* It is generally recognized that Piroplasma is the proper generic term and it is used by writers on the protozoa.

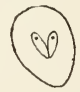

Fig. 54. Piroplasma bigeminum in red blood
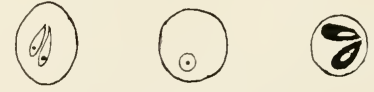
corpuscles.

If Texas fever in America, and hemoglobinuria in Roumania are identical, Babes was the first to call attention to the existence of this intra-globular parasite. It seems that Dr. Stiles in I 867 observed this organism but failed to recognize its significance. It is found in the blood in cases of Texas fever and it also exists in the blood of immune animals in the tick infested districts. The life history of this parasite is not determined. In the blood of the diseased animal they appear in the unstained, fresh preparation as minute or larger bright bodies which may be from 0.5 to $4.0 \mu$ in diameter according to the form of the disease. In the acute types of the

*The genus of this parasite has been changed to Piroplasma by Patton and Apiosoma by Wandelleck, to Amcbosporidies by Bonome, and Proteus virulentissimus by Perroncito. Starcovici has named the organism described by Babes as Hematococcus, Babesia bigeminum bovis. 
disease certain of the red corpuscles contain pale or brighter

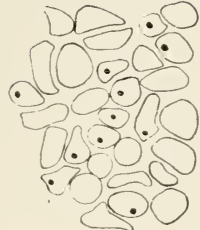

FIG. 55. Coccus form of Piroplasma bigeminum. pyriform shaped bodies. One end of each body is broad and rounded, the other tapering and pointed. Usually there are two of these bodies, both of the same size in a corpuscle. More rarely there is but one, although four are occasionally observed (Fig. 57). When two are present the tapering ends approach each other and usually they are joined while the other ends may point in any direction. Several forms have been noted varying from a round to a pyramidal outline. The small and often the larger bodies have been observed to change their position within the red corpuscle. Smith has noted that the amoeboid bodies observed were apparently single within the corpuscle. In dried and heated cover-glass preparations stained with alkaline methylene blue, these parasites are colored distinctly. They are also stained with carbol fuchsin and with hemotoxylin. As a rule they stain more deeply in preparations made for internal organs than they do in those from the living blood.

In the capillaries of the congested organs, the blood corpuscles contain many more parasites. Smith has noted in one case from 2 to 3 per cent of infected corpuscles in the circulating blood but in cover-glass preparations made at the autopsy quite different results, viz.: in the skeletal muscles, blood of the right heart, and blood from the bone marrow (sixth rib) very few infected corpus- (Smith).

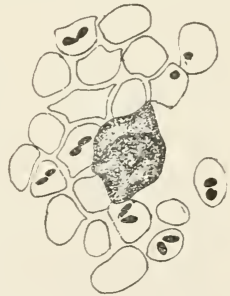

FIG. 56. Blood from kidney showing parasites of Texas fever. cles; in the blood from the left heart and lung tissue from 2 to 3 per cent of infected corpuscles; in the spleen 5 per cent; in the liver and kidney tissue from Io to 20 per cent; and the hyperemic fringes of the omentum and the heart muscle 50 per cent of the corpuscles were infected. In other cases the 


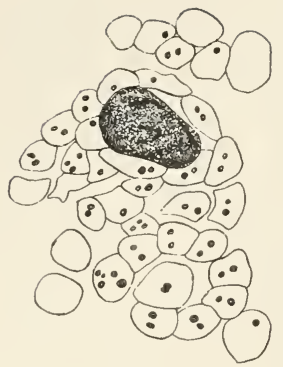

Fig. 57. Cover-glass pre. paration from kidney. Cor- in diameter at the periphery of the puscles showing piroplas-corpuscle. In stained (alkaline mema, coccus form. (Smith). thylene blue) preparations, the parasites appear as round coccus-like bodies from 0.2 to $0.5 \mu$ in diameter. They are situated within the corpuscle on its border. As a rule only one is found in a corpuscle. Sometimes a division was evident separating the parasite into two parts. They must be differentiated from somewhat similarly looking bright bodies which are seen in the corpuscles of healthy blood during different seasons of the year.

Concerning the life history of this parasite, Smith considered the intraglobular stage hypothetically the swarming stage, which precedes the peripheral coccus-like bodies and the pyriform and spindle shaped bodies which develop from the divided coccus-like peripheral forms. The free bodies are the parasites set free after they have reached the preceding stage by disintegration of the infected corpus-

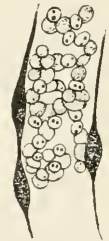

FIG. 5 S. Blood in capillaryof heart showing $P i$ roplasma. (Smith). cles. They are most commonly found in the kidney. The reproductive stage has not been recognized.

\$82. Infection. Although practical stockmen had long looked upon the tick as the source of infection it remained for Smith and Kilborne to experimentally demonstrate that so far 
as known the cattle tick (Boöphilus bovis)* is the sole carrier of the parasite. It was pointed out by them that when southern

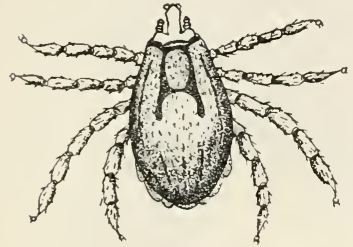

FIG. 59. Sexually mature male tick after the last moult, dorsal view. (Smith.) cattle were freed from ticks they would not when kept together in small enclosures transfer the disease to susceptible animals, but that when susceptible cattle became infested with the ticks either by grazing in infested pastures or by having placed upon them young ticks hatched in the laboratory the disease appeared. $t$

The infection of Northern cattle with Texas fever by Southern animals consists therefore in first infesting them with the cattle tick. The number of ticks necessary to carry the disease is small so that frequently they will not be observed unless the sick animals are carefully examined. The life cycle of the tick will, therefore, explain the variation in the time elapsing between the exposure of northern to southern cattle and the appearance of the disease. Starting with tick infested aninals placed with native cattle in a northern pasture the adult female ticks drop to the ground almost daily so that the following life cycle may be assumed to begin at once.

*This tick was first described by C. V. Riley in IS6S as Ixodes bovis. Later, Cooper Curtice investigated this parasite (Biology of the Cattle Tick, Journ. Comp. MIed. and Veterinary Archives, July, iSgi, Jan., IS92 ) and gave it the generic name of Boophilus (ox loving). This seems to be the only species of cattle tick which transmits the parasite of Texas fever.

$\rightarrow$ It is interesting to add the results of an experiment conducted by Dr. Cooper Curtice in the Bureau of Animal Industry which shows the necessity of the tick in inoculating cattle. In a tick infested district in the South, a field was cleared of ticks by fencing and keeping cattle off for a year. Susceptible northern cattle were transported to and placed in this field where they thrived for a season. The second year they were placed in a second cleared pasture where they kept well for another year. The third year they were placed in a tick infested pasture where they died promptly of Texas fever. 


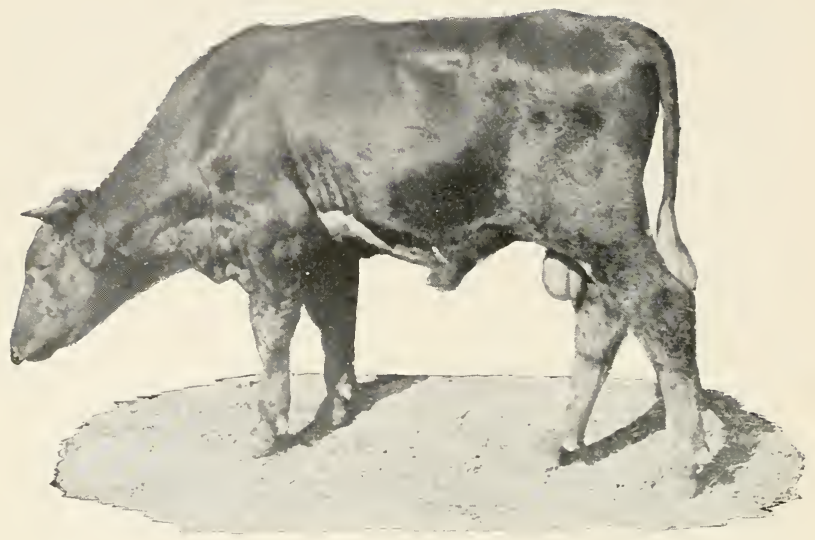

FIG. 6o. Photograph of animal sick with Texas fever. (Photographed by Connazuay).

I. Adult ticks drop to the ground in from 1 to 3 days after the infested cattle are placed in the field.

2. Adult ticks lay their eggs in about 7 days after dropping to the ground.

3. Eiggs are hatched in about 20 days after they are laid.

4. Young ticks crawl upon cattle from I to several days after they are hatched.

5. In about 10 days from the time the young ticks crawl upon the susceptible cattle the rise of temperature appears.

The length of time that must elapse (period of incubation) from the exposure of susceptible cattle to the development of the disease depends on whether or not the whole life cycle of the tick must be passed or part of it has already gone by. If susceptible animals are placed in a pasture where the young ticks are just ready to crawl upon them the infection of the cattle is accomplished at once and the high fever appears in about ten days, practically the minimum time. It has been experimentally demonstrated that the young ticks are able to 
travel for a considerable distance in a pasture. In pasures where tick infested cattle are grazing, young ticks are very liable to be on the ground continuously. In est i mat $\mathrm{i} n \mathrm{~g}$ the time to elapse after the exposure to the tick infested field

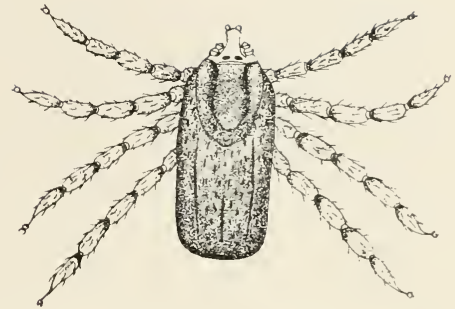

Fig. 6r. Sexually mature female after the last moult, dorsal view. (Smith.)

before the disease will appear, it is necessary, therefore, to determine the exact stage in the life circle of the ticks at the time when the animals come in contact with them.

Small quantities of the blood from immunized cattle in the tick infested district, when injected into susceptible animals either intravenously or beneath the skin, will produce the disease. While this mode of infection rarely if ever occurs in the natural order of events, it may happen that in case of certain operations bits of blood may be carried directly from a southern to a northern animal thus inoculating the latter with the disease.
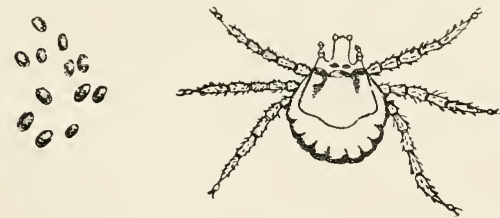

In the fall of 1898 two cases occurred in the practice of Dr. Ambler of Chatham, N. Y. The owner had his animals dehorned

FIG 62. Eggs and young tick, just hatched. in December and (Smith.) soon afterward two fatal cases of Texas fever developed. The Piroplasma and the characteristic lesions were present. Inquiry revealed the interesting fact that the two animals which sickened and died were dehorned immediately after two imported Southeru cattle. The owner was not aware of the fact at the time that these were 
Southern bred cattle, as he had bought them of a dealer in Vermont. More recently another case of this disease produced in the same way has been reported.

$\$ 183$. Symptoms. In the acute type of the disease which occurs during the hot summer months, the onset is sudden and usually all animals exposed to the same infection together come down at the same time. The first indication of the disease is a rise of temperature, at first higher in the afternoon than morning, but this oscillation is minimised later in the course of the disease when the temperature remains high. The temperature rarely rises above $107^{\circ} \mathrm{F}$. With a clinical thermometer the temperature can be detected two or more days before there are other symptoms. The respiration may rise to between 60 and 100 and the pulse may range between 80 and I IO per minute. Late in the disease there may be hemoglobinuria. Smith and Kilborne found it in 33 out of +6 fatal cases in which urine was found in the bladder. The passing of the colored urine before death was noted in but four of their cases. In one of these which showed hemoglobinuria four days before death, the urine in the bladder was clear at post mortem. As this condition seems to depend upon the rapidity of the destruction of the red blood corpuscles, a slow disintegration may enable other organs to dispose of the coloring matter which in a rapid destruction of the blood much of it way be thrown into the urine. The urine contains small quantities of albumin. At first the specific gravity may be high but later it ranges from roro to IO2O and fails to effervesce with acids. The color varies according to the quantity of hemoglobin. As a rule there is marked constipation during the high fever. There is loss of appetite and usually cessation of rumination with the high fever. The blood is thin and pale. The high temperature, hemoglobinuria and thinness of the blood are quite diagnostic symptoms of the acute type.

The course of the disease may vary, but the continuous high temperature does not usually last for more than ten days. Death often intervenes in from five to eight days. In the mild, nonfatal or chronic type which was first pointed out by Smith and Kilborne and which occurs in the late summer or 
autumn, the general symptoms are similar to those of the acute type except they are not so severe and are prolonged for a greater length of time. The parasite is of the spherical or coccus form. The general symptonis are not manifested unless the temperature goes above $\mathrm{IO}^{\circ} \mathrm{F}$. Hemoglobinuria is not observed in this type. Cattle which have passed through an acute type owing to the heated season may have a relapse in the form of a mild type in the fall. The essential difference between the two types is found in the different stages of the parasite circulating in the blood. Unless the temperature is taken and the blood carefully examined, mild types of Texas fever would be either overlooked or mistaken for any one of a variety of disorders common among cattle.

s84. Morbid anatomy. Cattle which die of Texas fever undergo post-mortem changes very rapidly. For this reason the description of lesions made some hours after death may be misleading.

Externally the animal presents nothing abnormal or characteristic of the disease. Rarely dried bits of blood may be found and also some small slightly elevated areas of a bluish color. The skin between the thighs, upon and about the udder and possibly elsewhere may have cattle ticks attached. It is inportant under ordinary circumstances to look for this parasite. The subcutaneous tissue may be more or less yellow in color. Oedema of the subcutis over the ventral portion of the body has been observed. The nuscles are usually normal in appearance although frequently they are pale.

Very sliglit if any lesions have been recorded as occurring in the central nervous system and lungs. Blood extravasations usually occur beneath the skin and endocardium especially of the left ventricles. On the external surface the petechiae occur for the greater part along the intraventricular groove near the base. The capillaries of the heart muscle are packed with corpuscles. Parenchymatous and fatty degeneration of the muscular fibers sometimes exist. The right ventricle is distended with blood either fluid or clotted and the left one firmly contracted. 
In the abdominal cavity there are frequently oedematous areas about the kidneys and in the portal regions between the duodenum and liver. The omentum is often sprinkled with peculiar hyperemic patches consisting of delicate shreds of vascular tissue. This condition, however, is not characteristic of 'Texas fever.

Usually the most conspicuous changes are in the spleen. This organ is much enlarged and increased from two to four times its normal weight. The normal markings, Malpighian bodies and trabeculae, are hidden in the dark browish-red, glistening pulp which distends the capsule. The pulp may be firm or it may be in a semi-fluid condition so that it oozes out if the surface is cut. The enlargement and color of the spleen are due to an engorgenent of red blood cells. There may also be present a greater or less number of large cells containing granules, red corpuscles or clumps of yellowish pigment. Free pigment is much more abundant than it is in healthy spleens.

The liver is extensively affected. It is enlarged, congested, edges rounded, the bile ducts more or less distended and the parenchyma is usually in a state of fatty degeneration. The color is paler than normal and usually the surface is somewhat mottled. On section the color of the cut surface is brownisli-yellow or it may be mottled like the surface. The mottling is due to a discoloration due to degeneration of a zone bordering the intralobular vein. This zone varies in width and its peculiar color seems to be due to a tendency to necrosis. It is characterized by parenchymatous degeneration and the loss of the nucleus. It may involve a third or more of the lobule. This portion stains very feebly or not at all. The explanation for the necrosis of the liver cells is suggested by Smitl as being due to the plugging up of the ultimate bile canals with solid bile which may interfere in some way with the nutrition of the adjacent liver cells. The bile stasis he considers as a result of the breaking up in the capillaries of the liver of enormous numbers of infected corpuscles. This results in an abnormal fluid containing an excess of solids which the bile ducts are tunable to carry away. When 
examined in fresh condition or in sections of tissue fixed in Mïller's fluid the engorgenent of the bile canaliculi is seen. The bile stasis may occur orer a portion or a whole of the lobule. The gall bladder contains usually an abnornal quantity of changed bile. It is thick and often semi-solid in consistence holding in suspension many flocculi. It imparts a deep yellowish tinge to all articles coming in contact with it. Owing to the mucus which is present it is quite viscid.

The changes in the kidneys vary. If death occurs early they are usually enlarged and uniformly darker than normal throughout. The capillaries are distended with red corpuscles. Parenchymatous and fatty degeneration are not common although occasionally present in the epithelium of the tubules. The pelvis is often sprinkled with ecchymoses.

The bladder may contain ecchymoses.

It is important to note that throughout the kidneys, liver and spleen pigment may be more or less abundant.

In the digestive tract the lesions of this disease consist largely of congestion of the mucosa especially in lines corresponding to the summits of the folds of the nucous membrane. It is more marked in the caecum and rectum than in the colon. The caecum and less often the rectum contain dry, hard fecal balls. In some cases in this system lesions are not observable. In the older writings much emphasis is placed on certain lesions, mostly nodular or abrasions, in the digestive tract. Smith has shown, however, that most of these are due to animal parasites and have no relation to Texas fever.

As already stated Texas ferer is a disease of the blood and consequently it is in this fluid that the most constant and morbid changes occur. They are characterized by the blood becoming thin and watery with a destruction of the red blood corpuscles. In some of the cases the loss of corpuscles is rapid and continuous until death or convalescence, while in others there is a marked oscillation between destruction and regeneration. In some animals the loss is not continuous, but the course of the disease is marked by periods of rapid blood destruction and periods of rest, or, as it were, where the blood destruction was holding its own. These points are best illus- 
trated from actual cases three of which are taken from the report by Smith and Kilborne.

\begin{tabular}{|c|c|c|c|c|}
\hline (No. I 29.) & $(\mathrm{N}$ & I42.) & & . 56.$)$ \\
\hline $\begin{array}{c}\text { No. of } \\
\text { Corpuscles. }\end{array}$ & Date. & $\begin{array}{l}\text { No. of } \\
\text { Corpuscles. }\end{array}$ & Date. & $\begin{array}{l}\text { No. of } \\
\text { Corpuscles, }\end{array}$ \\
\hline $\begin{array}{rr}\text { Aug. I1, } & 6, \text { I 23,000 } \\
\text { I3 } & 7,171,000 \\
\text { I6, } & 5,370,000 \\
27, & 3,210,000 \\
29, & 1,675,000 \\
30, & \text { Died } 8 \text { P. M. } \\
\text { First high A. M. temp. } \\
\text { Aug. } 24 .\end{array}$ & 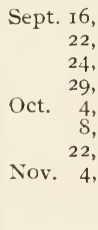 & $\begin{array}{l}6,890,000 \\
5,430,000 \\
4,562,000 \\
5,274,500 \\
3,902,000 \\
5,953,000 \\
4,333,000 \\
5,586,000\end{array}$ & $\begin{array}{rr}\text { Sept. } 20 \\
22 \\
29 \\
\text { Oct. } 9 \\
22 \\
25 \\
30 \\
\text { Nov. } 6 \\
8 \\
13\end{array}$ & $\begin{array}{l}6,844,000 \\
5,640,000 \\
5,307,000 \\
5,436,000 \\
4,666,000 \\
2,754,000 \\
2,720,000 \\
2,344,000 \\
1,984,000 \\
1,1 S_{3}, 000\end{array}$ \\
\hline
\end{tabular}

As evidence of the diminution of the number of corpuscles within the body these authors point (1) to the loss of hemoglobin through the kidneys, (2) the over production of bile which is abnormal in the abundance of pigment and (3) to the actual observation of their destruction by the micro-parasite under the microscope.

The regeneration of blood corpuscles is indicated perhaps by the count, but more surely by the form of the corpuscles themselves. The abnormal forms are the very large corpuscles, "punctate" forms and lastly the diffuse stained or "tinted" forms and the hematoblasts. The first of these may appear when the blood count reaches $3,000,000$ and the other forms when it is still lower. These various forms, however, are probably embryonic or immature corpuscles, which are forced prematurely into the circulation by the blood producing organs in trying to overcome the rapid destruction of corpuscles by the parasite. The action of the disease upon the leucocytes or the defensive activities of the white corpuscles in combating the parasite of Texas fever have not been determined. In the work thus far performed and reported, they have received little attention. Suffice it to say that they have not been in evidence in this conflict and probably take little or no part in the morbid changes of Texas fever. 


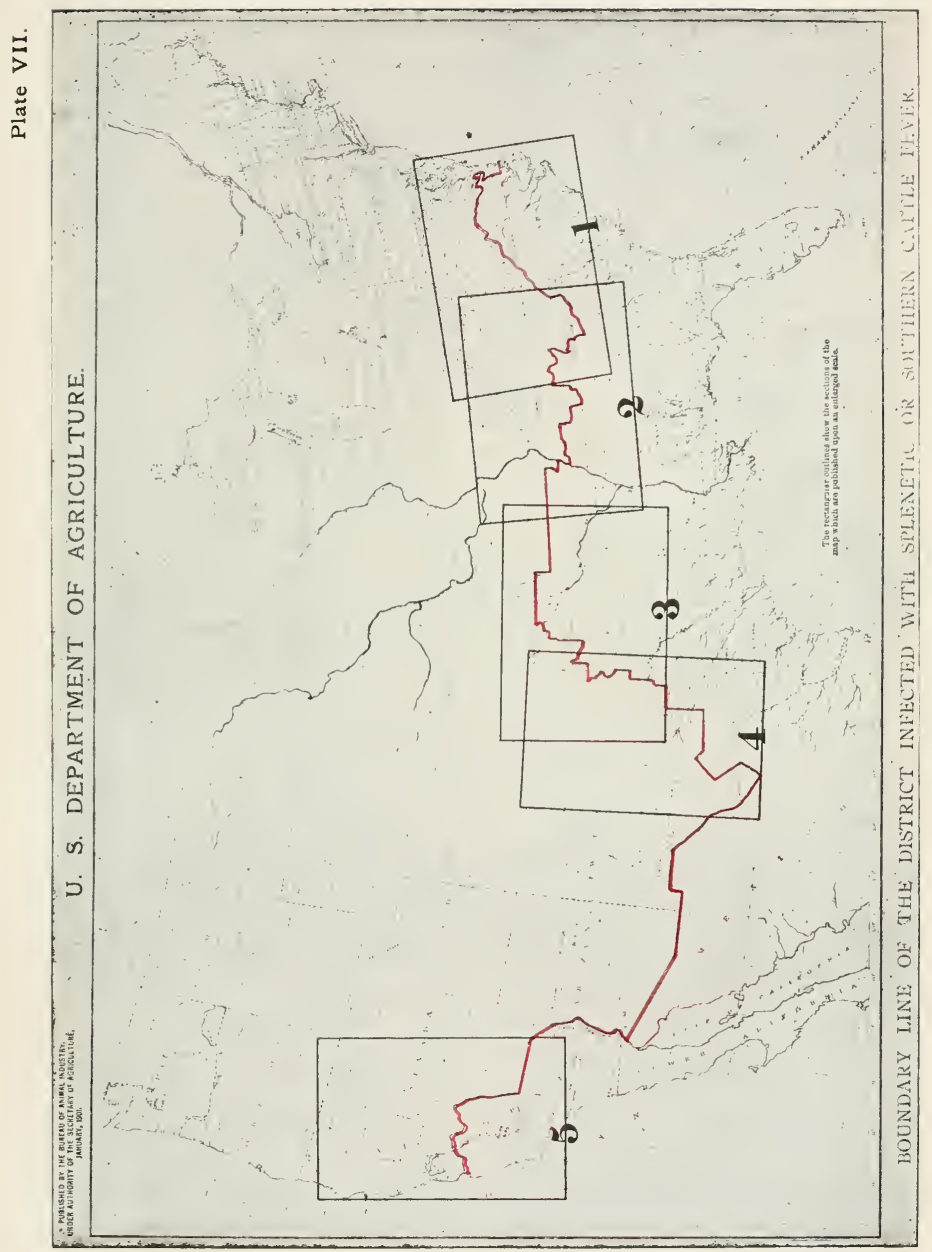


UTIVERE THSTTY
OF

URENIA 
$\$$ 185. Differential diagnosis. Texas fever is easily differentiated from other disorders or infectious diseases by the presence of its specific parasite.

In the absence of a suitable microscope the differentiation can in most cases be made from the character of the lesions, the history of the animals, the presence of the cattle tick and the course of the disease. The lesions are not simulated by any other disease of cattle, although the enlarged, dark spleen may suggest anthrax and in a hasty diagnosis the two may be confused. From the fact that all animals exposed together usually come down with the disease together poisoning may be suggested, but here again a study of the symptoms and lesions are sufficient to eliminate toxic disorders.

$\leqslant$ i 86 . Prevention. The discovery of the specific cause of Texas fever and of the cattle tick as the common means of its transmission has reduced the preventive nieasures to a direct warfare against the tick. The National Government has determined the territory in which the tick naturally exists and from which cattle, on account of the parasite, cannot be shipped to uninfected districts, except under certain very restricted conditions. (See regulations for transmission of cattle p. 618, Report of the Bureau of Animal Industry for I 898.) Likewise susceptible northern cattle cannot be transported to the infested districts (south of Texas fever line) unless they can be placed in fields that have been freed from ticks. The elimination of the disease depends therefore, upon the elimination of Boöphilus bovis. While this may be impossible in the territory of large ranches, there seems to be no reason why in the dairy districts of the South it cannot be accomplished.

$\$ 187$. Immunizing susceptible cattle. A number of investigations have been undertaken directed toward the development of methods for immunizing northern cattle against Texas fever in order to enable the shipment, especially of breeding stock, into the south. In 1895 , the writer in conjunction with Dr. E. C. Schroeder, began an immunizing experiment which was continued and reported by Dr. Schroeder in 1898 . 
Young aninials were selected and injected with blood taken directly from the jugular veins of southern animals. The injections were made in the fall and winter and in the spring the animals were placed in a highly infested field at Manchester, Va., where they remained for the summer. During this time they were under the immediate observation of Dr. Cooper Curtice who made a careful study of the blood, temperature and extent of tick invasion. The results of this experiment are shown in Dr. Schroeder's tabulation which is appended. The animals were again exposed the following season without the development of Texas fever.

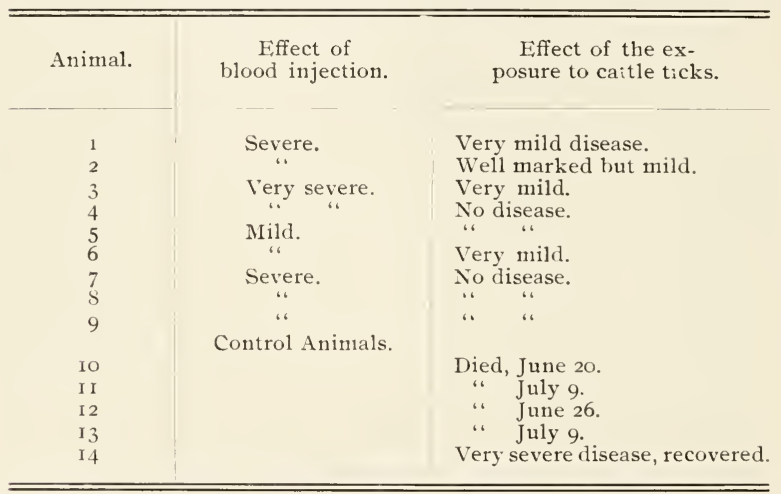

The inoculation disease appears in from eight to ten days after the injection of the blood. It lasts from one to two weeks. The symptoms are occasionally of a still shorter duration, but the altered condition of the blood persists in sone cases for a much longer period.

Dalrymple, Dodson and Morgan, of the Louisana Experiment Station, conducted experiments along this line. To show the results of their investigations, a somewhat condensed summary of their experiments is given.

Immunity against a fatal attack of Texas fever can be conferred on susceptible cattle by inoculation with the blood of 
a native Southern animal or one which has recently been rendered immune.

When used in five cubic centimeter doses, blood from a recently immunized animal gave a less virulent and less protracted form of the artificially produced fever than a similar amount from a native.

Two and one-half cubic centimeters of blood from a recently immunized animal gave satisfactory results on two imported Herford calves, aged eight and five months respectively. Two cubic centimeters of the same blood acted satisfactorily when injected in a one-month-old calf. Animals that had been allowed sufficient time to perfectly recover from the inoculation fever before being exposed on a tick-infested pasture suffered no apparent ill effects from the ticks. Animals exposed before convalescence was complete suffered a temporary relapse.

The Experiment Station of that state offers to immunize (free of charge) northern cattle, if they are shipped to the state, for its stock raisers

In Missouri, Connaway has immunized a few animals with the blood serum from immune (Southern) cattle. This method, however, needs further testing. In Mississippi, Robert has tried the serum for both prevention and treatment with somewhat similar results. In Virginia and Oklahoma the disease and its prevention have been studied. In Texas, Francis has conducted a number of immunizing experiments with most excellent results. He has pointed out the value of immunizing young ( 2 to 6 weeks old) calves by artificially infesting with ticks. In Australia the problem of immunizing cattle against the effects of the spreading of the cattle tick in order to save their stock from "tick fever' has become a matter of serious consideration.

The very limited knowledge of the life cycle of the parasite of Texas fever precludes a satisfactory explanation of the modus operandi in the production of immunity by these various procedures. On this point there is need for much extended investigation. 


\section{REFERENCES.}

I. BABEs. Die Aetiologie der seuchenhaften Haemoglobinurie des Rindes. I'trchow's Archiv. Jan. 1 S89.

2. Connaway. Texas fever or acclimation fever. Bulletin No. 37. Mo. State Board of Agriculture. 1897.

3. DALRYMPLE, MORGAN AND DoDSon. Texas or southern cattle fever. Bulletin 51 . Louisiana Agric. Exp. Station. ISgS.

4. Dinividde. Some Texas fever experiments. Bulletin No. 20. Ark. Agric. Exp. Station. 1893.

5. Francis and Connaway. Texas fever. Bulletin No. 35. Texas Agric. Expt. Station. 1899.

6. GaMgeE, DODGe, Bilings and Curtis. Diseases of Cattle in the United States. Report of the commissioner of agmculture, II ashington, D.C. 1871 .

7. Hunt and Colinins. Report on tick fever. Brisbane. Queensland, Australia, 1896.

S. Lewis. Texas fever. Bulletin No. 39. Oklahoma Agric. Expt. Station. I 899

9. Lignières. La "Tristeza" ou Malaria Bovine dans la République Argentine. Buenos Aires. 189o. (Full bibliograply).

10. Mayo. Texas Fever. Bulletin No.69. Kan. Agric. Exper. Station. 1897.

11. MCCULIOCH. The prevention of Texas fever and the amended laws controlling contagious diseases. Bulletin No. 10.7. I'irginia Agric. E.xpt. Station. I899.

I2. Niles. Splenetic or Texas cattle fever. Bulletin No. 6r. Virginia Agr. Expt. Station. 1896.

13. NôRGaARD. Dipping cattle for the destruction of ticks. Annual Report, Bureau of Animal Industry. 1895-6. p. Io9.

14. Paquin. Texas fever. Bulletin No. II. Mo. Agric. Exp. Station. 1890.

15. Salmon. Contagious diseases of animals. Special report No. 22. Washington, D. C.

I6. SAlmon. Report Commissioner of Agriculture. IS8I-2.

17. SAlmon. Annual Reports, Bureau of Animal Industry. 1884-5.

I8. Schroeder. Inoculation to produce immunity from Texas fever in Northern cattle. Ibid. ISg8. p. 273.

19. SiITH. Preliminary observations on the microorganism of Texas fever. The Medical News. Dec. 21, I889.

20. SMith AND Kilborne. Texas fever. Bulletin No. I, Bureau of Animal Industry. U. S. Dept. Agriculture. 1893.

21. Suith AND Kilborne. Anmual Report, Bureau of Animal Industry. IS9I-2. (Issued IS93).

22. STILES. Report New York State Board of Health. IS68.

INFECTIOUS ENTERO-HEPATITIS IN TURKEYS.

$\$$ 188. Characterization. This disease of turkey's is characterized by thickening of areas or of the entire walls of the cæca and areas of tissue degeneration or necrosis in the liver. It is popularly known as "black head." 
s r89. History. In the fall of 1893 , Prof. Samuel Cusliman of the Rhode Island State Experiment Station sent a few specimens of the diseased organs of turkeys which had died of "black head" to the Bureau of Animal Industry where they were carefully examined by Dr. Theobald Smith. In the summer of 1894 , Dr. Smith made a careful study of this disease at the Rhode Island Experiment Station. He found that it was caused by one of the protozoon (Amoeba meleagridis Smith) and he published a full description of the disease which, in accordance with the lesions, he designated Infections entero-hepatitis.

In 189.5, the disease was further investigated respecting the mode of transmission of the infecting protozoa. The results showed that it could be transmitted directly from diseased to healthy turkeys without the intervention of an intermediate host. These results were published in 1896 . Recently, Chester of the Delaware Agricultural Experiment Station has shown that a very similar disease attacks chickens. The place and the time of the first appearance of this disease are not clearly stated, but it seems that New England was the first to suffer from it.

S rgo. Geographical distribution. The available data bearing upon the geographical distribution of this disease indicate that it is widely distributed. The New England states, particularly Rhode Island, and certain districts in the middle and western states are affected. It has not yet been reported from the southern states. For want of statistics the amount of loss to the poultry industry occasioned by this disease can not be accurately estimated, but the fact that it has caused many farmers and poultry men in New England to discontinue the raising of turkeys shows that it is of much economic importance. It is stated in the report of the Rhode Island Experiment Station for 1894 that "the eradication of this disease would be worth hundreds of thousands of dollars to the eastern farmers alone." These heavy losses in the east, together with the accumulating evidence that the entire northern third of this country is sprinkled with infected districts from which the disease is spreading, indicate that this 
malady is of more than ordinary significance to those engaged in the turkey industry.

\$ I9I. Etiology. In 1895 , Smith described a microorganism belonging to the protozoa which he found to be associated directly with the disease process. He designated it Amoba mcleagridis. In those cases in which the disease was recent, or at its height, the parasites were very numerous in the affected tissues, while in those in which the disease process was far advanced and associated with degenerative or regenerative changes, the parasites were found with difficulty.

The most frequent appearance presented by the parasites is that of round homogeneous bodies with a sharply defined, single-contoured outline. Within these bodies and situated some what eccentrically is a group of very minute granules, probably representing a nuclear structure. They vary somewhat in size measuring from 8 to $10 \mu$ in diameter in of a turkey'scacum. (Smith).

some cases, from 12 to $14 \mu$ in others. In the fresh tissues they are distinctly larger than the parasites within the tissues, which have undergone the hardening process. The latter are from 6 to $10 \mu$ in diameter. This difference may be due to shrinkage, on the one hand, and on the other to a slight flattening of the bodies by pressure in the fresh preparations. These peculiar homogeneous bodies were found, as a rule, free in the crushed preparations, although occasionally giant cells were detected which contained a number of them. The cell nuclei of the giant cells are not visible in the fresh condition. 
Numerous coarse granules, less frequently fat globules, are embedded in its protoplasm.

Smith believed from the results of his investigations that the parasite lived in the interstices and lympl spaces of the tissue, but not within cells. This seems certainly true of the crecum. In the liver, the liver cells seem to become necrotic or else disappear so rapidly that it is impossible to determine just where the parasites begin to multiply. They do not live within the blood vessels, as they are not found within them excepting perhaps in a thrombosed ressel. They must, therefore, occupy the place of the liver cells. It is probable that they begin to multiply in the connective tissue adjoining the blood vessel and simply crowd out the liver cells, leaving the connective tissue stroma of the lobules in whose meshes they are found.

Their presence within giant cells is seen in almost every infected organ subject to examination. In teased preparations of the fresh tissues they are frequently found with remnants of the inclosing cells still attached. This intracellular condition is, however, a purely passive one so far as the parasite is concerned.

The microparasites within the tissues of the host seem to tend toward destruction. Both the death of the tissue itself and the repair seem to lead to the disappearance of the parasites. In most cases there may be seen in the same section a partial dissolution of some of the bodies, while others are still in good preservation. Evidently their life within the tissues is not very long.

A discharge of the microparasites which escape destruction probably takes place fron the walls of the crea, when these break down into the contents with which they are carried outward. A similar discharge may take place from the liver through the bile ducts into the intestine. Anpther way of dissemination is in the death of the diseased turkey and the dissolution of its body whereby the organisms are set free. The occurrence of amcbæ in intestinal affections of man was noticed by Lösch in 1875 . Since that time it has been the subject of many investigations. 
The points of analogy between the avian and the human disease are that in both there is an affection of the intestine (large intestine in man, cæca in turkeys) associated with liver disease due to amœbæ.

The intestinal wall in amœbic dysentery is greatly thickened, owing to an cedematous condition. It is also thickened in circumscribed areas and contains cavities filled with gelatinous-looking pus. The amœbæ vary much in size and contain vacuoles. They are found in variable numbers in the bottom of the ulcers and in the discharges. The large numbers of amcbre found in the intestinal contents led Councilman and Laffeur to infer an active multiplication therein. The presence of the parasites within the submucosa is described by these authors in one case only.

In the turkey, the parasites are always present in the connective tissue spaces of the mucous and submucous membrane. Their presence in the contents of the crecum is highly probable.

It differs from the Amaba dysenteria in being quite uniform in its appearance, varying but slightly in size (from 6 to I $\mu \mu$ in diameter) and in being free from vacuoles. Movements characterized as amceboid have not yet been demonstrated.

The liver affection in man appears usually as an abscess. In turkeys it appears as a variable number of foci in which the microparasites may be present in great abundance. The difference in the nature of the lesions must be largely attributed to the different reaction of the tissues of birds toward injuries.

I92. Symptoms. Diarrhoea is the symptom which sooner or later may be expected to appear. It probably occupies the most prominent place among the objective nanifestations. The disease of the caeca is presumably respcnsible for this. Diarrhoea occurs with at least one other disease of the caeca and with the presence of tapeworms. Emaciation is pronounced in very chronic cases but it is not constantly present. As it may accompany other wasting diseases, it can not be depended upon as an indication of this affection. As the disease progresses the turkeys become less actire, hang behind 
their flock or do not go out with it. Later the comb, wattles and even the skin of the head become dark colored, hence the popular name "'black-head."'

\$ I93. Morbid anatomy. Turkeys are attacked quite young. Snitl found a turkey about three weeks old in which the disease had already made considerable progress. It seems, moreover, as if the disease was contracted only by the young, because in the examination of turkeys of different ages the oldest show lesions of the longest standing; that is, such as had undergone the most extensive transformation. In general it may be said that the age of the turkey corresponds with the age of the disease process. The most serious and extensive destruction of tissue occurs in the turkeys in fall. In midsummer the disease is making most progress and the microparasites are present in gieater numbers. It is probable that the delicate tissues of the young are best adapted for the temporary liabitat and rapid multiplication of this parasite.*

The primary seat of the clisease is the caeca. From these the liver is secondarily invaded. Other organs have not been found to be attacked.

The lesions of the caeca are in substance a thickening of the wall, followed in most cases by a destruction of the epithelium and deeper portions of the mucons membrane. This destruction results in the outpouring of a coagulable fluid into the tube. The thickening of the wall may vary considerably in extent from case to case. It may be nniform over the greater portion of the tube or it may be limited to circumscribed patches. The commonest seat of these lesionsis near the blind end of the tube where it evidently starts and whence it spreads to other portions. Not infrequently only one caecum is diseased, the other remaining normal.

The affection of the caecum is due primarily to the multiplication of the microörganism which may take place chiefly either in the mucons nembrane, or in the submucous tissue, it may, though rarely, extend into the muscular coat. The

*In this regard it simply follows the rule observed by large numbers of parasites whose most destructive action is visited upon the young. 


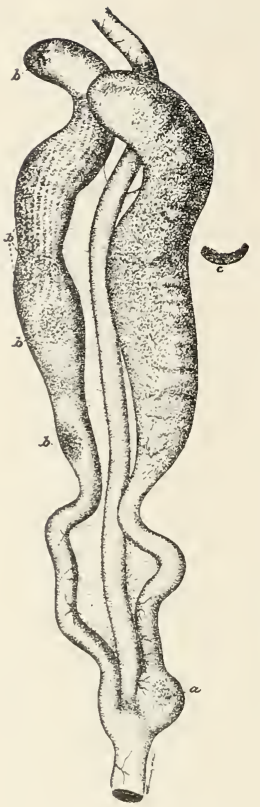

FIG. 64. Caeca; a and $b$ are diseased areas; c, section of thickened wall.

thickening of the wall is the result of several processes-the multiplication of the parasites, the increase of the normal tissue elements and later on the accumulation of masses of small cells and some giant cells.

In the early stages of the invasion, the adenoid tissue between the tubules and in the submucosa becomes greatly increased owing to the presence of large numbers of microparasites of round or slightly oval outline and from 6 to $10 \mu$ in dianseter which stimulate the proliferation of the tissue cells. Numerous mitoses have been seen in this stage. The parasites seem to occupy the meshes of the adenoid tissue either singly or in groups or nests. In these meshes they are soon enveloped in cells acting as phagocytes, so that the appearance of an intracellular habitat of the parasites is suggested.

The presence of the parasites in this reticulum probably stimulates also the accumulation of lympli cells within the spaces, in virtue of which the mucous membrane is thickened. In this early stage of invasion the epithelium, both of the tubules and of the surface, remains unaffected. The parasites do not invade the epithelium at any time.

As the disease progresses there is a continued increase in cellular elements of the mucous and submucous coats and a gradual invasion of the muscular coats. Here the bundles of fibers of the circular coat are thrust apart by masses of cells, so that this coat also becomes greatly thickened. The inflammation finally extends to the serous covering, where the blood vessels become greatly dilated and give the caecum a congested 
appearance. In cases of ordinary severity the wall of the caecum which is not more than 0.2 to 0.5 mm. thick normally becomes 2 to $3 \mathrm{~mm}$. thick.

With the progress of the disease the mucous membrane may be shed and a coagulable fluid poured out into the caecum. In some cases it appears in isolated masses, which adhere to certain spots of the mucous membrane. In others, this exudate fills the entire tube with a yellowish-white mass, built up in concentric layers consisting of a mixture of blood corpuscles, fibrin and small round cells in variable proportion.

In the further progress of the local disease it is not improbable that bacteria are also concerned. The exudate contains immense numbers of them and the denuded mucosa furnishes a favorable place of entry. It is otherwise difficult to explain the continued increase in thickness of the walls of the caecum after the mucous membrane has been shed. This coutinued increase in thickness is due to an extensive infiltration of small round cells and the presence of some giant cells. Parasites in this advanced stage are scarce and usually recognizable only as vacuole-like bodies within the giant cells.

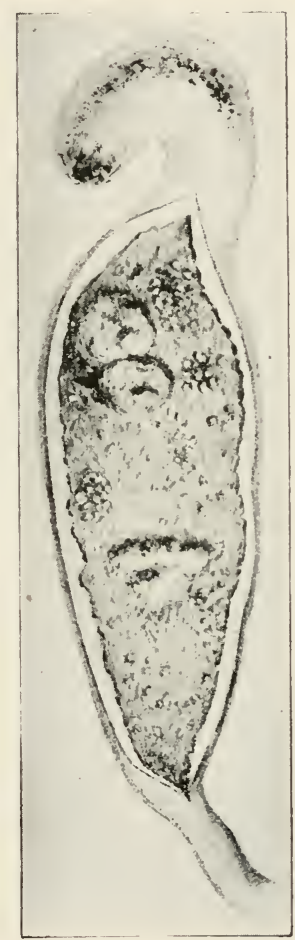

FIG. 65. Diseased caecum showing thickness of wall and ulcerated mucosa.

The thickening of the wall is associated in some cases with an extension of the inflammation to the contiguous wall of the intestine which becomes firmly attached to the caecum. 
Yellowish exudates are sometimes found outside of the diseased caecum on its serous covering and they bind it inextricably to the other caecum or to the intestine or attach it to the abdominal wall. In these stages, the microparasite is not found. It seems to have done its work by destroying the mucous membrane and to have left the field for miscellaneous bacteria.

Other portions of the digestive tract are not affected. The secondary lesions are found in the liver although in some cases they do not appear. The organ itself is enlarged to probably twice the normal size. Over the surface are distributed roundish, discolored spots, distinctly demarcated from the surround-

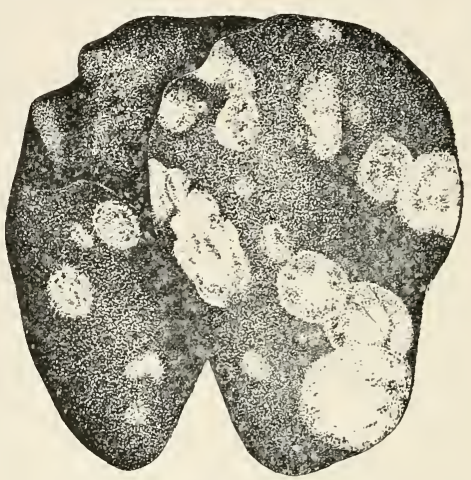

FIG. 66. Liver showing diseased foci.

ing tissue. These may be distributed uniformly over the whole surface of the liver or they may be limited in number to a few. They vary from 3 to $15 \mathrm{~mm}$. in diameter. Several types of these spots appear corresponding to different conditions of the diseased tissue. We have in the early, most active disease process sharply defined circular areas of a lemon yellow, of a neutral gray or of an ochre yellow color. The spot is not homogeneous in structure, but made up of a delicate network of grayish yellow, dead tissue.

In another class of spots there is a mottled brownish color which contrasts only slightly with the surrounding liver tissue by its darker color. It may contain a central yellow nuclens of dead tissue and a narrow outer border of the same character, or the border may be a dark brownish circular line. The entire spot has an indistinct appearance and is flattened or even 
slightly depressed below the surface. In some cases they are uniformly whitish and shade off somewhat gradually into the surrounding tissue. In sections of the affected organ it will be found that the surface spots represent masses of liver tissue in the same condition, the spots being simply the places where these diseased foci intersect the surface. Some are found deeply imbedded in the liver tissue, and therefore not visible on the surface. The lesion of the liver is thus represented by few or many foci of disease having in general a spherical form and appearing on the surface of the organ as round spots. Occasionally the lesions become more extensive and the death of large portions of liver tissue follows.

The changes in the liver are most easily explained by assuming that the microparasites are conveyed by the blood directly from the diseased creca into the liver and there deposited in different places, where they begin to nultiply and spread in all directions. In this way they form the spherical foci of disease which appear as circles on the surface of the liver. This theory is borne ont by the results of the nicroscopic examination.

In sections of hardened tissue from the liver in which the disease has but recently begun, the affected regions are invaded by large numbers of protozoa which occupy a kind of reticulum formed probably from the connective tissue stroma. The liver cells have partially or wholly disappeared from these foci. The parasites occupy the meshes of the tissue either singly or in groups. The reticulum is provided with a small number of nuclei, some of which are closely applied to and curved partly around the parasite. The blood vessels are usually much dilated and filled with red corpuscles.

The yellow masses observed with the naked eye in the surface spots are shown to be patches of an amorphous substance which take nuclear stains very feebly, the aniline colors not at all. It nuay be described as a coarse network in the meshes of which small cells, and very rarely parasites, are seen. This substance is assumed to be the result of coagulation necrosis of the liver cells by which they have lost their nuclei and have become fused into a formless mass. It is 
probable that the plugging of blood ressels in the liver by parasites carried from the cacum is the cause of the necrosis, since such plugs or thrombi are not uncommon in sections of the diseased spots.

With the appearance of the microparasites reactive changes begin at once which complicate the process. We have at the ontset an active multiplication of the microparasites which takes the place of the original liver tissue and a process of coagulation necrosis going on at the same time. Soon multinucleated (or giant) cells appear which not only take in the microparasites but which are also engaged in remoring dead tissue. At least their collection in groups around and within the necrotic areas leads to this assumption. Not infrequently they are grouped around what appears to be a plugged ressel or else they occupy the lumen of the ressel itself.

In still older cases the diseased areas are found more or less filled with small round cells which may have passed into the dead regions from the blood vessels. In all cases the latter are more or less enlarged and they seem to encroach upon the liver tissue, thus filling in part the void produced by the cell death and giving the surface of the liver a brownish, mottled appearance wherever the disease spots are. The processes of adrancing disease and necrosis or death of tissue on the one hand and of repair on the other seem to go on side by side, now one now the other predominating.

The results of the investigations thus far made indicate that the disease may follow several courses, viz.:

(I) After a certain period of disease regenerative processes begin which tend toward a permanent recovery.

(2) The disease may proceed so rapidly from the very start that the affected turkeys die early in life.

(3) The disease may come to a standstill but the amount of dead tissue in the caeca and liver may be so great as to faror the entrance of bacteria which are directly responsible for the death of the bird late in the summer or fall.

The description of the lesions of a turkey dead of this disease is appended. It is quoted from Smith's report. 
Turkey No. 17.-About 3 months old. Taken from a flock Angust $S$ becanse of lack of strength to keep up with the rest when driven. Indications of diarrhoea. Placed in a coop, where it died during the night. Examined next morning.

Slight ollor of decomposition. A few small warts on skin of neck. The varions organs were found normal, with the following exceptions:

Incosa of duodenum almost blackish, from intense injection and pigmentation of villi.

Both caeca diseased. The left is slightly distended. On serous aspect two yellowish spots, with markedly injected borders, corresponding to thickenings of the walls near the blind end of tube. The mucous surface of one is smooth; to the other an exudate is attached. Besides the thickening at these spots, the free half of this caecum is somewhat thickened uniformly.

The right caecum is very much distended over two-thirds of its length. From the serous surface local thickenings are recognizable, which have a yellowish, mottled appearance. The small intestine is firmly attached to one of these. The disease has, however, not invaded the wall of the latter. The border of these spots is intensely hyperæmic. When the caecum is slit open its width is three to four times that of the undistended tube, and the thickness of the wall varies from one-eighth to one-lialf of an inch, being not less than one-eighth of an inch over three-fourths of the entire length. When the brownish feces were washed away the increased local thickenings were found covered with firm exudates, usually attached in but one spot.

Sections were examined of that portion of the caecal walls which was very much thickened, and to which the contiguous small intestine was inseparately attached by the new growth.

The mucosa of the caecal portion had sloughed away, while that of the embedded small intestine was intact. The neoplastic tissue between caecum and intestine was fully $\mathrm{I} \mathrm{cm}$. (two-fiftlis inch) thick. Inasmuch as the infiltration probably followed the narrow mesentery between caecum and intestine the original boundary lines of the caecal wall are no longer recognizable. The muscular coat of the caecum may be traced for only a short distance into the neoplasm, when it disappears. Microparasites were not seen distinctly in the diseased tissue.

The liver is very much enlarged, and dotted everywhere with roundish spots of varying appearance. The majority are from 5 to $12 \mathrm{nmm}$. in diameter, round or slightly oval. The center of each is usually occupied by a group of yellowish dots and the circle is bounded by a narrow yellowish ring. The space of the circle is mottled brownish. Among these spots there are also circles of a completely yellowish color. On the convex surface of the left lobe there is a very firm, ring-like, yellowish mass, cutting like firm cheese.

In crushed preparations of fresh liver tissue from within the brownish circles many giant cells are seen. They consist of a meshwork of 
protoplasm of a ratler coarsely granular character inclosing spheres which appear homogeneous. The giant cells are up to $30 \mu$ in diameter.

Sections of liver tissue hardened in alcohol and in Foa's solution were also examined. The foci of disease contain necrotic areas in which are numerous giant cells each inclosing a number of microparasites. In some portions there is much cell infiltration in the interlobular tissue around the portal vessels. Among the cells the protozoa are recognizable.

$\$$ 194. Differential diagnosis. This disease is to be differentiated from certain local affections of the crecum not especially uncommon in turkeys. Zürn (Deutsche Zeit. f. Thiermed, X ( 1883 ). p. I89) has described a cæcal disease in water fowls and turkeys and Von Ratz has described a caecal disease in turkeys in which the liver lesions seem to be absent. The lesions in the liver and the presence of the nicroparasite, as previously described, are sufficient to differentiate this disease.

$\$$ I95. Prevention. The present knowledge of this disease shows that the parasite is transmitted directly from diseased to healthy turkeys. This suggests that the first precaution is to avoid the entrance of diseased or seemingly healthy turkeys from a diseased flock into a healthy one. The discovery of Chester indicates that a like precaution 1nust be taken with reference to fowls. If the disease exists the best though most radical method as suggested by Smith is the total destruction of the affected flock, thorough disinfection and the introduction of healthy turkeys.

\section{REFERENCES.}

I. ChESTER. Report of the bacteriologist of the Del. College Agric. Exp. Station. I S99-1900. (C. reports disease in chickens).

2. Cushman. Nature of black head in turkeys. Report R. Island Agric. Exp. Station. 1894. p. 199.

3. MOORE. The direct transmission of infectious entero-hepatitis in turkeys. Circular No.5, U.S. Bureau of Animal Industry. 1896.

4. Smith. Infectious entero-hepatitis in turkeys. Bulletin No. \&, U. S. Bureau of Animal Industry. is95. 
SURRA.

Synonyms. Relapsing fever of equines ; pernicious anaemia of horses.

s 196 . Characterization. Surra is an infectious disease of solipeds and camels caused by a flagellate protozoon. It is determined by a continuous fever with alternate paroxysms and intermissions, with a general or localized eruption of the skin, petechiae of the mucosae and more or less subcutaneous oedema. There is rapid emaciation and great weakness. It is usually fatal. It attacks horses, asses, mules, goats, dogs, cattle and rats. It can be inoculated into other animals such as rabbits and guinea pigs. From an economic point of view it is reported to be essentially a disease of horses.

\$ 197 . History. This disease appears to have been known for many years to the natives of the low lands on both sides of the Indus on the northwest frontier of India. Haig appears to have observed it in Persia in is 76 . In 1880 , Evans found sereral cases of it in the Dera Ismael Khan country. He was the first to describe it and attribute its cause to an animal parasite which he discovered in the blood. In 1885 , Steel met with a disease among mules in Burma which he regarded as identical with Evan's surra, and which he believed to be relapsing fever. In is88 there was an outbreak among the Bombay Tranway Company's horses. Since then surra has become epizoötic in Bombay. Lingard reports that thousands of ponies, liorses, camels and asses died from it during the rains of 1893 and I894. Its ravages in the Punjab and Northwest Provinces during 1895 are reported to be appalling.

S I 98 . Geographical distribution. It is a disease of Asia and Africa. It is reported that "the distribution of this malady seems to be entirely influenced by the physical aspect of the country; being far more prevalent in those parts where floods and inundations occur than in the higher and dryer portions', (Pease). If the identity of surra with the tse-tse fly disease is accepted, as it seens to be, it has a wide distribution in Central Africa.

Surra does not exist in the United States, but because of its prevalence and long standing in the Philippines it is liable 
to be introduced into this country. For this reason its nature should be understood by Anerican veterinarians and pathologists.

\section{199 . Etiology. There}

is little or no doubt that surra is due to the presence in the blood of a flagellated infusorian, Trypanosoma Evansi. This organism is invariably found during the paroxysms of the disease in the blood of animals which have acquired surra either naturally or experimentally. Although blood containing these infusoria readily communicates the disease to susceptible animals it entirely loses its virulence when it is filtered through porcelain, so as to free it from the parasite. The disease can be transmitted to liealthy, susceptible animals even of differ-

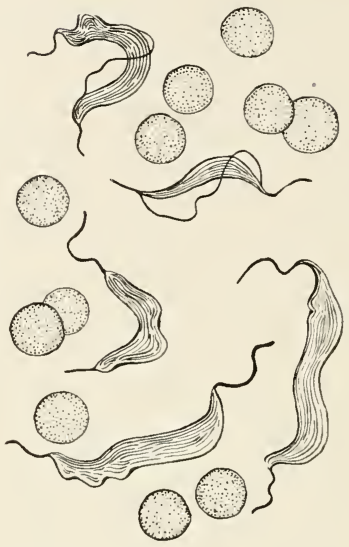

FIG. 67. Trypanosoma Evansi, highly magnified. (After Evans). ent species, with the unfiltered blood of a diseased animal. The microscope reveals the infusoria in vast numbers moving with great activity in the blood. When this acute stage has passed the organisms disappear; the temperature falls; the severity of the symptons abates ; and there is an intermission, during which, at the beginning of the attack, the patient may appear in good health. Although the blood during an intermission may appear under the microscope to be absolutely free from the parasites, its inocnlation into susceptible animals will, as a rule, produce the disease. The blood of surra affected horses loses its power of transmitting the disease by inoculation in about eighteen hours after death.

Under the microscope, these parasites are detected in a drop of blood by an irregularly intermittent and characteristic quivering of some of the red blood corpuscles, which become much 
altered in form. The leucocytes remain unchanged in appearance. After a further and careful examination of this slightly quivering blood a minute thread-like organism with eel-like movements, emerges from the mass of corpuscles. It may be seen apparently tugging with all its might at a red corpuscle endeavoring to detach it from its rouleau. The question of the manner in which these parasites interfere with the health of the affected animal has not yet been settled. When they are outside the animal body and in a dry state, they are killed or rendered inert by prolonged atmospheric heat.

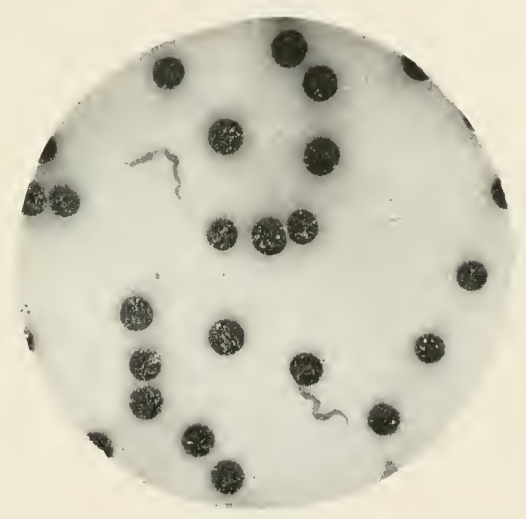

FIG. 68. Photograph of blood of horse containing Trypanosoma. (Taken by Smith and Kinyoun.)

The contagium of surra is fixed and can be conveyed only by inoculation or ingestion. Stagnant water and grass growing on recently inundated land form favorable resting places for this organism. It is probable that flies act as carriers of surra from infected animals to sound ones, especially if the latter have open wounds.

$\$ 200$. Symptoms. The symptoms as given by Lingard are as follows. "The chief symptoms, in addition to the 
ever, are the occasional appearance of an urticarial eruption, general or localized, closely following the first rise of temperature, but which may make its appearance at any time during the course of the disease; then the presence of petechiae on the mucous niembranes, chiefly that covering the membrana nictitans, lachrymation and the exudation of a semi-gelatinous nuaterial into the subcutaneous and other connective tissues. There is rapid wasting and great weakness, although in the majority of cases the appetite renains good throughout, no matter how high the fever. There is extreme pallor of the visible mucons membranes, and this is followed at a later period by yellowness. From first to last there is progressive anaenia; the blood at first presents a normal character, but after a varying period of time it undergoes marked changes. The white corpuscles are increased in number and the red corpuscles usually cease to form normal rouleaux, lose their individuality and run together forming irregular masses. They are at first dark, but gradually, as the disease advances, almost entirely lose their coloring matter and become pale."'

The respective duration of the paroxysns and intermissions is very irregular. Lingand puts it down as from one to six days. He states that in a few experimental horses the paroxysms lasted from eighteen to twenty-two days.

The period of incubation seems to be liable to great variations. It may be put from six to eight days after inoculation or ingestion of blood taken from an animal suffering from surra. It appears from Lingard's investigation that the period of latency may be prolonged to thirteen days, if the blood used for inoculation has been taken from a dead animal. When the parasites lave been given in water, by the mouth, symptoms of surra may not appear for even seventy-five days. We have no exact data for determining the time required for the disease to become manifest under natural conditions from drinking surra contaminated water. Gunn states that the average duration of the disease is about fifty-two day's.

$\$$ 201. Morbid anatomy. As a rule there is great emaciation, enlargement of the liver and spleen, petechiae on various internal organs, and a yellow or amber colored jelly- 
like exudation in the connective tissue of the throat, cliest and abdomen, about the muscles and other tissues, and especially round the base of the heart. The lungs often show signs of inflammation. The mucous menbranes and other tissues are frequently tinged yellow by the coloring matter of the bile.

Steel noticed ulceration of the stomach in about two-thirds of his cases among mules in Burma. In India this ulceration has not been observed among horses as a sequence of surra.

The clinical aspect of surra is essentially one of progressive anaemia, accompanied by paroxysms and intermissions, during both of which there is a gradual decrease in the number of the red blood corpuscles and in the amount of hemoglobin in the blood, with consequent anaemia of the visible mucous membranes.

The importance of this disease renders it desirable to reprint "A preliminary note on a parasitic disease of horses," by Capt. Allen M. Smith and Dr. J. J. Kinyoun from the Army Pathological Laboratory, Manila, Oct. I7, I90I, as it gives a good idea of the appearance of the disease. The accompanying photograph showing trypanosoma was taken by Smith and Kinyoun at that time.

"On October I5, I90I, information was given us by J. W. Jobling. Assistant Bacteriologist of the Board of Health of Manila, that an epidemic sickness of an undetermined nature was now prevailing in this city, and also that he had just taken a specimen of blood from a sick animal which on examination revealed the presence of a parasite, whether this was accidental or was the causative agent of the disease in question, he was unable to say. On investigation and enquiry it was learned from the Veterinarian in charge of the corral of the Quartermasters Department, and from the City Veterinarian, that there was now, and had been, a fatal epidemic among the horses in Manila, the Quartermasters Department having lost over 200 within the past four months.

One of the corrals was visited by us on the isth inst., where we were shown, by the Veterinarian in charge, 20 horses and mules, ill with an undetermined disease. These animals presented the several stages of the malady, some were quite recently attacked, while others had been ill for over two months.

The symptoms first noticed are: impairment of appetite, constipation, fever and thirst. These are followed within a few days by a rapid and progressive emaciation. 
The temperature for the first few days ranges from $104^{\circ}$ to $107^{\circ} \mathrm{F}$., the pulse is full and strong. This may be termed the acute stage. Then begins an asthenic state, which may terminate fatally within a variable period, or by a slow convalescence. During this stage, usually within 10 days after the onset, there appears a conmencing cedema above the belly, involving the soft parts, coincident with this, or soon after, the cedema extends to the feet and legs. The pulse becomes rapid, weak and dichrotic, the respiration increased, shallow and jerky, the gait staggering. Emaciation is rapid and extreme.

The disease has a tendency to relapse, this may occur at any time, even after convalescence appears to have been fully established. The relapses are invariably fatal.

The mortality in this epidemic has been about 75 per cent for American horses and mules, and Ioo per cent for native ponies.

The gross pathology shows serous effusions into the pleuræ, pericardium, and sonetimes the peritoneum. There is also a serous exudate into the cellular tissue of the legs and abdomen. The organs are pale, but otherwise normal in appearance.

At the time of our inspection, five acute cases were examined, the duration of the attack being from six days to two weeks. All these animals presented the several clinical appearances as above described.

Blood specimens were taken from the jugular vein of each and examined microscopically, shortly afterwards. In 4 of these a parasite was demonstrable. The other was negative, but a specinen taken the following day showed the presence of this same parasite.

On the day following, specintens were obtained from 12 others, all chronic cases, with the result of finding this same parasite in the blood of four. In three they were very few, whilst in the fourth, they were present in great numbers, as niany as 20 could be seen in one microscope field. The animal from which the specimen was taken had suffered a relapse.

It would appear that the parasite may disappear from the peripheral circulation, or exists there in such few numbers that it is not easily demonstrable, after the acute stage has passed. It would require repeated blood examinations to decide this point.

Description of the parasite. The parasite resembles a whiplike worm, having much the appearance of the Trichocephalus Dispar, its length is from to to 14 mikrons, and is from 1 . to 1.2 mikrons in diameter through its body, the neck is nearly $\frac{1}{2}$ its length, tapering gradually to a point representing the mouth (?). It has a limiting membrane, which is well defined, the contour is in most cases, symmetrical, but in some the body line is quite irregular. The larger part of the parasite (body) contains granular material and clear spaces, which latter vary in size and number; they are irregularly distributed, and may encroach on the wall so as to cause irregular ontiine. The granular material does not extend to the neck. 
The parasite is actively motile, having both a vermicular, (contractile) and spiral movement. It moves forward in a very peculiar manner, the long whiplike process is thrust forward by a spirillar notion, followed by a contracture of the body.

We have not so far been able to determine its intimate structure, further than the limiting membrane, and the protoplasmic substance of the body.

Two sizes of the parasite have been seen in all the specimens examined, the larger appears to be more numerous, and contains considerably more granular material than the smaller, and usually two or more vacuoles. Whether these two sizes represent male and female, has not been determined. We are inclined to believe from our observations, that these do represent the male and female, because we have observed in more than half the fresh specimens, the joining of a large and small parasite in such a way as to appear to be something more than accidental.

The pathological changes caused by this parasite is a rapid distruction of the red blood celis, causing an acute ancemia. The changes occur in the blood coincident to the invasion of the parasite. In one horse which had been ill seven days, the red blood cells numbered $3,500,900$, the white, I4,500. In another, ill six weeks, the red blood cells were $3,200,000$, and the white were 13,900 . The blood of a healthy horse, taken as a comparison, gave red blood cells, 6,900,000, white, 9 , Soo. There is also a slight diminution in the amount of hremaglobin, about $\mathrm{S}_{5}$ per cent.

After convalescence has been fully established, no parasite can be found, the blood gradually assumes its normal constitution.

The parasite is not confined to the blood, as it can be demonstrated in the serous effusions.

It is quite easy to detect, all that is necessary is to make a microscopical examination of fresh blood films, a $\frac{1}{6} \mathrm{in}$. objective will suffice. Dried films, fixed and stained with any of the nuclear dyes.

The organism appears to be a strict parasite. It lives but a short time after removal from the body, the longest time which it has been kept alive in blood serum, was not more than ten hours.

The parasite has many of the properties in common with the filaria, and resembles more nearly that of filaria perstans, only it is smaller, and its movements dissimilar. Yet on the other hand, the clinical history of animals infested by it, the changes occurring in the blood, the lesions observed in post mortem, point very strongly towards its classification with the spirochrete.

The mode of transmission has not yet been studied. It does not appear to be highly contagious, as it does not appear to spread from one to another, even under the most favorable circumstances.

It more nearly resembles malaria in this respect. It is more than probable that its extra corporeal state is different, or another supposition 
equally tenable, is that its intermediate host is some insect, such as the fly or mosquito."

\$202. Differential diagnosis. Surra is to be differentiated from anthrax. A history of the case or outbreak together with the chronic course and intermittent temperature in surra will usually suffice to determine the nature of the disease. If no history or symptoms are known, the diagnosis can be made post-mortem from the bacteriological examination of the tissues or blood as Bact. anthracis is readily found in cases of anthrax.

\$203. Prevention. Lingard has demonstrated quite conclusively that one attack does not prevent a horse from a subsequent one. The experience in the surra infected districts shows that the best way to prevent the occurrence of this disease among horses is ( $\mathrm{I}$ ) to see that their water supply is pure ; (2) to avoid giving them grass or hay taken from marshy or inundated ground; and (3) to exclude the excrements of rats from the grain. Lingard found that arsenic has a decided effect in diminishing the number of surra organisms in the blood of affected animals.

$\$$ 204. Trypanosomiasis. Stiles has introduced this term to describe an infection with parasites belonging to the flagellate family Trypanosomidae. The term is analogous to Taeniasis or Coccidiosis. There are a number of different kinds of trypanosomiasis now recognized and considered by certain writers to be very closely related being caused by species of parasites belonging to the same genus. Among these Stiles has mentioned the following, riz. :

I. Surra. A disease of equines, camels, elephants and certain other animals in India, attributed to Trypanosoma Evansi.

2. Nagana, nygana or Tsetse-fly disease of Africa. Affecting cattle, horses, mules, asses, antelopes, camels and certain other animals. It is attributed to Trypanosoma Brucci.

3. Dourine or maladie du coït of Algiers, France and Spain. It attacks the horse and the ass.in particular, but may be transmitted to certain other animals; it is attributed to Trypanosoma equiperdum. 
4. Mal de caderas of South Africa. It affects horses, asses, cattle, hogs and certain other animals, and is attributed to Trypanosoma equinum.

5. Rat trypanosomiasis attributed to Trypanosoma Lewisi. By some authors this parasite is alleged to be identical with the horse surra organism, but it is quite certain that rats may harbor a distinct species.

Until the results of further investigations are recorded it is deemed best to consider these as distinct infections.

\section{REFERENCES.}

I. BuRke. Surra or progressive pernicious anæmia. Vet. Jour., London Vol. XXV. IS87.

2. BuRke. Surra, pernicious anænia in the lower aninals. IVet. Jour., London. Vol. XXVI. p. 309.

3. BuRkE. Microorganisms and disease, especially with reference to the question, What is the patlology of "surra" in animals? Vet. Jour. and Ann. Comp. Path. London. Vol. XXVIII. (1889). p. 25.

4. Dirhas. Tsetse disease. Velerinarian. Vol. LXXI. (ISgs).

5. EVANS. Report on surra disease in the Dera Ismail Khan District. ISSo. Military Department.

6. Evans. On a horse disease in India known as "surra," probably due to a Hæmatozoon. Vet. Jour., London. Vol. XIII. (ISSi). July, Aug., Sept., Nov.

7. HASSALL. Bibliography of surra and allied Trypanosomatic diseases. Butl. No, 12, U.S. Bureau of Animal Industry. 1892. p. 132.

8. KANTHACK. On nagana or tsetse fly disease. Report made to the tsetse fly committee of the Royal society, etc. Proc. Royal Soc., London. Vol. LIV.

9. LINGARD. Report on horse surra. I893. (Bombay.)

10. LINGARD. Report on "surra" in equines, bovines, buffaloes and canines, etc. Rec. de med. zet. Par. 8. Vol. Vill. p. 377.

I I. NocARD. Sur les rapports qui existent entre la dourine et le surra ou le nagana. Comp. rend. Soc. de biol. Vol. LIII. (19oI).

I2. RANKING. A preliminary note on the nature and pathology of the disease known as "surra" affecting horses and mules in India. Vet. Jour., London. Vol. XXXII. (ISgi).

13. SALMON AND STILES. Emergency report on surra. Bulletin No. t2, U. S. Bureau of Annual Industry. ISg2.

I4. STEEL. On relapsing fever of equines. Vet. Jour., London. Vol. XXII. ( IS86).

15. STEFL. Report upon an obscure and fatal disease among transport mules in British Burma. I 885.

I6. SMITH AND KINYoux. A preliminary note on a parasitic disease of horses. Army Pathological Laboratory, Mlanila. Oct. I7, I9or.

17. Strles. Trypanosoma in a new role. Hm. Med. Vol. III. (I902). 
DOURINE.

Synonyms. Venerial disease of solipeds ; equine sy philis ; maladie du coit; chancerous epizoötic; breeding paralysis : epizoötic paraplegia.

\$205. Characterization. A contagious affection of solipeds, transmitted by copulation and attended by specific lesions of the generative organs and nervous system, such as local venereal swellings, chancerous ulcers and cicatrices, dementia and paralysis.

The disease is essentially an equine one, although the following species are susceptible to experimental inoculation, namely ; dogs, rabbits, rats, mice and asses. While the horse shows the greatest susceptibility, the ass is comparatively resistant to the infection.

\$ 206. History. Dourine has existed for a long time. It was first clearly described in 1796 by Ammon who found it in the royal stud at Trakchnen in Northern Prussia. We have later, I80r and 1807 descriptions of the disease in the same locality. It was found in Bromberg in 1817 to 1820 , in Austria and Bohemia in $182 I-8$, in Syria in 1821 , in Switzerland in 1830 , in France in $1830-32$, in Siberia in $1833-40$, in Italy in 1836 , in Russia in 1843 , in Poland in $1830-40$, in Algiers in $1847-55$. In Syria and Asia generally it is reported to appear perennially.

It is not known to have invaded Belgium, Scandinavia, England, South America or Australia. All indications point to Asia and Northern Africa as the home of the disease where it still appears perennially.

It was found in Bloomington, Ill., in 1882 . The first animal showing the disease was a brown stallion that had been imported from France. In this locality it spread to a considerable number of breeding mares and stallions. The disease was very largely stamped out of that region by a rigid quarantine of diseased and exposed animals. Some exposed animals had, however, left the district, and it is not surprising that isolated centers of infection are occasionally found.

$\$$ 207. Etiology. Thanhoffer found in the blood, vaginal mucus, testicle, semen, spinal fluid and roots of the dorsal 
and lumbar nerves bacteria, especially streptococci and less constantly bacilli, to which he attributed the cause. More recently Schneider and Buffard have apparently demonstrated that a protozoon, one of the Trypanosomae, is the specific pathogenic agent.

The Trypanosoma varies greatly in form at different stages of its growth. In the exudate of the ear tumefaction, without as yet other symptoms, it is found as minute granules in groups or larger spherical bodies resembling very large micrococci, each having a nucleus. There are larger bodies in which a delicate menbranous covering encloses one, two or three masses of chromatin which extend to form one or more points (club shaped or fusiform). Each chromatin mass has a nucleolus on its outer surface or slightly apart from it. Twenty-four hours later there may be added, (I) short, thick chromatin bodies with two slightly undulating, pointed, membranous prolongations ; (2) more delicate, fusiform bodies, each with one chromatin nncleus, a detached nucleolus, and the membrane prolonged into two actively moving flagella; (3) larger pyriform bodies with chromatin nuclei and nucleoli and the membrane prolonged into one or several flagella; (4) fusiform bodies thick or delicate, each having a chromatin nucleus and nucleolus, and arranged singly or in groups of two, four, six or more united together at one end and diverging at the other. These last are 20 to $30 \mu$ long by 1.5 to $2 \mu$ broad and perhaps may be the adult form of the parasite from which the small granular or spore forms found in the most recent lesions are derived. The fusiform outline, the deep staining central mass with its adjacent nucleolus, and the pointed or flagellated membranous prolongations, more or less motile or undulating are characteristic features.

In its morphology and evolutionary forms the trypanosoma of dourine has not been shown to differ from that of surra. The granule form, the spherical, the club shaped or pyriform bodies, the fusiform with more or less stellate groupings seem to be generic characteristics. The specific distinction is found in the pathogenesis as shown by the two diseases (surra and dourine). 
In the active cutaneous or mucous lesions the parasite is usually found abundantly in the blood, semen, milk, vaginal secretions and the erosions of the vaginal mucosa and penis. During intermissions, however, and in the absence of local lesions, the parasites are not found in the blood on microscopic examination, yet the inoculation of the blood into a dog will usually produce the disease. The parasite disappears from the blood and tissues very rapidly after death, so that, to prove successful, inoculations should be made from an infected individual before or immediately after death. They are not effective after forty-eight hours.

Schneider and Buffard, Nocard and others found the trypanosoma in the blood and exudates of horses, asses and dogs, suffering from dourine. They failed to find it in the same localities in animals of the same species which were free from dourine. The infected blood preserved for 24 hours in sealed glass tubes, and then inoculated into dogs produced characteristic symptoms and lesions with the many trypanosona in the blood. Inoculation into two other dogs, with the same material, but at the end of 48 hours, produced a slight transient hyperemia only, without local lesions or propagation of the parasite in the blood. The blood from the same animal inoculated after fifteen days gave negative results.

$\$ 208$. Symptoms. The first local changes in the genital organs begin after a period of incubation, according to Maresch, of from eight days to two months. The first symptom in the stallion consists in the swelling of the glans penis. Reddish spots, vesicles and ulcers may occur on the outer surface of the penis. The meatus urinarius is reddened and swollen and exhibits a mucous discharge. The animal has a continuous desire to micturate and frequently manifests sexual excitement. The swelling also spreads from the penis to the sheath and scrotum in which case the testicles become inflamed. Finally, the inguinal glands and lymph vessels become involved. These local affections may, as the disease advances, almost entirely dissappear. In some cases the external changes are absent, as the mucous membrane of the 
urethra is first affected: the only visible symptoms being strangury and a mucous discharge from the urethra.

In mares, the disease begins with a doughy or tense swelling of the pudenda, which frequently spreads to the udder and inner surface of the thighs. The mucous membrane of the vagina is red in spots and swollen, sometimes thickened by gelatinous elevations and covered with a turbid and orange colored secretion. Sometimes nodules, vesicles and ulcers are observed on the mucous membrane. They are, however, frequently absent. In mares, the local manifestations are often insignificant. The mucous membrane in the neighborhood of the clitoris is more congested than at other parts and the clitoris itself is swollen and erect. At the same time affected mares show excessive sexual excitement. They frequently suffer from strangury and after considerable straining urine is discharged in small jets.

Instead of urine small quantities of sticky, discolored mucus are discharged. The animals incessantly shake their tails and open and close the vagina in rapid succession, showing the clitoris as mares do in season. The discharge often exerts a corrosive action on the tail and legs. In severe cases the neighboring lymph glands become inflamed and swollen as well as the udder, on which abscesses may appear. The swelling may even extend to the hypogastrium.

The general symptoms develop only after weeks or even months; their appearance is often delayed until the local symptoms have disappeared. At first the animals are depressed and weak, they frequently continue to lift up their hind feet, alternately, so as to try to avoid putting weight upon them, knuckle on their fetlock joints and lose control over the movements of their hind legs while walking.

It is reported that stallions especially suffer from an uticaria in the form of sharply-defined, round, flat emiuences which may be raised the breadth of a finger above the surface and which may vary in size from two to four centimeters or more in dianteter. These eminences are caused by a serous infiltration of the papillary layer of the skin in the neighborhood of a small artery and are evidently of a vaso-neurotic 
character. They often appear and disappear very rapidly and may shift their position. Usually they persist for several weeks during which time they become moderately hard and then slowly disappear. Their favorite sites are the croup, neck, shoulders, chest and abdomen.

Later in the course of the disease, a progressive paralysis of the hind quarters combines with excessive emaciation. The animal has a staggering gait and often gives way on the pasterns and at the knees, can raise itself from the ground only with difficulty-and sometimes falls down unexpectedly. The affected stallion is unable to cover, as he can neither mount a mare nor get an erection. Some patients exhibit permanent tremblings over the whole body or local paralysis as for instance, that of the lips, ears and eyelids. Hyperaesthesia of the skin is observed particularly in stallions and with it is extensive pruritis, so that the animal continually rubs itself, bites the affected parts and thus produces extensive sores on the skin. The patient becomes extremely emaciated especially in the hind quarters so that the outlines of the pelvic bones and ribsbecome very prominent. The skin becomes dry, the hair is ruffled and loses its gloss. Some animals manifest pain when the lumbar region is pressed. The senses become more and more blunted and the eyes assume a staring and expressionless appearance. As the end approaches the patient persistently maintains a recumbent position and finally dies from the effect of secondary lesions such as hypostatic inflammation of the lungs, septicaenia or perhaps general cachexia. Sometimes in the final stage the patient suffers from nasal catarrah with swelling of the submaxillary glands and conjunctivitis. Severe internal inflammation of the eyes has been observed. The appetite continues longer than any of the other normal functions.

$\$$ 209. Morbid anatomy. In the early stages there are phlegmonous or oedentatous swellings of the sheath, scrotum, penis and inguinal glands and a yellowish liquid effusion into the scrotal cavity. The skin covering the parts may show a papular or vesicular eruption or if this has passed a mottling with white spots shows where these lesions have been. Later, 
the inguinal glands shrink and become firm owing to the development of fibroid tissue. The testicles which are either swollen or shrunken, contain foci of suppuration or caseation. The connective tissue of the epididymis and the cord is the seat of a gelatinous exudate. The walls of the scrotum may be greatly thickened and the seat of abscesses or of caseous degenerations. In advanced cases the testicles are usually abnormally small even if the scrotal mass is enormously distended. The sheath and penis may be the seat of more or less numerous wlcers and swellings. Contraction and contortions of the penis are not uncommon. It may, however, retain its normal dinensions. The walls of the lymphatics in the inguinal region may be the seat of hyperplasia, the thickening causes them to stand out like cords as in glanders. In the advanced stages the muscles, especially those of the hind limbs, become pale and atrophied. The nerve centers undergo profound changes which have been studied by Thanhoffer. The pia mater in the affected part of the spinal cord is the seat of active congestion and thickening. The central canal of the cord is dilated more at one point than another, contains more than the normal anount of liquid and the neuroglia around it is thickened and fibrous. The substances of the cord, both white and gray, show congestion, blood staining, at points foci of softening and at others induration (hyperplasia of the neuroglia). The nerve cells are nodified in various ways, some being granular, some discolored by fine granular pigment, some having enlarged and multiplied nuclei and some show vacuoles. The nerve filaments often show a granular degeneration extending from the nerve cell to the axis cylinder. The latter is liable to be raricosed or enormously enlarged. In the affected portion of the cord leucocytes are numerous and there is often hyperplasia. The neuroglia tend to increase and apart from the foci of softening tend to give a special firmness to the substance. The subarachnoid and subdural fluid is increased and there may be at the roots of the spinal nerves, especially in the dorsal and lumbar regions, a gelatinoid exudate investing the nerve, distending the connective tissue beneath the neurilemma and eren occupying the interval between the nerve filaments. 
Sometimes large corpuscular bodies are found between the nerve fibers.

The cerebral meninges are congested and opaque. Foci of softening are by no means uncommon and the cerebral ventricles contain an abnormal quantity of fluid.

The bony tissue generally has lost its consistency and the medullary matter may be unduly reddened. The large joints contain an excess of synovia of a somewhat pinkish color. The ligaments of the hip joint are often congested, thickened and softened. The articular cartilages may even show areas of blood staining.

The intestines are usually nearly empty, soft, pale and flaccid. Ruthe has in one case observed rounded ulcers on the mucosa.

The mesentery is thickened with an infiltration and it has a yellowish discoloration and the mesenteric glands are usually enlarged, softened and friable though sometimes firm and contracted. The lymph glands adjoining the generative organs are often swollen, pigmented and studded with foci of caseation, varying in size from that of a pea upward. The liver is softened, hyperemic or fatty. The spleen is small. The kidneys are usually large, pale and blackened. The thoracic organs may show little change, though hypostatic congestions and foci of caseation or suppuration may be present. The blood is light colored and forms a loose, pale clot. There is a diminution in the number of red blood cells and a relatively large increase in the number of leucocytes.

In the mare, in addition to the lesions in the internal organs and blood, the following may be noted in connection with the generative system. Phlegmons or oedematous swellings, or ulcers on the lips of the vulva and on the vulvar and vaginal mucosae. The parts become variously distorted. A crop of pustules or vesicles, which run into ulcers may appear on the urethral orifice, the vulva and adjacent skin. The mammary glands are sometimes inflamed, oedematous and tender, with suppurative or necrotic foci. The adjacent $1 \mathrm{ymph}$ glands are enlarged by infiltration or contracted by sclerosis 
In the dog the symptoms and the iesions resemble those in the horse.

$\$ 210$. Prevention. The prevention of this disease seems to rest in the isolation of all affected animals. It is important, therefore, that its diagnosis be made at the earliest possible moment.

\section{REFERENCES.}

I. Buffard and Schneider. Prophylaxie de la dourine et exposé de faits nouveaux intéressant cette maladie. Jour. de Med. Vet. et de Zootch. rgor.

2. Favil, Extirpation of maladie du coit. Annual Report, Bureau of Animal Industry. 1895-6, p. 13 and 62.

3. THANHOFFER. Über Züchilähme. Wien. IS8S.

4. Wilson-Barker. Maladie du coit in Nebraska. Vet. J. Lond. Vol. XXXV. (I892). Vol. XXXVI. (1893). 
CHAPTER VII.

\title{
INFECTIOUS DISEASES FOR WHICH THE SPECIFIC CAUSE IS NOT YET DETERMINED.
}

\author{
RINDERPEST.
}

Synonyms. Contagious typhus; steppe murrain; cattle plague.

SII. Characterization. Rinderpest is the most fatal disease affecting cattle. It is a specific eruptive fever occuring both sporadically and in epizoötics. It is characterized by a more or less typhoid condition, with lesions largely located in the mucosa of the digestive tract and skin and by the infectious nature of all of the tissues, secreta and excreta. It is a disease peculiar to cattle, although other ruminants are susceptible to it.

S212. History. Rinderpest seems to have been brought to western Europe by the importation of cattle from central Asia as early as the fourth century. It is supposed that it had long existed on the steppes of central Asia and eastern Europe. The first great epizoötic of which there seems to be records occurred about 1709 and spread over nearly all of the countries of Europe. It is reported that $1,500,000$ cattle died from its effects during the years from I7I t to I 7I4. Ramazzini seems to have been the first (I 7 I I ) to accurately describe the symptoms and lesions.

It was in connection with this disease that the first veterinary police regulations were instituted and it is stated that because of the ravages of this affection that Veterinary Colleges were first established with Government aid. In the latter half of the eighteenth century rinderpest was prevalent in nearly all of the countries of Europe. During the years 1740 
to 1750 it was estimated that three million cattle died. The importance of this disease is shown in the fact that in Italy alone during the year 1792 from three to four million of cattle died from its effects. In the years i $8+4$ to I $S_{45}$, Russia is said to have lost a million of cattle from this disease alone. Nearly all of the countries of Europe have from time to time lost heavily from it. It is stated, however, that England and Germany have practically freed themselves from it. Preventive inoculation against this disease was introduced into England by Dodson as early as $17+4$ and later, by Courtivron, into France.

\$213. Geographical distribution. Rinderpest is a well known cattle plague in Russia and the steppes of central Asia. It has extended from time to time from its home in Russia and Asia, to nearly every country in Continental Europe and Asia. More recently it has occured in southern - Africa and the Philippines. It has not been introduced into the United States or other American conmtries. At present, it is not known to exist in England, although in the past she has suffered many destructive epizoötics.

$\$ 2$ r4. Etiology. The specific etiological factor of rinderpest is not known. Several investigators have isolated and studied various species of bacteria from the tissues of animals dead from this disease. Semmer of Dorpat has always found the same organism in the lesions. His results do not appear to have been verified. There seems to be no doubt in the minds of those who have worked on this disease that it is caused by a specific microorganism, most likely a species of bacteria. Kocli makes the following statement concerning the etiology of rinderpest in the second report of his investigations in South Africa in 1897. "All efforts to find by means of the microscope, as well as through cultivation, a specific microorganism in the blood have as yet been fruitless. I also did not succeed in finding any specific microorganisms amongst the microbes which the mucus from the nose, the secretions from mucous membranes, and the contents of the intestines naturally contain in large numbers."

The virus may be transmitted from the sick to the healthy 
individuals in a variety of ways, both direct and indirect. It is said to be present in the various excreta of the diseased animal, such as the discharges from the nose, the saliva, the urine and the feces. It retains its vitality outside of the body in a moist state for months, even a year or more, according to some authorities. Hence feces and the fodder and bedding soiled by the discharges may convey the disease. When dried, however, its vitality is said to be lost in a few days. Persons may carry the virus on their shoes, clothing and farm implements. Even small animals, such as cats and rats, which frequent barns and stables hare been looked upon as carriers of the infection.

It is also claimed that animals after having passed through one attack of the disease are able to resist successfully future attacks. Inoculation with the virus is said to produce immunity, but the process of inoculation itself is followed by death in many cases. The disease is reported to havedereloped after feeding hay a year after it has lain in an infected stable. It is destroyed at a temperature of $\mathrm{I} 3 \mathrm{I}^{\circ} \mathrm{F}$. but in animal tissues it resists putrefaction.

\$2I5. Symptoms. The symptoms of rinderpest are those of a severe, acute, infective disease. The period of incubation is stated to be from six to nine days. At first the temperature rises to 105 to $106^{\circ} \mathrm{F}$. and remains near that point with but slight variations until other symptoms develop. The pulse is small, beating from 60 to 20 per minute. There is great debility, decrease in the yield of $111 \mathrm{ilk}$ and loss of appetite : rumination becomes disturbed and the animal may have slight attacks of shivering.

After these preliminary symptoms there are well marked rigors, respiration becomes accelerated and the visible mucous membranes assume a scarlet color. There is entire loss of appetite, arrest of the function of rumination, thirst, constipation with the feces dry and covered with mucus. Sometimes there is slight colic. Later there is a discharge which is first serous and subsequently sero-mucous from the eyes, nose and vagina. The saliva flows from the mouth. The feces gradually become thinner until violent diarrhoea accom- 
panied by colic sets in. The evacuations become foetid, viscid and sometimes mixed with blood. The animal becomes rapidly emaciated, staggers when walking, is very sensitive to pressure on the loins and lies down a great deal. In exceptional cases the patient manifests nervous symptoms. Others exhibit violent dyspnea and symptoms of severe inflammation of the lungs.

As the disease advances characteristic changes are to be seen in the mucous membranes. Red patches which may be flat or in the form of wheals and which quickly become covered with a greyish white, loose crust appear on the mucous membrane of the lips, tongue, cheeks, gums, nostrils and vagina. The uppermost epithelial layer consequently becomes opaque and yellowish gray spots develop on it. Less frequently crusts are formed from the tumors by their caseous disintegration. The crusts on being shed, leave dark red hollow places, the so called erosion ulcers, which readily bleed. In slight cases of the disease there may be no crusts or erosions. It is stated that sometimes an eruption in the form of very minute pimples and pustules occurs on the abdomen, inner surface of the thighs, perineum and ndder. In these cases it may be confused with that of variola. Pregnant animals frequently abort.

In sheep and goats the disease is milder, and its infectiousness is said to be less than in cattle, although the symptoms are essentially the same. Sheep are reported to suffer frequently from pneumonic affections when attacked by rinderpest.

\$216. Morbid anatomy. Authors differ somewhat on the lesions characteristic of this disease. Walley has pointed out the fact that none of the morbid changes are constant and consequently they vary with the stages of the epizoötic, the condition of the animal and the treatment. There is always emaciation, the muscular tissue is dark and capillary congestion is marked. All the tissues of the body may be the seat of effusions, exudations and blood extravasations. If symptoms referable to the nervous system have occurred, brain lesions will be found at post mortem.

The muscle of the heart is pale, relaxed and blood extravasations beneath the endocardium are not rare. The kidneys 
are usually the seat of congestion or ecchymoses either beneath the capsule or in their structure. There are also parenchymatous changes. The liver may be pale, congested, engorged with blood or ecchymotic beneath the capsule. The bile is thin. There are more or less parenchymatous changes in the liver cells. The lymphatic glands generally are usually enlarged and the mesenteric glands are often hyperemic or even hemorrhagic.

The structures most often affected are the mucous membranes of the digestive, respiratory and genito-urinary tracts and the skin.

Nodules and pustules are sometimes found on the skin, especially on the udder. The mucous membrane of the mouth and pharynx is congested in spots, swollen and exhibits rounded, yellowish grey, caseous plates or deposits. The removal of the plates discloses ulcerous and highly congested depressions in the mucous membrane, the so called erosion ulcers. These changes are best marked on the inner surface of the lips, lower surface of the tongue, buccal mucous membrane and gums of the inferior maxilla.

In the first three stomachs the mucous membrane is usually congested in spots and the epithelium is so loose that it can be easily detached. The contents of the rumen and reticulum are soft and those of the omasum are often dried, a condition that is met with in many other diseases. In some cases the contents of the omasum are fluid. Generally the abomasum is empty, and contains only a small quantity of tough, muco-purulent, yellow or sanious material. Its mucous membrane is highly congested, especially in the neighborhood of the pyloric orifice. The congestion is partly diffuse, partly in spots, in the form of points or streaks. Its color may be purple or reddish-brown with a tinge of slate-grey. The epithelium exfoliates. On the nucous membrane may be found small, brownish-yellow, caesous deposits, in the form of plates, which become detached in shreds, leaving indented, highly reddened areas which are studded with petechiae. The glands (peptic and mucous glands) of the stomach are swollen and show considerable cellular hypertrophy. Like changes 
are present in the small intestine, where there is a good deal of swelling and congestion of the mucous membrane with insulated, scab-like caseous deposits and erosions. In very severe cases these deposits form tube-like casts of the intestinal canal. At the same time there may be considerable infiltration of the solitary glands and of Peyer's patches which are swollen and either undergoing a purulent disintegration or becoming covered with caseous or purulent deposits.

In the large intestine the inflammatory changes are much less pronounced. They are greatest in the caecum.

The nasal mucous membrane is of a dark red color and covered with greyish-yellow, soft scabs. After they are renoved, the true tissue of the mucous membrane lies bare. Similar changes are found in the larynx and trachea, where the deposited masses are frequently purulent and of a creamy consistence. The lungs are sometimes hyperaemic, sometimes oedematous, hepatised or emphysematous. Pneumothorax and subcutaneous emphysema may be present.

S217. Differential diagnosis. It is very difficult to diagnose rinderpest from the first cases that occur especially if there is no history of infection. The diagnosis is based upon the symptoms, morbid anatomy, progress of the epizoötic and the history. The most characteristic diagnostic symptoms are the rise in temperature (which often occurs some days before other symptoms), formation of red spots and a yellow coating on the visible mucous membranes and later the development of erosion ulcers. A mucous discharge from the mouth, nares, eyes and vagina with symptoms of severe intestinal disturbances and excessive emaciation are of differential value.

Rinderpest is to be differentiated from " foot and mouth " disease in which the foot lesions are absent or where it is complicated with fever. Malignant catarrah if complicated with emphysema of the lungs. Anthrax, Texas fever, and other affections such as contagious pleuropneumonia and enteritis may be mistaken for it. The differentiation must be made from the specific nature of each disease.

$\$ 2$ I8. Prevention. In this connection the proclamation for the prevention of the spread of rinderpest in Cape 
Colony recently issued by the British Government authorities is of interest. It is as follows :

I. "Disinfection of hides, hoofs, horns, skins aud feathers. I. Hides, skins, hoofs, horns and feathers will only be allowed south of Orange River and will only be accepted for conveyance by rail after being thoroughly disinfected in the manner hereafter described at railway stations and the authorized crossing places in terms of Regulation 5 issued under Proclamation No. $3 S_{7}$ of 1596 , or at each of them as shall hereafter be notified.

2. No hides, skins, horns or hoofs shall be passed by the Supervising Officer unless accompanied by a declaration signed by the consignor (or his agent) that they are from aninals that have not died fron rinderpest. Any hides, skins, horns or hoofs not accompanied by such declaration, shall be immediaiely destroyed by the Supervising Officer at the consignor's expense.

3. Disinfection shall be supervised by the officer appointed by the Government for the purpose, but shall be performed by the owners or consignors or their agents.

4. Dried hides, horns or lioofs shall be immersed in a liquid disinfectant for at least 30 minutes.

5. Green hides and skins shall be immersed in a liquid disinfectant for a period of at least 15 miuutes.

6. The disinfectant used may be either a two per cent solution of carbolic acid or a five per cent solution of Jaye's Disinfectant, Odam's Disinfectant or Izal.

7. Feathers shall be hung up for three hours in a chamber charged with sulphurous acid produced by burning an excess of sulphur in a closed chamber.

8. Hides, skins, horns, hoofs and the packages containing feathers when disinfected, shall be marked for the guidance of the railway officials, and shall be accompanied by a certificate that disinfection has been efficiently performed, signed by the supervising officer without which the railway officials shall refuse to receive the articles.

9. When disinfection is completed, the articles disinfected shall be loaded direct into a previously disinfected railway truck.

10. Drying, salting or any other subsequent treatment of the hides or skins shall be done by the owners or their agents at their own risk."

"Disinfection of travelers or passengers. I. Travelers and passengers shall be disinfected at railway stations and at authorized crossing places, in terms of Regulations 7 and 8 issued under Proclamation No. $5^{87}$ of 1896 , by the police or other persons duly authorized by the Secretary of Agriculture in the manner hereafter described.

2. Natives shall strip and enter a bath containing 2 per cent solution of carbolic acid; they shall then be provided with a blanket or 
other suitable clothing until the clothes they are wearing have been disinfected.

3. The clothing of all natives, and the blankets, rugs, karrosses, wraps, etc., of all Europeans, whether from disinfected areas or not, shall be subjected to sulphurous acid fumes produced by burning an excess of sulphur in a closed chamber for at least 15 minutes, pending arrival and erection of a proper steam disinfecting apparatus.

4. Europeans coming from an infected farm or place or whe have otherwise rendered themselves liable to infection from contact with natives or their huts or kraals, shall be disinfected in such manner as, in the discretion of the disinfecting efficer, may be deemed necessary and their clothiug as provided by clause 3 .

5. The disinfectant to be used shall be

2 per cent solution of carbolic acid or

5 per cent solution of Jaye's Fluid or

5 per cent solution of Odam's Disinfectant or

5 per cent solution of Izal.

6. Boots of Europeans and natives shall be well dry brushed, and then the uppers and soles well wiped with the disinfecting solution by means of cloths.

7. Europeans on coming from an infected farm or place and all natives on presenting thenselves at a railway station, siding, halt or other place north of the Orange River, shall, before the station master, clerk, guard or other officer permits them to obtain a traveling ticket, hand to the station master or booking clerk a certificate signed by the disinfecting officer in terms of Form A, to the effect that they and their clothes have been properly disinfected as well as such articles and things in their possession as are intended to be carried by rail, not being articles or things actuaily prohibited to be carried south of the Orange River.

8. Any European coming from an infected farm or place or who has not in any way been liable to infection, shall sign a certificate to that effect in terms of Form B, and without such certificate shall be deemed as coming from an infected farm or place, or otherwise been iable to infection, and shall be subject to all provisions and regulations attached to persons coming from infected areas or liable to infection; and if tize above certificate contains any false statement the person signing the same will be liable to a penalty not exceeding Ioo pounds or, in default of payment, to imprisonment with or without hard labor, for a period not exceeding six months."

\section{Rinderpest.}

FORM A.

This is to certify that together with his clothes and the articles or things in his possession, authorized to be carried by rail, have been properly disinfected as required by the Government Regulations.

Disinfecting Officer. 


\section{Rinderpest.}

FORM B.

I of do hereby certify that I have not come from any farm or place infected with rinderpest, and I have not in any way, to the best of my knowledge and belief come in contact with infection.

(Signed.)

\section{REFERENCES.}

I. Danysz, Brodet and Theiler. The Vet. Journal. Vol. XLVI. (1898). p. 298.

2. EDINGTON. Report of Investigation. The Veterinary Journal. Vol. XLVI. (1898). p. 64 .

3. GanigeE. The cattle plague. London. I866.

4. KocH. Report. The Veterinary Journal. XLV. (1897) p. 204.

5. Ramazzini. Dissertatio de Contagiosa Epidemica, Padua. 17 I I.

6. TURNER AND KOLLE. Report of investigations. The Vet. Jour. Vol. XLV. (1S97.) p. 462.

7. WALLEY. The four bovine scourges. London. 1879.

\section{CONTAGIOUS PIEURO-PNEUMONIA IN CATTLE.}

Synonyms. Lung plague; pleuro-pneumonia zymotica. \$2r. Characterization. Contagious pleuro-pneumonia of cattle is a specific epizoötic disease which affects bovine animals and from which other species are exempt. When the disease results from exposure in the usual manner, it is characterized by an inflammation of the lungs and pleurae, which is generally extensive and which has a tendency to invade portions of these organs not primarily affected and to cause death of the diseased portion of the lung. In Germany, it is known as Lungenseuche. In France, it is spoken of as the peripncumonie contagieuse.

$\$$ 220. History. The first account of the appearance of contagious pleuro-pneumonia dates from the end of the seventeenth century. It is stated that it was observed in Hesse for the first time in 1693, and that its earliest appearance as an epizoötic was in I7 33 and I7I4, when it prevailed chiefly in Switzerland and the neighboring countries of Würtemberg, Baden and Alsace. There are detailed reports on its occurrence from the year 1743 . In 1773 , Haller published an essay 
on this disease, which was raging severely at that time and described the symptoms, anatomical characters and protective measures laid down by the sanitary police. In 1735 , the disease appeared in England and in 1765 in France. From I 790 it spread over the whole of Germany, France and Italy. At the commencement of the nineteenth century over all the countries of Western Europe.

This contagion is said to have been carried to Ireland from Holland in 1839 . The disease was brought to the United States at several different times. Probably its first introduction was in a diseased cow sold in Brooklyn, N. Y. in I $8+3$. It came to New Jersey by importing affected animals in $18+7$. Massachusetts was infected in the same way in 1859 .

South Africa was infected by a bull brought from Holland in $185+$ and Australia received the contagion with an English cow in 1858 . It is also reported as existing in various parts of the continent of Asia but the time of its first appearance and the extent of its distribution are very uncertain.

Some countries which had only been infected for a short time, such as Norway, Sweden and Denmark have succeeded in eradicating the disease without much difficulty by' slaughtering all affected and exposed animals. Other countries long infected and in which the contagion was thoroughly established, like Australia, South Africa, Italy, France, Belgium and parts of Germany have labored long, in some cases making no progress and in others being only partially successful. Holland was one of the first of the thoroughly infected countries to free itself from the contagion.

In the United States, Massachusetts eradicated pleuropneumonia during the period from i860 to i 866 . New York and New Jersey made an attempt to eradicate it in 1879 but were not successful. Late in 1883 the contagion was carried to Ohio, probably by Jersey cattle purchased in the vicinity of Baltimore, Md., to which place it had extended previous to I868. From the herd then infected it was spread by the sale of cattle during $188+$ to a limited number of herds in Illinois, to one herd in Missouri and to two herds in Kentucky. By coöperation between the United States Department of Agricul- 
ture and the authorities of the affected states, it was found possible to prevent its further spread and to completely eradicate it after a few months.

In I886, pleuro-pneumonia was discovered in some of the large distillery stables of Chicago and among cows on neighboring lots. This led to renewed efforts to secure the complete extirpation of this disease from the country. Congress, in 1887 , enlarged the appropriation available for this purpose and gave more extended authority. During the same year the disease was stamped out of Chicago and has not since appeared in any district west of the Allegheny Mountains.

The work of eradication was at the same time commenced in all of the infected States. Before the end of the year 1889 . Pennsylvania, Delaware, Maryland, the District of Columbia, and Virginia had been freed from the disease. More difficulties, however, were encountered in the States of New York and New Jersey on account of the larger territory infected and the density of the population. The long struggle was crowned with success, however, and the last animal in which the disease appeared in the State of New York was slaughtered early in 189 I and the last one affected in New Jersey met the same fate early in the spring of 1892 .

On the 26th day of September, 1892 , the following proclamation was issued, declaring the United States to be free from this disease.

PROCLAMATION-ERADICATION OF PLEURO-PNEUMONIA.

U. S. DEPARTMENT OF AGRICULTURE, OFFICE OF THE SECRETARY.

TO ALI, WHOM IT MAY CONCERN :

Notice is hereby given that the quarantines heretofore existing in the counties of Kings and Queens, State of New York, and the counties of Essex and Hudson, State of New Jersey, for the suppression of contagious pleuro-pneumonia among cattle, are this day removed.

The removal of the aforesaid quarantines completes the dissolving of all quarantines established by this Department in the several sections. of the United States for the suppression of the above-named disease. 
No case of this disease has occurred in the state of Illinois since Decenber 29, 1887, a period of more than four years and eight months.

No case has occurred in the state of Pennsylvania since September 29 , 1888 , a period of four years within a few days.

No case has occurred in the state of Maryland since September 18, I 889 , a period of three years.

No case has occurred in the state of New York since April 30, I891, a period of more than one year and four months.

No case lias occurred in the state of New Jersey since March 25, 1892. a period of six months, and no case has occurred in any other portion of the United States within the past five years.

I do therefore liereby officially declare that the United States is free from the disease known as contagious pleuro-pnenmonia.

\section{J. M. RUSK, Secretary.}

Done at the city of Washington, D. C., this 26 th day of September, A.D., I 892 .

The time required for its eradication was only about five years and the total expenditure was but a little in excess of $\$ \mathrm{I}, 500,000$.

$\$ 22$. Etiology. The specific cause of contagious pleuropneumonia has not been fully denonstrated. The infection may be introduced either by diseased cattle, or, less commonly, by bearers such as cattle dealers, attendants, utensils, fodder, dogs, etc. The sheds in cattle markets are very dangerous centers for the dissemination of the disease. All cattle are not equally susceptible. It is generally supposed that about one animal in four is immune The virus is spread principally by the respired air. Infected cattle are reported to be able to transmit it even during the period of incubation, before the symptoms are apparent. The disease is particulary infectious when it is at its height and the animal remains capable of transmitting the disease for eight or ten weeks or even longer, after the infection, especially when necrotic foci remain in the lungs. Walley estimates the duration of infectiousness in cases of encapsuled necrotic foci to be as long as fifteen months. It is said, but the evidence is not given, that the virus may be conveyed by the respired air for as great a distance as forty yards or more. In exceptional cases, the contagium is transmitted from the pregnant cow to the fetus. 
Pöls and Nolen, in I886, at Amsterdam, found round micrococci always present in the exuded matter of the lungs. These micrococci were about $0.9 \mu$ in diameter and occurred either isolated or in chains up to six in number. In the unstained preparation, the cocci were surrounded by a distinct envelope which could be stained only with great difficulty and which was absent from cultivated micrococci.

Arloing believed that he had found the exciting cause of the disease in various bacilli, especially in one in which he calls pneumo-bacillus liquefaciens bovis, which forms short, nonmotile rods. Arloing believes that his experiments prove that the pneumo-bacillus is the cause of pleuro-pneumonia.

In 1898 , Nocard and Roux succeeded in obtaining a very feeble growth of an exceedingly minute organism in bouillon containing cow or rabbit serum in proportion of one part serum to 25 parts bouillon, when cultivated in collodion sacs within the abdominal cavity of rabbits. With these cultures the disease was produced in cattle.

$\$ 222$. Symptoms. The symptoms are such as would be expected with inflammation of the lungs and pleurae, but they vary considerably according to the course which the disease runs. If the attack is an acute one, as is frequently seen in hot weather, the symptoms appear suddenly. The breathing becomes rapid and difficult, the animal grunts or moans with each expiration, the shoulders stand out from the chest, the head is extended on the neck, the back is arched, the temperature ranges from 104 to $107^{\circ} \mathrm{F}$., the milk secretion is suspended, loss of appetite, rumination is stopped, the animal may bloat and later be affected with a severe diarrhoea. Such cases are generally fatal in from seven to twenty days.

Very often the attack comes on slowly and the symptoms are more obscure. In the mildest cases there is a cough for a week or two but no appreciable loss of appetite or elevation of temperature. The lungs are but slightly affected and recovery soon follows Such animals may disseminate the virus for a long time without being suspected and for that reason are the most dangerous of all. 
A more severe type is commonly seen. The cough is frequent, more or less painful, the back somewhat arched and the milk secretion diminished. These symptoms increase, the appetite is affected, the animal loses flesh, the breathing becomes more rapid, the cough more painful, pressure of the fingers between the ribs produce eridence of tenderness, the hair loses its gloss and stands erect, the skin becomes adherent, the temperature rises to 103 or $105^{\circ} \mathrm{F}$. Animals thus affected may continue to grow worse and die in from three to eight weeks or they may after a time begin to improve and make an apparent recovery. The inflammation of the lung does not, as a rule, subside and the organ returns to its normal condition as in ordinary pneumonia, but with this disease the life of the affected portion of the lung is destroyed, the tissue dies and a fibrous wall is formed around it to shut it away from the living parts. The tissue, thus encysted, gradually softens, becomes disintegrated and breaks down into pus. The recovery, therefore, is only partial.

By those accustomed to examining the lungs of cattle, other and extremely important symptoms may be detected during the course of the disease. By auscultation an area of a certain extent niay be found where the natural breathing sound is diminished or entirely lost. This represents the diseased portion of the lung. In other cases a loud blowing sound may be heard, quite different from any sound produced when the lung is in a healthy condition. In some cases crepitation is heard near the border line of the diseased area and friction sounds produced by the roughened pleura may be detected. By percussion an area of dullness may be discovered corresponding to the portion where the respiratory murmur has disappeared. This loss of respiration detected by auscultation, and the dullness brought out by percussion are the most important evidences of an area of inflamed or consolidated lung.

Seriously affected animals remain standing if they have sufficient strength, but those which lie down are said to always lie on the affected side.

The proportion of animals that become affected after being exposed varies according to the virulence of the outbreak, the 
susceptibility of the animals and the length of time during which exposure is continued. Sometimes not over I5, 20 or 30 per cent of the animals exposed will contract the disease while at other times, 80 or 90 per cent may be infected. The proportion of cases in which the disease proves fatal also varies greatly-it may not exceed io per cent and it may reach 50 per cent. In general it may be said that about 40 per cent of the exposed animals will contract the disease and about one-half of these cases will prove fatal.

\$223. Morbid anatomy. Anatomically contagious pleuro-pneumonia is characterized by a progressive interstitial pneumonia with secondary hepatization of the lungs and exudative pleuritis. Usually only one lung, the left as a rule, is infected. The anatomical changes vary according to the duration of the disease.

The otherwise healthy lung shows, in the initial stage, small, circumscribed, inflammatory centers from the size of a hazelnut to that of a walnut. The interlobular tissue in it is hyperaenic, permeated by single hemorrhages and infiltrated with serum. The reddened lobules of the lungs are surrounded by bright margins, which are I to $2 \mathrm{~mm}$. broad and which are filled with a serous or lymphatic fluid. When the deposits are superficial, the pleura become opaque and covered with sliglit clots.

At the height of the disease there is a lobular pneumonia with pleuritis which is usually spread over the greater part of one lobe of the lung. The lung is considerably enlarged, of firm consistence, very heavy (weighing up to one hundred pounds), sinks in water and does not crackle when cut. Its section appears marbled, in consequence of the interstitial connective tissue having become thickened into broad lines which vary in color from orange to dirty white and which surromind the darker colored lobules of the lung. The larger lobules have a thickness of from . 5 to $2 \mathrm{~cm}$; and the smaller ones of from .25 to $.50 \mathrm{~cm}$. The color of the enclosed lobules of the lungs depends on the duration of the process and varies from brown-red to dirty yellow. The recently infected lobules have a blood-red, reddish-brown or dark brown color (stage 
of red hepatization). The color of the older ones varies from orange to yellow (yellow hepatization) and that of a still older date is gray (gray hepatization). The central foci, because they are the oldest, are usually in the stage of yellow or gray hepatization. Some of the inclosed lobules of the lungs are normal or only compressed, while others are merely hyperaemic. If we closely examine the bright interstitial lines, we find that they consist at first of an oedematous infiltration, which, later on, becomes plastofibrinous, gelatinous, indurated and finally tends to the formation of adventitious connective tissue. The lymph-spaces in the lines are dilated like lacunae and filled with a serons or fibrinous fluid. In robust animals, the exudate in the alveoli is firm; but is of a more serous character in animals of a weak constitution. In the former case, a section made through the lung will be found to be granular. Besides these changes, the other lympl vessels of the lungs are dilated, their walls are infiltrated with cells and their lumen is in a state of thrombosis. The blood-vessels frequently show thrombi and small hemorrhagic infarcts. The contents of the finer bronchi are often infiltrated with numerous white corpuscles. The bronchial glands and frequently the mediastinal glands are inflamed and swollen.

The pleurae are covered with soft, membranous, fibrinous masses, which are sometimes lumpy or crumbling and which can easily be detached. These deposits have a reticular surface and may attain a thickness of $2 \mathrm{~cm}$. If we remove them, we shall find the pleural vessels highly injected containing ecchymoses and the surface of the pleurae in a rough and uneven condition. In the thoracic cavity we find, in varying quantity, a generally inodorous fluid exudate, which may be clear or opaque and which contains flakes or lumpy masses. Similar fibrinous masses often lie on the outer surface of the pericardium.

After the disease has existed for some time, the affected parts of the lungs undergo induration, cicatrization, caseation, calcification, necrosis or suppuration. At first the interstitial infiltration becomes dense, solid and dry and changes into 
firm connective tissue which makes a crunching noise while it is being cut with the knife. In other places we have fatty degeneration, caseation, calcification or suppuration, in in which the enclosed lobules of the lungs, in consequence of the existing suppuration become gangrenous, form sequestra surrounded by sequestral cavities which have smooth walls. The dead portions of the lungs may remain unchanged in these cavities for a long time. Frequently they become softened to the consistency of a greasy, yeast-like paste. Sometimes, when they are comparatively small, they become absorbed, and a scar is formed. The hepatized lobules of the lungs rarely regain their normal condition after the absorption of the exudate. More frequently they atrophy or collapse, undergo atelectasis, calcification or softening, become necrotic or suppurating or form cavities. On the pleurae we flnd thick and wart-like hypertrophies of connective tissue, which frequently cause the lungs to adhere to the side of the chest.

The changes in the lungs and pleurae are the most important general changes in cases of pleuro-pneumonia. It is stated that we may sometimes meet with an interstitial fibrinous exudate in the liver with atrophy of the liver cells; serofibrinous effusions into the articulations, tendon sheaths, subcutis, dewlap and brisket; intestinal catarrh; areolation of Peyer's patches and ulcers on the gastro-intestinal mucous membrane.

The views of pathologists differ as to the nature of the earliest changes in contagious pleuro-pnenmonia and it is not within the scope of this work to present imperfectly developed or controverted theories. Without entering into a discussion of the various views, it is clear that there is an inflammatory condition of the connective tissue between the lobules, resulting in the exudation of coagulable ly mph. This inflammation is equally marked around the blood ressels and air tubes. It leads to inflammatory changes in the inner wall of the veins and these cause a deposition of thrombior plugs in the ressels, which prevent the return of the blood. The blood pumped into the lung tissue leaves the meshwork of capillaries around the air vesicles, enters the latter and produces the firm hepa- 
tized condition so characteristic of this disease. It will be easily understood how the different shades of color from dark red to grayish or yellowish red are produced if we bear in mind that the reins in different parts of the lung tissue are plugged at different times and that, the affected regions are in different stages of the disease.

One of the most conspicuous features in a microscopic section from a lung affected with acute contagious pleuropneumonia is the presence of intensely stained foci and lines. These lines, to which Prof. Welch seems to have been the first to call attention are visible to the naked eye and when riewed with a hand lens suggest by their peculiar curves the contour lines on a map. They are situated at the margin of and within the inflamed connective tissue which surrounds the large vessels and separates the lobules from one another. A closer examination of these lines indicates that they coincide with the boundaries of the lobules and of the individual lymph spaces of the interlobular tissue. Under a high power of the microscope they are resolved into dense masses of leucocytes in various stages of degeneration. These dense bands are presumably attracted to the connective tissue boundary of the lobules and to the walls of lymph spaces within the connective tissue by the unknown cause of the inflammation, presumably the cause of the disease itself. The space between the lines is filled with fibrin, in which very few leucocytes are found.

\$224. Differential diagnosis. It is extremely difficult to form an exact diagnosis of the disease during its development, because the symptoms which are present are few in number and by no means characteristic. The slight fever and cougl are the only symptoms of diagnostic importance in the prodromal stage. In the second or acute stage a positive diagnosis intra ritam can be made only when cases of pleuropneumonia have previously occurred or when several occur simultaneously. As a rule, a correct diagnosis can only be made by a post mortem examination. The following diseases are to be differentiated from it :-

I. Non-infectious inflammation of the lungs. This may be distinguished clinically, as a rule, by its more acute and more 
typical course and by its sporadic and sudden appearance and, anatomically, by the fact that the parts which have undergone hepatization are practically all of the same age and by the interstitial lung tissue and pleurae being but slightly involved. Nocard states that in exceptional cases pleuropneumonia may run a very acute course and show hepatization of equal ages.

2. Tuberculosis. This disease cannot be positively distinguislied clinically from pleuro-pneumonia. Tuberculin may be used as a diagnostic agent, although it is not at all certain that animals which have suffered for some time from pleuropneumonia do not react to tuberculin. After all, an anatomical examination is the only real test. Tuberculosis and contagious pleuro-pnemmonia have occasionally appeared simultaneously in the same animal. See description of lesions in tuberculosis.

3. Traumatic pneumonia or pneumonia due to foreign bodies may exhibit the same symptoms as pleuro-pneumonia. Evidence, during life, of changes in the heart caused by traumatic carditis will be conclusive in making the differentiation.

4. Broncho or interstitial pneumonia. In making a diagnosis between contagious pleuro-pneumonia, based on the lung lesions alone, it should always be borne in mind that the entire lungs should be subjected to examination. Attention should be given to signs indicating injury to the chest wall, to the possible presence of foreign bodies and to severe inflammation of the air passages which may result from the aspiration of irritant or corrosive fluids. Where these agents may be excluded special attention should be paid to the condition of the pleura and to the distribution of the disease, careful notes being made of the lobes involved. Next in order come the peculiar perivascular and interlobular thickening and the thrombosis of the veins. Care should be taken not to confound mere clots with adhesive thrombi. In acute pleuropneumonia, after death, the arteries are usually distended with clots. The different features of the hepatized and oedematous portions of the lung tissue should be carefully noted. Lastly, the peculiar exudation and infiltration in the 
connective and fatty tissue of the dorsal mediastinum and of the embedded glands should not be overlooked. With the microscope the peculiar dense cell masses of the diseased connective tissue should be looked for and the nature of necrotic tissue determined in case microscopic appearances are 110 longer reliable owing to hardening processes.

Finally it should be borne in mind that the lesions of broncho-pneumonia and the interlobular changes which may follow it may coexist with contagious pleuro-pneumonia, and that both kinds of lesions may be encountered in the same lungs. Hence, great caution nust be excercised in expressing an opinion when only a small portion of the lungs are presented for examination, because only that portion which is affected with broncho-pneumonia may have been submitted.

$\$ 225$. Preventive inoculation and eradication. In Europe inoculation has been practiced for a long time as the principal means for combatting pleuro-pneumonia. As early as the beginning of the last century it was proposed in Germany by Hausmann and others. Its employment was greatly increased by the investigations which were made in 1850 by Willems in Holland, which were published in $185^{2}$. Since that time these inoculations have been practiced in nearly every country. The literature on the subject is very copious. However, the value of inoculation as a protection against pleuro-pneumonia has not yet received a final answer.

The adrocates of inoculation, among whom we may mention Haubner, Bouley, Schïtz and others start from the well known fact that one attack of pleuro-pneumonia successfully passed through, confers immunity for the remainder of the animal's life. By inoculation, a local, specific, inflammatory process which is analogous to that in the lungs, is produced and is followed by subsequent immunity of the whole body. Haubner calculates that the mortality from the inoculation is from $I$ to 2 per cent and that the tips of the tails are lost in from 5 to ro per cent of the cases. In Holland, among 59, I8o cattle inoculated in 1878 and 1879 , the mortality amounted to only 0.66 per cent.

The opponents of inoculation, among whom we may men- 
tion Rolof, Zïndel, Kitt, M'Fadyean and others, assert that up to the present no positive case of immunity has been proved to have been obtained from inoculation. They also point to the fact that even the adrocates for inoculation are unable to give the exact duration of the immunity and consequently make several inoculations. The specific nature of the tumor produced by inoculation is disputed, because an exactly similar tumor appears after the inoculation of pus or milk. We must also bear in mind that in those countries in which inoculation has been most practiced, the disease shows no decrease, as for instance in England, where the official report lays particular stress on the doubtful value of obligatory inoculation for pleuropneumonia as in France and Belgium where inoculation was obligatory. The best procedure seems to be stamping out the disease with thorough disinfection or destruction by fire of all infected sheds and barns. The success of this method is illustrated by the eradication of the disease from the United States.

\section{REFERENCES.}

I. NocARD AND Roux. Le microbe de la peripneumonie. Recueil de Méd. V'étérinaire. March 24, I898. p. 213. (Trans, in The Veterinary Joumal, London. Vol. XLVII. (I89S). p. I47.

2. Salmon. Annual Reports of the Bureau of Animal Industry. I $\mathrm{S}_{4} \mathrm{~L}^{-1} \mathrm{Sg}$.

3. Simtr. Annual Report of the Bureau of Animal Industry. IS95-6. p. I 43 .

4. WALLEY. The four bovine scourges. (IS79).

\section{FOOT AND MOUTH DISEASE.}

Synonyms. Eczema epizoötica ; epizoötic aphtha ; vesicula aphtha ; vesicular epizoötic ; apthous fever ; murrain.

$\$ 226$. Characterization. Foot and mouth disease is a highly infectious disease of animals. It is determined by the eruption of vesicles in the mouth, around the coronet of the foot and between the toes. It is said to be more common among cattle but swine are quite susceptible; sheep, goats, horses, dogs, cats and fowls are sometimes attacked. The human species is also susceptible and may be infected by 
drinking the unboiled milk of animals suffering from the disease. The mortality is not high.

$\$ 227$. History. Foot and mouth disease was quite accurately described in the eighteenth century. Very destructive outbreaks are reported in 1809 and in the early part of the nineteenth century it was the source of much loss in southern Europe. In $188_{3}$, Great Britain lost heavily from its ravages. In I892, Prussia is reported to have had $8,000,000$ cattle affected. It was during the years 1890 and 1893 that the last severe epizoötic appeared in Germany.

$\$ 228$. Geographical distribution. This disease seems to be very largely restricted to Europe although it is alleged to have been introduced into almost every cattle raising country. Its invasion into the United States has been prevented by the enforcement of rigid quarantine measures.

229. Etiology. The specific cause of foot and mouth disease has not been clearly demonstrated. It is generally believed, however, that it is caused by a specific infection and that every outbreak starts from some previous case or cases. The virus is contained in the eruptions and given off from the mouth and feet causing its wide distribution. Animals may be infected directly by coming in contact with the disease, or indirectly by being exposed to the virus in stables, cars or other places recently occupied by infected animals. It is stated that the virus is destroyed in a short time by drying, but some writers maintain that it will persist for several months. Animals that have passed through the disease may carry the virus for several months. The infected matter may be carried on the clothing or hands of human beings and thus be transmitted to animals or men. Nilk is often the carrier of the virus.

$\$ 230$. Symptoms. The symptoms of foot and mouth disease vary greatly in different epizoötics, sometimes they are quite mild and at others very severe. The first evidence of the disease is a rise of temperature which in cattle rarely exceeds $104^{\circ} \mathrm{F}$. This follows after a period of incubation of from 3 to 5 days although in rare cases it may be shorter or longer than these limits. The mucous membrane of the mouth becomes reddened, the appetite is diminished, and rumination ceases. 
The mouth is usually kept closed and the quantity of saliva is increased. A smacking sound is not infrequently made by the animal. These symptoms are chiefly due to the pain accompanying the disease in the mouth. After two or three days the eruption appears. This consists of small yellowish-white vesicles or blisters varying in size from a hemp seed to a pea on the gums and inner surface of the lips, the inside of the cheeks, the border and under the surface of the tongue. They may become half an inch or more in diameter. In some cases the back of the tongue near the tip may be the seat of large blisters.

These vesicles burst soon after their appearance, sometimes on the first day. More rarely they may persist for two or three days. After they have ruptured the greyish-white membrane forming the blister may remain for a day or more or disappear speedily and leave deeply reddened areas or erosions which are very painful. These exposed areas may soon become covered again with the normal epithelium or they may be converted into ulcers. In this stage the saliva forms in large quantities and hangs in strings from the mouth. In eight to fourteen days the disease may have entirely disappeared.

In addition to the changes in the mouth, one or more feet may become diseased. The skin around the coronet and in the cleft between the toes becomes hot and tender and may swell. Blisters appear as in the mouth, but they are speedily ruptured and the inflamed, exposed areas are covered with a viscid exudate.

The udder, more particularly the teats, may be the seat of lesions. Some authorities regard the udder disease merely at she result of infection during milking. The vesicles are broken as they appear by the hands of the milker and the teats become covered with reddened areas, deprived of the superficial layer of the skin and are very tender. The healing, however, goes on quite rapidly. The milk is said to be somewhat changed in appearance and unfit for making butter or cheese. These are the main symptoms accompanying the uncomplicated cases of foot and mouth disease. In all such cases 
recovery is usually rapid and complete. In certain other cases, however, complications arise which are not only injurious but may be fatal. Thus the mouth lesions may be accompanied by nasal catarrh or pneumonia. The feet may become very much swollen and the inflammation and suppuration extend to the tendons and bones resulting in the loss of the hoof. Such cases are usually fatal. As a result of the general affection young calves may succumb to a secondary inflammation of the stomach and bowels and older animals may abort or suffer from inflammation of the udder.

The duration of the disease in uncomplicated cases varies from io to 20 days. Where complications occur either with the regular course or as sequelae the duration becomes indefinite. The mortality varies with the severity of the attacks, the age and condition of the animals and the treatment. Ordinarily the mortality is not high excepting in very young animals.

$\$ 231$. Morbid anatomy. The tissue changes found at post mortem vary to a marked degree. There is in certain cases hyperaemia and oedma, catarrh of the nares and mucosa of the lungs and dilatation of the heart. There may be fatty degeneration and hemorrhagic infarcts in the heart muscle. In other cases there is severe gastro-enteritis with intestinal hemorrhage. In the stomachs of cattle oval reddish areas which ultimately form ulcers appear. These often become confluent. The involved portion of the mucous membrane becomes thickened. The areas of necrotic tissue in which the ulcers appear become surrounded by a reddish line of demarcation which, in the healing process, forms a cicatrix. The lesions on the mucous membrane rarely extend deeper than the mucosa. Ulcers of a nature similar to those found in the stomach occur in the intestines. On the feet inflammations may follow the simple vesicles about the coronet. These may extend deep into the tissues, pass under the hoof and cause it to slough off, extend into the bone producing necrosis or permanent arthritis. It is stated by some authorities that in the mild non-fatal cases the obrious lesions are so slight that frequently they escape notice. In sheep and swine the lesions are more frequently restricted to the feet. 
\$232. Differential diagnosis. Foot and moutl disease must be differentiated from (I) various forms of stomatitis caused by different fungi and often referred to as sporadic aphthae, (2) from stomatitis due to drugs and injuries, (3) from actinomycosis of the tongue and (4) from variola. It must also be differentiated from simple cellulitis, often of streptococcic origin, in the subcutaneous tissue about the coronet and from the sloughing of the hoof resulting from the extension of the inflammatory process and from eczemas due to dietary causes. The means of differentiating these must be found largely in the history of the cases and in the study of the nature of the lesions themselves.

\$233. Prevention. Preventive inoculations do not seem to have given satisfactory results. The isolating of the diseased animal and the placing of the well ones in non-infected fields and stables tend very largely to the checking of the spread of the disease. The milk of the diseased animal should be sterilized before it is used.

\section{REFERENCES.}

WALLEY. The Four Bovine Scourges. I879. p. 6I.

\section{RABIES.}

Synonyms. Hydrophobia; canine madness.

$\$$ 234. Characterization. Rabies is an acute infectious disease transmitted from animal to animal or from animal to man by the bite of the rabid individual or by direct inoculation. It is not known to be contracted or transmitted in any other manner. It is characterized by a long and variable period of incubation, followed by symptoms referable to the nervous system lasting from one to ten days ending in paralysis and death, withont recognizable gross anatomical changes.

The dog is the animal most commonly affected although all of the canine and feline races seem to suffer from rabies more than other species. All warm blooded animals appear to be susceptible. It is a serious disease in man, cattle, sheep, horses and swine. An explanation for its greater frequency 
among dogs is found in their tendency to bite. A very large percentage, in fact nearly all, of the cases in man and in the domesticated animals are caused by inoculation from the bites of rabid dogs.

$\$ 235$. History. Rabies was described by Aristotle in the fourth century B. C. He wrote, "Dogs suffer from madness that puts them in a state of fury, and all the animals that they bite, when in this condition, become also attacked by rabies." Cornelius Celsius who lived in the first part of the Christian era seems to have been the first to refer to human rabies and to employ the term "hydrophobia."

The transmission of the disease by wolves to man was recorded in I59I. In I 803, and for a number of years following, it was epizoötic among foxes in Southern Germany and Switzerland.

During the latter part of the eighteenth and the beginning of the nineteenth century the disease extended over Europe and about this time it appeared in America. The first outbreak in this country was reported from Boston in 1768 . In 1770 to $\mathrm{I} 77 \mathrm{I}$ it was observed in dogs and foxes in the vicinity of Boston; in the year 1779 it appeared in Philadelphia and in the state of Maryland; in 1785 it was prevalent throughout the northern States and soon after it spread to the southern States. During the last century it has caused heavy losses among farm animals thronghout Europe and America.

It has called forth careful study from many of the ablest men in the medical professions. Among them may be mentioned John Hunter in England, Viborg in Copenhagen, Waldinger in Vienna, Hertwig in Germany and Pasteur in France. Without detracting in the least from the great work of other investigators, we may say that to Pasteur and his co-laborers, Nocard and Roux, we owe much of the knowledge which we possess of the disease at the present day.

$\$ 236$. Geographical distribution. Rabies is known to exist in almost every country on the globe. Australia is the only land which is said to be absolutely free from it. This exemption is the gratifying result of a rigid quarantine enforced against dogs imported to that island. Rabies is very 
common in France, Belgiun and Russia. In the latter country it is perhaps nore often seen in wolves than in any other part of the world. In Holland, Denmark and Sweden it is very rare. In England, it has from time to time been widespread, but at present it is practically eradicated. Salmon has found that rabies exists in nearly every part of the United States.

Inquiries which the writer has made during the last few years, by verifying current newspaper reports of rabies, shows that the larger number of cases come from certain districts in which the disease seems to have become established. Newly infected districts are constantly springing up, due presumably to the introduction in some manner of an infected dog.

The prevalence of the disease cannot be accurately determined as there are no reliable statistics concerning it. Ravenel has reported that the State Live Stock Sanitary Board of Pennsylvania has investigated $8_{2}$ cases of rabies since 1897 . Of these there were $5^{8}$ dogs, 4 horses, I 7 cows, I cat and 2 human beings. Since November I goo the New York State Veterinary College has investigated 27 cases of rabies of which I 4 were in dogs, 9 in cattle, I in horses, I in sheep and two in men. Salmon states that in the District of Columbia from I 993 to August I 900 rabies has been positively diagnosed in animals in 91 cases. These consist of 80 dogs, 5 cows, 2 horses, 2 foxes and 2 cats. In addition to these the records of the health departmient of the District of Columbia show that since 1874 there has been in the human species seven deaths. from rabies. These illustrations from the experiences of a few laboratories could be duplicated from the records of many others.

In Europe the disease seems to be more prevalent. In I 898 the official reports show 1,202 cases of rabies in animals (mostly dogs) in Germany. In I 899, 2,374 rabid animals were reported from France and 444 cases from Belgium.

$\$ 237$. Etiology. Although rabies has become recognized as a specific disease, its primary etiological factor has not been isolated or found. It is known that it exist in the brain, spinal cord and saliva of the affected animal. In cer- 
tain instances its presence has heen reported in salivary glands, the pancreas and milk. It has not been found in the blood. A very large amount of work has been done by way of investigation to determine, if possible, the nature of the specific cause but thus far positive results have not been recorded. It slould be stated, however, that a number of workers have found in the brain or cord of affected animals various forms of bacteria and Blastomycetes but in every instance the conclusions reached by the authors have not been confirmed by others. While it is true that the cause has not been recognized, the researches in recent times have brought to our knowledge many facts concerwing it. It can be removed from the saliva by filtration which indicates that it is a solid particle.

s38. Method of invasion. When introduced into an animal either experimentally or by the bite of a rabid dog, the virus renains for a time without producing either local or general symptoms. The period of incubation varies within quite wide limits. The virus penetrates the nervous system by following the nerve trunks from the site of infection to the spinal cord, then through the spinal cord to the brain. This has been proven by inoculating an aninal in one of its legs with virulent material. After a suitable time, but before the symptoms of rabies appear, the virus will be found, on killing the animal, in the nerves of the limb, and even in the part of the spinal cord into which the nerve enters, while the upper part of the cord and the brain are still uninfected. This explains the fact why the earliest symptoms, both in man and animals, such as itching, tingling, numbuess and other nervous sensations, often appear in the part of the body which received the virus. In the case of a bite about the face and head the route along the nerve to the central nervous system is much shorter. While the nerves seem to form the main route by which the virus travels, the circulation nay at times assist, especially in small animals. Inoculation into the large nerve of the leg is almost as certain to produce the disease, as inoculation directly into the sub-dural space, while injection beneath the skin of the leg is not so sure.

Resistance of the virus. The action of the virus is destroyed 
by drying and by the action of light. In dry air, protected from light and putrefaction, the virulence of the spinal cord of rabbits is destroyed in fourteen to fifteen days. When spread in thin layers it is entirely destroyed by drying in from four to five days. Sunlight destroys it in about forty hours. The loss of virulence by drying is gradual but quite regular, which fact was taken advantage of by Pasteur in the preparation of his vaccine. The virus may be preserved in neutral glycerin at ordinary temperature for a long time. Roux found that after four weeks in glycerin at $30^{\circ} \mathrm{C}$., the virus in a rabid brain had the same power as when perfectly fresh. The writer has found that rabbits inoculated with rabid brains that had been kept in glycerin from three to four weeks did not develop the disease as quickly as when they were inoculated with the freshly removed brain.

It is quite resistant to putrefaction. Galtier has found the virus active in the central nervous system of rabbits buried for twenty-three days, of sheep buried thirty-one days and of dogs buried forty-four days. Other observers have found it still active in animals buried for twenty-four days.

It is destroyed completely by a temperature of $50^{\circ} \mathrm{C}$. in one hour or $60^{\circ} \mathrm{C}$. in one-half hour. It is uninjured by exposure to extreme cold, resisting the prolonged application of a temperature from 10 to $20^{\circ} \mathrm{C}$. below zero.

Its activity is destroyed in one hour by a five per cent solution of carbolic acid, or by a i to $\mathrm{I}, 000$ solution of corrosive sublimate. Water saturated with iodine destroyes it in ten minutes.

239. Period of incubation. The period of incubation is quite variable depending on the site of the wound which is almost always a bite, the amount of rirus introduced and its virulence. In general it may be said for all animals that the period of incubation seldom exceeds sixty days, though in man and in some of the larger animals, it sonetimes, though very rarely reaches one year. A few cases of a longer period have been reported. The arerage period as given by Ravenel is as follows:

In man, 40 days. 
In dogs, 21 to 40 days.

In horses, 28 to 56 days.

In cows, 28 to 56 days.

In cats, 14 to 28 days.

In pigs, 14 to 21 days.

In goats and sheep, 21 to 28 days.

In birds, 14 to 40 days.

In rabbits inoculated subdurally with the brain from rabid animals, the writer has found the period of incubation to vary from 12 to 62 days and the duration of the disease to range from a few hours to three days. Wesbrook reports a period of incubation in rabbits to extend in one case over a hundred days. In the disease as it is naturally contracted from the bites of rabid animals, the period of incubation varies with reference to the location and extent of the bites. If the individual is bitten about the head the period of incubation is much shorter than if the injuries are on the extremities.

In the $d o g$, the period of incubation in 144 cases was clearly determined by Peuch. His table with the addition of percentages is appended.

PERIOD OF INCUBATION OF RABIES IN THE DOG.

\begin{tabular}{|c|c|c|}
\hline $\begin{array}{l}\text { Number of days of } \\
\text { incubation. }\end{array}$ & $\begin{array}{l}\text { Number of } \\
\text { cases. }\end{array}$ & Per cent. \\
\hline 5 to 10 & 3 & 2.08 \\
\hline Io to 15 & $\bar{s}$ & 555 \\
\hline I 5 to 20 & I3 & 9.03 \\
\hline 20 to 25 & 25 & 17.36 \\
\hline 25 to 30 & 13 & 903 \\
\hline 30 to 35 & 25 & $17 \cdot 36$ \\
\hline 35 to 40 & 6 & 4. 17 \\
\hline 40 to 45 & II & 7.64 \\
\hline 45 to 50 & 9 & 6.25 \\
\hline 50 to 55 & 4 & $2.7 \mathrm{~S}$ \\
\hline 55 to 60 & 2 & 1. 39 \\
\hline 60 to 65 & 7 & 4.86 \\
\hline 65 to 70 & I & .69 \\
\hline 70 to 75 & 5 & 3.47 \\
\hline So to 90 & 7 & 4.86 \\
\hline 100 to $\mathrm{I} 20$ & 4 & 2.78 \\
\hline $365 \ldots$ & I & .69 \\
\hline Total_ & 144 & \\
\hline
\end{tabular}


The somewhat popular opinion that most of the cases of rabies occur in the summer, especially in "dog days", is not founded upon facts. Rabid dogs are nearly if not quite as numerous in winter as in summer. Salmon has collected I 4, 066 cases of rabies in dogs with the months the disease occurred. The results are exceedingly interesting as the appended table shows.

CASES OF RABIES IN DOGS, BY MONTHS.

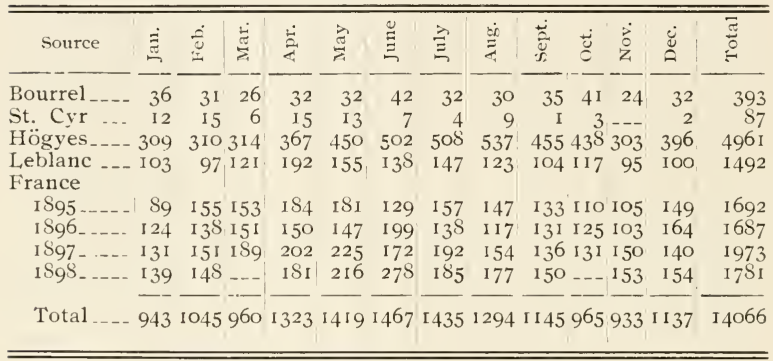

S240. Symptoms. Rabies is generally divided into two forms, furious and dumb rabies. In the first the animal is irritable, aggressive and bites nearly every object which comes in its way ; in the second the muscles of its jaw are paralyzed almost from the beginning and being unable to bite, the animal remains more quiet and tranquil. Essentially the two forms of the disease are the same, but probably owing to the parts of the brain attacked or the acuteness of the attack or both, paralysis appears much sooner in the dumb form than in the other. The saliva from a case of dumb rabies is just as dangerous and virulent as that from a case of furious rabies. Dogs affected with dumb rabies are less dangerous simply because they are unable to bite and thus to infect others.

Dumb rabies and furious rabies do not always represent two distinct types of disease. The typical cases belong to the two extremes of symptoms and there are always gradations between them. In fact, almost every case of furious rabies 
sooner or later changes to the dumb form, that is, the final stage of the disease is almost invariably paralytic. In the typical development of the dumb form, the paralysis occurs on the first day of the disease. It may not appear, however, until the second or third or even a later day.

Again, a dog does not necessarily bite everything about it even if it has rabies and its jaws are not paralyzed. It may be combative and furious all of the time or only part of the time, or not at all. There is perhaps no disease in which the symptoms may vary more than in rabies of the dog.

Furious rabies. The symptoms appear very gradually. The animal's habits and behavior are changed. It may be more restless or affectionate than usual, seeking to be near its master or mistress, fawning, licking the hand or face and apparently seeking sympathy or assistance. Such caresses are, however, extremely dangerous, for the animal's tongue, moist with virulent saliva, coming in contact with a part where the skin is thin, abraded or wounded may fatally infect the person to whom it is endeavoring to demonstrate its affection. The reported cases in which rabies has developed from such inoculations are quite numerous.

In most cases dogs first become dull, gloomy, morose, seeking solitude and isolation in out-of-the-way places or retiring under pieces of furniture. But in their retirement they cannot rest, they are uneasy and agitated, they lie down and assume the attitude of repose, but in a few minutes they are up walking about "seeking rest, but finding none." Occasionally this restlessness may disappear for a time and the animal becomes lively and affectionate; oftener it sinks into a sullen gloominess from which even its master's voice rouses it but temporarily. At this period dogs may have aberrations of the senses which cause hallucinations and lead them to think they are being annoyed by something or that some animal or person is endeavoring to injure them. They crouch ready to spring upon the enemy; they rush forward and snap at the air ; they throw themselves, howling and furious, against the wall as though they heard sounds beyond it. 
While at first the affected dog may not be disposed to bite, it becomes more dangerous as its hallucinations and delirium increase.

The disturbance of the sensations leads to chills and itching. If the place where the bite occurred is accessible, the dog licks the scar and later bites and tears the tissues. In this case it bites into its own flesh with apparent pleasure and 'satisfaction. Food is taken until the disease is considerably advanced, if it is something which can be swallowed without mastication, otherwise it is soon dropped. Difficulty in swallowing is an early symptom. Mad dogs have no fear or dread of water, they continue to drink until paralysis prevents them from swallowing.

When the furious symptoms appear, the dog leaves his home and starts upon a long chase with no apparent object in view other than to be traveling. He trots at a rapid pace, eye haggard and tail depressed. He is indifferent to the sur-. roundings. He flies at and bites persons whom he meets, but usually does not apparently search for them or even notice them if they remain quiet. Dogs in this condition may travel many miles and finally drop from exhaustion and die. Often after the absence of a day or two they return to their home, exhausted, enaciated, covered with dust, thus presenting a most forlorn and miserable appearance. Those who have pity for such an animal and try to make it clean and comfortable are in great danger of being bitten as the disease has advanced to a point where the delirium or insanity is most marked and where a treacherous bite is most common.

If the animal, instead of being allowed to escape, is kept confined, the paroxysms of fury are seen to occur intermittently or, in the absence of provocation, they may be entirely wanting. If excited it howls, rushes upon objects that are thrust toward it or throws itself against the bars of its cage and bites with great fury.

As death approaches the animal becomes exhausted and is scarcely able to stand. The eyes are dull and sunken and the expression is that of pain and despair. Paralysis appears in the jaws or in the posterior extremities and extends rapidly to 
other parts of the body. The animal, being unable to stand, lies extended upon its side, the respiration becoming more and more difficult. There are spasmodic contractions of certain groups of muscles, complete prostration and finally death.

The ordinary course of the disease is four or five days. It may be as short as two or as long as ten days.

Dumb rabies. When this form of the disease is typical, it comes on with restlessness, depression, a tendency to lick objects and paralysis of the muscles which close the jaws. As a consequence of the paralysis, the lower jaw drops, the animal is unable to close the mouth, the tongue hangs out and an abundance of saliva escapes. The mucous membrane of the mouth becomes dry, discolored and corered with dust. The aninal remains quiet, it does not respond to calls and appears to understand its helplessness. Bouley states that the animal cannot bite and does not desire to bite. When dumb rabies follows a period in which the animal has been affected with the furious form, the desire and tendency to bite may be retained even after the jaw is paralyzed.

The course of the disease is short, death usually occurring in from two to four days.

$\$$ 24I. Morbid anatomy. One of the striking characteristics of rabies is the absence of constant, recognizable lesions. The mucosa of the pharynx and larynx are congested. The spleen is sometimes enlarged and dark colored. In dogs the stomach often contains a variety of foreign matter sucl as earth, stones, pieces of iron, bits of leather, wood, etc. Axe reports finding such foreign substances present in 90 per cent of 200 cases he examined. Galtier reports such findings in from 50 to 70 per cent. In experimental animals and cattle the writer has rarely found them. It seems to be true that the obvious lesions are not constant and it is very likely that the pronounced changes occasionally found in a single organ are accidental or secondary rather than primarily related to the disease. The lesions in the brain and spinal cord are likewise variable. In some cases there is a marked hyperemia, while in others the brain appears to be normal. 
Certain investigators however have found histological changes, which to their minds have been pathognomonic of the disease. The close simulation of the nervous lesions to those due to other diseases, and the possibility of greater or less postmortem changes will foster an element of doubt in the minds of the majority of working histologists. This doubt instead of diminishing shows a tendency to grow when a review is taken of the conflicting results and opinions lield by those who have already investigated this field. It also appears that some portions of the nervous system may exhibit lesions of a pronounced character, other portions very slightly, and still others none at all, thus presenting additional difficulties.

One of the most common lesions that has been observed is of an inflammatory character, the congested blood vessels frequently showing diapedesis and, according to some, a perivascular exudation of a granular or hyaline substance. Hyperemia and lymph-stasis, although of not so much significance when taken by themselves, have been taken into consideration along with other changes. The blood ressels quite as much or even more than the nerve structures have been noted as the locus of some of the most marked changes, among which are the proliferation of the epithelial cells and of the connective tissue elements of the outer coat, with the infiltration by lymphoid cells. Such lesions may be nodular primarily, but later become diffuse. The inflammatory processes may progress to such an extent as to obliterate certain vesseis.

Pathological miliary centers have been noted not only in the axial portions of the nervous system, but in the gray matter as well. These centers are formed by lymph cells which accumulate notably around the blood vessels (perivascular) and the nerve cells (pericellular) as well. The lesions, when present, are observed most frequently in the motor centers of the oblongata and spinal cord.

The following observations were made by Babes, in 1887 :

I. "In animals dead from street rabies there are found usually a hyperæmia and an acute generalized œdema of the cerebral meninges, acute hemorrhages localized around certain 
vessels, as well as inflammatory lesions. On microscopic examination we find an increase of the plasma cells, augmentation of the reticular substance, fibrinous in character, between the several layers of the meninges.

2. The epithelium of the cerebro-spinal central canal has proliferated. In the gray matter which surrounds the canal, and especially in that of the floor, hemorrhages, sometinies symmetrical, are often found. Microscopically, we often find an obliteration or thrombosis of a ressel by a reticulated, hyaline, pigmented material or by leucocytes or hyaline globules, and sometimes a hyaline degeneration or even inflammation of the vascular tunic. The extravasated blood also contains much of the hyaline material. The hemorrhages are often limited by the lymphatic sheath of the vessels. At the same time the epithelium of the ventricles and central canal may be partially lost. This last is occasionally filled with blood or plugs, either granular or hyaline in character.

3. With the naked eye small centers of degeneration may sometimes be noted in the gray matter, but often they may be sought for in vain.

4. The most constant lesions are microscopic in character; they are found more especially in the gray matter surrounding the cerebro-spinal canal and in the motor centers of the medulla and spinal cord. These lesions consist at first in hyperamia and accumulations of embryonic cells around the small vessels, perithelial or migratory in origin, often showing indirect division; finally there are also found lesions of nerve cells.

5. The lesion of the nervous elements of the parts indicated is quite characteristic ; it consists of signs of proliferation, namely, in the presence of several small cells in place of one large one, or in a uniform degeneration and often in the appearance of vacuoles with a reduction in size or disappearance of the nucleus, or, again, its chromatic network disappears. These cells frequently contain pigment. Round uninuclear, more rarely multinuclear, elements of a lymphatic origin often invade the protoplasm even of the cell and fill out 
the dilated pericellular lymphatic spaces by a multiplication of small nuclei.

6. The lesion of medullary substance is less pronounced, it consists chiefly of an oedema of the medullary sheath of the nerve fibers.

7. In certain plasma cells, in the interior of and around vessels, sometimes in leucocytes, in lymphatic spaces, in the altered parts of certain nerve cells, and in the dilated sheath of nerve fibers may be seen round or amœboid granules about I $\mu$ in diameter, pigmented or stainable by aniline dyes, and which in part seem to posses the power of movement.

More recently Babes has noted, besides the lesions above mentioned, that the alteration of the nerve cell is usually accompanied by a modification of their protoplasmic network and concludes that "Whilst admitting that the lesions of rabies are not absolutely characteristic, and that it may be that in a case of diffuse, very acute nuyelitis similar lesions may be found, it is necessary all the same to state, that neither in writing nor in my personal experience have I ever met with a similar case, so that at present we may consider the lesion of rabies as characteristic. In other infectious diseases there have also been found histological lesions characteristic as a whole, although composed of elements not absolutely specific."

Golgi draws attention to the following morbid changes in rabies :

(I) Changes in the structure of the nucleus, all the various phases of karyokinesis may be simulated, yet no true nuclear division may take place. (2) Changes in the body of the cells, such as vacuole formation, bladder-like transformation of the cells. Changes may also be recognized by methods directed to the study of the outer form of the cell. Here varicose appearances of the cell processes may be seen. Granular fatty changes may also be present. An important change lies in the displacement of the nucleus. The periphery of the cell becomes homogeneous. Granular fatty changes are also seen in the neuroglia cells. (3) Changes in the intervertebral ganglia. The author would look upon these anatomico-pathological changes found by him as characteristic, 
while here not only the sum total of the changes, but also their order of occurrence and mutual interdependence are taken into consideration.

The morbid process is a parenchymatous encephalo-myelitis, of which the exact exciting case is as yet unknown. The changes are thus grouped: (1) appearance of nuclear chromatin, peculiar cell division (neuroglia cells and vascular endothelium,) nuclear morements also in nerve cells, diffuse vascular distension and leucocyte infiltration, revealing a condition of irritation; (2) swelling, vacuolation, changes of form, granular appearance of nerve cells and neuroglia; and (3) more adranced changes in the nerve elements. The changes in the first group may be seen as early as five days after inoculation.

In a more recent article by Germano and Capobianco attention is called to the fact that the destruction of some of the nerve cells in rabid animals is not accepted by everybody, but that in their researches they have been able to confirm the statements made by Golgi, that instances of the complete disappearance of nerve cells have been observed, while other cells showing fatty degeneration and partial destruction of their entirety represent intermediate stages between the normal cell and its total disappearance. The alteration of the nucleus may precede or follow that of the cell body.

The nerve fibers, either in the white or gray matter, undergo a certain amount of change. In a longitudinal section of the myel, especially through the rentro-lateral columns, there are noted marked changes in the axis cylinders. In some cases they appear uniformly swollen for their whole length, while in others there are varicose enlargements. In the swollen portions there were frequently observed small vactoles which interrupted the continuity of the axis cylinder.

During the year 1900 , the discovery of changes distinctive of rabies was announced by Van Gehucten and Nélis. These changes are found in the peripheral ganglia of the cerebro spinal and sympathetic systems and are especially marked in the plexiform ganglion of the pneumogastric nerve and the gasserian ganglion. Normally these ganglia are composed of 
a supporting tissue holding in its meshes the nerve cells, each one of which is enclosed in a capsule, made up of a single layer of endothelial cells. The action of the rabic virus seems to exercise its effect on these cells particularly, bringing about an abundant multiplication of the cells forming this capsule, leading finally to the complete destruction of the normal ganglion cell and leaving in its place a collection of round cells. Ordinarily a considerable number of ganglion cells will be found which have undergone only a slight change, but under certain conditions the process is so widespread that all the ganglia cells are destroyed. The intensity of these changes varies in different animals; they are perhaps most pronounced in the dog, less marked in man and still less in the rabbit.

Much of the value of these findings consists in their making a sure and quick means of diagnosis. It is possible to complete the examination within six hours after the death of the animal, and under ordinary circumstances a positive opinion can be given in from 24 to 36 hours. It is important that the animal should be allowed to die and not be killed prematurely, as where the disease is not permitted to run its full conrse ending in death, the changes may be absent or only slightly developed.

$\$$ 242. Differential diagnosis. From the often obscure manner of infection, the long period of incubation, the variable symptoms and the absence of gross morbid changes characteristic of the disease, it is easy to mistake rabies for various other nervous disorders and vice versa, unless a definite method of diagnosis can be availed of.

Diagnosis by animal inoculation. The method which the experience of pathologists has shown to be the best, is the subdural inoculation of rabbits with a suspension of the brain or spinal cord of the suspected animal. The subdural inoculation with the brain tissue of rabid animals was first demonstrated by Pasteur to be more reliable and more rapid in its results than the subcutaneous injections. The procedure is simple. The brain of the suspected animal is removed with aseptic precautions as soon as possible after death. A small piece of the brain or spinal cord is placed in a sterile mortar aud thor- 
onghly ground with a few cubic centimeters of sterile water or bouillon. This forms the suspension to be injected.

The hands of the operator and all instruments are carefully disinfected. The rabbit is etherized, the hair clipped from the head between the eyes and ears, and the skin thoroughly washed and disinfected. A longitudinal incision is then made, the skin and subcutaneous tissue held back by means of a speculum, a crucial incision is made in the periosteum on one side of the median line, to avoid hemorrhage from the longitudinal sinus, and the four parts of the periosteum reflected or pushed back. By the aid of a trephine a small button of bone is easily removed leaving the dura mater exposed. With a hypodermic syringe a drop or more of the rabid brain suspension is injected beneath the dura, the periosteum is replaced, the skin carefully sutured and disinfected and the rabbit returned to its cage. As soon as the influence of the anæsthetic* has passed off the rabbit shows no appearance of discomfort. If the operation is performed in the forenoon the animal partakes of its evening meal with the usual relish. The inoculation wound heals rapidly and the rabbit exhibits every appearance of being in perfect health until the beginning of the specific symptoms, whicl occur ordinarily in from fifteen to thirty days after the inoculation. Occasionally the symptoms appear earlier than fifteen days and in some cases the rabbits are not attacked for from one to three months.

Symptoms. The symptoms following the inoculation are quite uniform. There is, however, a marked difference in the length of time the rabbits live after the initial manifestation of the disease. The fact should be clearly stated that rabbits do not ordinarily become furious. In some instances they are somewhat nervous for a day or two preceding the paralysis. There appears to be marked hyperæsthesia. Usually the first indication of the disease is a partial paralysis of one or both hind limbs. This gradually advances until the rabbit is com-

*Ether should be used in preference to chloroform for rabbits, as the latter frequently causes death, while the former can be administered with comparative safety. 
pletely prostrated, the only evidence of life being a slight respiratory movement. The head occupies different positions. In some it is drawn backward as in tetanus; in others it is drawn down with the nose near the fore legs and in still others it is extended as if the animal were sleeping. The period of this complete paralysis varies from a few hours to a few days, but ordinarily it has not exceeded twenty-four hours. Although these animals are unable to move voluntarily there is a reflex action of the limbs until a very short time before death.

During the period of incubation the temperature of the rabbits is normal. As the time approaches for the first symptoms to appear there is an elevation of temperature of from $I$ to 2 degrees, which continues for a variable length of time, but rarely longer than two days. This is followed by a gradual or usually a more rapid drop to the subnormal, which continues to the end.

The differential diagnosis in experimental animals is not difficult. Rabbits inoculated with several varieties of pathogenic bacteria frequently exhibit symptoms of paralysis for a brief period preceding death. In cases oi injury to the brain or spinal cord there may also be paralysis, which in the absence of the history of the case might be taken for that of rabies. In these cases, however, the symptoms appear very soon after inoculation. This is especially true when the paralysis is due to mechanical injury of the brain or to irritation or septic substances and in the case of the pathogenic bacteria if paralysis occurs at all it is almost invariably preceded by marked disability. This method of diagnosing rabies requires that the inoculated animals remain apparently well for a considerable length of time after the subdural inoculation and before the paralytic symptoms appear.

The lesions found on the post-morten examination are also of much assistance in making a diagnosis. If the animal died from septicæmia or brain injury there will be lesions almost invariably recognizable in the brain or viscera. In the case of septicæmia a bacteriological examination will reveal the presence of microorganisms. If the death was caused by 
rabies the inoculation wound in the head should be healed perfectly, there should be no abscess and the meninges should be free from exudates and the brain itself should appear perfectly normal, except that in rare cases there may be a slight injection of the blood ressels. The viscera are ordinarily normal in appearance, with possibly the exception of the liver, which we have frequently found to be deeply reddened and the gastric mucosa, which not infrequently shows dark patches, indications of disintegrated hemorrhagic areas. A bacteriological examination fails to reveal the presence of microorganisms in either the tissues or blood. Another important point which has been noticed is an intense rigor mortis following death fron rabies. Kinyoun states that this was a constant feature of this disease in all of the produced cases which have come under his observation. Wesbrook has not found this condition.

Animals other than rabbits have been used and a number of other methods of inoculation have been proposed.

Diagnosis by histological examination. The rapid diagnosis by means of the histological clianges pointed out by Van Gehucten and Nélis has been very successful in the experience of Ravenel. He states that: "The ganglion of the preumogastric nerve is selected by preference and should be removed as soon as possible after the death of the animal. It is put into absolute alcohol or ten per cent formalin solution. If put into absolute alcohol it should be removed to a fresh portion of alcohol at the end of six hours, in which it remains for six hours, when it may be transferred for one hour to a mixture of absolute alcohol and chloroform, and then put for one hour in pure chloroform, then for one hour in a mixture of chloroform and paraffin, and lastly imbedded in paraffin. If formalin is used for fixing, the ganglion should be removed at the end of six or eight hours, put for six hours more in 95 per cent alcohol and then into absolute alcohol for six hours, after which it may be fixed in blocks for cutting, by mucilage or gum arabic, or else imbedded in collodion. The characteristic changes of the capsule are brought about best by the use of hematoxylin, or hemalum and eosin. The method of Nissl 
gives good results also but as it requires some special technical knowledge to be efficiently carried out the former is recommended for its simplicity and ease of execution. This method has stood the test in Europe and America for nearly two years and although a large number of animals having different diseases have been examined with the object of determining whether or not these changes may occur in other conditions, they have never been found.

At the laboratory of the State Live Stock Sanitary Board, which was the first in this country to take up this method, fifty-two cases have been examined since May, I90o, without a single failure. These cases have occurred in mankind, dogs, cows, cats and rabbits, and the characteristic changes have been found in each of these species. We have not had an opportunity of examining horses, sheep or swine. In this laboratory it has replaced the slower and less certain method of inoculation almost entirely. Inoculations are now practiced only in those cases in which the material is sent in such condition that the microscopic examination is impossible."

$\$ 243$. Prevention and treatment. The prevention of rabies infection resolves itself into two procedures. (1) The destruction of all ownerless and vagrant dogs and (2) the muzzling of all dogs that appear upon the streets or in public places. In thus preventing the propagation of the virus as shown by the results obtained in Germany and Great Britain the disease will be practically exterminated.

There is no treatment for rabies except the preventive inoculation known as the Pasteur treatment by which an inmunity is produced by the subcutaneous injection of the virus of rabies in an attenuated form, beginning with the mildest virus and going gradually up to one which possesses nearly or full virulence. The attenuation of the virus is brought about by drying at a fixed temperature and the action of the atmosphere. Depending upon the length of time the virus is exposed to the influences, we can obtain any degree of virulence desired, the loss of virulence under fixed conditions being quite uniform.

The disease as seen in dogs infected naturally was called by Pasteur "street rabies" and the virus of such animals is 
known as the "virus of street rabies." Such virus will as a rule produce the disease in rabbits by intra-cranial inoculation in from three to four weeks. By inoculating rabbits in series one from the other, a reduction of the period of incubation is obtained. After about 100 passages rabbits will die with certainty and great regularity on the sixth or seventh day after inoculation. Beyond this point no increase of virulence has been obtained. This is the fixed virus of Pasteur.

\section{REFERENCES.}

1. BABES. Sur certains caracteres des lesions histologiques de la rage. Ann. de l'Institut Pasteur. Vol. VI. 209-223.

2. САВот. Report on experimental work on the dilution method of immunization from rabies. Jour. Experimental Med. Vol. IV. 1899.

3. DULLES. Disorders nistaken for hydrophobia. Trans. of the Med. Soc. of the State of Penn. 1884.

4. FLEMING. Rabies and hydrophobia.

5. KEIRLE. A report on the autopsies on four recent cases of rabies and a bacteriological examination of a rabid dog, together with the recent laboratory experiments. Maryland Med. Jour. Vol. XXXviII. No. I. 1897 .

6. LAw. Rabies. A System of Practical Hedicine by American Authors. Vol. III. ISgs.

7. MacClure. Rabies-hydrophobia. Supplement to the Annual Report of the Wichigan Board of Health. I 895 .

8. MOORE AND Fish. A report on rabies in Washington, D. C. Annual Report. U. S. Bureau of Animal Industry: 1895-6.

9. Public Health Commission, District of Columbia. Rabies. Bul. 25. U.S. Eureau of Animal Industry. 1900

io RAVENEL AND MICCARTHY. The rapid diagnosis of rabies. Univ. of. Penn. Med. Magazine January. Igor.

i . Ravenel. Rabies Bul.79. Dept. of Agr., State of Penn. igo1.

12. Sal,ion. Rabies, its cause, frequency and treatment. Year Book, Dept. of Agriculture. Washington, D. C. Igoo.

13. Salmon. Rabies in the District of Columbia. Circular 30 , U. S. Burcau of Animal Industry. 1900.

14. Suzor. Hydrophobia. An account of M. Pasteur's system. I887.

15. WESBROOK. Preliminary report on the laboratory diagnosis in twenty cases of suspected rabies. Trans. Am. Public Health Assn. S98.

\section{DIPHTHERIA IN FOWLS.}

- 244. Characterization. Diphtheria of birds is an infectious disease the lesions of which first appear on the mucous membrane of the nasal passages, the eyes, the mouth, the pharynx and larynx and which may extend to the trachea, bronchi, the air-sacs, the intestines and possibly to other 
abdominal organs. The disease is determined by a grayishyellow, fibrinous exudate which forms upon the mucous surface of the parts mentioned. The exudate may be so abundant as to obstruct the air passages. In some outbreaks, it is very acute, progresses with great rapidity and destroys most of the birds attacked. It is popularly known as "roup."s

Fowls (genus Gallus) and pigeons (genus Columba) are most commonly attacked and they are the only ones considered in this discussion. Avian diphtheria is reported, however, to attack turkeys, ducks, pea-fowls, pigeons and pheasants. It is presumed that wild birds may be affected.

Avian diphtheria is quite distinct from human diphtheria. There are cases on record, however, which indicate that the diphtheria of fowls may be communicated to children and cause a serious and even fatal sore throat. On the other hand, it is asserted that diphtheria of children is sometimes communicated to fowls and that the virus may be thus preserved for a considerable time and again be transmitted to children. Concerning this point additional investigations are needed.

$\$ 245$. History. The history of this disease is somewhat obscure. It is evident from the literature, that fowls have always been subject to various affections of the head but the first investigation of this class of maladies seems to have been made by Loeffler* in I88+. Since that time Klemmer, $\uparrow$ Babes and Puscarin, $₫$ Eberlin, || Loir and Ducloux and others have studied diseases known as diphtheria in pigeons, fowls and other birds. The disease was investigated by the Bureau of Animal Industry in $1893^{-4}$.

\$246. Etiology. In I884, Loeffler discovered a bacterium which he believed to be the specific cause of diphtheria in fowls and with which he could produce the disease. It

\%The origin of this term is somewhat obscure, but it is supposed to be a corruption of croup, and its application explained on account of a peculiar hoarseness accompanying the respiration of the affected birds.

"Mitt. aus dem Kaiserlichen Gesundheitsamte. Bd. II. (1S84), S. 2 I 4.

†Berliner thierärzt. Wochenschrift. I 890, No. IS, S. I3s.

$\doteqdot$ Zeitschrift f. Hygiene. Bd VIII. (I890), S. 374 .

Monatshefte f. Thierheilkunde. Bd. V. (r894), S. 433.

Ann. de l'Inst. Pasteur. Tome VIII. (I894), p. 599. 
differed from the diphtheria bacterium in man. Loir and Ducloux isolated a still different organism. The writer found in the exudates of the earlier stages of the disease a bacterium belonging to the septicaemia hemorrhagica group. It was rapidly fatal to rabbits but the diphtheretic lesions could not be produced by inoculation in fowls. There are marked differences in the different bacteria isolated and suspected of etiological significance. It is not positive, however that the same disease was studied by the different writers. There is little doubt of the specific nature of the disease. It is usually introduced into a flock by the exposure of the birds to sick ones at shows or by bringing affected birds on the premises. The contagion 111ay be carried by birds which have the disease in so mild a form that they show no symptoms of it. There is a general belief that the disease may be developed by exposure of birds to dranghts of air or by keeping them in damp, filthy and badly-rentilated houses. It is presumable that this belief in its etiology is not well founded because of confusion existing concerning the early symptoms of acute diphtheria and those of all stages of the chronic form and those of simple colds and catarrhs. Dampness and lack of ventilation no doubt favor the maintenance of the virus when introduced and predispose the birds to the disease. The specific cause of the disease known as diphtheria or roup in chickens and pigeons in the opinion of the writer is not known.

$\$$ 247. Symptoms. There is a watery secretion from the nostrils and often from the eyes, with general weakness and prostration greater than would be expected from simple catarrh. The birds sit with the back arched, the head and neck drawn down towards the body, the plumage roughened; the respiration is more or less obstructed, rapid and audible, the rision is impaired and swallowing is difficult. There is frequent shaking of the head, sneezing and expectoration of mucous secretions. If the mouth is examined at this early period the tongue is found to be pale, while small grayish spots, shaded with black and slightly projecting above the surface are seen along the border, the upper surface or at the base. 
The following day the condition is aggravated, the temperature is several degrees above normal, the appetite has disappeared and there is diarrhoea with greenish or yellowisl evacuations. From the open beak there escapes a thick, stringy, grayish mucus. The eyes are unnaturally dilated, projecting and possibly partly covered with the thick secretion which has accumulated between the lids. The nostrils are obstructed by the thickened and dried secretion. Walking is irregular and difficult. The mucous membrane of the mouth and pharynx is congested and shows numerous dark red elevations covered with fibrinous exudate. The patches on the tongue have increased in size, they are gray in color, dried along the edges of the tongue but soft and flattened upon its upper surface. They are covered with membranous deposits. The voice often fails.

$\$ 248$. Morbid anatomy. The lesions are largely localized on the mucosa of the head. With the exception of emaciation, there are no lesions or evidence of organic disease. The cause of death and the extreme emaciation is difficult to explain in those cases where the lesions were confined to one eye or to the mucosa of the nares, excepting on the supposition that some poisonous or toxic substance was absorbed from the seat of the disease. In those cases where the lesions are in both eyes, or in the mouth and throat, difficulty in finding or swallowing food affords a rational explanation.

In some cases the exudate is of a croupous character, in others of a diphtheritic nature. Three stages or varieties of lesions which represent the types of this disease as encountered in this country may be more definitely defined as follows.

(I) An exudate of a serous or muco-purulent character in the conjunctiva and nasal cavities. Ordinarily this condition cannot be recognized in the mouth. The mucosa in these cases are apparently but slightly altered.

(2) The mucosa over a small or larger area is covered with a spreading exudate of a grayish or yellow color. It is firmly attached to the mucous membrane and when removed leaves a raw, bleeding surface. Sections through this exudate and the subjacent tissues show that the epithelial layer is des- 


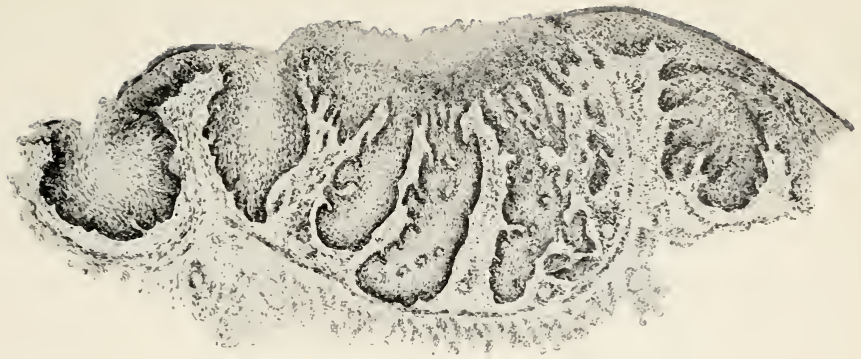

I.

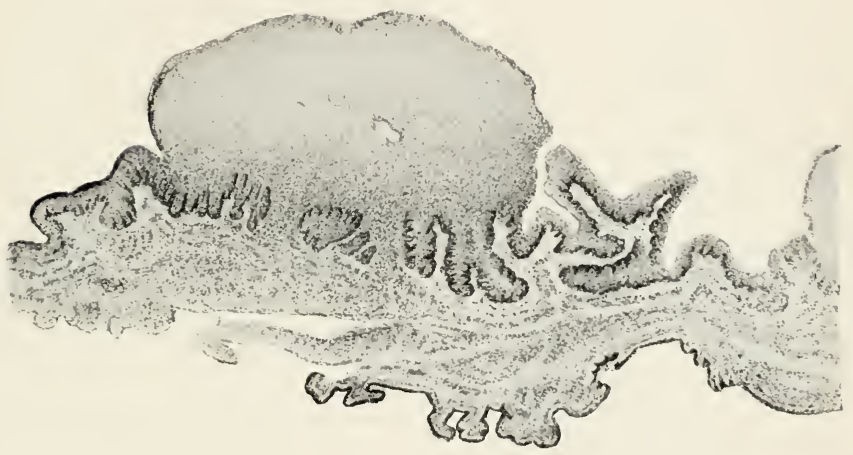

2.

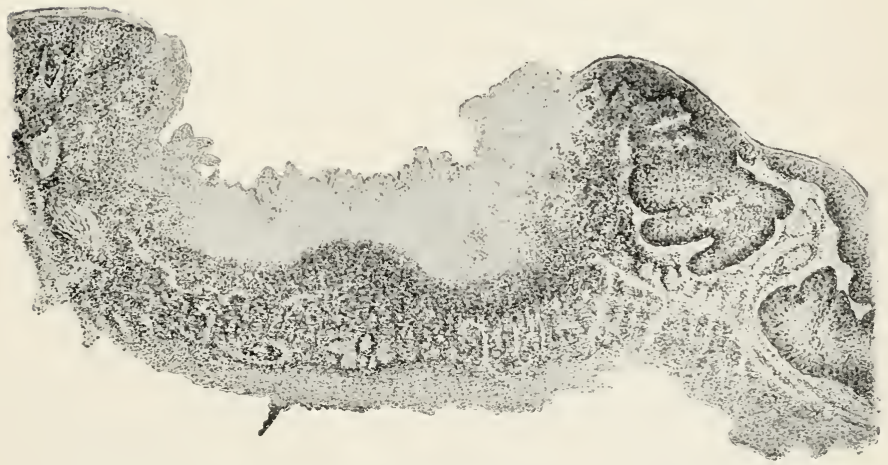



troyed and the underlying tissue infiltrated with cells. The extent of the infiltration varies in different individuals.

(3) The mucosa is covered with a thick mass of exudate, varying in color from a milky white to a lemon yellow or brown. It is easily removed, leaving a more or less granular and healed surface. This sloughed mass is frequently dried at its margins to the adjacent tissue. It enits a strong putrid odor, due to decomposition. The drying of the margins prevents the fowl from expelling the exudate after it becomes separated from the underlying tissue.

The evidence to support the supposition that the three forms or types of exudate described are different stages in the same morbid process, as gathered from the post-mortem notes and bacteriological study of the cases investigated, may be summarized as follows.

(a) Abnormal conditions, representing the intermediate and connecting links between the types of lesions, are frequently encountered.

(b) Although at the time of examination (post-mortem) but one form of exudate is usually present in a single fowl, there are exceptions, in which two and occasionally the three forms are coincident. Thus the eye is covered with a sloughed exudate, the posterior nares contains a layer of muco-purulent substance and on the mucosa of the mouth are areas of a diphtheritic exudate.

In fowls which die, the exudates are for the greater part in the advanced stage, although fatal cases occur in which the lesions are restricted to an abnormal quantity of a serous or muco-purulent, more or less viscid, exudate in the conjunctiva or nasal cavities. The best illustration of the diphtheretic process is found in fowls killed for examination in the second stage of the disease. The distribution of the lesions shows that the conjunctiva is most frequently affected. The exudate in the nasal cavities is in some cases undoubtedly the result of the coagulation of the liquid which has passed during the course of the first stage from the conjunctiva through the lachrymal duct into the nares. In certain cases, however, the lesions appear in the nares primarily. Sections of the exudate, with subjacent 
tissue from the cornea and the mouth, show that there is a cell infiltration into the mucosa which destroys the epithelial layer and frequently the submucous tissues to a considerable depth. See Plate VIII.

The fact should not be overlooked that the disease in the eye is usually confined to the conjunctiva and the cornea, the posterior portion remaining apparently normal.

From the observations thus far made the provisional theory is entertained that the three forms of the exudateserous or muco-purulent, diphtheritic and sloughed massrepresent three stages in the course of the same disease. It is easily understood that fowls examined in the first stage would be said to be affected with a catarrhal condition of the mucosa of the eyes or nares. It is highly probable that in many cases the disease never reaches the second stage and if these cases alone were examined the diphtheritic condition would not be suspected. It appears, however, that in the majority of cases the disease runs its course and membranes are formed, slough and recovery follows. It is further presumable that the disease in question appears sometimes in a virulent and destructive form. I am in possession of statements from poultry raisers which show that there are occasionally epizcötics of a disease characterized by exudates in the eyes, nose or mouth, which runs a rapidly fatal course. It appears that it is sucli outbreaks which have been reported in Europe as diphtheria and not the low form of chronic disease which has been studied in this country.

$\$ 249$. Relation of diphtheria in man to that in fowls. A comparison of the hacillus of diphtheria in man (KlebsLoeffler) with those described from diplitheria in fowls, shows that norphologically and in their pathogenesis for experimental animals the organisms are in no way alike. There is also a marked difference in the nature of the exudate in fowls and in man. The non-identity of these diseases has been clearly pointed out by Ménard*. Although these maladies are shown by several observations to be unlike in their etiology and the character of their lesions, the transulission of fowl

*Revue d'Hygiene. Tome XII. (IS90), p. 410. 
diphtheria to the human species, and vice versa, is affirmed by several writers.

Gerhardt + reports + cases of diphtheria in Wesselhausen, Baden, among 6 workmen who had charge of several thousand fowls, many of which died of diphtheria. There were no other cases of diphtheria in the neighborhood and the evidence was quite conclusive that the disease was contracted from the affected fowls. It is also stated that an island on the northeastern coast of Greece had been free from diphtheria for at least a third of a century, when a dozen turkeys, several of which were diseased, were introduced. Soon afterwards diphtheria appeared in a house near the garden where the turkeys were kept. The disease became epidemic on the island causing the death of 36 people, over 40 per cent of those attacked.

Debrie reports briefly the clinical history of 6 cases of diphtheria which occurred in the garrison of Sebdou, and states that while the sixth case ( 2 were fatal) was still under treatment in the hospital ro fowls kept in a house not far from the hospital were attacked with diphtheria and exhibited symptoms strikingly like those present in the human beings. Five of the ro fowls died and two heads were sent to Arloing, who confirmed the diagnosis of fowl diphtheria. The fowls were fed by a hospital attendant and it was ascertained that an identical outbreak had occurred among the fowls at a neighboring place from which one of the 6 cases of human diphtheria had been brought. Debrie is inclined to the view that human diphtheria is transmissible to fowls and fowl diphtheria to man.

Cole* reports a case near Jacksonville, I11., where a flock of fowls became affected with a disease characterized by an exudate on the mucosa of the head. Some of the exudates emitted a foul odor. As the weather was cold, one of the chickens was taken into the house where a child about $2 \frac{1}{2}$ years old fondled it. Four days later the child was taken sick apparently with diphtheria, from which it died. There were no other cases in the neighborhood and the affected chicken was the only possible source of infection.

$\nmid$ Revue f. Thierheilkunde u. Viehzucht. Bd. VI. (IS83), p. ISo.

${ }_{+}$Reviewed in Centralblatt f. Bakteriologie. Bd XIII. (I893), S. 730.

*Archives of Pediatrics, XI, ( IS94), p. $3^{81}$. 
The diphtheritic disease of fowls reported by Loir and Ducloux in Tunis, in 1894 , spread to the people of that place, resulting in an epidemic of serious proportions. Ménard refers to the fact that men employed to feed young squabs contracted diphtheria by blowing the masticated food into the mouth and crop of squabs suffering with that disease. Schrevens $\ddagger$ reports several cases of diphtheria in children in which he traces the source of infection to certain poultry.

Although the number of reported cases of the transmission of fowl diphtheria to the human species and vice versa, is small in comparison with the extent of the disease in poultry, the evidence that such a transmission is possible is quite sufficient to discourage the careless handling of diseased fowls. It is a quite common practice, especially in the rural districts, to bring the sick chickens into the louse for treatment, where the children of the household are allowed to fondle them at will. It is not improbable that when this disease is thoroughly investigated the number of cases of direct infection from this source will be found to be much larger than it is at present supposed. Until such investigations are satisfactorily completed the indiscriminate handling of diphtheritic chickens especially by children and the exposure of fowls to the infection of diplitheria in the human species, whereby they may becone carriers of the virus, should be strenuously avoided.

$\$ 250$. Prevention In order to prevent this disease it is evident that many conditions must be strictly complied with. The character of the food and the general sanitary conditions, including cleanliness, ventilation and the temperature of the poultry houses must be considered. Undoubtedly there is much to be learned in connection with the proper care of poultry. In addition to the general sanitary methods, the following rules should be observed.

(I) Fowls which have an exudate on any of the mucous membranes of the head or which have come from flocks in which such a disease exists or has recently existed, should not be placed among healthy poultry.

$\ddagger$ Bulletin de l’Acad. Royale de Méd. de Belgique, VIII. (IS94), p. 38 o. 
(2) If the disease appears in one or more fowls of a flock they should be immediately separated from the well ones. If possible, the source of the infection should be determined and removed.

(3) The quite common practice of allowing fowls from different flocks to run together during the day should be discouraged.

(4) Care should be taken to avoid the possibility of bringing the virus of the disease from affected flocks in the dirt or excrement which naturally adheres to the shoes in walking through an infected chicken yard. The same care is necessary in the interchange of working implements, such as shovels, hoes and the like.

It is evident to any careful observer that the fact is too often overlooked that fowls, owing to their method of living, are more liable to infection than other farm animals. This is especially true when they are allowed to run at random, as they too frequently are, picking their living from the garbage pile and barnyards, or securing even more unwholesome food. There is little doubt that many so-called outbreaks of contagious disease among fowls are simply enzoötics brought about by improper care.

The wide distribution, the large number of fowls affected and the usual chronic course of this disease render it one of the few poultry affections for which curative measures promise to be of practical value. Although prevention is the safest of cures, when the disease is once introduced as it is in a very large number of flocks, the necessity for remedial treatment is apparent and where economy is to be considered should be recommended. The practice sometimes followed of destroying all of the affected birds should be discouraged. Although experiments have not been made to test the efficiency of remedies already recommended or to investigate the practicability of others, the testimony of many practical poultry raisers is to the effect that the disease is amenable to treatment. The most certain of the known methods of treatment is the local application of disinfectants, among which a weak solution of carbolic acid appears to be the most satisfactory. 
The fact that the lesions are so much exposed renders the disease especially favorable for topical applications.

DESCRIPTION OF PLATE VIII.

1. Early stage of membrane formation in the throat of a pigeon.

2. A section from an adranced stage in which the mucosa is cozered with a thick grayish necrotic exudate.

3. A later stage where the exudate has sloughed.

REFERENCES.

I. MOORE. A preliminary investigation of diphtheria in fowls. Bulletin No. 8. U. S. Bureau of Animal Industry. 1895.

2. SALMON. The diseases of poultry. IS99. p. 216.

\section{INFLUENZA.}

Synonyms. Epizoötic catarrhal fever ; epizoötic catarrh; horse distemper ; pink eye; monntain fever ; shipping fever.

$\$ 251$. Characterization. Influenza is an acute infectious disease characterized by a rise of temperature and a catarrhal condition of one or more of the mucous membranes, more especially of the head. One or more of the internal organs may become affected. It usually appears in epizoötic form. It is a disease of horses although asses and mules are susceptible and a few cases are reported of its being transmitted to man and to dogs.

Influenza is a generic term employed to designate a large variety of symptoms. A somewhat careful analysis of its manifestations suggests that possibly it includes a number of etiologically distinct diseases $i$. e. morbid conditions brought about by different causative factors. The term has long been employed to designate a considerable variety of equine epizoötics the independence of which could not be established. The disease, as it is seen in the horse, suggests further that possibly it is in its beginning, a general affection because of the early rise of temperature and that later in its course it becomes, to a limited extent, localized. At present, influenza is restricted to groups of symptoms and lesions in the horse that are not 
very unlike those of la-grippe in man. Its symptoms, lesions and sequelae warrant such a view at least for a working hypothesis. As it is not usually fatal, little progress seents to be made in acquiring knowledge concerning the nature of its morbid anatomy. There is much need for careful investigation of this very common malady.

$\leqslant$ 252. History. According to the writings of Falke, influenza was recognized in very early times. There is evidence that it was known in the fourth and fifth centuries. It was described by Löw in 1729 as an equine epizoötic which had spread over Southern Europe. It is also stated that cases of its having been transmitted to man had occurred. Gibson observed it in 1872 in London and in different districts of England. It raged in epizoötics in 1760,1776 and 1803 . The disease was widely disseminated during the last century. The more important epizoötics are reported in the years from 1813 to 1815,1825 to 1827,1836 to $1840,1846,1851,1853,1862$, I870, 1873, I88 1 to 1883 , and 1890 . Anker who described influenza in Switzerland in $1 \&_{2} 6$ laid stress on its contagious nature and stated that in his opinion "a rolatile infectious matter was the cause."

Influenza spread as an epizoötic in $18 ; 2$ to 1873 over the greater part of the United States where it received the name of "pink eye" (French, Fievre typhoide). It started in Canada and extended south and west reaching into British Columbia to the north and Mexico at the south. The last great epizoötic raged in Europe from $188 \mathrm{I}$ to 1883 during which time it is said to have spread over nearly the whole continent. In the Prussian army, 8, 434 horses became infected in $1890 ; 2,497$ in I 89 I ; and 3,645 in I892. In Copenhagen, 3, ooo horses suffered from it in 1890 and 1891 .

$\$ 253$. Geographical distribution. Influenza seems to be known in nearly if not all of the countries of Europe and America. In certain sections of the United States it is almost a constant affection. This is especially true of certain cities owing to the constant introduction of "green" horses.

$\$$ 254. Etiology. Influenza seems to be produced by a specific infection the nature of which has not yet been deter- 
mined. It spreads rapidly among horses. The virus appears to lose its virulence quickly outside of the animal body, but within the body it sems to be preserved for a long time. According to the observation of Jensen and Clark, stallions which have had the disease may transmit it, to the mares they serve, for months after apparent recovery. Dieckerhoff succeeded in transmitting the disease to healthy animals by subcutaneous and intra-renous injections of the blood of infected horses, but Friedberger and Arloing failed to do so. Horses are most susceptible. Sex, breed, stable management and feeding appears to have little or no influence on their individual susceptibility.

Infection usually takes place from horse to horse. The virus appears to be carried by infected human beings, litter, harnesses and thermometers. In many cases one attack confers immunity but a second infection or a relapse frequently occurs. Toward the end of an epizoötic the disease is usually milder in form, probably due to a gradual attenuation of the virus.

$\$ 255$. Symptoms. The period of incubation varies from four to seren days after which the disease appears suddenly, and may attain its highest point of intensity within twenty-four hours. The organs of circulation, nervous centers, digestive and respiratory mucous membranes and conjunctiva are especially affected. There is partial or entire loss of appetite and depression. The temperature is characterized by a sudden rise of from 3 to $4^{\circ} \mathrm{F}$. or even more. It remains high with but slight variations for from three to six days and then falls rather quickly, often within twenty-four hours, to the normal. At first the frequency of the pulse is but little increased in comparison to the elevation of the temperature, but later it rises to from 60 to 70 and in fatal cases from 80 to soo or more. It generally continues high for some time even after the temperature has fallen. The fever is characterized by unevenness in distribution of the external temperature of the body. The early rise of temperature, while the affected horses appear to be healthy, is of much diagnostic value. 
Usually the nervous depression co-exists with the ferer. The animal may hold its head down and appear to be comatosed. Extreme muscular debility is frequently associated with this stupor. Tremors may occur, the hind legs may give way while walking and paralysis of the hind quarters appears in a few cases.

The oral mucous membrane is greatly congested, liot, dry or coverad with mucus. There is sometimes difficulty in swallowing. The aninal frequently yawns. There is usually constipation in the beginning of the disease which may be accompanied with colic. The feces are formed into small hard balls and are corered with masses of mucus. Later, diarrhoea with considerable tenesmus usually occurs. The feces are of a thin, pulpy, and even fluid consistence, and sometimes have a foetid odor. At the beginning of the attack the urine is alkaline but it becomes acid when the intestinal lesions are developed. It rarely contains albumin but desquamated $\epsilon$ pithelial cells of the bladder are often present in large quantity. A serere affection of the eyes is quite constant and quite a characteristic symptom of influenza. At first it consists chiefly of catarrhal and later of phlegmonous.conjunctivitis with considerable swelling of the eyelids which may be followed by keratitis and possibly by an exudative or hemorrhagic iritis. Usually both eyes are affected. The first indications are tears, intolerance of light, intense hyperemia of the conjunctiva and contraction of the pupil. The eyelids swell, are hot, painful and kept more or less continually closed. A grey, mucopurulent secretion accumulates between the eyeball and the eyelids and the eyeball becomes very sensitive to pressure. The cornea at the beginning of the keratitis has a greasy lustre, is at first iridescent, later on exhibits milky opacity over its surface. It is considerably injected with blood at its edge; the iris becomes swollen and yellowish in color. Often these inflammatory changes of the eye disappear in a strikingly short time.

During the further progress of the disease, swellings appear on the extremeties, sheath, epigastrium and lower part of the chest. It may be concluded that these swellings are 
due to oedema caused by passive congestion. Less frequentiy the swellings are of an inflammatory nature. The swelling of the extremeties causes the gait to be stiff and unwieldy. Inflammation of the sheaths of the tendons is sometimes observed.

The respiratory mucosa are congested. At first there is a serous and, later on, a muco-purulent discharge from the nose, slight swelling of the submaxillary glands, moderate acceleration of respiration and a cough. As a rule the animal becomes emaciated during the course of the disease, pregnant mares may abort.

The average duration of the disease is from six to ten days, although severe cases may run for two or three weeks and very mild cases may recorer in from three to six days.

In certain cases complications may arise such as cardiac debility, grave cerebral symptoms, severe gastro-intestinal inflammation, laminitis and petechial fever all of which have been described as accompanying complications.

The mortality varies at different times and in different places. The average appears to be from 0.5 to 4 per cent. Dieckerhoff saw a loss of + per cent among 1,700 horses; Aureggio, one of 3 per cent among Soo horses; Friedberger, one of 9 per cent; and Siedamgrotzky, one of ro per cent. It is stated that in 1872 , in Philadelphia, 7 per cent of 30,000 infected horses died.

$\$ 256$. Morbid anatomy. The principal tissue changes of influenza are met with in the organs of digestion. The mucous membrane of the pyloric portion of the stomach and of the intestines is hyperaemic, swollen and sprinkled with slight hemorrhages. The submucosa is yellowish in color and infiltrated with a gelatinous substance causing the membrane to form thick somewhat translucent elevations containing a fluid which coagulates. Peyer's patches are enlarged especially those in the neighhorhood of the ileo-caecal valve. The mucous membrane of the mouth and sometimes that of the pharynx show similar changes.

The mucous membrane of the upper air passages is hyperaemic and swollen. In rare cases, the mucosa of the 
larynx is inflamed. Also the subcutis when inflammatory swellings appear on the skin. Schïtz found that in the brain and spinal cord the arachnoid spaces are filled with a fluid which is generally clear although it may contain leucocytes. He reports one case in which the lateral ventricles contained a large quantity (20 c. c.) of fluid. The other lesions which may be found depend upon the extent of localization of the disease. Usually there are slight swelling of the spleen, small hemorrhages in the intestines, under the serons membranes and in the lungs, eyes and brain; gelatinous infiltration of the renal connective tissue and mesentery ; swelling of the lymph glands; yellowish serous exudates in the larger cavities of the body and imperfect coagulation of the blood. One or all of these changes may appear in a single animal.

$\leqslant$ 257. Differential diagnosis. Influenza must be differentiated from strangles and contagious pneumonia. The differentiation between pleuro-pneumonia and influenza is difficult only at the beginuing, when merely general symptoms, such as fever, loss of appetite and weakness are present. It should be mentioned that the two diseases may affect the horse simultaneously. When the skin is greatly swollen, influenza may resemble petechial fever, from which it can, however, very soon be distinguished by the absence of petechiae, by the mildness of its course and its greater contagiousness. In case of strangles, the lesions in the lymphatics may serve as distinguishing characters. If abscesses are in evidence the finding of the streptococcus of strangles would be quite conclusive. It would be positive providing Streptococcus equi could readily be distinguished from the pyogenic streptococci.

With these diseases, a diagnosis, save in the more typical forms, is difficult. Without a definite, recognizable, etiological factor or other exact tests, a positive diagnosis in doubtful cases can not be made.

\section{REFERENCES.}

I. LIGNIÈres. Influenza or infectious pneumonia, the etiology of equine. Jour. of Comp. Path and Therap. Vol. XI. (ISg8). p. 312.

2. M'FADYEAN. Influenza in the horse, what is it? Jour. of Comp. Path and Theraputics. Vol. II. (I889). p. Io5. 
3. MARSDEN. Influenza. The leterinary Journal. New Series. Vol. II. (I900). p. 315. (M. describes three forms (I) catarrhal fever, (2) bilious fever, (3) epizoötic cullulitis).

4. Nelson. Influenza. Bulletin 22. State Agric. Exper. Station, Washington. I 896.

\section{DOG DISTEMIPER.}

Synonyms. Dog plague; dog disease; bench show disease ; typhus fever in the dog; typhoid fever in the dog; febris catarrhalis epizoötica canum.

$\$ 258$. Characterization. Distemper is an infectious disease appearing in sporadic cases or in epizoötics. It is usually determined by a rise of temperature, loss of appetite and lassitude, followed by a catarrah of the conjunctiva, respiratory passages and digestive tract.. Frequently there are serious disturbances of the nervous system. It is the most important canine disease. It is reported that cats, wolves, foxes, jackalls, hyæenas and monkeys suffer from it.

\$250. History. This disease of dogs was known in quite early times. Its history shows that possibly it was introduced into Southern Europe from Peru, South America, about the middle of the eighteenth century. It was regarded as being closely allied to a number of diseases of the human species such as the plague and typhus. Trasbot believed it to be closely allied to, if not identical with, small pox.

\$ 26o. Geographical distribution. Distemper in dogs is a wide spread disease. It is exceedingly common in the United States and its ravages extend throughout America and seem to be no less in Europe. It is stated that there is no country or climate in which the dog is exempt from distemper.

$\$ 26$. Etiology. The specific cause of distemper has not been demonstrated. A large number and variety of bacteria have been thought by different investigators to stand in a casual relation to this disease. Schantyr stated, in 1892 , that canine distemper should be divided into three different diseases and that each is produced by a distinct species of bacteria. More recently (I899) Jess has isolated a bacillus from the catarrhal secretions, blood, serous exudates and 
organs. He reports having reproduced the disease in dogs with pure cultures of this organism. The writer has not been able to find this bacillus in a few cases which he has examined, but a streptococcus has often appeared in pure cultures from the various organs. There is much need for further investigation into the etiology of this malady. That it is produced by a specific cause is very clearly indicated by the reported results of investigations to the effect that dogs inoculated with the nasal discharge of affected animals derelop the disease.

$\$ 262$. Symptoms. The symptoms appear after a period of incubation of from four to six, possibly eight days. They vary to such a degree that it is impossible to refer to all of the manifestations. In some cases the symptoms suggest a general disorder. In others they are referable to certain parts or organs such as the mucosa of the digestive and respiratory tracts, the brain or integument. As a rule several organs are implicated.

The initial symptoms such as depression, roughened condition of the coat, loss of appetite and elevation of temperature are usually suggestive of a general disturbance.

In a large majority of cases, conjunctivitis is the primary ocular symptom. Tears flow from the eyes and photophobia is present. The mucous membrane of the eyelids becomes highly congested and swollen and the eyelids turgid. The discharge from the eyes which is at first serous soon becomes mucous and purulent. The exuded matter consists of pasty mucous or dirty yellowish pus. This exudate collects under the lower eyelids, chiefly at the inner canthus of the eye and soils the edges of the eye lids, upon which it frequently dries into crusts and causes the lids to adhere, especially during the night. Ulcers form on the cornea in consequence of the action of the accumulated and decomposing pus and the patient wiping and rubbing the eyes with its paws. The epithelium of the cornea sometimes suffers more or less from shallow flat lesions which give the surface of a cornea a rough and uneven appearance. Frequently smaller and deeper ulcers form especially toward the center of the cornea.

In other cases, there is a diffuse, parenchymatous keratitis 
which renders the cornea, to a considerable extent, opaque and gives it the appearance of ground glass. These extensive opacities sometimes develop in a comparatively short time. This affection of the cornea, the so called "distemper of the eyes" is frequently the only evidence of distemper with the exception of the high temperature.

There may be vomiting, well marked congestion and dryness of the oral mucosa. There is usually constipation at first, but later a diarrhoea in which the feces, as a rule, are very foetid, often slimy and frothy. Hemorrhagic intestinal catarrah sometimes exists. The urine frequently contains albumin, especially when the patient is weak or in an advanced stage of the disease, biliary pigments but rarely hemoglobin.

The nasal discharge is serous at first, mucous or purulent later on. It is followed by sneezing, panting and nasal pruritis, which causes the animal to rub its nose with its paws. The pus discharge from both nostrils may be very copious. It is sometimes mixed with streaks of blood, and varies in color from a dirty yellow to a dirty green. Later, it may become foetid and even watery. Ulcers may appear on the nasal mucosa.

Laryngeal catarrh usually accompanies the nasal catarrh and manifests itself by a congh, which comes on in paroxysms and which is at first hoarse and dry, but later moist and accompanied by a discharge of phlegm. The congh excites vomiting. The catarrh spreads from the larynx to the trachea and bronchi. The resulting bronchitis is followed by an increased rate of breathing and manifests itself by a cough and hoarse, sharp, resicular, respiratory murmurs. Frequently there is a catarrh of the mucous membrane of the smaller bronchi. There may be difficulty in breathing and a feeble cough which the patients try to suppress. The congh may be excited by percussion of the thoracic walls, by the animals getting up and by their being taken out of their kennels. Young and weak animals that cannot remove the accumulated phlegm from the bronchi by coughing beconse affected with catarrhal pneumonia. The pneunonia can be recognized by the increase in temperature.

Distemper often begins, especially in anaemic animals, with 
great depression and dullness. Strong animals, however, more usually exhibit symptoms of acute hyperemia of the brain, such as excitement, restlessness, yelping and even attacks of fury, which give way later on to manifestations of cerebral pressure. Spasms frequently occur either generally or confined to particular limbs which swing backward and forward as if affected by chorea. The animal may fall down as if suffering from epilepsy, bark, become unconscious and exhibit spasms of the muscles generally. The sphincters of the anus and bladder become relaxed and consequently feces and urine are involuntarily passed. There is a gradual return of conscionsness which, in a short time, is complete and the dog manages to get up although he is very weak. Such an epileptiform attack may pass directly into long continued coma.

Paralysis may follow the convulsions or it may come on simultaneously with them. It seldom occurs at the beginning of the disease. It may be confined to certain groups of muscles as those of a limb, the whole of the hind quarters or eren the entire body in the form of paresis, especially of the motor nerves, combined with excessive muscular weakness. The sick dog staggers and his hind quarters sway from side to side or he becomes incapable of supporting himself on his hind legs. Frequently he knuckles on all four legs and in severe cases is unable to stand. Permanent paresis of the hind quarters with paralysis of the bladder and rectum is a frequent result of distemper. In some cases there may be paralysis of the muscles of the tongue.

A characteristic pustular exanthema is frequently observed on the inner surface of the thighs and abdomen and in abortive cases it may be the only symptom of distemper. It first appears in the form of minute red spots, which after twenty-four hours develop into miliary nodules that are surrounded by a red ring. These nodules change into resicles and pustules which may become as large as a pea or bean. They dry into a yellowish brown crust or burst leaving a raw surface. Healing takes place with desquamation of the epidermis after about eight days leaving pigmented, pale reddish areas which persist for some time. Generally, there are only a few pus- 
tules present. The exanthema may spread, in the form of a scabby eczema, over the whole body, to the membrane of the external auditory meatus and less frequently to the mucous membrane of the mouth and eyes. This eruption, contrary to that of sarcoptic mange, is accompanied by only slight pruritis. Intense catarrh of the prepuce may appear simultaneously with the skin eruption.

The temperature which is usually very irregular, is higher during the initial stage than when local manifestations appear. It often falls with remarkable rapidity below normal towards the approach of death. If the disease runs a protracted course, the patient becomes emaciated, the hair loses its lustre, the body exhalations have a very foetid odor, the eyes are sunken, the mucous membranes become pale and the patient grows weaker, staggers when walking or lies in a state of coma.

In abortive cases recovery may take place in eight or ten days, although the disease usually lasts three or four weeks. With severe complications, especially those of the nervous system, distemper assumes a protracted course and is frequently. followed by sequelae, such as paralysis and convulsions, which may persist for months and even longer. The average mortality appears to be from 50 to 60 per cent.

$\$ 263$. Morbid anatomy. The anatomical changes in the respiratory system are those of rhinitis, laryngitis, bronchitis and catarrhal pneumonia. The nasal mucous membrane is either very pale or greatly congested, swollen and covered with a thick, purulent, greyish green or dull reddish inflammatory exudate which is mixed with coagulated blood and collects chiefly between the lamellae of the turbinate bones and in the frontal sinuses. Hemorrhagic ulcers are sometimes present. The mucous membrane of the laryux and bronchi is hyperæmic, swollen, often infiltrated with hemorrhages and covered with pus. Sometimes cattarrlal ulcers appear. The large bronchial tubes often fail to exhibit changes which might have been expected from the symptoms. The smaller bronchi are, on the other hand, frequently filled with dirty grey and even bloody, viscid pus. There are areas. of congestion on the surface of the lungs, some parts of which 
may contain but little air. There may be areas of collapse or those abnormally filled with air. The inflanmatory foci of the lungs are usually consolidated. In very young animals there may be a fibrous exudate which is very soft and which readily liquifies. The hepatization frequently involves an entire lobe. The hepatized parts are frequently studded with small suppurating foci, or are diffusely infiltrated with pus. The plura over the affected parts is often inflamed. The bronchial glands are swollen or infiltrated with a serous fluid or with pus.

In the digestive system, the mucous membrane of the stonlach and intestines, especially that of the small intestine, is hyperemic and swollen. It may be covered with a tough mucus and often sprinkled with hemorrhages. In other cases it is very pale, swollen and easily torn. Frequently the contents of the intestine are blood stained and the mesenteric glands enlarged and cedematons.

The brain is anaemic and often there is a serous effusion into the lateral ventricles and subarachnoid spaces. In a few cases there are signs of a purely venous, cerebral hyperemia as for instance, great congestion of all the sinuses, venous plexuses and vessels of the pia and the appearance on the cut surfaces of the brain of numerous blood points which can easily be wiped off. Kolesnikoff found microscopically the brain substance, especially the walls of the ressels, infiltrated with leucocytes. Krajewski noticed dilation of the ressels, cellular infiltration of their walls, filling of the perivascular spaces with lymphoid cells and migration of lymphoid cells into the stronia of the brain and into the protoplasm of the ganglionic cells. The changes in the spinal cord, which are not well marked, consist chiefly of anaemia and slight oedema especially in the lumbar region. Mazulewitsch states that in acute paralysis there are changes in the walls of ressels with an exudate along the vessels and in the interstitial tissue of the gray matter of the spinal cord. In chronic distemper, there is a localized interstitial myelitis with partial atrophy of the cord. Hadden found groups of emigrated blood corpuscles in it. In severe cases, according to Trasbot, the spinal cord and its membranes 
are often considerably injected with a sero-fibrinous exudate in and under the arachnoid and even into the substance of the spinal cord.

Among the other changes which have been described we may mention decrease in the total quantity of the blood of the body, combined with a certain degree of hydraemia. There may be fatty degeneration of the liver and kidneys. The muscular tissue of the heart is discolored in consequence of cloudy swelling and fatty degeneration of its fibers. The lymph glands may be oedematous.

$\$$ 264. Differential diagnosis. Distemper is to be differentiated from, (1) A simple primary catarrah, such as that of the eyes, nose, lungs, stomach or intestines. While the differentiation is often difficult at first, as distemper frequently manifests itself in the following affections, a single mucous membrane or in one organ. In general the diagnosis is obtained from the epizoötic nature of the disease, age of the patient, high temperature, simultaneous implication of several organs and the unfavorable course of the malady. The finding of the pustules of distemper is of much diagnostic value. (2) Rabies. The symptoms of cerebral irritation which occur at the beginning of the disease may give rise to the suspicion of rabies. The characteristic aggressive behavior of animals suffering from rabies is, however, absent in cases of distemper. The further course of the disease soon enables a diagnosis to be made. (3) The eruption of distemper if widely distributed over the body may resemble that of mange. The mild character of the pruritus, the presence of pustules on the hypogastrium and inner surface of the thighs, the rapid spreading of the exanthema over the whole body and the development of other symptoms of distemper, render the differentiation easy. The exanthema of distemper and that of mange, however, often occur simultaneously in the same animal in which cases certain precautions are necessary in making the correct diagnosis. (4) Epilepsy. The epileptiform attacks in distemper are distinguished from true epilepsy essentially by their being less acute. (5) It must also be differentiated from simple coryza, bronchitis, chorea and paralysis.

Until the specific cause is found and can be arailed of in 
making the diagnosis, much doubt will necessarily exist respecting the nature of the disease where inany of the symptoms and lesions are atypical.

$\$$ 265. Prevention. The lack of knowledge concerning the specific cause has rendered it impossible thus far to close all channels of infection but its spreading can be checked to a considerable degree by isolation and the use of disinfectants. It has been stated that the inoculation with the virus from very mild cases, which produces a mild attack, will immunize an animal for several years.

\section{REFERENCES.}

I. HeRtwig. Krankheiten der Hunde. I88I. p. 48.

2. JEss. Der Bacillus der Hundestaupe. (Febris catarrhalis epizootica Canines.) Cent. f. Bak. u. Parasitenk. Bd. 25. (I899) S. 54I.

3. Laossom. Ueber Geschichte and Kontagiosität der Staupe. Dorpat. 1892.

4. Nickolds. Dog distemper. Am. Let. Review. Igoo. p. ISo.

\section{INFECTIOUS CEREBRO-SPINAL MENINGITIS IN HORSES.}

$\$ 266$. Characterization. This is a disease, that seems to be infectious in its nature, exhibiting symptoms referable to a disturbance in the central nervous system. It is called epizoötic cerebro-spinal meningitis apparently because it often attacks a number of animals in the same locality. Although the literature contains numerous accounts of its seemingly contagious nature, an analysis of the facts fails to bring forth conclusive evidence that it is ever transmitted directly from one horse to another. In nearly if not all outbreaks, the animals affected have been subjected to like conditions of life. This disease is, at the present time, peculiar in that its cause is not known, that obvious tissue changes are usually absent, and that it has a very high mortality.

$\$ 267$. Etiology. The cause has been attributed to a great variety of conditions such as fermented food, forage laden with fungi or toxic moulds, various unsaritary conditions and possibly other ill-defined agencies. It seems to be 
true that in most outbreaks all of the animals that suffer have had at least some one thing in common either in surroundings, food or management. The bacteriological and other examinations which have been made to determine the cause have not resulted in finding a specific agent. Micrococci and various bacilli have been found associated with this disease. The writer has had an opportunity of making a careful examination of animals in two outbreaks. In one of them all inoculated media and histological examinations gave negative results, in the other pure cultures of a colon bacillus were obtained from the brain.

\$268. Symptoms. The mildest attacks are manifested by paresis or loss of perfect control over the limbs, loss of power over the tail, impairment of appetite and some difficulty in swallowing, together with areas of hyperemia and reddishbrown discoloration of the orbital and nasal mucosae. In other cases paralysis of one or more limbs may supervene but without marked fever or coma.

The more severe forms are ushered in by violent trembling, or by stupor, apathy and extreme muscular weakness or actual paralysis. In such cases the animal may stagger or fall. The inability to swallow is often a marked symptom, the saliva falling in strings from the lips. Another common phenomenon is the rigid contraction of the muscles of the neck, back and loins, the parts becoming tender to the touch and a more or less prominent opisthotonos setting in. Twitching of the muscles of the shoulders and flanks may be noticed. Trismus is sometimes seen. The breathing is usually rapid and catching and the temperature ranges from $104^{\circ}$ to $106^{\circ} \mathrm{F}$. The pulse may be accelerated and hard, soft and weak, or alternating. The eyes are usually violently congested, of a brownish or yellowish-red color and the eyeballs may be turned to one side. Paroxysms of delirium may set in when the animal will push against the wail or perform any of the disorderly movements following meningo-encephalitis. Sooner or later coma and paralysis supervene and death occurs in from five to forty-eight lours. In the most acute cases the animal falls and dies in convulsions. On an average the disease lasts 
from eight to fourteen days. In the more favorable cases, improvement may begin on the third or fourth day.

$\$ 269$. Morbid anatomy. Most writers report lesions of leptomeningitis, hyperemia of the brain and spinal cord witl extensive effusion into the ventricles and subarachnoid spaces. Petechire and parenchymatous degeneration of the solid organs of the body are also mentioned. In the cases examined by the writer there has been an absence of lesions in the nervous system and other organs that could be detected by a gross examination. In one case the brain, spinal cord and organs were studied histologically with like results. While there is undoubtedly structural changes somewhere in these organs, thus far they seem to have escaped detection. It is highly probable that up to the present time, cases of uncomplicated meningitis and possibly cerebritis have been confused with the disease in question. The entire subject must await the results of further investigation into both the etiology and morbid anatomy.

REFERENCES.

I. Favilde. So-called spinal meningitis. Am. Vet. Revieze. Vol. XVII. p. 9.

2. MARTIN. Cerebro-spinal meningitis. Ibid. Vol. XXI. p. 289.

3. SiEDAMGROTZKY AND SEHLEGEL. Epizoötic cerebro-spinal meningitis in the horse. (Abstract). Jour. Comp. Path. and Therapeutics. Vol. IX. p. 233 .

CORNSTALK DISEASE IN CATTLE.

\$270. Characterization. The name "cornstalk disease" has been given to a somewhat mysterious affection from which cattle sometimes suffer while feeding in cornstalk fields late in the fall and early winter. The meaning generally accepted and intended to be conveyed by this term is, that an animal or a number of animals, usually cattle, have died suddenly after feeding in a cornstalk field from + to io days. From a pathological point of view, therefore, the term is meaningless, but it has served admirably as a generic term to designate certain fatalities under a given condition which are not easily explained. 
The disease is very insidious, frequently causing the death of the animals before its presence is suspected. Cattle that act perfectly well at night are dead on the following morning. Usually all the animals in a herd that die of this affection perish in a single night or, at the longest, within a few days after the first death occurs. It is generally believed to be invariably fatal and its symptomatology has not been fully determined.

$\$ 271$. History. This affection of cattle has been known to exist for many years. The time and place of its origin, however, are not know1, but it seems to be peculiar to the United States and to have first appeared west of the Alleghany Mountains. We are told of its occurrence in the Mississippi Valley forty or more years ago. It made extensive ravages in $186+-65$ and again in 1868 . From an historical standpoint, no positive statements can be made concerning it prior to 1868 , when the first recorded investigations into its nature and cause were made. After that time the disease seems to have escaped the attention of investigators until 1889 , when Billings, of the Agricultural Experiment Station of Nebraska, published the results of his investigations.

In 1868, Gamgee was employed by the United States Department of Agriculture to investigate this disease. The "smut theory" of its etiology appears to have been the prevailing one at that time and consequently Gamgee's report deals almost exclusively with the effect of improperly prepared food, smuts and the like. Corn smut was unusually abundant in 1868 and he carried out experiments to test its etiological value with the conclusion "that smut is not a very active poison in combination with wholesome food."

In I889, Billings described the cornstalk disease as an " acute extraorganismal septicæmia, due to micro-organisus belonging to the class of ovoid-belted germs, to which variety of disease also belongs the swine plague, southern cattle plague, Wildseuche, hog cholera, and yellow fever in man." From the organs of cattle dead of the disease he reported to have invariably isolated a bacillus which he affirms to be its cause. He identified the bacillus which he found in the animal 
tissues with the one described by Burrill as the cause of a disease in cornstalks. In 1893, Dr. Theobald Smith identified the bacillus described by Professor Burrill as Bacillus cloace.

Billings also found pneumonia to be one of the lesions characteristic of this affection and in a subsequent bulletin he places great importance upon this lesion, although he adds very few additional observations to sustain the claim.

In I 890, a few animals from a shipload of American cattle landed at La Villette, France, died of pneumonia. They were examined very carefully by Nocard and other French veterinarians. From the diseased lung Nocard obtained a microorganism which corresponded very closely to the description of the bacillus of the cornstalk disease of cattle described by Billings in America. The publication of this fact gave rise to a temporary supposition that this American cornstalk disease might be a menace to the cattle of Europe and consequently initial steps were taken to require American cattle to be quarantined against it. The fact was subsequently determined that the bacillus isolated by Nocard belonged to the espticaemia hemorrhagica group of bacteria which is usually found to be associated with a form of bovine pneumonia occasionally met with in America, but not known to be contagious, and the matter was dropped.

A single experiment was made at Champaign, I11., in 1899. in which the etiological importance of corn smut was tested with negative results. A bacteriological examination of the organs from an animal that died in a cornstalk field, supposedly of this disease, was made with negative results by Professor Burrill in 1889 .

In I892, Moore investigated this disease and his report furnishes the information here given concerning the symptoms and morbid anatomy of this affection.

$\$$ 272. Geographical distribution. Geographically, this affection is restricted very largely to those sections of the United States where the farmers harvest their corn by picking the ears from the standing stalks, after which cattle are turned into the cornstalk fields. At present, therefore, it is limited in its distribution to the middle and northern portions of the 
Mississippi Valley. It is believed that there are exceptions to this and that rarely cattle die from its effects in the eastern part of the country.

\$273. Etiology. The definite cause of this disease has not been determined. It has been shown, however, that the former theories respecting salt, water and the bacillus of the Burrill disease of cornstalks are not substantiated. The hypothesis that the cause rests in the cornstalks themselves seems to be the most tenable. It is well known that cattle rarely if ever die from eating cormstalks in moderate quantities. However, the results of such investigations as have been made point as the cause to an intoxication from an excessive quantity of one or more elements existing in the cornstalks themselves.

S274. Symptoms. The insidious nature and rapid course of the disease usually results in the death of the animal before its presence is detected. In a few instances, however, the sick animals have been observed very carefully by their owners from whom all of our knowledge of the symptoms is obtained. One owner found a steer lying down and unable to rise. His head was extended, resting on the ground and moving continually from side to side; this was kept up for several hours, when he died. In the second case a steer was found to remain behind the other animals when they were driven to water about I $\mathrm{A}$. Ir. At $3 \mathrm{P}$. M. he was found in a dying condition.

Another man reported that he found his animals apparently well at 5 A. M. At 7 A. M. a heifer was found resting squarely upon her knees, the head extended and resting upon the ground. She was frothing at the mouth and groaning as if in great distress. She died in about two hours.

Another report states that the sick animals were dull, exhibited great weakness and walked with a peculiar jerky morement of the hind legs. The animals trembled as if suffering from a chill. In a short time they fell down and appeared to be in great distress, heads moving continually backward and forward. A fourth owner observed a sick animal trembling violently for about two hours before it died. 
275. Morbid anatomy. In twelve animals which died in cornstalk fields and were examined, post-mortem changes were more or less advanced in the greater number. A few were examined soon after death. The structural changes in the organs were slight. This explains the statement of many veterinarians that the organs of cattle dying of cornstalk disease are normal in appearance.

The only gross pathological changes observed were those of a hemorrhagic nature. The hemorrhages were mostly confined to the serous membranes and especially those of the heart. Ecchymoses were more or less numerous, however, beneath the pleura and under the capsule of the liver and of the spleen. The mucosa of the intestinal tract showed areas in which the capillaries were deeply injected. Occasionally. there were hemorrhages. In one case there were prononnced hemorrhagic areas in the heart muscle, aorta and pleura. In most cases the liver was pale in color and the acini presented deep reddish centers with pale peripheries. The inner and middle zones of the lobules were more densely congested than the peripheral ones. A layer several lobules in thickness immediately beneath the capsule was usually more engorged with blood than the deeper portions of the organ. In the more densely injected areas not only the blood spaces were filled, but the liver cells were also separated from each other by a space of variable width containing blood corpuscles. In some cases there were slight changes in the appearance of the cellular protoplasm.

In all of the animals examined, the hemorrhages were of recent origin and the blood normal in its microscopic appearance. There were no discolored areas indicative of old ecchymoses. The distribution and general character of the hemorrhages, which are the most conspicuous microscopic lesions observed, are very similar to those described in certain cases of vegetable poisoning, more particularly that of fern root.

Although certain symptoms described by the owners were referable to the nervous system, lesions were not discovered in the brain or spinal cord excepting in one case in which there was considerable hemorrhage beneath the pia mater. 
In one animal certain of the lymphatic glands exhibited more or less blood extravasation, particularly in the interlobular tissue.

276. Differential diagnosis. This disease is to be differentiated from sudden deaths due to various other causes. As a rule they are accidental although deaths directly due to engorgement, or to the consumption of too uuch corn, as might easily happen during the first days that the cattle are in the stalk field. The diagnosis of the cornstalk disease must, for the present, depend upon the hemorrhagic lesions and the period of feeding upon the stalks. Mayo has pointed out the fact that occasionally cattle die from eating cornstalks excessively rich in nitrate of potash.

Of the specific infectious diseases which might be confused with this affection acute anthrax, symptomatic anthrax and septicaemia hemorrhagica should be mentioned. Each of these affections can be determined from its specific nature as pointed out under the description of each of these affections.

\section{REFERENCES.}

I. Bilings. The cornstalk disease in cattle. Bulletins No. $7, \delta$. 9 and 10. Neb. Agric. Exper. Station. i886-88. p. 165.

2. BILLINGS, The corn fodder disease in cattle and other farm animals, with especial relation to contagious pleuro-pneumonia in American beeves in England. Bulletins . Vo.22 and 23. Univ. of Neb. Agric. Exper. Station. I892.

3. DE SCHWEINITZ. Cliemical examination of cornstalks presumably the cause of cornstalk disease in cattle. Bulletin No. IO, U.S. Bureau of Animal Industry. isg6.

4. Ganigen. Diseases of cattle in the United States. $U . S$. Department of Agriculture. 1869.

5. Mayo. Cattle poisoning by nitrate of potash. Bulletin No. 49 . Kansas Agric. Exper. Station. 1895.

6. MAYo. Cornstalk disease in cattle. Ibid. I8g6.

7. MOORE. An investigation into the nature, cause and means of preventing the cornstalk disease ('Toxæmia Maidis) of cattle. Bulletin No. Io. U. S. Bureau of Animal Industry. I896.

8. Nocard. Une Broncho-pneumonie infectieuse des Boeufs Americains. The cornstalk disease. Recucil de Médecine Vétérinaire. Tome VIII. (I89I). p. 424. 


\section{CHAPTER VIII}

a FEW Diseases CAUSED By ANIMAL PARASITES.

THE NODULAR DISEASE OF THE INTESTINES OF SHEEP.

$\$ 277$. Characterization. The intestinal, nodular disease of sheep is characterized by the presence of nodules in the upper part of the large intestine, although they are sometimes found in the walls of the ileum and jejunum, varying in size from that of a pin liead to that of a bean. They are caused by a nematode and because of their tubercle-like appearance the discase is often mistaken for tuberculosis. It is often called "knotty" viscera by butchers.

$\$ 278$. History. This disease has been observed in the eastern part of this country for a number of years. Just when it was introduced does not seem to be known. In I888-9 Dr. Cooper Curtice, then in the Bureau of Animal Industry, investigated this affection which up to that time was quite generally thought to be tuberculosis. The nodules had frequently been examined for tubercle bacilli, but with negative results. By means of serial sections and the microscopic examination of the fresh, small nodules, Curtice discorered the embryo of a nematode. The adult was found in the intestine in large numbers. It was readily referred to the genus Oesophagostoma and Curtice gave to this species the name of Columbianum because the worm was first found in the District of Columbia.

$\$ 279$. Geographical distribution. When first investigated, this disease seemed to be east of the Mississippi river. It was known to exist south of the Potomac river, but it had not been identified in the north. It is now known to be more or less prevalent in the niddle and New England states. 
\$280. Etiology. Prior to Curtice's discovery, this disease was generally considered to be tuberculosis. He showed, however, that it was caused by the embryo of Oesophagostoma Columbianum.

The life history of this parasite seems to be completely known to us during its development from the immature form found in the intestinal tumors to the adult stage: but there is a period from the moment when the eggs escape from the intestine with the excrement to the time when they are found in the intestinal tumors that remains obscure. After the embryo has returned into the alimentary canal it makes its way through the mucous coat of the intestine and becomes encysted there. Curtice was unable to learn how it passes through the mucous coat, as even on the youngest specimens no sign of armature is found. The very young forms found in cysts show little differentiation beyond what they could have attained in the shell. They are soon surrounded by a cyst which seems to belong to them and to have been created by then ; but whether this cyst is the remains of a molt has not been determined. Later in their history they become surrounded by the products of the inflammation they excite in the surrounding tissues and eventually break from the cyst and live in the cheesy mass of the tumor. In this stage of their growth the worms exhibit the intestine and oral cup and the indistinct unicellular glands. They then molt and show all these features in more distinct ontline. Having attained a length of from 3 to $+\mathrm{mm}$., or less than one sixth of an inch, they break from the tumors to begin tleir life in the intestine. In the latter they continue their growth and becoming sexually perfect, produce eggs which eventually go through the same cycle.

In developing, this worm molts at least three times-once in passing out of the stage in which it has no mouth or intestines, once during the development of these parts as we find them in the embryo and once while the worm changes from the embryonic form to the adult form.

$\$ 28 \mathrm{r}$. Morbid anatomy. The harm that these parasites do the sheep is directly dependent on their numbers and life 
history. Yearlings may show considerable infection, but it is usually in older sleep that the most abundant infection occurs. The disease is a seasonal one, in that it can be found in best development in the winter. The lambs begin to be infected

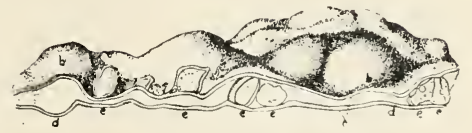

FIG. 69. Cross section of intestine containing nodules. b. mucous membrane, $c$. submucosa, d. muscular layers, e.e. section through cheesy nodules. (Curtice.)

in the summer and fall and from that time the tumors formed increase in size until early in the spring of the next year, when they gradually grow smaller but probably do not entirely disappear.

A study of the fresh tumors by compressing the smaller ones between two glasses and by dissecting larger specimens gives the following results. The small tumors, which are scarcely the size of a pinhead, are found in the submucous tissue. They appear like a sac filled with fluid and having a little globe floating within. By using a ligher magnifying power the little globe is seen to be a cyst with a worm inclosed. By careful dissection the cyst may be separated and finally the worm itself may be removed. In an older cyst there is a greenish, cheesy-like substance present.

When the little nodules become larger than a pinhead and entirely filled with the cheesy matter their structure does not materially change, but is more difficult to make out. It is at this stage that the worm escapes from the cyst and begins to wander within the capsule wlich its presence in the tissue has caused. On dissecting the large fresh tumors the worm is fornd in the mass of cheesy material, which is now quite abundant, filling the cavity and producing tumors as large as the end of one's finger. This cheesy material is usually hard, dry and brown at one end and soft, yellowish-green at the other. It is in this end that the worm is found. If some of the harder nodules are examined, it may be found that there is no greenish material in them and no worms. These hard 


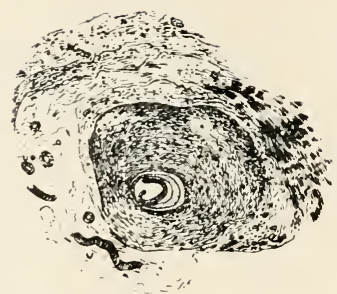

nodules may be of various sizes and are found at all times. From these the worm has either escaped or, in the case of the smaller nodules, the worms have died. The nodules are usually spherical, but the cheesy material may appear as a long mass or it may apparently fill what seems to have been a worm track. The last ap-

FIG. 7o. Section through a young pearance occurs most often in the worm nodule. Worm cavity with small intestines. From the muworm near the center. (Curtice). cous side these nodules present little if any color. The older ones may present a greenish-yellow appearance, especially if the mucous membrane over them is thin. In well advanced cases, when the nodules are numerous and large, many are found in which the mucous membrane over them is ruptured and the cheesy mass protrudes into the intestinal cavity. In these no worms have been found and hence the conclusion has been reached that they have escaped.

In lambs the little dots indicating the presence of the young nodules are very scattering, but in older sheep they may be very nuncrous. Between the stage in which a few are scattered here and there over the cecum and that in which the cecum has become a stiff tube with walls from one-fourth to one-half inch in thickness, there are all varieties. The nodules may extend from the cecum to the anus. They may also be abundant along the small intestine. The cheesy material which the worms produce has been found in the lymphatics, on the omentum and in the liver, but in these places it never seems to be sufficiently abundant to show that the parasite lived long.

Microscopic serial sections made from the alcoholic specimens show best the changes which have taken place in the surrounding tissue. The worm penetrates to the submucous tissue and irritates it. There is a special cyst for the rorm and a thickened adjoining portion of the tumor which is like a surrounding membrane. The adjoining cells rapidly increase 
in number so that the circulation from the inner cells is cut off and they die. Their degeneration gives rise to the clieesy mass. If the worm renained in the center of the mass the new growth would eventually cease, but the worm makes its way to the outside and at that point keeps up the irritation and new growth. This is shown by and accounts for the dried older parts of the larger nodules and the fresh yellowish-green adjoining parts. As soon as the worm escapes, the irritation ceases, the tumor shrinks and absorption of the mass begins. The irritation produced by the worm provides it with food and favorable surroundings for development. Often the worm dies from weakness or other cause and leaves behind those little liard incompletely-grown nodules which have been mentioned.

The disease is an insidious one, for not only is the rate of infection gradual, increasing slowly in amount from week to week, but the rate of development of the nodules is very slow, apparently requiring months. It is only when the disease is well advanced that its cumulative effects can be noticed.

$\leqslant$ 282. Differential diagnosis. The diagnosis of this parasitic disease can only be made from a post-mortem examination. In the living sheep there may be signs of general debility, bloodless lips and eyes, thin sides and flanks and dry wool. It may be that the flock is not in good condition ; and in severe cases the diarrhoea and emaciation may be excessive.

The only disease with which this can be readily confused is tuberculosis, which is very rare in sheep. A careful examination of the lesions will reveal the presence of the nematode which will decide the diagnosis. The fact that the lesions are usually restricted to the intestinal wall and that the nodules are sharply circumscribed from the normal tissue aid in the diagnosis from the gross specimen. It is important that this quite common disease should not be diagnosed as tuberculosis.

$\leqslant 283$. Prevention. For this disease there is no remedy except the removal and extermination of the adult worms. They are usually buried deeply in the mucous secretions of the intestinal mucosa to which they are attached. They may be found in considerable numbers in old sheep. The medi- 
cinal remedy must, therefore, be one which will remove them. It is possible that such a remedy may eventually be found, but at present it is not known. It is possible that some of the more reliable remedies advised for other intestinal parasites will do for these. The killing of the adults will of course lesson the number of eggs with which the sheep become infested. As the eggs pass to the ground the sheep may get them either while grazing or in their drinking water. The same care in changing pastures, in providing good drinking water and a plentiful supply of salt, should be observed as for the prevention of other parasites. Judicious fall and winter marketing of infested sleep will also tend to lesson the chances. of additional infection. If pastures are known to be permanently infested, they should not be used for sheep.

$\$$ 284. Nodular intestinal disease of cattle. Cattle suffer from a like parasitism, but from a different species of Oesopagostama. In the bovine species the nodules are, as in sheep, more conspicuous from the serous surface of the intestine than from the mucosa. They are important in that frequently they have been diagnosed as nodules of tuberculosis. As their structure is quite like those in sheep, the differential diagnosis can be easily made. If there is doubt, serial sections of a small nodule will usually reveal the presence of the worm. In cattle the oesophagostoma nodules do not appear to be of much if any importance in the animal economy.

\section{REFERENCES.}

CURTICE. The nodular disease of the intestines of sheep. Animal Parasites of Sheep. U. S. Bureau of Animal Industry. ISgo. p. 165.

\section{NODULAR TAENIASIS IN FOWLS.}

S285. Characterization. Nodular taeniasis in fowls is a disease of the intestines characterized by nodules varying in size from that of a pinhead to a small pea situated either beneath the mucosa, within the muscular walls or in the subserous tissue. It is caused by a small cestode. 
$\$$ 286. History. In $188 \mathrm{I}$, Piana described a disease of the intestines of fowls in Italy due to the presence of Tacnia bothrioplitis. His article deals more with the anatomy and classification of the cestode than with the character of the lesions it produced. However, he illustrates the nodules and heads of the tapeworms in the intestinal wall.

In 1894 , this disease was encountered in some fowls that were affected with diphtheria and that were under observation in the Burean of Animal Industry. The following year the disease was briefly described.

\$ 287. Geographical distribution. The extent of the distribution of this disease does not seem to be recorded. It had appeared in fowls from North Carolina, Virginia and the District of Columbia at the time it was described and since then a disease has been reported from the Eastern part of the State of New York and from Ohio which appears to be identical with it. As the inquiry into the cause of poultry diseases becomes more general, it is probable that this affection will be occasionally encountered, and unless its nature is recognized it may lead to an unwarranted destruction of property.

\$288. Etiology. As first pointed ont by Piana, the nodules are produced by a cell infiltration caused by the irritation of the head of a small cestode. Piana identified it as Tania bothrioplitis but Stiles places it as Davaina tetragona. There seems to be no other parasite of fowls capable of producing such lesions.

$\leqslant 289$. Symptoms. The only evidence of the disease, which is not characteristic of this affection, is a general emaciation.

290. Morbid anatomy. The lesions described for this disease are restricted to the intestinal wall. All of the fowls described, affected with this malady, were from one to three years old.

The nodules are invariably more numerous in the lowest third of the small intestine. They occasionally appeared, however, in small numbers in both the duodenum and colon. The larger and to all appearances older nodules are found in the ileuin near the creca. 
In the badly affected portion, the nodules give the appearance of closely set protuberances, varying in size from barely perceptible areas of elevation to bodies $+\mathrm{mm}$. in diameter. In some inst a nces

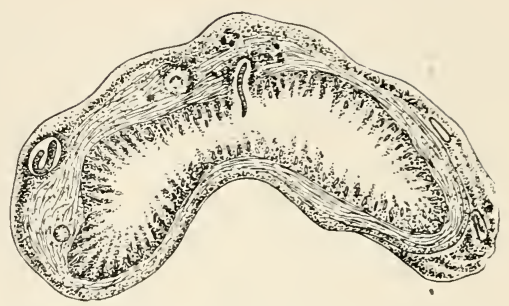

FIG. 7I. Cross sections of the affected intestine showing nodules, also attached worm.

they appear to overlap one another. When separated by a band of normal tissue, they are round or somewhat lenticular in form. In the latter case, the long diameter is usually transverse to the long axis of the intestine. The larger nodules are of a pale or dark yellowish color, while the smaller ones vary in shade from the more highly colored areas to the neutral gray of the normal serosa. To the touch they give the sensation that would be expected if the subserous and muscular coats were closely studded with small, oval, solid bodies. The mucosa presents similar elevations. Attached to the mucosa orer the nodules are a number of tapeworms. There are also in the nore advanced cases small ( 0.5 to I $\mathrm{mm}$.) areas over the larger nodules from which the mucosa has sloughed learing small ulcerated depressions.

The larger nodules contain a greenish-yellow, necrotic substance which appears, in the advanced stages, as a sequestrum with a roughened surface. On section it has a glistening, homogeneous appearance. Surrounding the necrotic substance is a thin layer of infiltrated tissue. The smaller nodules contain a more purulent-like substance and the smallest appear to the naked eye as areas of infiltration. Sections of the affected intestine show upon microscopic examination that the heads of the taperorms have penetrated the mucous membrane and are situated in different layers of the intestinal wall. They are frequently observed between the villi. As would be expected the heads are not readily detected in the masses of 
necrotic tissue contained in the larger nodules, but they are almost invariably found in the smaller ones. In a few sections the tapeworm has been traced through the mucosa to the nodule in the muscular tissue in which its liead appeared. In the earlier stage of the nodular development, there is a cell infiltration about the head of the worm. This process continues until the infiltrated tissue reaches a considerable size.

A larger form is commonly found in the intestinal contents. Although macroscopically they appear to be diferent, Dr. Stiles has found that they are presumably of the same species.

$\$$ 29I. Differential diagnosis. Tuberculosis is the only known disease for which this affection is liable to be mistaken and it is of much importance that the two diseases should not be confounded. The diagnosis has not in my experience been difficult, as in every case the attached tapeworms were readily detected upon a close examination of the intestinal contents, or of the mucous membrane of the infected portion of the intestine. However, the worms are quite small and could easily be overlooked in a hurried or cursory exanination. In case of doubt, if the affected intestine is opened, and the mucous surface
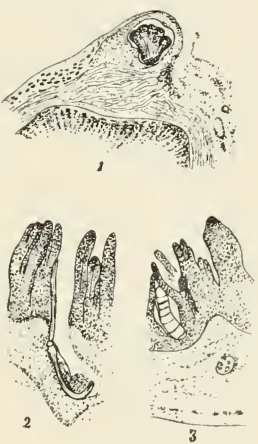

Fig. 72. (I) Cross section of a nodular containing necrotic cheesy tissue. (2) Cross section showing beginning of necrotic mass about head of a tape worm. (3) Section showing worm between villi and hooks within the muscle. washed carefully in a gentle stream of water, the small worms will be observed hanging to the mucous membrane. This discovery, in the absence of lesions in the liver or other organs, would warrant the diagnosis of the tapeworm disease. Although much is written concerning tuberculosis in fowls, the investigations of poultry diseases in America thus far show that it is not common among fowls in this country. 
1. MoOre. A nodular taeniasis in fowls. Circular No. 3, U.S. Bureau of Animal Industry. 1895.

2. Piana. Mem. della Accademia della Sc. Dell istitutodi Bologna. Ser. 4, 7, I1. (I880-8I ). p. 387 .

3. Stiles. Bulletin No. 12, U. S. Bureau of Animal Industry. I896. p. 49.

\section{TRICHINOSIS.}

$\$$ 292. Characterization. Trichinosis is the disease caused by the infesting of the muscles of the body with Trichinella spiralis. It affects man, swine and rats.

\$293. History. In I 835 , Paget, a medical student, found in a cadaver in a London dissecting room certain small worms which were described by Prof. Owen as Trichina spiralis. In the United States this parasite was observed in a negro in Charleston, S. C., by Chazal in $184 \mathrm{I}-2$ and in the muscles of a human cadaver in Boston by Bowditch in $18+2-3-4$. Stiles finds that about 900 cases have been reported in man in this country. In i 847 , Liedy found the same species of worm in the muscles of American swine. Herbst of Göttingen appears to have been the first to make an experimeutal inquiry into the life history of this parasite. In 1860 , Zenker showed it to be the cause of the disease which has since been named trichinosis, and which prior to his observations had been confounded with typhoid ferer and other maladies. The brilliant observations and experiments of Zenker, Leuckart, Virchow and others have given us detailed data concerning the life history and channels of infection of this parasite with the symptoms, duration of the disease, clinical details and the preventive measures to be adopted. During the years 1860 to 1866 , a number of outbreaks of trichinosis were experienced in certain European States more particularly Prussia and Saxony.

$\$ 294$. Description and life history of the parasite. Trichinella spiralis is a minute worm, the male a little over I $\mathrm{mm}$. long, the female about $3 \mathrm{~mm}$. in length. In the adult or sexual condition, it lives in the intestine of man, the pig and other mammals. Internal impregnation takes place, the eggs 
develop in the uterus of the female and the minute young to the number of at least a thousand are born alive. Soon after birth, the young worms migrate through the walls of the intestine and, following the course of the connective tissues, reach the voluntary muscles of the host, such as those of the limbs, back, tongue, muscles of the diaphragm and others. Each worm then penetrates the sarcolemma of the muscle fiber and coils itself up in the muscle substance. A spindle shaped cyst is formed around it, and the muscle undergoes more or less degeneration. This process gives rise to various morbid symptoms in the

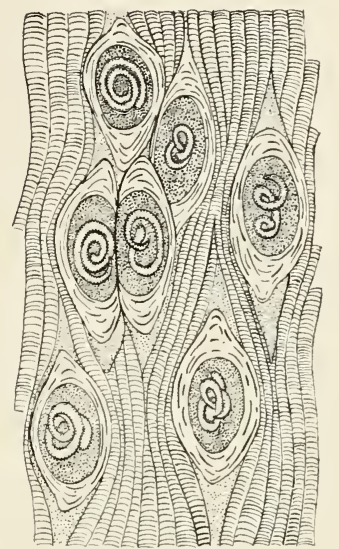

FIG. 73. Encapsulated Trihost but, after some months the chinella spiralis in striated muscysts become calcified and the cle. X 6o. (After Leuckart.) danger to the infested individual is over. The flesh of a "trichinized" human subject has been estimated to contain IOO, OOO, OOO and that of an infested pig 85,000 encysted worms to the ounce. In order that further development of the encysted and sexless trichinae should take place, it is necessary for the infested flesh of the host to be eaten by another animal in which the worm is capable of living, $e . g$., that of man by a pig or rat, or that of a pig by man. When this is done the cysts are dissolved by the digestive juices, the worms escape, develop reproductive organs and copulate, the young migrating into the muscles and producing the disease as before. The result of eating an ounce of "trichinized" pork, improperly cooked, might be the liberation in the intestine of perhaps 80,000 worms, and if half of these were females, each producing 1 , ooo embryos, some $40,000,000$ worms would shortly begin to migrate into the muscles and produce the various symptoms of "trichinosis." 
It will be noticed that in this case the parasite is able to exist in various hosts, and that both sexual and asexual stages are passed in the same host. The parasites are dispersed by the flesh of an infested animal being eaten uncooked by an uninfested one, either of the same or of a different species.

$\$$ 295. Manner of infection. The danger of human infection depends entirely upon the mode of preparation of the flesh. Thorough cooking, so that all parts of the meat reach or closely approach the boiling point, destroys the parasites. The frequency of the disease in different countries is referable, therefore, largely to the habits of the people in the preparation of pork. In North Germany where raw ham and wurst are eaten freely, the greatest number of cases have occurred. In South Germany, France and England cases are rare. In this country the greatest number of persons attacked have been those who eat raw or slightly cooked meat. Salting and smoking of the flesh as practiced in this country are believed to be sufficient to destroy the parasite. There may be exceptional cases. Carl Fraenkel states that it is very doubtful if any cases of trichinosis in Germany have been caused by American pork. It is stated that Germany has yet to show a single case of trichinosis due to pork of unquestioned American origin.

Swine become largely infested according to Stiles from eating rats. He made a careful investigation of this subject and found that the rats about country slanghter houses were infested to a large degree with trichinella. Hogs are fed in large numbers on the offal and they also frequently devour the rats.

$\$ 296$. Frequency. The dissecting room and post-mortem statistics show that from one-half to two per cent of all human bodies contain trichinella. The disease often occurs in epidemics, a large number of persons being infested from a single source.

In swine the frequency of this parasite varies, but from one to three per cent of the hogs whose muscles are examined microscopically contain trichinella. 
$\$ 297$. Symptoms. The ingestion of trichinous flesh is not necessarily followed by disease. When a limited number of the encysted larvae are eaten, only a few embryos pass to the muscles and may cause no symptoms. Well characterized cases in the human subject present two groups of symptoms, one referable to gastro-intestinal disturbances and the other to the general infesting of the muscles.

The following symptoms are described by Osler. In the course of a few days after eating the infested meat there are signs of gastro-intestinal disturbance, pain in the abdomen, loss of appetite, vomiting and sometimes diarrhoea. The preliminary symptoms, however, are by no means constant, and in some of the large epidemics, cases have been observed in which they have been absent. In other cases the gastrointestinal features have been marked from the outset, and the attack has resembled cholera nostras. Pains in different parts of the body, general debility and weakness have been noted in some of the epidemic cases.

The invasion symptoms develop between the seventh and tweifth day, sometimes not until the end of the second week. There is fever except in rery mild cases. Chills are not common. The thermometer may register 102 or $104^{\circ} \mathrm{F}$. and the fever is usually remittent or intermittent. The migration of the parasites in the muscles excites a more or less intense myositis, which is characterized by pain on pressure and movement and by swelling and tension of the muscles. The limbs are placed in the position in which the muscles are in the least tension. The involvment of the muscles of mastication and of the larynx may cause difficulty in chewing and swallowing. In severe cases the involvment of the diaphragm and intercostal muscles may lead to intense dyspnea which sometimes proves fatal. Oedema, a feature of great importance, may appear early in the face. Later it develops in the extremeties when the swelling and stiffness of the muscles are at their height. Profuse sweats, tingling and itching of the skin, and in some instances urticaria, have been described. The general nutrition is much disturbed and the patient becomes emaciated and often anæmic, particularly in protracted cases. The 
patellar tendon reflex may be absent. The patients are usually conscious, except in cases of very intense infestion in which delirium, dry tongue, and tremors give a picture similar to that of typhoid fever. In addition to the dyspnea present in most cases, there may be bronchitis, and in the fatal cases pneumonia or pleurisy. In some epidemics polyuria has been a common symptom. Albuminuria is frequent.

The intensity and duration of the symptoms depend entirely upon the grade of infestion. In mild cases recovery is complete in from ten to fourteen days. In severe forms convalescence is not established for from six to eight weeks, and it may be months before the patient recovers the muscular strength. One case in the Hedersleben epidemic was weak eight years after the attack.

Of 72 fatal cases in the Hedersleben epidemic the greatest mortality occurred in the fourth, fifth and sixth weeks; namely, 52 cases. Two died in the second week with severe choleraic symptoms. The mortality has ranged in different outbreaks from one or two per cent to thirty per cent.

The anatomical changes are said to be chiefly in the voluntary muscles. In the early stages the muscles look normal, but in the fourth or fifth week grayish-white areas appear in which the muscle fibers are extensively degenerated end in the neighborhood of the trichinella there is an acute interstitial myositosis. Cohnheim has described a fatty degeneration of the liver and enlargement of the mesenteric glands. At the time of death in the fourth or fifth week or later the adult trichinella are still found in the intestines.

The prognosis depends upon the quantity of infested meat which has been eaten and the number of trichinella which mature in the intestines. In children the outlook is more favorable. Early diarrhoea and moderately intense gastrointestinal symptoms are, as a rule, more favorable than constipation.

\$298. Diagnosis. The disease should always be suspected when a large birthday party or Fest among Germans is followed by cases of apparent typhoid fever. The parasites may be found in the remmants of the ham or sausages used on that 
occasion. The worms may be discovered in the stools. To find the worms, a portion of the feces should be spread on a glass plate or black background and examined with a low power lens. The trichinellae if present will appear as small, glistening, silvery threads. In doubtful cases the diagnosis may be made by the removal of a small fragment of muscle. A special harpoon has been devised for this purpose by means of which a small portion of the biceps or of the pectoral muscle may be readily removed. Under cocaine anaesthesia an incision may be made and a small fragment removed. Trichinosis may be mistaken for acute rheumatisn, particularly as the pains are severe on movement, but there is no special swelling of the joints. There is tenderness in the muscles both on pressure and on movement. The intensity of the gastro-intestinal symptoms in some cases has led to the diagnosis of cholera. Many of the former epidemics were doubtless diagnosed as typhoid fever. The pains in the muscles, swelling, oedema and shortness of breath are the most important diagnostic points.

It is practically impossible to diagnose this affection in living swine except by removing bits of infested muscle for microscopic examination.

S 299. Prevention. It is not definitely known how swine became diseased. It has been shown by Stiles that they are infested largely from rats about slaughter-houses, which are infested from eating portions of trichinous flesh of swine. It is very likely that hogs fed upon offal become infested. Swine should, as far as possible, be grain fed and not be allowed to eat offal. For the human subject the surest prevention is the thorough cooking of all pork and sausages.

\section{REFERENCES.}

WILLIAMS. The frequency of trichiniasis in the United States. Journal of Medical Research. Vol. VI. (1892). p. 64. 
APPENDIX.

\section{DISINFECTION.}

$\$ 300$. Disinfection. By disinfection is meant the destruction of infectious disease producing organisms. For this purpose, nature has provided very important agents such as sunlight and drying, but these are not available or sufficient to destroy all infecting bacteria in all infected places within the necessary time limits. To supplement these natural forces, a large number of chemical substances, possessed of germicidal powers, have been brought into service. If, however, the results of the test experiments with these different substances are reviewed, one is impressed with the discrepancies, if not contradictory conclusions, recorded concerning their value. In view of these facts the practitioner is often at a loss to know just what chemicals to use, or how to apply them under different conditions and for the destruction of different species of organisms. The failure resulting from the many efforts to disinfect stables, pens, kennels and yards has caused much skepticism concerning the efficiency of many reported disinfectants. In order to rightly understand the reason for the differences in results of the test experiments or the lack of uniformity in the application of the various disinfecting substances, it is well to take into account certain fundamental facts.

I. The bacteria used by different investigators to test the efficiency of certain substances have not been the same. The vital resistance of the various species is very different. The results obtained in testing disinfectants on the spirillum of Asiatic cholera or the bacterium of bubonic plagne give but little information relative to the value of the same disinfectants when used for the destruction of the bacteria of glanders, tuberculosis or hog cholera. Thus the difficulty in accepting 
the results of many of the older experiments is that test organisms were used which are very unlike those for which these disinfectants are $110 w$ wanted. The practical value of the more recent experiments is greater because they have more generally dealt with species of bacteria with which most of the work of disinfection has to do.

2. The power of resistance of the same species of bacterium varies greatly under different conditions. For instance, Bear found that a freshly inoculated culture of the bacterium of diphtheria was destroyed with $\mathrm{I}: 5000$ of nitrate of silver, but that a twenty-four hour culture required I: Iooo of the same agent to kill it in the same space of time. In some work done by Esmarch he made use of anthrax spores from seventeen different sources. They were destroyed by steam at $212^{\circ} \mathrm{F}$. in from one to twelve minutes and by a five per cent solution of carbolic acid in from two to forty-two days.

3. The medium in which the bacteria exists influences the results of the disinfectants. The bacterium of tuberculosis from an aqueous suspension dried upon threads may be promptly destroyed by mercuric chloride, but in fresh, purulent, tuberculous discharges it cannot be trusted to destroy thenn. Again Behring says that sporeless anthrax bacteria in water are killed by corrosive sublimate, I : 500, 000 : in bouillon, by I : 40,000; but in blood serum not with certainty with a solution of $I$ : 2000. Some disinfectants are influenced very much by the character of the material which contains the infectious organism, while other disinfectants are influenced to a comparatively slight degree. The experimental work which does not take the influence of the media upon the disinfectant into account is not of much practical value and failures are to be expected in the work of the disinfector who does not act in accordance with this fact.

4. The temperature under which the disinfecting agent acts influences very much the rapidity and the certainty of its action. Thus Heider found that anthrax spores that survived the action of a five per cent solution of carbolic acid thirty-six days at ordinary room temperature, were killed in from one to two hours at $13 \mathrm{I}^{\circ} \mathrm{F}$. Some investigators have failed to state 


\section{APPENDIX.}

\section{DISINFECTION.}

\$300. Disinfection. By disinfection is meant the destruction of infections disease producing organisms. For this purpose, nature has provided very important agents such as sunlight and drying, but these are not available or sufficient to destroy all infecting bacteria in all infected places within the necessary time limits. To supplement these natural forces, a large number of chemical substances, possessed of germicidal powers, have been brought into service. If, however, the results of the test experiments with these different substances are reviewed, one is impressed with the discrepancies, if not contradictory conclusions, recorded concerning their value. In riew of these facts the practitioner is often at a loss to know just what chemicals to use, or how to apply them under different conditions and for the destruction of different species of organisms. The failure resulting from the many efforts to disinfect stables, pens, kennels and yards has caused much skepticism concerning the efficiency of many reported disinfectants. In order to rightly understand the reason for the differences in results of the test experiments or the lack of uniformity in the application of the various disinfecting substances, it is well to take into account certain fundamental facts.

I. The bacteria used by different investigators to test the efficiency of certain substances have not been the same. The vital resistance of the various species is very different. The results obtained in testing disinfectants on the spirillum of Asiatic cholera or the bacterium of bubonic plague give but little information relative to the value of the same disinfectants when used for the destruction of the bacteria of glanders, tuberculosis or hog cholera. Thus the difficulty in accepting 
the results of many of the older experiments is that test organisms were used which are very unlike those for which these disinfectants are now wanted. The practical value of the more recent experiments is greater because they have more generally dealt with species of bacteria with which most of the work of disinfection has to do.

2. The power of resistance of the same species of bacterium varies greatly under different conditions. For instance, Bear found that a freshly inoculated culture of the bacterium of diphtheria was destroyed with I:5000 of nitrate of silver, but that a twenty-four hour culture required I: looo of the same agent to kill it in the same space of time. In some work done by Esmarch he made use of anthrax spores from seventeen different sources. They were destroyed by steam at $212^{\circ} \mathrm{F}$. in from one to twelve minutes and by a five per cent solution of carbolic acid in from two to forty-two days.

3. The medium in which the bacteria exists influences the results of the disinfectants. The bacterium of tuberculosis from an aqueous suspension dried upon threads may be promptly destroyed by mercuric chloride, but in fresh, purulent, tuberculous discharges it cannot be trusted to destroy them. Again Behring says that sporeless anthrax bacteria in water are killed by corrosive sublimate, I : 500,000; in bouillon, by I : 40,000; but in blood serum not with certainty with a solution of $1: 2000$. Some disinfectants are influenced very much by the character of the material which contains the infectious organism, while other disinfectants are influenced to a comparatively slight degree. The experimental work which does not take the influence of the media upon the disinfectant into account is not of much practical value and failures are to be expected in the work of the disinfector who does not act in accordance with this fact.

4. The temperature under which the disinfecting agent acts influences very much the rapidity and the certainty of its action. Thus Heider found that anthrax spores that survived the action of a five per cent solution of carbolic acid thirty-six days at ordinary room temperature, were killed in from one to two hours at $\mathrm{I}_{3} \mathrm{I}^{\circ} \mathrm{F}$. Some investigators have failed to state 
the temperature under which their disinfecting experiments were made.

5. In many of the results the inhibitory action of the agent in question has been mistaken for its germicidal action. After the bacteria have been subjected to the influence of a disinfectant for a given time, though not killed, their vegetating and pathogenic capabilities may be modified but still able, under favorable conditions, to return to their former vigor.

The rules and recommendations of the various cattle commissions and those having authority in methods for prevention of infectious diseases of animals do not very clearly define the procedures best adapted to the various places and conditions requiring disinfection. The many chenicals possessed of germicidal powers and the numerous commercial disinfectants, recommended largely from the results of certain definite tests, which in point of fact may be of no value in determining their efficiency for the conditions in question render further inquiry into the best methods for disinfecting after animal diseases a matter of much scientific interest and practical value.

s 3or. Conditions to be taken into account in practical disinfection. In the effort to destroy the nicroorganisms in such places as yards, stables, cattle cars and the like, it is necessary to consider before applying a disinfectant (1) the resistance of the particular organism to be destroyed, (2) the medium in which it exists, (3) the nature of the place containing the organisms to be destroyed and $(t)$ the clienical action of the material surrounding the microorganisms on the disinfectant itself. If the disinfection is for anthrax or Texas fever (cattle tick) a more powerful substance or solution must be employed than would be required in disinfecting for the bacteria of septicaemia hemorrhagica. If the infecting organisms are mixed with fecal matter, dirt or fodder, the problem is a different one than where they rest on a comparatively clear surface. It matters again whether the infecting organisms are in the soil (on surface), on a stable floor that is tight and hard or on one containing cracks of various sizes and made up of board, more or less shattered or decayed, thus furnishing deep recesses for the hiding away as it were of the specific organisms. 
In the disinfection of human dwellings the fumigation with formaldehyde has proven to be one of the cheapest and ordinarily the most efficient procedures, but it requires a tightly closed room. It is evident that such a method cannot be trusted for the disinfection of most barns, stalls or stables which are usually both large, compared with dwelling rooms, and what is of far more importance too open. For the disinfection from animal diseases the agents used must in most cases be applied in the form of a solution.

Jaeger's investigations brought out very clearly the necessity of adapting the disinfecting agent to the specific kind of infection to be destroyed. For instance, while brushing the surface witl a I : 3 milk of chloride of lime destroyed anthrax spores, it was untrustworthy as a disinfectant for the bacteria of tuberculosis and of glanders. For the destruction of the bacterium of tuberculosis he found carbolic acid and the other coal-tar phenols very efficient, especially wlien acidulated with hydrochloric acid. For this purpose he recommended especially Laplace's + per cent solution of crude carbolic acid with two per cent of hydrochloric acid. In the hands of Jaeger, the power to destroy anthrax spores with certainty has been shown only by solutions of carbolic acid and the thick chloride of lime mixture.

A thick milk of lime applied once with a brush, Jaeger found efficient in the destauction of the microorganisns of chicken cholera, hog cholera, erysipelas of swine, typhoid fever, glanders, anthrax bacillus without spores and staphylococcus pyogenes aureus.

Giaxa, in a similar line of work to that of Jaeger's, found that in the disinfection of walls even a fire per cent lime wash acting forty-eight hours failed to destroy anthrax spores, the bacterium of tuberculosis and the bacillus of tetanus.

A strong solution of the chloride of lime may be classed as one of the rapidly acting disinfectants for most bacteria, but Jaeger's report of its failure when applied to the infection of tuberculosis and glanders should be borne in mind. For the cleansing of cattle cars Gruber advises scrubbing them out with hot water or washing with a two per cent solution of soda 
at $50^{\circ} \mathrm{C}$., although this has no particular disinfecting power. If the cars are infected he sprays with a 5 to ro per cent solution of formaldehyde.

For disinfecting pens, stable floors and the like the following solutions have been recommended and their careful and intelligent use has shown them to be very efficacious.

I. Corrosive sublimate (mercuric chloride), $I$ ounce in 8 gallons of water (one-tenth of $I$ per cent). The water should be put into wooden tubs or barrels and the powdered sublimate added to it. The whole must be allowed to stand for twentyfour hours, so as to give the sublimate an opportunity to become entirely dissolved. Since this solution is poisonous, it should be kept covered up and well guarded. It may be applied with a broom or mop and should be used freely on all woodwork. Since it loses its virtue in proportion to the amount of dirt present, all manure and other dirt should be first removed before applying the disinfectant. The manure should be covered with lime.

2. Chlorinated lime. Five ounces of chloride of lime to a gallon of water (4 per cent). This should be applied in the same way as the corrosive sublimate.

3. A mixture of crude carbolic and sulphuric acids. The following disinfectant has been found to be very serviceable. It is not poisonous, but quite corrosive, and care should be taken to protect the eyes and hands from accidental splashing:

Crude carbolic acid $\ldots \ldots$ Gallon.
Crude sulphuric acid

These two substances should be mixed in tubs or glass vessels. The sulphuric acid is very slowly added to the carbolic acid. During the nixing a large amount of heat is dereloped. The disinfecting power of the mixture is heightened if the amount of heat is kept down by placing the tub or glass demijohn containing the carbolic acid in cold water while the sulphuric acid is being added. The resulting mixture is added to water in the ratio of $\mathrm{I}$ to 20 . One gallon of mixed acids will thus furnish 20 gallons of a strong disinfecting solution, having a slightly milky appearance. 
4. Ordinary slaked lime, though it does not possess the disinfecting power of the substances given above, is nevertheless very useful, and should be used more particularly on infected soil.

More recently the thorough sprinkling of the floors, walls, and ceilings with Formalin has been highly recommended.

In disinfecting stables and pens all litter which has accumulated should be 'removed before applying the disinfectant. As the litter itself is infected it should be disinfected as well as the stable, floor and walls. The most efficient method for this is fire. The practice of washing the floors and ceiling with water before applying the disinfectant has in most instances the disadvantage that the water carries the microorganisms to be destroyed into cracks and possibly through the floor where they will not be affected by the later application of the germicide. It is deemed safer, to simply use a dry cleaning in which the sweepings are burned and then apply the disinfectant in sufficient quantities to thoroughly saturate the surfaces including the adhering particles of dirt. The solutions available for stable disinfection are cheap enough to admit of this precaution.

It is often desirable to disinfect yards where infected animals have been kept. It is well in such cases to carefully scrape together and burn the litter after which the surface of the soil must be disinfected. For this the milk of lime or a a very liberal coating of slacked lime has been recommended. The burning of the surface as can be done by covering it with a laver of old straw and burning, is a more certain destroyer, especially if animal parasites are to be eliminated. The method of disinfecting with a flame as described by Ferbusch in 1896 and recently recommended by Stiles for disinfecting kennels, pens and yards offers possibilities not found in other means. The procedure consists of a "Cyclone burner" attached to the end of a long iron tube with a wooden shield. This is connected with a short piece of hose of the kind made for the delivering of oil attached to a spray pump. A tank of suitable size is taken and paraffin gas oil is used: The spray of oil is ignited giving a very hot and effective flame which is run over 
the surface to be disinfected. The flame destroys all living things on the surface. This method seems to be applicable to the disinfection of floors and stables as well as yards, when the conditions are permissible and when the flame can be applied with the necessary care. Experiments are being made to determine more definitely the extent to which this method can be applied with safety.

\section{REFERENCES.}

I. RIDEAL. Disinfectants and disinfection. London. IS95.

2. STILES. The disinfection of kennels, Pens and yards by fire. Bulletin No. 35, U. S. Bureau of Aninal Industry. I902. p. I5.

3. Young. Notes on disinfectants and disinfection. Reprinted from the roth Report of the State Board of Health of Maine. I898. (Contains full bibliography on disinfectants and results of experiments.) 


\section{INDEX.}

Actinomycosis, 204.

Characterization, 204.

Differential diagnosis, 2 I5.

Etiology, 206.

Geographical distribution, 205.

History, 204.

Infection, 207.

Morbid anatomy, 209.

Sanitary considerations, 216.

specific treatment, 216.

Symptoms, 20 S.

Anoeba dysenteriae, 256 .

A moeba meleagridis, 254 .

Animal inoculation, 123, 151 .

Animal parasites, diseases caused by, 355 .

Anthrax, Si.

Animals attacked, $S_{5}$.

Channels of infection, $\mathrm{S}_{5}$.

Characterization, si.

Differential diagnosis, 93.

Disposition of a nimals dead of anthrax, 97.

Eitiology, 83 .

Geographical distribution, 82 .

History, $\mathrm{S}_{2}$.

Morbid anatony, go.

Pasteur's anthrax vaccine, 95.

Protective inoculation, 94.

Srmptonis, 87 .

Toussaint's antlirax vaccine, 96 . Law's modification of, 96 .

A poplectiform septicremia in chickens, 44.

Characterization, 44 .

Differential diagnosis, 46 .

Etiology, 44.

Geographical distribution, 44.

History, 44

Morbid anatomy, 45 .

Prevention, 46.

Srmptoms, 45 .

A specific infectious disease, 3 .

Aspergillus fumigatus, 230.

Asthenia in fowls and pigeons, 167.

Characterization, 167 .

Ettiology, 167 .

History, 167.

Morbid anatomy, 168.

Symptoms, 168.
Bacillus cholerce suis, I7 r, I8I.

Brief description of, I7I.

Bacillus cloaca, 35 r.

Eacillus tetani, I86.

Bacteria causing wound infection, 6 .

Bacterium anthracts, $\mathrm{S}_{3}$.

Bacterium asthenia, 167.

Bacterium of swine plague $18 \mathrm{r}$.

Bacterium septiccmiae hemorrhagicae, 4 s, 70 .

Bacterium tuberculosis, 131 .

Cultivation of, 131 .

Black head (See infectious enterohepatitis).

Boöphilis bovis, $24 \mathrm{I}$.

Botryomycosis, 7 .

Cerebro-spinal meningitis (See infectious cerebro spinal meningitis).

Channels of infection, 4 .

Through abrasions of skin, 5 . " agency of insects, 5 . " digestive tract, 4 .

“. generative organs, 5 .

“ respiratory tract, 5 .

“ transmission from parent to foetus, 5 .

Contagious pleuro-pneumonia in cattle, 290.

Characterization, 290.

Differential diagnosis, 299.

Eradication, 301.

Etiology, 293.

History, 290.

In the [. S., 29 I.

Iorbid anatomy, 296.

Preventive inoculation, $30 \mathrm{r}$.

Srmptoms, 294.

Cornstalk disease, 349 .

Characterization, 349 .

Differential diagnosis, 354.

Etiology, 352

Geographical distridution, $35^{\text {I }}$.

Historv, 350 .

Morbid anatomy, 353 .

Srmptoms, 352

Davainae tetragona, 36r.

Diarrhoea in calves (See white scours)

Diphtheria in fow 1 s, 325 . 
Characterization, 325 .

Etiology, 326.

History, 326 .

Morbid a natomy, 328 .

Prevention, 332.

Relation to human diphtheria, 330.

Symptonis, 327 .

Disinfectants, 374 .

Disinfection, 370 .

Dog distemper, 340.

Characterization, 340 .

Differential diagnosis, 346 .

Etiology, 340.

Geographical distribution, 340 .

History, 340 .

Morbid anatomy, 344 .

Prevention, 347.

Symptoms, $34 \mathrm{I}$.

Dourine, 272, 274.

Characterization, 274.

Etiology, 274 .

History, 274 .

Morbid anatomy, 278.

Prevention, 281 .

Symptoms, 276 .

Equine contageous pleuro-pneumonia, 37 .

Characterization, 37 .

Differential diagnosis, 44.

Etiology, 38

Geographical distribution, 37.

History, 37 .

Morbid anatomy, 4I.

Symptoms, 40.

Etiologr, 1.

Farcy (See glanders).

Fistulous withers, 15.

Foot and mouth disease, 302.

Characterization, $3 \mathrm{O} 2$.

Differential diagnosis, 306 .

Etiology, 303 .

Geographical distribution, $3 \mathrm{O}_{3}$.

History, 303.

Morbid anatomy, 305.

Prevention, 306.

Syniptoms, 303.

Fowl cholera, 77

Characterization, 77

Differential diagnosis, So.

Eitiology, 78 .

Geographical distribntion, 78 .

History, 77 .

Morbid anatomy, 79 .

Prevention, 81 .

Symptoms, $7 \mathrm{~S}$.
Glanders, I I 3.

Characterization, $\mathrm{I}_{3} 3$.

Differential diagnosis, 22.

Etiology, I15.

Geographical distribution, 114 .

Glanders in man, I21.

History, 113 .

Morbid anatomy, 117 .

Symptoms, II5.

Hemorrhagic septicaemia in cattle, 68 .

Characterization, 68 .

Differential diagnosis, 76 .

Etiology, 7o.

Geographical distribution, 70 .

History, 68 .

Morbid anatomy, 72 .

Prevention, $; 6$.

Symptonis, 7I.

Hog cholera, 169.

Characterization, 169.

Differential diagnosis, I So.

Etiology, 171 .

Geographical distribution, I7I.

History, 169.

Morbid anatomy, 175 .

Prevention, Is'.

Specific treatment, I $\delta_{4}$.

Symptoms, I 74 .

Infection, 2.

Infectious cerebro-spinal meningitis, 347 .

Characterization, 347 .

Etiology, 347 .

Morbid anatomy, 349 .

Symptoms, 348 .

Infections entero-hepatitis, 252.

Characterization, 252.

Differential diagnosis, 264.

Etiology, 254.

Geographical distribution, 253.

History, 253.

Morbid anatomy, 257.

Post-mortem notes, 263.

Prevention, 264.

Srmptons, 256

Infectious leukaenia in fowls, 98 .

Characterization, 98 .

Differential diagnosis, I05.

Etiology, 99

Geographical distribution, 9 S.

Historv, 98.

Morbid anatomy, IoI.

Symptoil1s, 99.

Infectious mastitis, I 5 .

Bacteria of, IS. 
Infectious pneumonia in swine (See swine plague).

Influenza, 334

Characterization, 334 .

Differential diagnosis, 339.

Etiology, 33.5 .

Geographical distribution, 335 .

History, 335.

Morbid anatomy, 338 .

Symptoms, 336.

Leeches, 218 .

Characterization, 218.

Etiology, 22 I.

Geographical distribution, 22 I.

History, 220.

Morbid anatomy, 222.

Treatment, 228 .

Mal de caderas, 273.

Mallein, 123.

Micrococcus lanceolatus, 48 .

Migula's classification of bacteria, 25.

Nagana, 272

Nodular disease in cattle, 360.

Nodular disease of sheep. 355 .

Characterization, 355 .

Differential diagnosis, 259.

E,tiologv, 356 .

Geographical distribution, 355 .

History, 355 .

Morbid anatonyy, 356 .

Prevention, 359

Normal temperature of cattle, I56. Effect of drinking wateron, I 57.

Esophagostoma Columbianum, 356.

Omphalophlebitis, 8 .

Ovine caseous lymph adenitis, I6I. Characterization, $16 \mathrm{I}$.

Differential diagnosis, 166.

Etiology, I62.

Geographica1 distribution, 162 . Historv, I6I.

Norbid anatomy, 163 .

Symptoms, I62.

Pleuro-pneumonia (See contagious pleuro-pneumonia.

Pneuniomycosis, 228.

Morbid anatomy, 231.

Species of fungi, 228.

Poll evil, I5.

Practical disinfection, 372.

Pyroplasma bigeminum, 23 S.

Pyrosoma bigeminum, 238.

Rabies, 306

Differential diagnosis, 320 .
Etiology, 308 .

Geographical distribution, 307.

Histury, 307.

Method of invasion, 309.

Morbid ariatomy, 3 I5.

Period of incubation, 310

Prevention, 324.

Symptoms, 31 2.

Treatment, 324 .

Ray fungus, 206 .

Rinderpest, 282.

Characterization, 282 .

Differential diagnosis, 287.

Etiology, $28_{3}$.

Geographical distribution, $2 \mathrm{~S}_{3}$.

History, 282.

Morbid anatony, 285 .

Prevention, 287.

Septicamia, 3 .

Specific infectious disease, 19.

Cause of variation in, 22.

Differential characters of, 20 .

Grouping of, 22

Strangles, 33 .

Characterization, 33 .

Differential diagnosis, 36 .

Etiology, 34

Geographical distribution, 33 .

History, 33 .

Morbid anatomy, 35 .

Symptoins, 34 .

Streptococci, 27

Classification of, 29.

Diseases due to, 27.

Distribution in nature, 30 .

General discussion of, 27.

Surra, 265.

Characterization, 265 .

Differential diagnosis, 272.

Etiology, 266.

Geographical distribution, 265.

Historv, 265.

Morbid anatomy, 268.

Prevention, 272.

Report of Smith and Kinyoun, 269.

Symptoms, 267.

Swine erysipelas, 107 .

Characterization, 107.

Differential diagnosis, I I I.

Etiology, ros.

Geographical distribution, 107.

Morbid anatomy, Iog.

Preventive inoculation, II I.

Symptonis, Io8.

Swine plague, 47 . 
Autopsy notes, 59 .

Bacterium of, 50 .

Characterization, 47 .

Differential diagnosis, 61 .

Etiology, 48 .

Geographical distribution, 48 .

History, 47.

In rabbits, 63 .

Modification of in rabbits, 64 .

Morbid anatomy, 52 .

Prevention, 62.

Specific treatment, 63 .

Symptons, 52

Symptomatic anthrax, 195.

Characterization, I95.

Differential diagnosis, 200.

Etiology, 196

Geographical distribution, I 96.

History, 195.

Morbid anatomy, 198.

Prevention, 201.

Preventive inoculation, $2 \mathrm{O} 2$.

Symptons, 197.

Tenice bothrioplitis(See Davainæ).

Tieniasis in fowls, 360 .

Characterization, 360 .

Differential diagnosis, 363 .

Etiology, 36r.

Geographical distribution, 361.

History, 36r.

Morbid anatomy, 36r.

Symptoins, 36r.

Tetanus, IS5.

Characterization, 185 ,

Differential diagnosis, 192.

Etiology, 186.

Geographical distribution, 185 .

History, $\mathrm{IS}_{5}$.

Mode of infection, I 87 .

Morbid anatomy, I9o.

Prevention, 193.

Symptons, ISS.

Tetanus antitoxin, 193.

Texas fever, 236 .

Characterization, 236.

Differential diagnosis, 249.

Etiology, $23 \mathrm{~S}$.

Geographical distribution, 237.
History, 236.

Immunizing susceptible cattle, 249.

Infection, 240.

Morbid anatomy, 245 .

Prevention, 249.

Symptoms, 244.

Toxaemia, 3 .

Trichinosis, 364 .

Characterization, 364 .

Description of 'T. spirallis, 364 .

Diagnosis, 368.

Frequency, 366 .

History, 364 .

Manner of infection, 366.

IIorbid anatomy, 368 .

Prevention, 369 .

Symptoms, 367 .

Trypanosoma Brucei, 272.

" equinum, 273.

" equiperdum, 272.

“" Evansi, 266.

“L Lewis, 273.

Trypanosomiasis, 272 .

Tse tsefl y disease, 272 .

Tuberculin, $15 \mathrm{I}, 235$.

Application of, 154.

Preparation of, I52.

Tuberculosis, 127.

Characterization, 127.

Differential diagnosis, I 5 O.

Etiology, I3I.

Extent of in cattle and swine, 129.

Geographical distribution, I30.

History, 127.

Local and generalized, I44.

Morbid anatomy, 137 .

Prevention, I5S.

Symptoms, I 33 .

Tuberculosis in swine, I44.

Svmptoms, 147.

Morbid anatomy, 148 .

White scours in calves, Io.

Nocard's treatment of, 12.

Wound infection, 3,6 .

Bacteria causing, 6 .

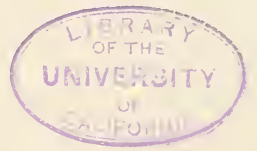





THIS BOOK IS DUE ON THE LAST DATE STAMPED BELOW

AN INITIAL FINE OF 25 CENTS WILL BE ASSESSED FOR FAILURE TO RETURN THIS BOOK ON THE DATE DUE. THE PENALTY WILL INCREASE TO 50 CENTS ON THE FOURTH DAY AND TO $\$ 1.00$ ON THE SEVENTH DAY OVERDUE.

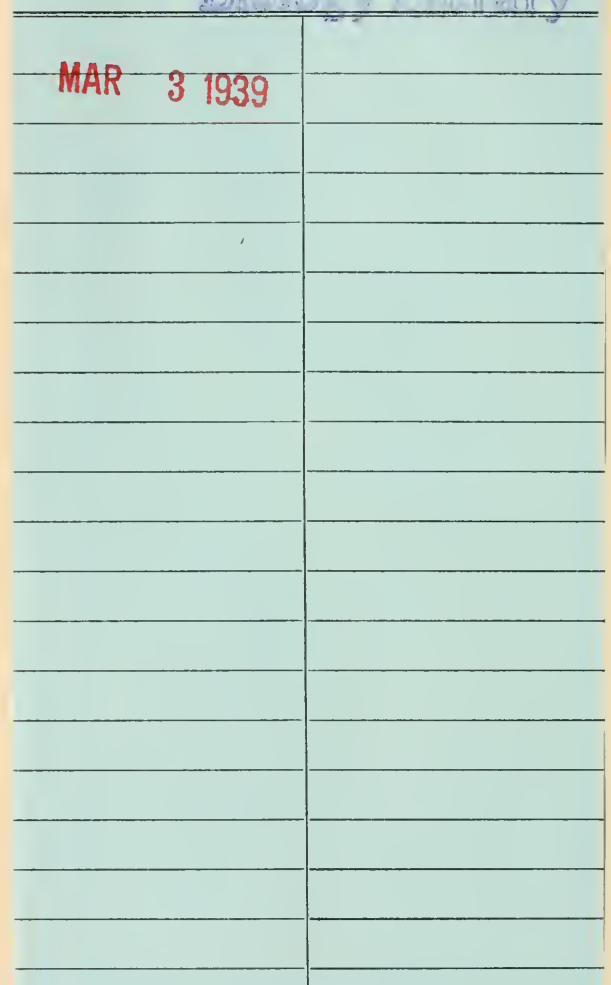

LD 21-95m-7,'37 


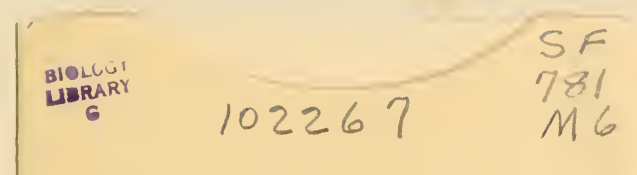

THE UNIVERSITY OF CALIFORNIA LIBRARY 
